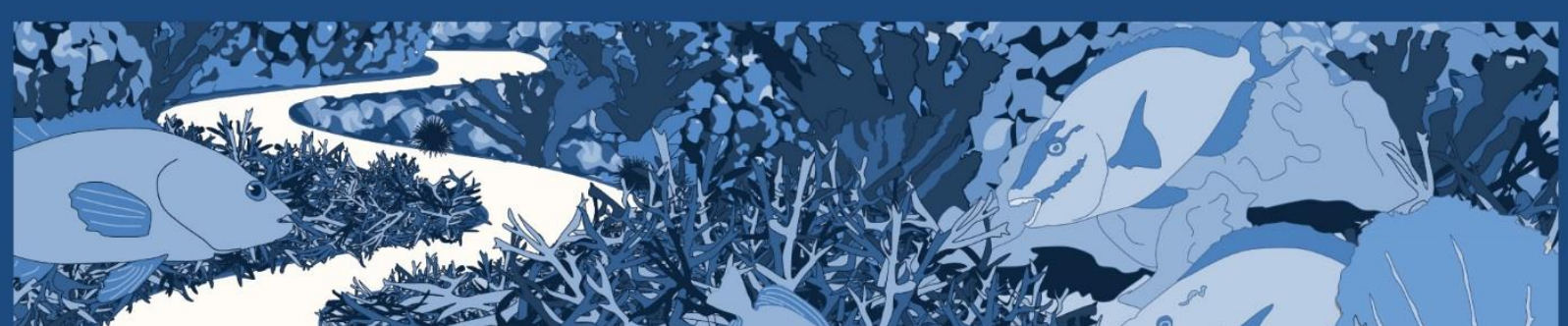
$12,1,0$

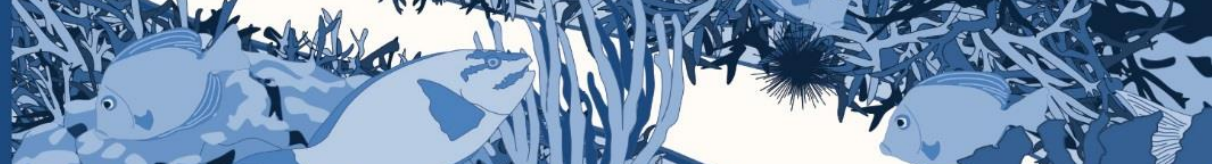

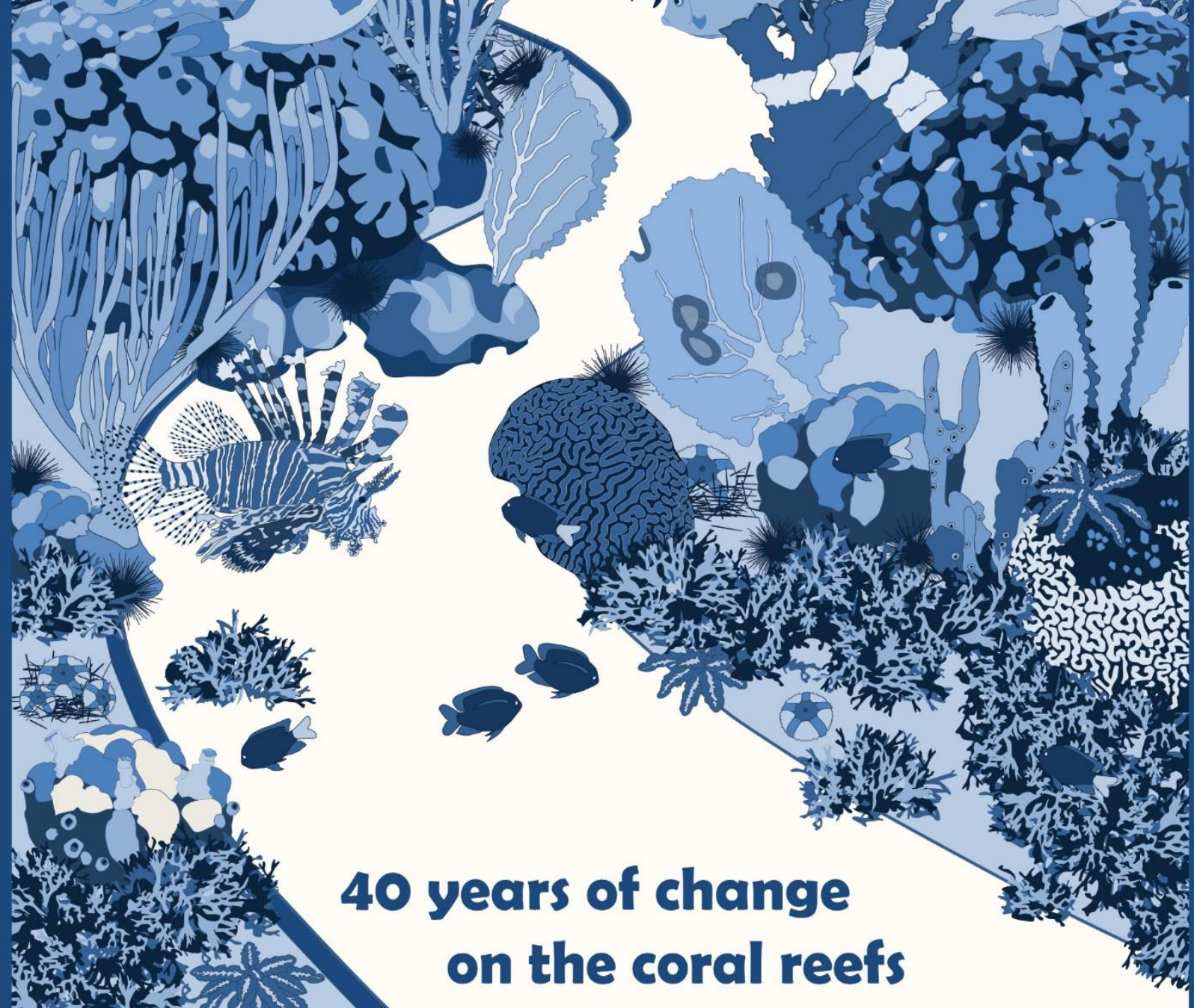

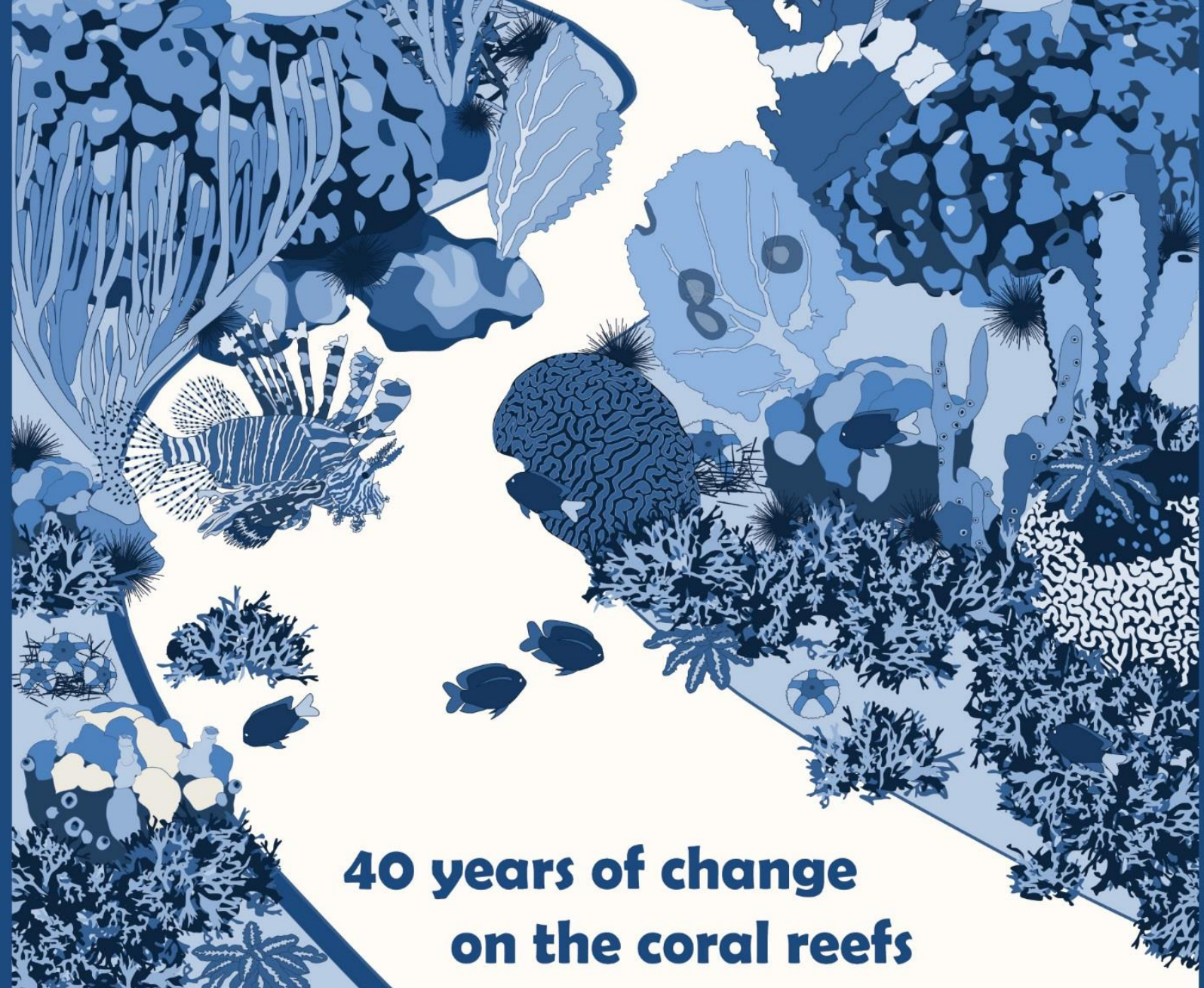

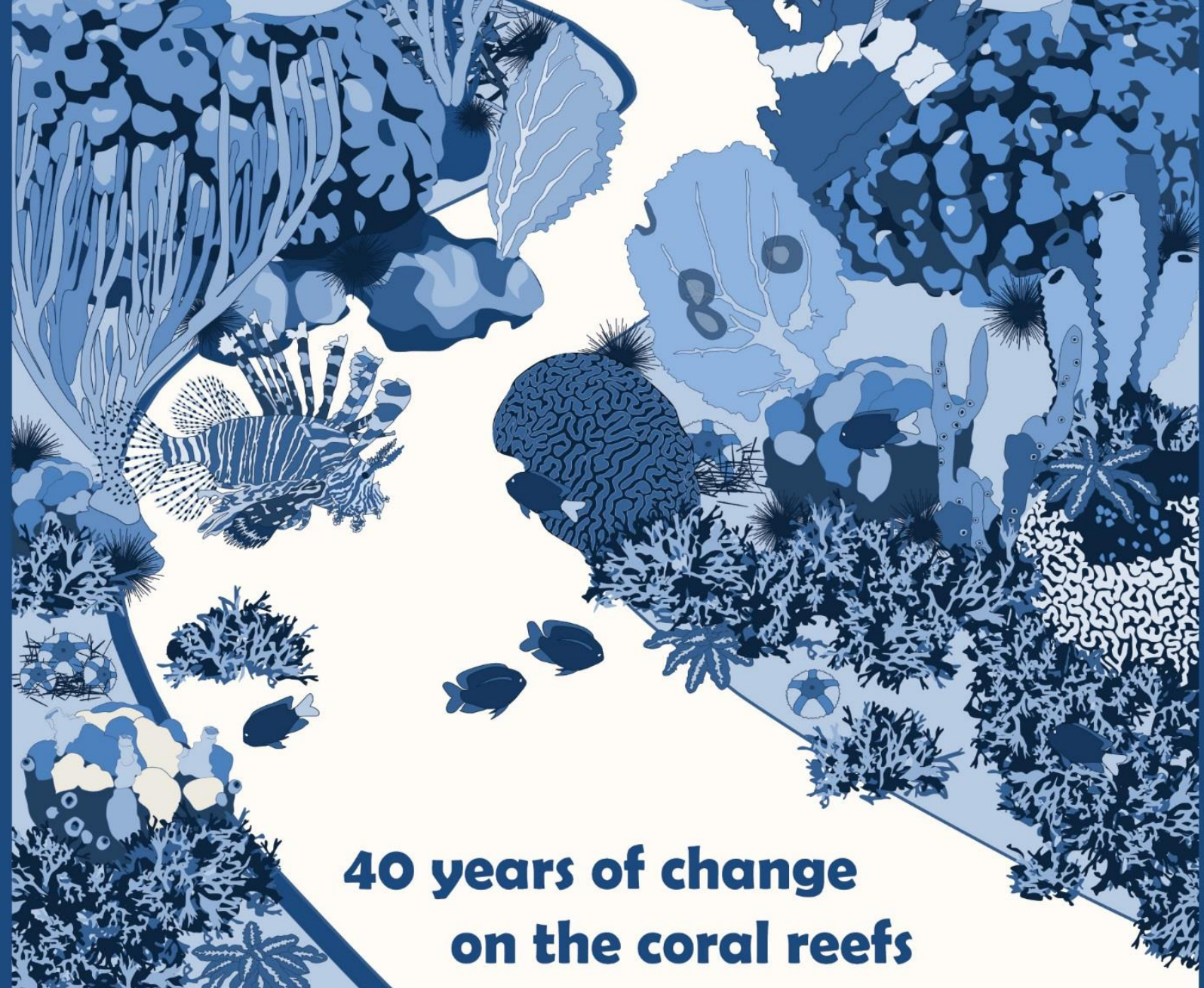

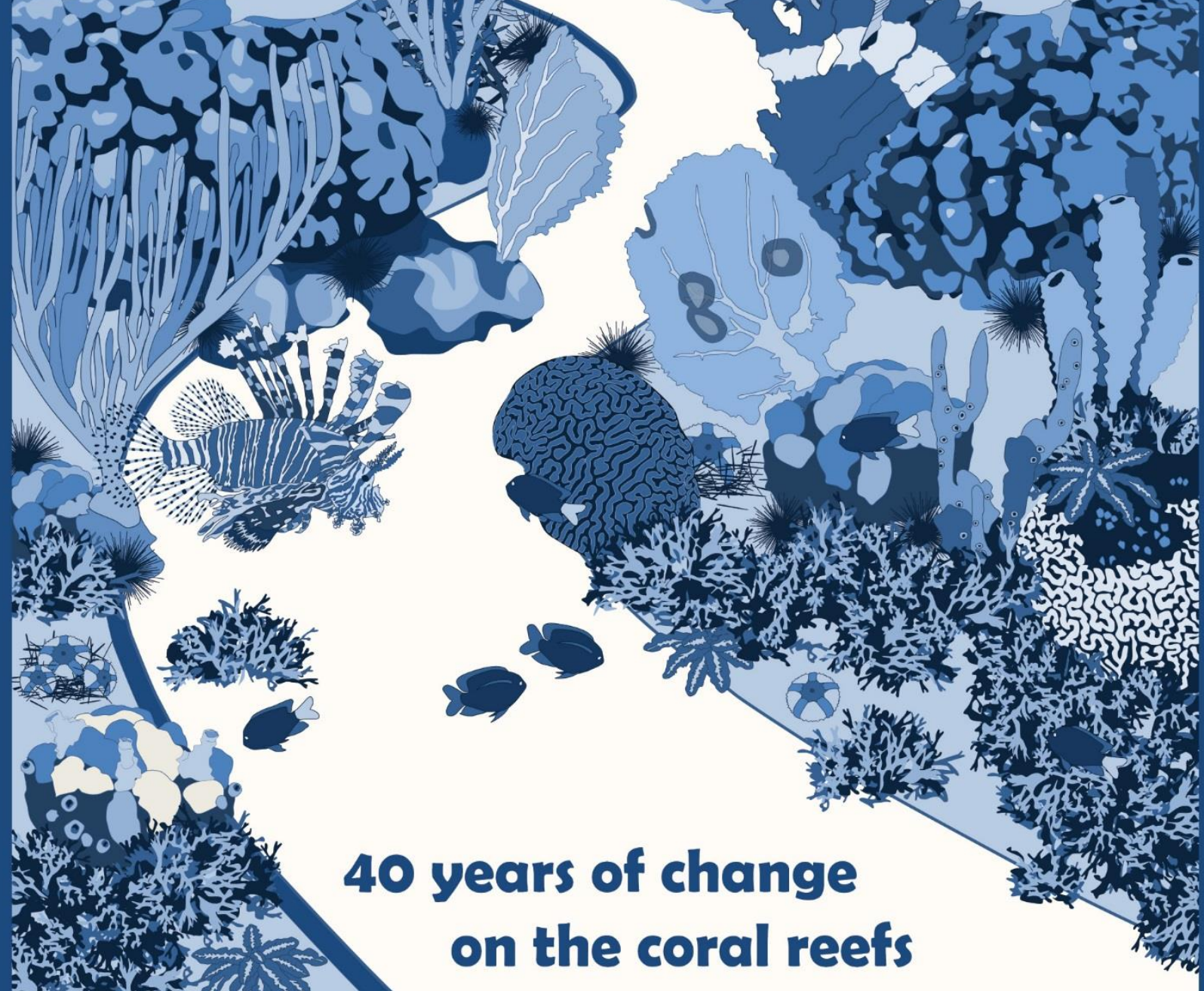

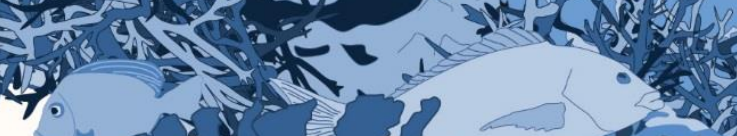
3 .

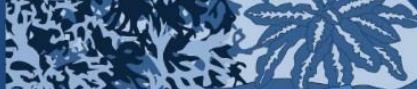
y. of Curaçao and Bonaire 


\section{0 years of change on the coral reefs of Curaçao and Bonaire}

Didier (Desiderius) Marinus de Bakker 


\section{Thesis committee}

\section{Promotors}

Prof. Dr H. J. Lindeboom

Professor of Marine Ecology

Wageningen University \& Research

Dr E.H. Meesters

Tropical Marine Ecologist, Wageningen Marine Research

Wageningen University \& Research

Co-promotor

Dr F.C. van Duyl

Senior Scientist, Microbiology \& Biogeochemistry

Royal Netherlands Institute for Sea Research, Texel \& Utrecht University

\section{Other members}

Prof. Dr R.H. Wijffels, Wageningen University \& Research

Prof. Dr C.J.M. Philippart, Royal Netherlands Institute for Sea Research, Texel | UU

Dr L.E. Becking, Wageningen University \& Research

Dr M.J.A. Vermeij, University of Amsterdam | CARMABI Foundation, Curaçao

This research was conducted under the auspices of the Graduate School for Socio-Economic and Natural Sciences of the Environment (SENSE). 


\title{
40 years of change on the coral reefs of Curaçao and Bonaire
}

\author{
Didier (Desiderius) Marinus de Bakker
}

Thesis

submitted in fulfilment of the requirements for the degree of doctor at Wageningen University

by the authority of Rector Magnificus,

Prof. Dr. A.P.J. Mol,

in the presence of the

Thesis Committee appointed by the Academic Board

to be defended in public

on Wednesday the $17^{\text {th }}$ of April 2019

at 1:30 p.m. in the Aula. 
Didier M. de Bakker

40 years of change on the coral reefs of Curaçao and Bonaire, 192 pages.

$\mathrm{PhD}$ thesis, Wageningen University, Wageningen, the Netherlands (2019)

With references, with summary in English

ISBN 978-94-6343-404-1

DOI https://doi.org/10.18174/468014 
Voor mijn lieve ouders en zussen

Deep beneath the rolling waves in labyrinths of coral caves an echo of a distant time comes willowing across the sand

Echoes, Pink Floyd 


\section{Table of content}

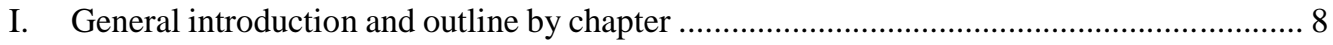

II. 40 Years of benthic community change on the Caribbean reefs of Curaçao and Bonaire: the

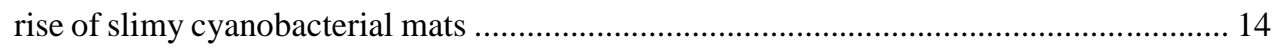

III. Long-term shifts in coral communities on shallow to deep reef slopes of Curaçao and Bonaire: are there any winners?

IV. Quantification of chemical and mechanical bioerosion rates of six Caribbean excavating sponge species found on the coral reefs of Curaçao 66

V. Spatial variation in coral reef accretion potential poses significant concern for low-lying tropical coastlines

VI. Towards stabilization of the decline in coral cover and fish biomass on the shallow reefs of

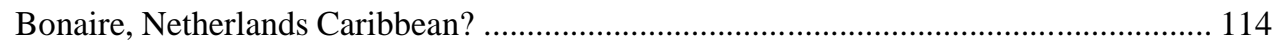

VII. General discussion: is there hope for Caribbean coral reefs? ...................................... 148

\section{Epilogue:}

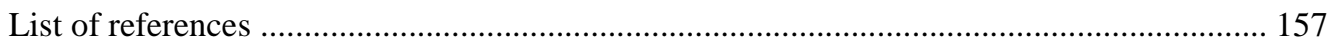

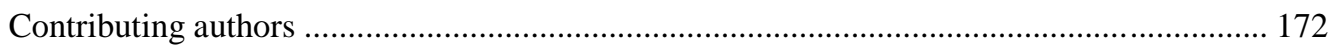

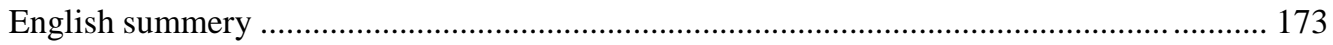

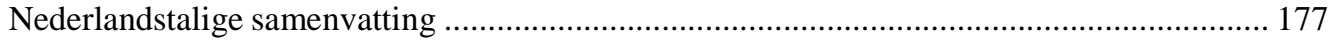

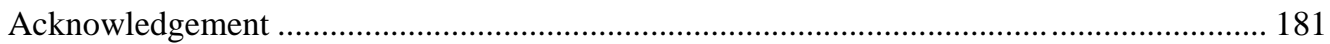

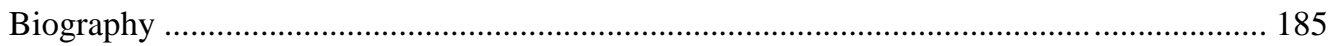

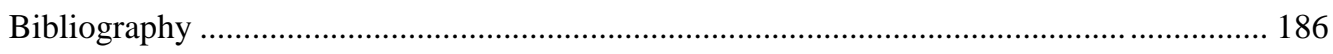

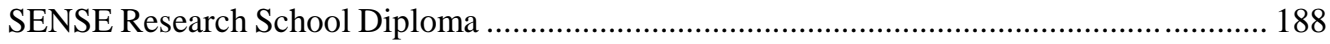




\section{Chapter I}

\section{General introduction and outline of this thesis}

Didier M. de Bakker

"I can mention many moments that were unforgettable and revelatory. But the most single revelatory three minutes was the first time I put on scuba gear and dived on a coral reef."

Sir David Attenborough

\section{Coral reef ecosystems}

Tropical coral reefs are among the most productive and biologically diverse ecosystems found on earth (Odum and Odum 1955; Connell 1978; Moberg and Rönnbäck 2003). Although these reefs only cover $0.1-0.5 \%$ of the ocean floor they provide a home to almost one third of the marine fish species and other marine biota (Mcallister 1991; Spalding and Grenfell 1997; Spalding et al. 2001). Like rainforests, their terrestrial equivalent, the three-dimensional habitat complexity underpins the biological success of coral reef systems (Connell 1978; Grigg et al. 1984; Reaka-Kudla 1997). This structural framework is primarily provided through the precipitation of vast quantities of calcium carbonate by scleractinian corals (Goreau 1959b; Goreau and Goreau 1959; Smith and Kinsey 1976). Basic growth of coral skeleton forms the fundament of the reef and facilitates complex ecosystem functioning and niche partitioning to harbour an exceptional heterogeneity of associated biota (Connell 1978; Graham and Nash 2012; Kennedy et al. 2013; Newman et al. 2015). Ancillary to the inexpressible biological value, millions of people worldwide rely in some way on the services provided by coral reefs, most notably for nourishment, but also for services associated with tourism and coastal protection (Costanza et al. 1997; Moberg and Folke 1999; Moberg and Rönnbäck 2003). By increasing frictional dissipation of wave energy, the complex physical structure created by corals protects coastal shorelines from erosion. This has allowed humans to settle and develop coastal areas throughout the tropics. Yet, coral reefs are at present ubiquitously under pressure due to a variety of stressors associated with increased anthropogenic activity on a global and local scale.

The marine environment is continuously exposed to change, but currently this change is more and more the result of human actions (Harvell et al. 1999; Derraik 2002; Orr et al. 2005; HoeghGuldberg and Bruno 2010). The stress exerted by the natural and anthropogenic induced changing global environment works in synergy with stressors that act on a finer spatial scale. Factors such as the overharvesting of fish, pollution, eutrophication, coastal development and the introduction of invasive species can locally trigger shifts in community composition and 
trophic hierarchy (Hughes 1994; Hughes et al. 2003; Pandolfi et al. 2003; Hughes et al. 2007; Hughes et al. 2017). By destabilising ecosystem functioning and interactions between key species, these stressors reduce reef resilience and therewith the capacity of coral reefs to cope with globally induced sea surface temperature anomalies or ocean acidification (Pandolfi et al. 2003; Bellwood et al. 2004; Hughes et al. 2017). Reefs in the wider Caribbean region seem particularly vulnerable to anthropogenic impact (Jackson et al. 2014). By large this can be ascribed to increased local pressures associated with the unprecedented human population expansion in the region. Since the 1950s, the total population in the Caribbean has more than doubled (United Nations, Department of Economic and Social Affairs, Population, Division, 2015). Natural biological and hydrological conditions are also less favourable compared to, for instance, the Indo-Pacific region (Roff and Mumby 2012). Biological diversity in the IndoPacific exceeds 10-fold the diversity found in the Caribbean (Spalding et al. 2001; Hoeksema et al. 2017), implying limited functional redundancy in the latter (Bellwood et al. 2003; Bellwood et al. 2004; Jackson et al. 2014). In addition, the quality of Caribbean surface water is significantly impacted by discharge from major South-American rivers like the Amazon and Orinoco as well as the North-American Mississippi river. The residence time of the polluted and eutrophic water from these rivers, combined with run-off and sewage water from the numerous islands is relatively long in the Caribbean Sea due to its distinct basin-like morphological and hydrological features (Roff and Mumby 2012). As a consequence of the rapid anthropogenic alteration of the marine environment we now see an ecological degradation of Caribbean coral reef habitats that has not occurred for over 200.000 years (Pandolfi and Jackson 2006).

\section{History and status of Caribbean coral reefs}

First indications of reef formation by scleractinian corals in the greater Caribbean dates back over 50 million years (Budd 2000 and references therein). Ever since, the Caribbean has experienced major episodes of accelerated extinction and faunal turnover initiated by gradual climatic change (Newell 1971; Sheehan 1985; Copper 1988). Yet, between episodes of mass extinction, reef communities consistently displayed ecological stability with slow biological change, principally driven by spatially constrained evolutionary processes (Copper 1988; Sheehan 1996). The most recent dramatic change in faunal composition on Caribbean reefs appears to have occurred during the Plio-Pleistocene (4 - $1 \mathrm{Ma}$ ) (Budd et al. 1996). During this period the Caribbean became isolated from the Pacific Ocean by the final closing of the Isthmus of Panama ( 3.5 Ma) (Woodring 1954), which marked the evolutionary divergence of coastal marine faunas in both regions (Coates et al. 1992; Jackson et al. 1993). Fossil records infer that many Pliocene coral species (> 70\%) currently still found on Indo-Pacific reefs became locally extinct in the Caribbean and Acropora, Orbicella and Diplopia genera started shaping the shallow reef habitat (Budd et al. 1999). The subsequent ecological persistence of these species appears to have been interrupted only several decades ago by the hand of humans (Pandolfi and Jackson 2006). Although human activity has negatively impacted Caribbean reefs for centuries (Jackson 1997; Jackson et al. 2001; O'Dea et al. 2014; Cramer et al. 2017), the actual functional collapse of these systems appears to have started in the early 1980s (Hughes 1994; Gardner et al. 2003; Pandolfi et al. 2003; Bak et al. 2005a; Jackson et al. 2014; Hughes et al. 2017). 
The ecological degradation that currently characterizes Caribbean reef ecosystems is manifested by the almost complete extermination of the Acropora genus by the White Band Disease that started in the 1980s (Gladfelter 1982; Aronson and Precht 2001). Acropora palmata and Acropora cervicornis, the two species that represent the Acropora genus in the Caribbean, have dominated shallow-water reef habitats $(<15 \mathrm{~m})$ throughout the greater Caribbean for thousands of years (Pandolfi and Jackson 2006). The loss of these fast-growing species with distinct branching morphologies has resulted in a completely altered shallow-water seascape (AlvarezFilip et al. 2013). Similarly, a pathogen caused the mass die-off (> 95\%) of the ubiquitous Diadema antillarum sea urchin between 1981 and 1983, by which a prominent herbivore was almost entirely diminished (Sammarco et al. 1974; Bak et al. 1984). Both cases have critically affected reef functioning and are exemplary of the limited functional redundancy on Caribbean reefs (Bellwood et al. 2004). Human activity has reduced water quality, particularly in coastal areas, and thereby stimulated conditions in which pathogens prevail and the overall resilience of corals is reduced (Nyström et al. 2000; Aronson and Precht 2001; Vega-Thurber et al. 2014). Consequently, these weakened corals have become more vulnerable to disease and thermal stress (Wooldridge 2009). Global climate change brought about by human activity resulted in recent (1998, 2005, and 2010) widespread bleaching events which have significantly reduced coral cover on Caribbean reefs (Donner et al. 2007; Wilkinson and Souter 2008; Jackson et al. 2014). The decline in coral cover has been aggravated by the overharvesting of fish and the loss of $D$. antillarum which has gravely reduced herbivorous pressure (Sammarco 1982; Bak et al. 1984; Lewis 1986; Hughes et al. 2007; Edwards et al. 2014). Simultaneously, reduced coral growth and the increased impact of bioerosion unbalanced net carbonate production, severely impacting the vertical accretion potential of many reefs. All together, the rapidly changing reef environment appears to have initiated the collapse of essential ecosystem functions and trophic cascades and has caused major shifts in benthic community configurations (Hughes 1994; Jackson et al. 2014; Hughes et al. 2017).

\section{Objectives and aim of this thesis}

Many scientific publications describe the various facets of the ecological changes that have occurred on Caribbean coral reefs over the past decades. The aim of this thesis is to contribute to the existing knowledge in order to present a more holistic view on the metamorphosis from pre-1980 reef habitats to reef configurations as we encounter them today. A more complete understanding will aid in designing appropriate monitoring projects and management objectives. This thesis focusses on two major aspects of the changing reef, which are in many ways heavily intertwined. The first focus lies on the temporal and spatial development of the most significant benthic sessile components. Together with the co-authors, I have intended to elaborate on the question: what major shifts have occurred within benthic coral reef communities? Based on time-series I describe the trajectories of change of the collective reef assemblage since the early 1970s and hypothesize on the impact that these changes have on the general condition of a reef. A comprehensive evaluation is provided, presupposing which organisms and species can currently be considered ecological "winners" or "losers" under various degrees of anthropogenic disturbance. The second major scope of this thesis 
concentrates on the declining deposition of calcium carbonate following the observed shifts in benthic community compositions. Are Caribbean coral reefs still able to achieve net vertical accretion? And if not, what consequences does this have for reef functioning and for us? Throughout this thesis, I tentatively elaborate on the link between increased human activity and the observed changes within reef communities and hypothesize on the question why certain areas appear to experience more or less ecological degradation. Finally, this thesis aims to highlight, where possible, signs of hope for the reefs in the wider Caribbean region. The work presented here is focussed on the fringing reefs of the southern Caribbean islands of Curaçao and Bonaire (formerly part of the Netherlands Antilles: Box 1.1), reefs that allegedly rank among the least degraded throughout the greater Caribbean region (Jackson et al. 2014).

\section{Outline per chapter}

Chapter II '40 Years of benthic community change on the Caribbean reefs of Curaçao and Bonaire: the rise of slimy cyanobacterial mats' provides an extensive assessment of changes in the composition of benthic sessile communities since the early 1970s. Ancillary to scleractinian corals and fleshy macroalgae, this chapter also describes the trajectories of change for crustose coralline algae, algal turfs, sponges and, benthic cyanobacterial mats. The temporal dynamics of these six major sessile constituents as well as abiotic components (sand, bare substratum) are compared among the studied reef sites and across an incorporated depth gradient from $10 \mathrm{~m}$ to $40 \mathrm{~m}$.

\section{Box 1.1}

The former Netherlands Antilles was an autonomous country within the Kingdom of the Netherlands. Initially, this Caribbean island group consisted of the Benedenwindse Eilanden: Aruba, Bonaire and Curaçao (ABC-islands) and the Bovenwindse Eilanden: Saba, Sint Eustatius and the Dutch side of Sint Maarten (SSS-islands). In 1986, Aruba left the Netherlands Antilles to become a separate country within the Kingdom of the Netherlands. Subsequently, after an official dissolution on the $10^{\text {th }}$ of October 2010, Bonaire, Sint Eustatius and Saba became special municipalities of the Netherlands (now referred to as the BES Islands or Caribbean Netherlands), while Curaçao and Sint Maarten became constituent countries within the Kingdom of the Netherlands, much like Aruba.

The historical ties between these Caribbean islands and the Kingdom of the Netherlands has encouraged longstanding marine and terrestrial research efforts. This has, among other things, resulted in the founding of the Caribbean Marine Biological Institute (CARMABI) on Curaçao in 1955 and the Caribbean Netherlands Science Institute (CNSI) on Sint Eustatius in 2014. The Kingdom of The Netherlands has, together with the local authorities, both national and international responsibility to safeguard the exceptional biodiversity and ensure adequate management of the natural resources within the exclusive economic zone of these islands. The research presented in this thesis was conducted at the request of Wageningen Marine Research (WMR) and the Royal Netherlands Institute for Sea Research (NIOZ) and with additional funding from the Ministry of Economic Affairs for this purpose. 
Chapter III 'Long-term Shifts in coral communities on shallow to deep reef slopes of Curaçao and Bonaire: are there any winners?' examines the spatio-temporal dynamics of coral species configurations on the reef slopes of Curaçao and Bonaire making use of the 40-year photoquadrat time series as described in chapter II. The status and development of the coral assemblage is assessed based on multiple characteristics including coral cover, number of coral species, colony size, colony shape and gross carbonate production.

Chapter IV, 'Quantification of chemical and mechanical bioerosion rates of six Caribbean excavating sponge species found on the coral reefs of Curaçao' describes the bioeroding potential of the six most prominent excavating sponge species on Curaçaoan reefs: Cliona caribbaea, C. aprica, C. delitrix, C. amplicavata, Siphonodictyon brevitubulatum and Suberea flavolivescens. Excavating sponges erode their host substratum through a combination of chemical dissolution and mechanical removal of $\mathrm{CaCO}_{3}$ fragments. Various conventional and novel methodologies to quantify both erosion components are tested and compared.

In Chapter V, 'Spatial variation in coral reef accretion potential poses significant concern for low-lying tropical coastlines', island-scale spatial variation in net carbonate production and vertical reef accretion potential is determined for 115 sites on the leeward reef of Bonaire. Estimates for net reef accretion were based on gross biogenic accretion and gross bioerosion rates quantified following the ReefBudget and maximum reef accretion methodologies (Perry et al. 2012; Perry et al. 2013). Accretion potential and local environmental conditions are used to conduct a risk assessment targeting areas prone to inundation with respect to projections for future sea level rise (IPCC 2018: RCP scenarios).

Chapter VI 'Towards stabilization of the decline in coral cover and fish biomass on the shallow reefs of Bonaire, Netherlands Caribbean?' describes the current status of benthos and fish communities on the reefs of Bonaire at high spatial resolution. The substantial variability in human activity along the leeward coast of Bonaire is expected to cause a gradient of local stress exerted on the adjacent fringing reef, resulting in the development of distinct reef assemblages that may be representable for reefs throughout the wider Caribbean.

In Chapter VII, 'General discussion: is there hope for Caribbean coral reefs?', the major findings of Chapter II to Chapter VI are thoroughly discussed in light of the objectives that have been proposed here in Chapter I. A concluding overview is presented of the status and major trends on the coral reefs of Curaçao and Bonaire. The aim of this chapter is to place the presented observations within the wider spectrum of coral reef research in the hope that they may aid in drafting appropriate management plans to maintain Caribbean reefs. 


\title{
CHAPTER II \\ 40 years of benthic community change on the Caribbean reefs of Curaçao and Bonaire: the rise of slimy cyanobacterial mats
}

\author{
Didier M. de Bakker, Fleur C. van Duyl, Rolf P. M. Bak, Maggy M. Nugues, \\ Gerard Nieuwland and Erik H. Meesters
}

Coral reefs, 2017: 36(2), 355-367

DOI: https://doi.org/10.1007/s00338-016-1534-9

Received the award for 'Best paper published in Coral Reefs in 2017'

\begin{abstract}
Over the past decades, numerous studies have reported declines in stony corals and, in many cases, phase shifts to fleshy macroalgae. However, long-term studies documenting changes in other benthic reef organisms are scarce. Here, we studied changes in cover of corals, algal turfs, benthic cyanobacterial mats, macroalgae, sponges and crustose coralline algae at four reef sites of the Caribbean islands of Curaçao and Bonaire over a time span of 40 years. Permanent $9 \mathrm{~m}^{2}$ quadrats at 10,20,30 and $40 \mathrm{~m}$ depth were photographed at 3 to 6-year intervals from 1973 to 2013. The temporal and spatial dynamics in the six dominant benthic groups were assessed based on image point-analysis. Our results show consistent patterns of benthic community change with a decrease in the cover of calcifying organisms across all sites and depths from $32.6 \%$ (1973) to $9.2 \%$ (2013) for corals and from $6.4 \%$ to $1 \%$ for crustose coralline algae. Initially, coral cover was replaced by algal turfs increasing from $24.5 \%$ (1973) to $38 \%$ around the early 1990s. Fleshy macroalgae, still absent in 1973, also proliferated covering $12 \%$ of the substratum approximately 20 years later. However, these new dominants largely declined in abundance from 2002 to 2013 (11\% and 2\%, respectively), marking the rise of benthic cyanobacterial mats. Cyanobacterial mats became the most dominant benthic component increasing from a mere $7.1 \%$ (2002) to $22.2 \%$ (2013). The observed increase was paralleled by a small but significant increase in sponge cover $(0.5 \%$ to $2.3 \%)$. Strikingly, this pattern of degradation and phase change occurred over the reef slope down to upper-mesophotic depths of $40 \mathrm{~m}$. These findings suggest that reefs dominated by algae may be less stable than previously thought and that the next phase may be the dominance of slimy cyanobacterial mats with some sponges.
\end{abstract}




\section{Introduction}

Over the past decades, benthic sessile communities of tropical coral reefs have undergone significant changes worldwide as a consequence of continuous human population expansion and industrial development (Hughes 1994; Gardner et al. 2003; Hughes et al. 2003). The synergistic effects of anthropogenic disturbances such as overfishing, coastal development, pollution and climate change have triggered shifts in benthic species composition, competition, productivity and biodiversity (Hughes 1994; Jackson et al. 2014; De Bakker et al. 2016b). The Caribbean region and Gulf of Mexico have been particularly affected with an estimated decrease in scleractinian coral cover up to $80 \%$ on many reefs. This is from an average of $35 \%$ cover in the 1970 s to a mean of only $14-16 \%$ between 0 and $20 \mathrm{~m}$ depth in more recent years (Gardner et al. 2003; Wilkinson and Souter 2008; Jackson et al. 2014). Over the same time span, benthic fleshy macroalgae have become a dominant component on many reef slopes (Hughes 1994; McCook et al. 2001; Nugues and Bak 2008), with an average increase in cover from $7 \%$ to $23 \%$ (Jackson et al. 2014). This phenomenon is commonly referred to as a 'coral-macroalgal phase shift'. The dynamics of both corals and macroalgae, as well as their competitive interactions, have received significant attention (e.g. Hughes 1994; McCook et al. 2001; Nugues and Bak 2008; Bruno et al. 2009). However, long-term studies following changes in benthic reef components other than stony corals and macroalgae are scarce.

The relative absence of studies including sessile organisms such as sponges, crustose coralline algae (CCA), gorgonians and benthic cyanobacterial mats (BCM) highlights a lack of information on a large proportion of the reef benthos. A more holistic view of the coral reef benthos could provide useful insights into the structure and functioning of reef communities. Additionally, the vast majority of coral community studies cover only the shallow zone of the fore-reef. Communities below $20 \mathrm{~m}$ depth have largely been neglected in temporal studies, even though these reefs are hypothesized to play a key role in reef resilience, functioning as a possible refuge or buffer for various coral reef organisms (Glynn 1996). The few existing temporal studies that have included reefs below $20 \mathrm{~m}$ indicate that reef degradation also occurs on upper mesophotic reefs (down to $40 \mathrm{~m}$ ) (e.g. Bak et al. 2005; Nagelkerken et al. 2005; Nugues and Bak 2008; De Bakker et al. 2016b).

The exact mechanisms driving coral reef degradation are likely impelled by a complex mixture of confounding anthropogenic and natural drivers. Increased eutrophication, sedimentation and decreased herbivory are among the most commonly designated factors reducing the ability of corals and CCA to cope with the various stressors impacting coral reefs (Littler and Littler 1998; Kuffner et al. 2008). Chronic nutrient enrichment increases the severity and prevalence of diseases and bleaching in corals (Bruno et al. 2003; Vega Thurber et al. 2014) and sedimentation due to land clearing and other disturbance of soil smothers corals (Rogers 1990; Nugues and Roberts 2003a; Nugues and Roberts 2003b). In addition, the massive die-off of the grazing sea urchin Diadema antillarum (Bak et al. 1984) around 1983 and continuous overharvesting of herbivorous fishes (Jackson et al. 2001) has significantly reduced grazing pressure on fleshy macroalgae in many Caribbean islands (Mumby et al. 2006). It is recognized that these processes 
and events have altered reef condition in favor of more adaptive, fast-growing organisms such as macroalgae (Hughes 1994; Bruno et al. 2009).

Besides macroalgae, algal turfs appear to benefit from a changing reef environment, in particular from eutrophication (Gorgula and Connell 2004; Vermeij et al. 2010). Due to their opportunistic life-history characteristics, turfs are able to rapidly occupy newly available substratum and as such algal turfs have become the dominant cover on many coral reefs worldwide (e.g. Gorgula and Connell 2004; Sandin et al. 2008b; Haas et al. 2010). Algal turfs are multispecies assemblages of algae and cyanobacteria that can undergo successional patterns (Connell et al. 2014). They can shift to macroalgal stands when herbivore pressure is relatively low, but also to BCM (Bender et al. 2014).

BCM appear to benefit from increased levels of eutrophication as well (Brocke et al. 2015a) and possibly, from selective herbivore browsing because the production of nitrogenous secondary metabolites makes them largely unpalatable (Thacker et al. 1997; Nagle and Paul 1998). Several studies describe the occurrence of harmful blooms, particularly of thermo-tolerant species (e.g. Hallock 2005; Paul et al. 2005; Paul 2008; Smith and Schindler 2009; Charpy et al. 2012). Dense cyanobacterial mats can have a variety of negative effects on reef communities: they inhibit recruitment (Kuffner et al. 2006), act as pathogens (Carlton and Richardson 1995), overgrow and smother reef benthos (Ritson-Williams et al. 2005; De Bakker et al. 2016a), create an anoxic environment (Brocke et al. 2015b) and produce chemicals that cause coral and fish mortality (Nagle and Paul 1998). Thus, an increase in the frequency of BCM blooms could significantly affect benthic reef communities.

Sponges are another benthic group abundantly present on coral reefs, but often overlooked in time-series studies. Especially in the Caribbean, they can exceed all other benthic components in cover and biomass and are in many aspects essential reef components (e.g. Diaz and Rützler 2001; De Goeij et al. 2013). Sponge cover appears to have remained relatively steady for a long time period (Nagelkerken et al. 2005). However, new studies describe a recent increase in sponge densities (e.g. Loh et al. 2015; McMurray et al. 2015), in particular coral-excavating sponges (Ward-Paige et al. 2005; Carballo et al. 2013). Although sponges are generally not able to rapidly colonize newly available substratum, they are strong competitors for space. In many cases, they actively overgrow and kill other benthic organisms (Pawlik et al. 2007). It is hypothesized that sponges might profit from increased macroalgal densities, feeding on the dissolved organic carbon they release (Mueller et al. 2014; Pawlik et al. 2016; Rix et al. 2016). This, in combination with a reduction of spongivores due to overfishing, may give a competitive advantage over reef building corals (Loh et al. 2015).

We studied the temporal development of the most significant benthic components including hard coral, fleshy macroalgae, crustose coralline algae, algal turfs, benthic cyanobacterial mats and sponges using the longest running (> 40 year) coral reef time series (Bak et al. 2005; De Bakker et al. 2016b). Our objectives were to (1) elaborate on consistencies and variations in 
benthic coral reef community dynamic at four Caribbean reef sites over the fore-reef slope from 10 to $40 \mathrm{~m}$ depth and (2) follow temporal trajectories of major individual benthic sessile groups.

\section{Materials and Methods}

\section{Site description}

Three sites on the leeward reef of Curaçao (Carmabi Buoy One [I and II] and Carmabi Buoy Two [III]) and one site on the leeward reef of Bonaire (Karpata [IV]) (Figure 2.1) have been visited since 1973 (1974 for Karpata) at intervals of $3-6$ year. At each site four permanent square quadrats $\left(9 \mathrm{~m}^{2}\right)$ were arranged along a transect at 10, 20, 30 and $40 \mathrm{~m}$ depth. During a survey, overview and detail $\left(\sim 1 \mathrm{~m}^{2}\right)$ photographs were collected to allow for detailed benthic community analysis and identification. The time period of 40 years includes the D. antillarum mass die-off in 1983, multiple mass bleaching events in the southern Caribbean (including 1998, 2005 and 2010) and several tropical storms (Joan: 1988, Lenny: 1999, Ivan: 2004, Omar: 2008). Detection of sponges, CCA and cyanobacteria required photographs of the highest quality. This limited the photographs available for analysis to 10 surveyed years between 1973 and 2013 (see Figure 2.2 for exact years). Most pictures were collected using a Nikonos-V underwater camera followed by a Nikon D300 and a Nikon D800, all with high quality lenses and power flashes.

\section{Benthic community analysis}

Overview images were de-skewed using the free transform option in Photoshop CS6 (Adobe Photoshop CS6 v13.0.1) to compensate for any picture angle distortion and conform to the original $9 \mathrm{~m}^{2}$ quadrats. Each overview picture was overlain with a 100-point $(10 \times 10)$ regular grid (e.g. Nugues and Bak 2008), and benthic cover - biotic and abiotic - underneath each individual point was determined in the software program Coral Point Count with Excel extension (CPCe) v4.1 (Kohler and Gill 2006). Detail pictures were used to resolve ambiguities. The following categories were assigned: live hard (scleractinian) coral [HC]; algal turfs (filamentous assemblages $<1 \mathrm{~cm}$ in height) [TF]; benthic cyanobacterial mats [BCM]; fleshy macroalgae [MA] (almost exclusively Lobophora spp. and Dictyota spp.); sponges [SP], further separated into massive and encrusting growth forms; crustose coralline algae [CCA]; bare substratum [BS] including recently dead coral, coral rubble and pavement; and sand [SD]. Gorgonians, hydrocorals (milleporids), tunicates (with the exception of Trididemnum solidum in 1989 at IV-20 m) and other invertebrates were extremely rare in the photographs and grouped under the category other [OT]. When it was impossible to identify a point, e.g. due to shading, it was classified as unidentifiable (25 of approximately 16,000 analyzed points). When a group was overgrowing another, only the overgrowing group was scored. The category BCM was only assigned when clear mats were visible. Distinguishing between turf and early cyanobacterial mats was challenging, especially in black and white images. This might have led in some cases to a minor underestimation of the cyanobacterial fraction and as a consequence our estimation of cover by BCM should be viewed as conservative. To test for the adequacy of the 100 points count method, we compared our results for coral cover with results obtained by digitizing the contour of each coral colony and adding all 2D colony surfaces for each quadrat. Both methods showed obvious similarity in cover and temporal patterns (Appendix 2.A). 


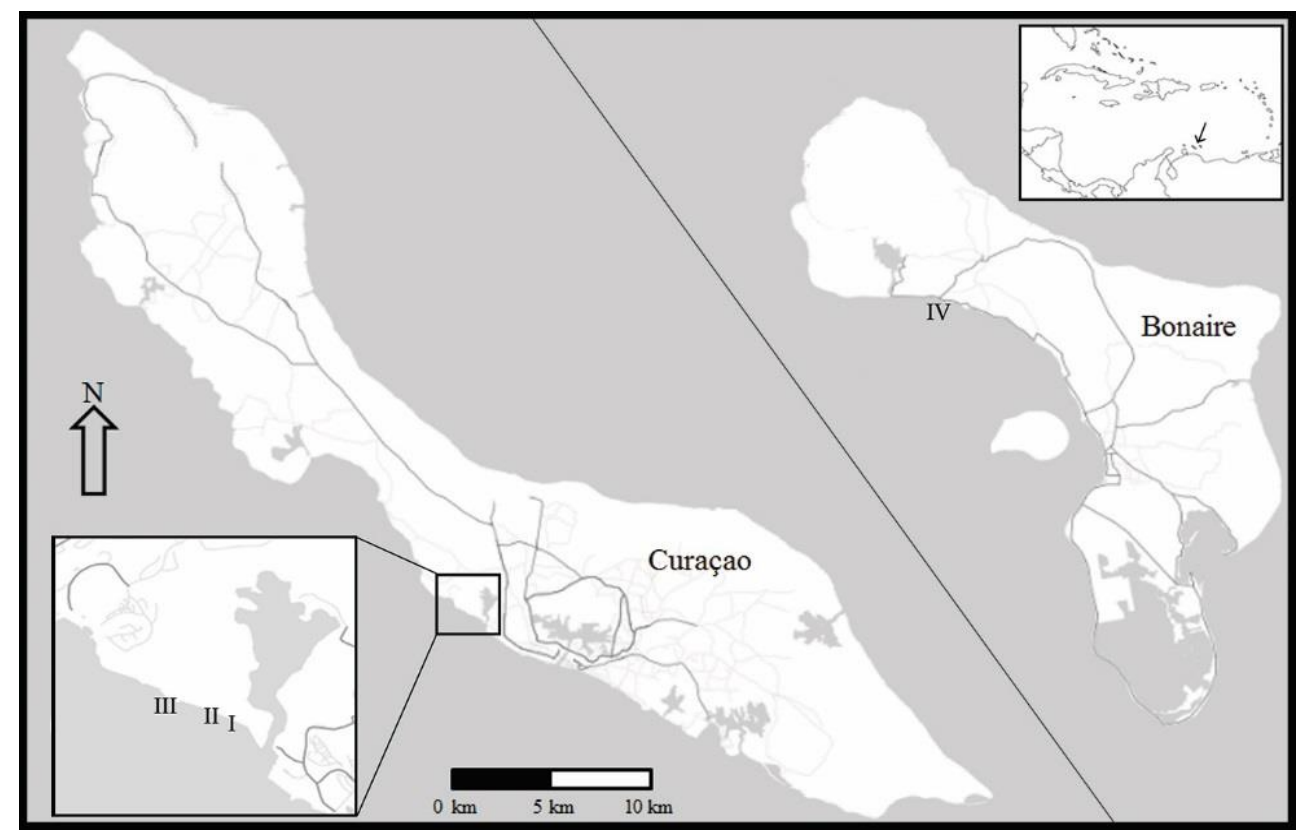

Figure 2.1. Overview of the survey sites at Curaçao (I-III) Bonaire (IV) and the position of both islands with respect to the wider Caribbean Sea. Scale bar applies to both islands.

\section{Statistical analyses}

Statistical analyses were conducted in the R programming environment v3.2.3 (R Core Team 2014) and Primer-E v7.0.9. Within $R$ the following packages were used: vegan (Oksanen et al. 2016) and $m g c v$ (Wood 2012). Statistical assumptions of univariate analyses were assessed through graphical exploration of residual errors.

\section{Multivariate analyses}

We applied multivariate analysis techniques (Gauch 1982) to visualise changes in community composition, based on a matrix of the original counts for the six most dominant biotic groups (HC, TF, BCM, MA, SP and CCA) with site (I, II, III, IV), depth (10, 20, 30 and $40 \mathrm{~m}$ ) and year $(\mathrm{n}=10)$ as factors. By omitting the category 'other' (due to low counts) and the non-biotic categories bare substratum (BS) and sand (SD) any collinearity among the remaining variables was removed. The point data were fourth-root-transformed to reduce the effect of high-cover groups. A Bray-Curtis similarity matrix (Bray and Curtis 1957) was computed between years, sites and depths. We explored temporal changes in composition of our benthic groups by nonmetric multidimensional scaling (nMDS). nMDS is a widely used method in ecological studies and provides good discrimination of multivariate patterns (Kruskal 1964; Clarke et al. 1993). In order to compare temporal linearity in multivariate space visually, years were displayed as points and joined in single nMDS plots for all respective quadrats (Figure 2.2). A seriation test was applied to test for linearity in the development of the community (Clarke et al. 1993). The 
RELATE routine in Primer-E tests the similarity between patterns of any two matrices of rank dissimilarities based on Spearman rank correlation coefficient (Kendall 1948), defined as the index of multivariate seriation (IMS) (Clarke et al. 1993). Linearity of the observed temporal patterns was assessed using this RELATE procedure $(M=9999$ simulations $)$ in which the correlation between the community distance (sub)matrix and a model matrix representing the distances in time between samples was calculated. A two-way RELATE test was used to assess correlation between patterns at different depths and sites by comparing submatrices of the individual quadrats $(M=9999$ simulations $)$.

In addition to the seriation tests, multivariate control chart analysis (Anderson and Thompson 2004) was applied to the Bray-Curtis matrices to determine when a community had become significantly different from the start of the time series (Appendix 2.B). The distance-based control chart method is a robust method that can be applied to sequential multivariate observations. In contrast to seriation, this method compares the community of each point in time to a set reference point, in our case the first survey in 1973 (Curaçao) or 1974 (Bonaire). Bootstrapping (1000 bootstraps) was used to provide percentile limits (50, 75, 90 and 95\%). Points positioned above the $90 \%$ limit were considered to have a significantly different community compared to the start of each time series (Anderson and Thompson 2004). R code for the multivariate control chart analysis was developed by EM.

\section{Univariate analyses}

Understanding the observed changes in the sessile community as a whole is only possible when looking at the temporal changes in the individual benthic components. We applied regression modelling to describe the historical trajectories of the six dominant benthic groups. Because ecological data, including our data, are often non-linear, generalized additive models (GAMs) were used to fit the data. GAMs are a non-parametric extension of generalized linear models (GLMs) and model the relationship between the response variable and the explanatory variables by means of smoothing curves (Chambers and Hastie 1992). Each benthic group was individually modelled with percentage cover as response variable for the explanatory variable 'year'. In total, four nested models were designed and tested for goodness of fit. The first model contained a smoothing term combining both depth and site $[f(\mathrm{DS})]$, leading to a total of 16 smoothers. Assuming analogy in patterns with depth or site, two less complicated nested models were designed having four smoothers for either depth or site. The simplest model had one smoother for time and the factors depth and site. Inference tests for selection of the best model were carried out by an $F$-ratio test (Appendix 2.C).

Various error distributions were applied to the GAMs and, although the resulting fits were comparable, models using transformed cover data with a normal (Gaussian) error distribution, identity link and a gamma value of 1.4 (Kim and $\mathrm{Gu}$ 2004) gave the most reliable and robust outcomes. Several other error distributions, for instance a beta distribution, caused an estimate bias when a series included many sequential zero values (BCM, MA, SP and CCA). Exploration of the model residuals with variogram plots indicated there was no need to account for temporal or spatial auto-correlation. 


\section{Investigating general patterns}

From the GAMs a new matrix was extracted with fitted values for each year for all 96 time series (i.e. a $40 \times 96$ matrix). A square-root transformation was applied. To remove the effect caused by large differences in percentage cover, fitted values were standardized on the basis of the maximum cover in each time series. Using Euclidean distance an nMDS plot was computed to display patterns in temporal development within and among the six main benthic groups. To reveal dominant clusters of time series with similar patterns a cluster analysis was run using the unweighted pair group method (Appendix 2.D).

\section{Results}

\section{Multivariate benthic community analyses}

The sessile biotic community on the studied reefs of Curaçao and Bonaire developed strongly unidirectional over the past four decades (Figure 2.2). The observed dynamics showed significant linearity in temporal development at all four sites and within sites at all four depths (Appendix 2.E \& 2.F). The nMDS plots displayed a regular distribution of points from left to right along the horizontal axis, very much following time. Patterns were homogenous among sites, but some variation among time series was found as well (e.g. III-40 and IV-40 m). Vector plots indicated the direction of the strongest correlation with the six main groups. The overall dynamics showed a pattern directed away from hard coral $(\mathrm{HC})$ and crustose coralline algae (CCA) and moving towards more benthic cyanobacterial mats (BCM), macroalgae (MA) and, below $10 \mathrm{~m}$, sponges (SP). Linearity in community change was most pronounced at $20 \mathrm{~m}$ for all sites $(P>0.76$; Appendix 2.E) and at $30 \mathrm{~m}$ particularly at sites $\mathrm{I}(P=0.82, p=0.001)$ and III $(P=0.83, p=0.001)$. Control charts confirmed a gradual change in community composition through time (Appendix 2.B). Both methods - seriation and control charts - indicated that significant reef community change was less pronounced at greater depths. Accordingly, moderate to weak but significant IMS scores were found at $40 \mathrm{~m}$. This is the case, for instance, at sites II $(P=0.39, p=0.01)$ and III $(P=0.36, p<0.01)$ which also clearly had a more narrow dispersion of points in the nMDS plots. Rank correlation of multivariate series indicated very similar patterns among depths in Bonaire $(P>0.64, p<0.05$; Appendix 2.C) and medium to high correlation between depths at the other sites. Values for within-site correlation were generally smaller than between sites at the same depth (Appendix 2.E \& 2.F), indicating that community responses were more structured by depth than by site. Significantly deviating correlations were found at $10 \mathrm{~m}$ depth between site II and both sites III $(P 2=0.24, p=0.10)$ and IV $(P 2=0.19, p=0.15)$ and at $40 \mathrm{~m}$ between Karpata (IV) and all other sites $(P 2<0.39$, $p>0.087$; Appendix 2.E). The latter indicated that there has been more variability in patterns of development at 10 and $40 \mathrm{~m}$ depth, compared to 20 and $30 \mathrm{~m}$. Here, community development was very consistent. Overall, the impact of local incidental disturbances (e.g. hurricanes, bleaching events, nutrient spikes), or seasonal variation (e.g. algal blooms during summer), was less pronounced. Because these effects may be temporary and the community might have the opportunity to recover, a response may fall outside the temporal and spatial detail of our time series. 
Table 2.1. Mean percentage cover and 95\% confidence intervals for the individual biotic and abiotic groups at this moment and at the start (1973, 1974 for Karpata) of the time series.

\begin{tabular}{l|c|c|c|c|}
\multicolumn{1}{c}{ Group } & 1973 & \multicolumn{3}{c}{$\mathbf{2 0 1 3}$} \\
\hline & Mean & $95 \%$ CI & Mean & $95 \%$ CI \\
\hline Hard coral & 32.6 & $(24.7-42.9)$ & 9.2 & $(5.3-14.5)$ \\
\hline Algal Turfs & 24.7 & $(18.4-32.1)$ & 10.7 & $(8.6-13.6)$ \\
\hline Benthic cyanobacterial mats & 0.1 & $(0.0-0.1)$ & 22.2 & $(16.0-29.5)$ \\
\hline Macroalgae & 0.0 & $(0.0-0.0)$ & 2.0 & $(0.4-6.6)$ \\
\hline Crustose coralline algae & 6.4 & $(4.4-9.0)$ & 1.0 & $(0.2-3.0)$ \\
\hline Sponges & 0.5 & $(0.1-1.5)$ & 2.3 & $(0.8-5.5)$ \\
\hline Other & 1.3 & $(0.2-4.3)$ & 2.3 & $(1.0-4.8)$ \\
\hline Bare substrate & 4.4 & $(2.9-6.4)$ & 0.6 & $(0.1-2.1)$ \\
\hline Sand & 13.8 & $(9.4-19.8)$ & 13.1 & $(9.2-18.0)$ \\
\hline
\end{tabular}

Figure 2.2. Non-metric multidimensional scaling ordinations for each individual quadrat (sitedepth combination). Plots are based on the six most dominant benthic groups: hard coral (HC), algal turf (TF), benthic cyanobacterial mats (BCM), macroalgae (MA), crustose coralline algae (CCA) and sponges (SP). Each survey is labelled with the last two numbers of the year of the survey. Lines represent the degree and direction of community change between two surveys. Vector plots are given in inset. Note MA was absent in I-10 m throughout the entire time series and is therefore not shown. 
Chapter II

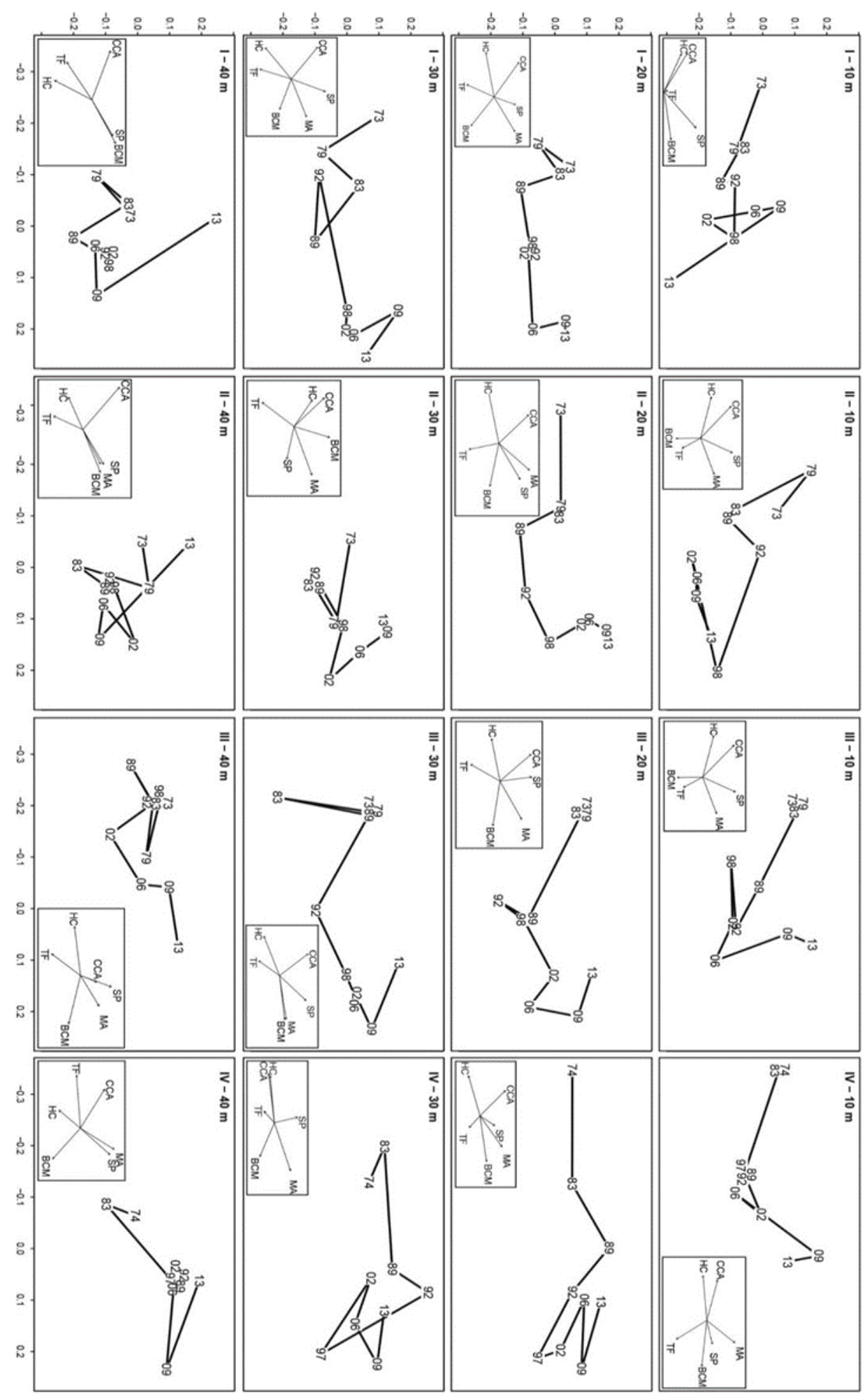




\section{Individual benthic groups}

\section{Scleractinian corals}

Between 1973 and 2013 coral cover in Curaçao and Bonaire declined by $71.8 \%$ over all sites and depths (Figure 2.3), from a mean of 32.6 to 9.2 (Table 2.1). Only at II-30 m did coral cover not decline significantly (Figure 2.3), but here cover was already relatively low in 1973 . Although coral cover declined universally, the trajectories varied between time series. At the shallow fore-reef $(10,20 \mathrm{~m})$ cover decreased by $79 \%$ over all quadrats between 1973 and 2013, while at deeper reefs, a slightly lower decline $(61 \%)$ was observed. In general, the decreasing trend in coral cover occurred gradually through time in a relatively linear pattern with some exceptions (Figure 2.3). The continuous decrease was only briefly interrupted by a period of apparent recovery between approximately 1992 and 2002 across the entire depth gradient of site I. At sites II and III at $40 \mathrm{~m}$ depth, coral cover initially increased until 1998, but subsequently decreased exponentially towards 2013. At $20 \mathrm{~m}$ in IV, coral cover declined exponentially between 1983 and 1992, likely due to the expansion of Trididemnum solidum (Bak et al. 1981; Van Duyl et al. 1981). Around the late 1980s T. solidum became very abundant on these reefs, particularly around $20 \mathrm{~m}$, covering up 26\% of the quadrat (Appendix 2.G), and actively overgrowing and killing corals and other benthic components (Bak et al. 1996). The GAM with 16 smoothers explained $97.4 \%$ of the deviance (adjusted $R^{2}=0.95$ ) and was always significantly better than all three alternative nested models (Appendix 2.C), which suggests considerable influence of local variables.

\section{Algal turfs}

Turf assemblages increased in cover from 1973, becoming the dominant biotic benthic component with a mean cover close to $40 \%$ over all quadrats around the late 1980 s to early 1990s. Subsequently, however, an often exponential decline in cover was observed starting around the late 1990 s towards a mean cover of $10.7 \%$ in 2013 . This is less than half of the initial cover of $24.7 \%$ (Table 2.1). Similar to corals, the GAM with 16 separate lines was the best model. With the second best model (same trends at depth), however, the difference was less convincing ( $p=0.02$; Appendix 2.C). In both shallow quadrats $(10,20 \mathrm{~m}$ ) of site I, cover of algal turfs did not show a significant change (Appendix 2.H). With the exception of these latter two quadrats and the almost linear decline at IV-40 m, the dynamic of algal turfs developed in a highly comparable fashion over all sites and especially within the same depth.

Figure 2.3. Temporal change in cover of the six dominant benthic groups between 1973 and 2013. Lines represent the estimated models (with 95\% confidence bands). Columns represent sites; I, II, III are in Curaçao, IV is in Bonaire. Rows represent depths. Black dots are original data points. Note that Y-axes (percentage cover) vary for the different benthic groups. Significance level of smoothers: $*<0.05, * *<0.01$. 
Chapter II
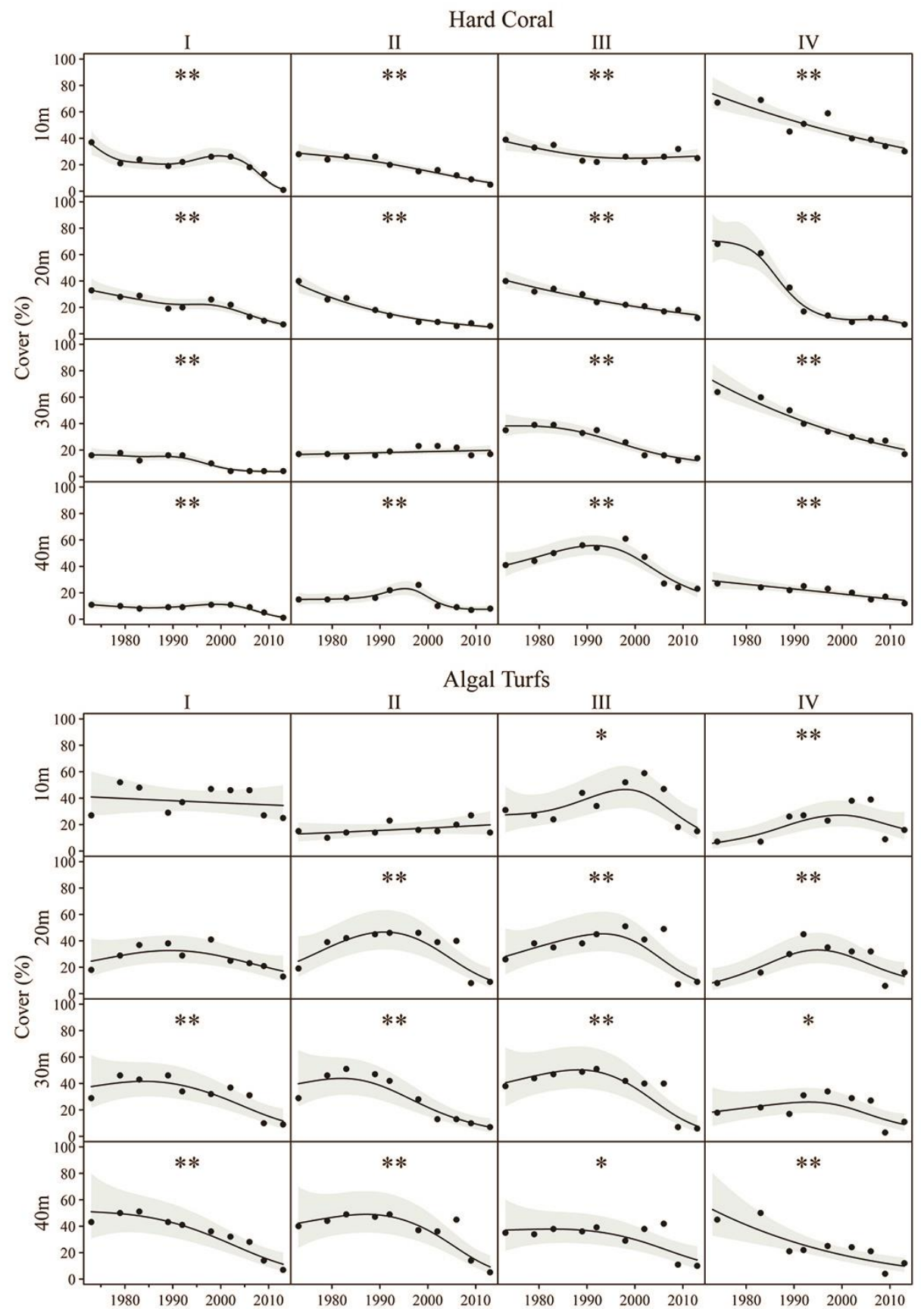

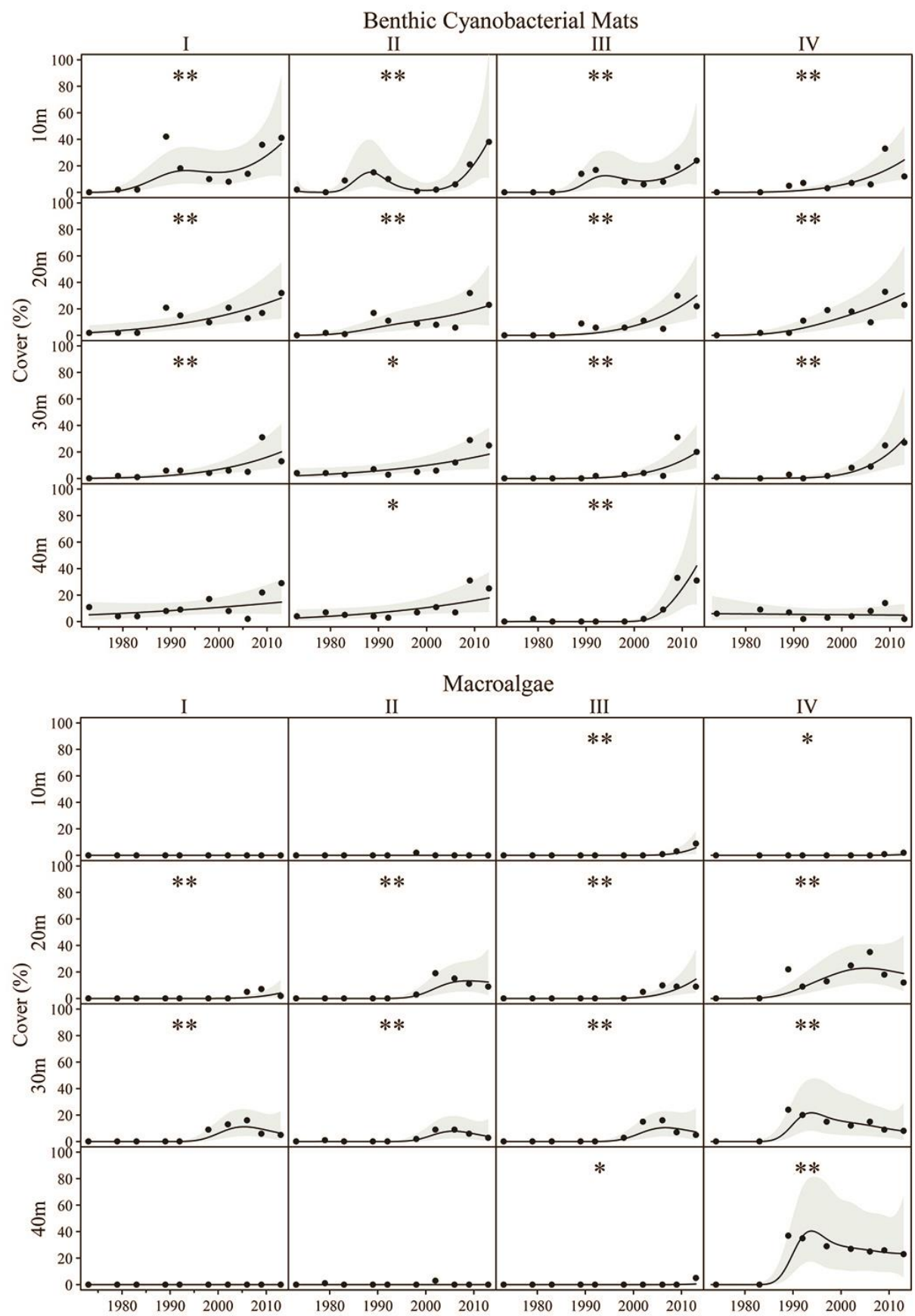
Chapter II
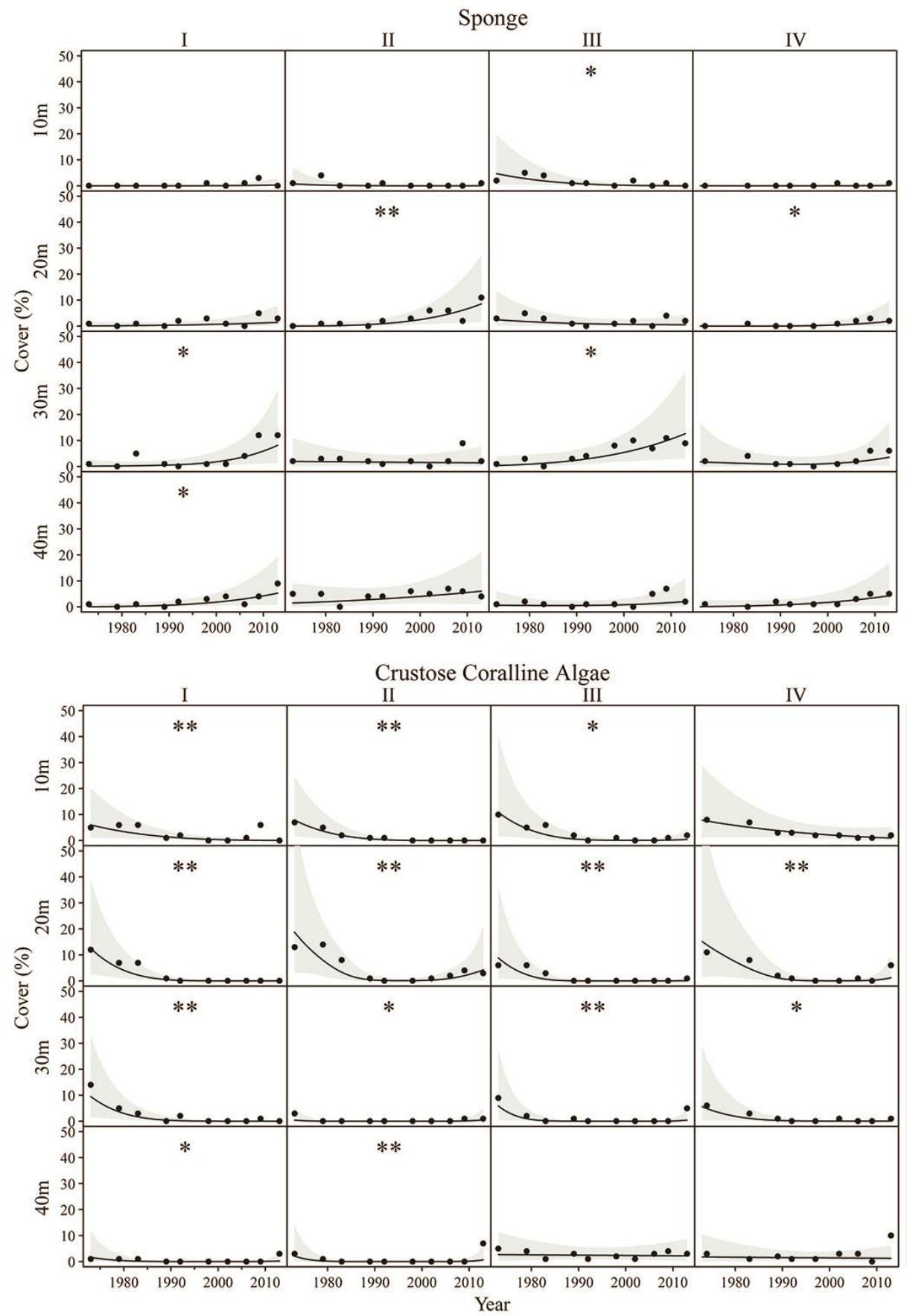


\section{Benthic cyanobacterial mats}

In recent years benthic cyanobacterial mats (BCM) have shown a striking increase at all sites and depths (Figure 2.3). At the start of the time series BCM were virtually absent covering a mere $0.1 \%$ (Table 2.1). Other than a small peak around 1989 on the shallow reef $(<20 \mathrm{~m})$ of Curaçao, particularly at $10 \mathrm{~m}$, the period preceding the rise in BCM is marked by a cover of less than $10 \%$ and an absence of any notable dynamics. After 2002, however, cover increased strongly, sometimes even exponentially, and by 2009 BCM had become the dominant biotic benthic group covering 22.2\% of the substratum (Appendix 2.G). GAM results showed that all but two (sites I-40 and IV-40 m) curves were significant (Appendix 2.G), suggesting a lack of change at these two deeper sites. The most complex model with 16 smoothers was the best model indicating differences in patterns of development between sites and depths.

\section{Fleshy macroalgae}

There were obvious differences among depths in temporal patterns of fleshy macroalgae (Figure 2.3). At $10 \mathrm{~m}$ macroalgae were almost absent while at 20 and $30 \mathrm{~m}$ the expansion on Curaçao started around 1998. On Bonaire (IV) macroalgae started increasing from 1983 onward. In addition, at $40 \mathrm{~m}$ depth, macroalgae on Bonaire reached high densities while they remained almost absent on Curaçao at this depth. Most significant trends showed a gradual decrease in cover after approximately 2002. With so many zero values, the approximate $\mathrm{p}$ values of the smoothers should be interpreted with caution. Values above 0.01 should probably be considered as not significant. The model with 16 different trends was the best model, confirming the highly variable trajectories between time series in macroalgal cover. A notable interaction between Dictyota spp. and benthic cyanobacterial mats was frequently observed, where one group was overgrowing the other. BCM often appeared to facilitate growth of Dictyota spp. on sand, possibly by stabilizing the sandy substratum. In various cases, however, BCM also actively overgrew and potentially smothered Dictyota spp. This interaction could contribute to the observed recent decline in macroalgal cover since we only incorporated the overgrowing component in our analyses.

\section{Sponges}

Although sponges were generally present at low cover, they increased in the last 10-15 year, particularly below $10 \mathrm{~m}$ depth (Figure 2.3). Sponge cover remained low between $1973(0.5 \%)$ and $1998(0.4 \%)$. After 1998 it increased almost six fold to 2.3\% in 2013 (Table 2.1). At $10 \mathrm{~m}$ depth, sponge cover was generally very low and patterns of change were highly variable among sites, slightly increasing at sites I and IV and decreasing at sites II and III. The model with 16 smoothers was the best model. Differences with models 3 and 4, however, were relatively small ( $\mathrm{p}=0.03$ ), indicating some similarity among sites. Massive and encrusting sponges both increased in cover between 1973 and 2013 by $74 \%$ and 62, respectively.

\section{Crustose coralline algae}

Similar to corals, CCA cover decreased during the first 20 year and remained very low to 2013 (Figure 2.3). Mean CCA cover over all quadrats decreased from 6.4 (1973) to only $1.0 \%$ (2013) (Table 2.1). Model 1 was significantly better than the three simpler models. 


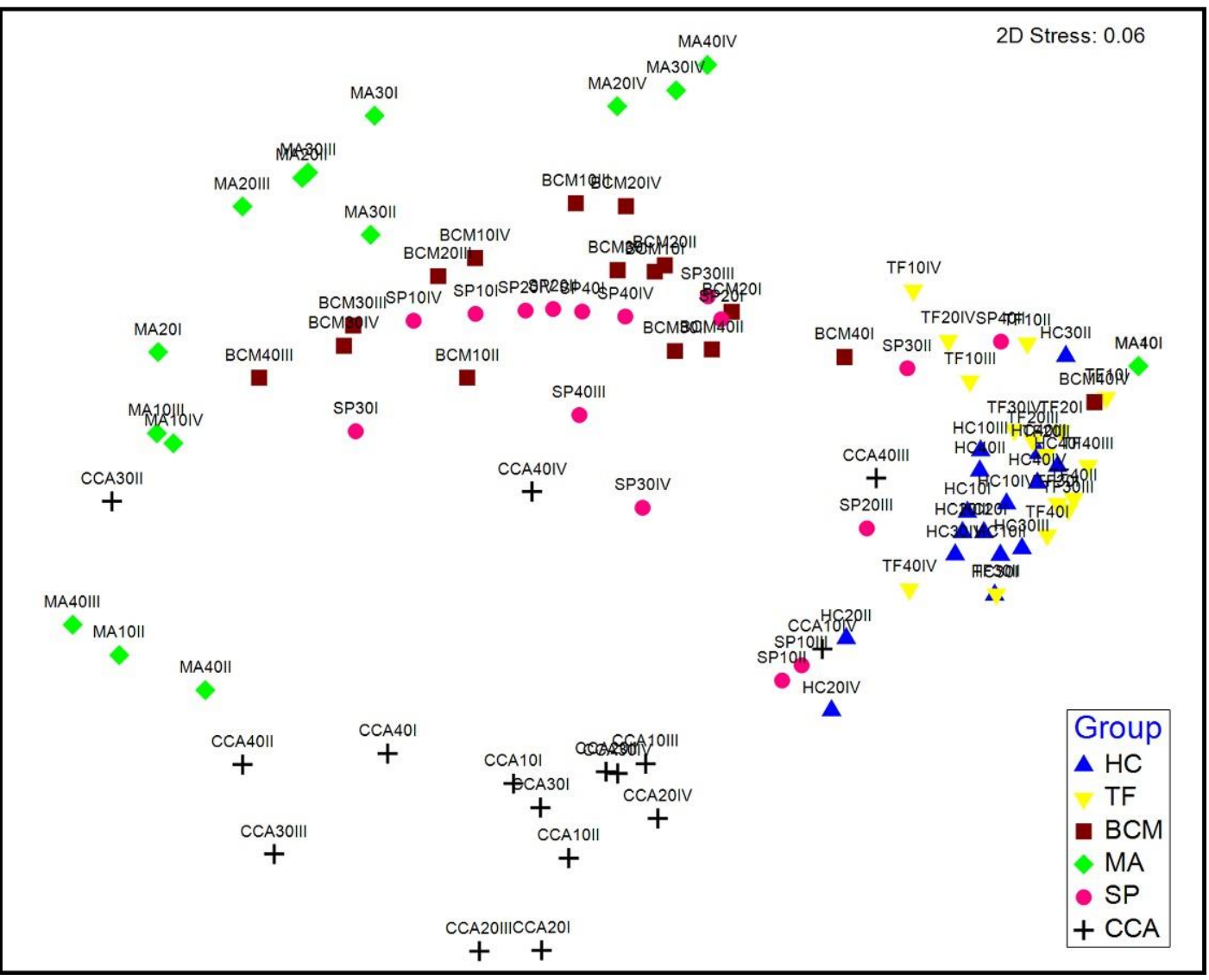

Figure 2.4. Non-metric multidimensional scaling plot displaying the degree of similarity between time series within and among the six main benthic groups: hard coral (HC, blue triangles), algal turfs (TF, yellow inverted triangles), benthic cyanobacterial mats (BCM, brown squares), macroalgae (MA, green diamonds), sponges (SP, pink circles) and crustose coralline algae (CCA, black crosses). Each point represents the pattern of changes in cover of a specific benthic category at one site and depth (i.e. an individual time series). Closer points indicate more similar patterns through time. The label of each point consists of the abbreviation of the benthic group, depth (10, 20, 30, $40 \mathrm{~m})$ and site (I, II, III, IV).

\section{Similarities and variation between time series}

Despite the large number of comparisons $(40 \times 96)$, time series formed distinct clusters per benthic group, in particular for $\mathrm{HC}, \mathrm{TF}$ and $\mathrm{BCM}$, which confirms similarity in the temporal trajectories of cover within each group (Figure 2.4). There was a clear clustering of groups that increased over time (BCM, MA, SP), as well as considerable overlap of groups that ultimately declined (HC, TF, CCA). The time series of SP and CCA were more scattered, but still relatively closely grouped (Figure 2.4). This suggests that patterns of change in these groups were largely consistent, but that local variation also existed. MA showed strongest diversity in patterns, with the deeper (> $10 \mathrm{~m}$ ) quadrats at site IV (Bonaire) clearly separated from the sites on Curaçao. 


\section{Discussion}

\section{Phase shifts on the reefs of Curaçao and Bonaire}

The composition of the benthic coral reef community in Curaçao and Bonaire between 10 and $40 \mathrm{~m}$ depth has changed significantly between 1973 and 2013. In general, the direction of the shift was similar across sites and depths (Figure 2.2), suggesting spatial homogeneity in the forcing factor(s) driving the trajectories of change in reef community composition. We have integrated temporal trends pooling sites and depths in Figure 2.5. Most striking is the unprecedented increase in cover of BCM from the late 1990s onwards. BCM have become a dominant component on these reefs at least down to $40 \mathrm{~m}$. The reefs on Curaçao and Bonaire are commonly classified among the least degraded in the Caribbean region (Jackson et al. 2014). Unlike Jackson et al. (2014), our sites only included reefs downstream or close to urban areas. In the 1970s the community was dominated by calcifying organisms (corals and CCA). Over a 40-yr period, it has developed towards a community dominated by algae and subsequently cyanobacterial mats. The obvious decline in coral cover and the increase in algal abundance from 1973 to 2000 match those observed on coral reefs throughout the wider Caribbean region (Gardner et al. 2003; Bak et al. 2005; Jackson et al. 2014) and worldwide (Hughes 1994; McCook et al. 2001). CCA also declined. Although CCA cover was generally low and fluctuated between time series, our results highlight a conspicuous loss in benthic calcifying organisms. Reduced calcification and cementation on a reef means a decline in reef accretion. Ultimately, this will lead to a further loss of reef structural complexity (Alvarez-Filip et al. 2011b).

Over the past 40 year coral cover gradually declined (Figure 2.3). This decline in coral cover is not clearly linked to a sudden increase in cover of any of the benthic competing organisms (TF, MA or BCM) and does not support competitive loss to a given benthic group as a primary cause of coral mortality. The observed shift towards fast-growing organisms may, however, constrain the ability of corals to reclaim invaded substratum and prevent coral recovery after disturbance (McCook et al. 2001). The absence of obvious fluctuations in cover of sand and bare substratum indicates rapid colonization of free substratum after coral mortality and suggests that competition for newly vacant space on hard substratum is intense. The increase in BCM coupled with a decline in algal turfs and, to a lesser extent, fleshy macroalgae, and an increase in sponge cover may indicate a new successional phase of coral reefs.

\section{The rise of $B C M$ and sponges}

The trend of algal turf assemblages becoming the dominant benthic cover on the reefs of Curaçao and Bonaire has been observed on reefs worldwide, e.g. Curaçao (Vermeij et al. 2010), Australia (Gorgula and Connell 2004), the Gulf of Arabia (Haas et al. 2010) and the remote Line Islands in the Pacific (Sandin et al. 2008b). The consensus is that algal turfs benefit from elevated nutrient loads (Gorgula and Connell 2004; Vermeij et al. 2010). It is often suggested that a phase shift has occurred on many coral reefs where algae have mostly replaced the calcifying organisms (e.g. Hughes 1994). From the late 1990s onwards, however, BCM increased and became dominant at the expense of algal turfs and macroalgae while sponges showed a more limited but steady and significant increase (Figure 2.3). The frequently observed 
interaction in which BCM overgrow fleshy macroalgae may have led to an underestimated recent algal cover. Despite being overgrown, these macroalgae (almost exclusively Dictyota spp. and Lobophora spp.) may still be functionally important in the benthic assembly. Nevertheless, macroalgae have declined in cover and the observed shifts suggest that former reefs recently dominated by algae may be less stable than previously thought and that a next successional phase may be dominated by BCM and sponges with some fleshy macroalgae.

Algal turfs consist of a large consortium of species, including cyanobacteria (Connell et al. 2014). They may develop to later successional taxa such as standing crops of macroalgae or BCM depending on local and global conditions. For instance, reduced water quality, high grazing pressure and elevated water temperature stimulate BCM growth over macroalgal growth (Kuffner and Paul 2001; Bender et al. 2014). Many cyanobacterial species can fix nitrogen (Larkum et al. 1988), but are limited in growth by phosphorous or iron (Kuffner and Paul 2001), indicating they may benefit from elevated nutrient levels in the water. They also respond positively to organic matter enrichment in sediments (Brocke et al. 2015a).

High BCM cover has been observed along the leeward side of Curaçao, Bonaire, and on multiple other reefs throughout the Caribbean including St. Eustatius, Martinique, and on Saba Bank (de Bakker et al. pers. obs.). In addition, high BCM cover has been observed throughout the entire year, suggesting high cover is not necessarily only seasonal. Increased occurrence of reef dwelling cyanobacterial blooms has been found on coral reefs around the world (e.g. Charpy et al. 2012). It is plausible that a decline in water quality and elevated water temperature initiated the recent shift to dominance of BCM on these reefs (Brocke et al. 2015a/b; Den Haan 2015). The reefs of both islands receive untreated sewage water via discharge and/or groundwater (Buth and Ras 1992; Lapointe and Mallin 2011). This probably applies to the entire course of the time series, because local sewage treatment plants are overall not working properly (Curaçao Ministry of Health, Environment and Nature 2014). A considerable rise in the number of inhabitants (50\% increase since 2001 in Bonaire) and tourists on both islands (CBS Curaçao, www.cbs.cw; CBS Dutch Caribbean, www.rijksdienstcn.com) has further increased the pressure of sewage pollution as well as land-based run-off and sedimentation on these reefs. Obtaining supporting evidence for such eutrophication is challenging because the largest pool of nutrients reaching the reef will be rapidly converted into biomass or trapped in the sediment (Koop et al. 2001). Slijkerman et al. (2014) and Govers et al. (2014) found indications of eutrophication on the reefs of Curaçao and Bonaire; however, long-term measurements of nutrient concentrations are virtually absent. Apparently, the entire Caribbean basin suffers from elevated nutrient load as a consequence of human impact (Siung-Chang 1997; Pawlik et al. 2016). Accordingly, increased anthropogenic induced stress both local (e.g. eutrophication) and regional (e.g. elevated temperature) likely initiated the observed community shift from algal dominance towards dominance of BCM and sponges. The descriptive nature of the data, however, allows only speculation with regard to the exact underlying causes behind the observed trends. 


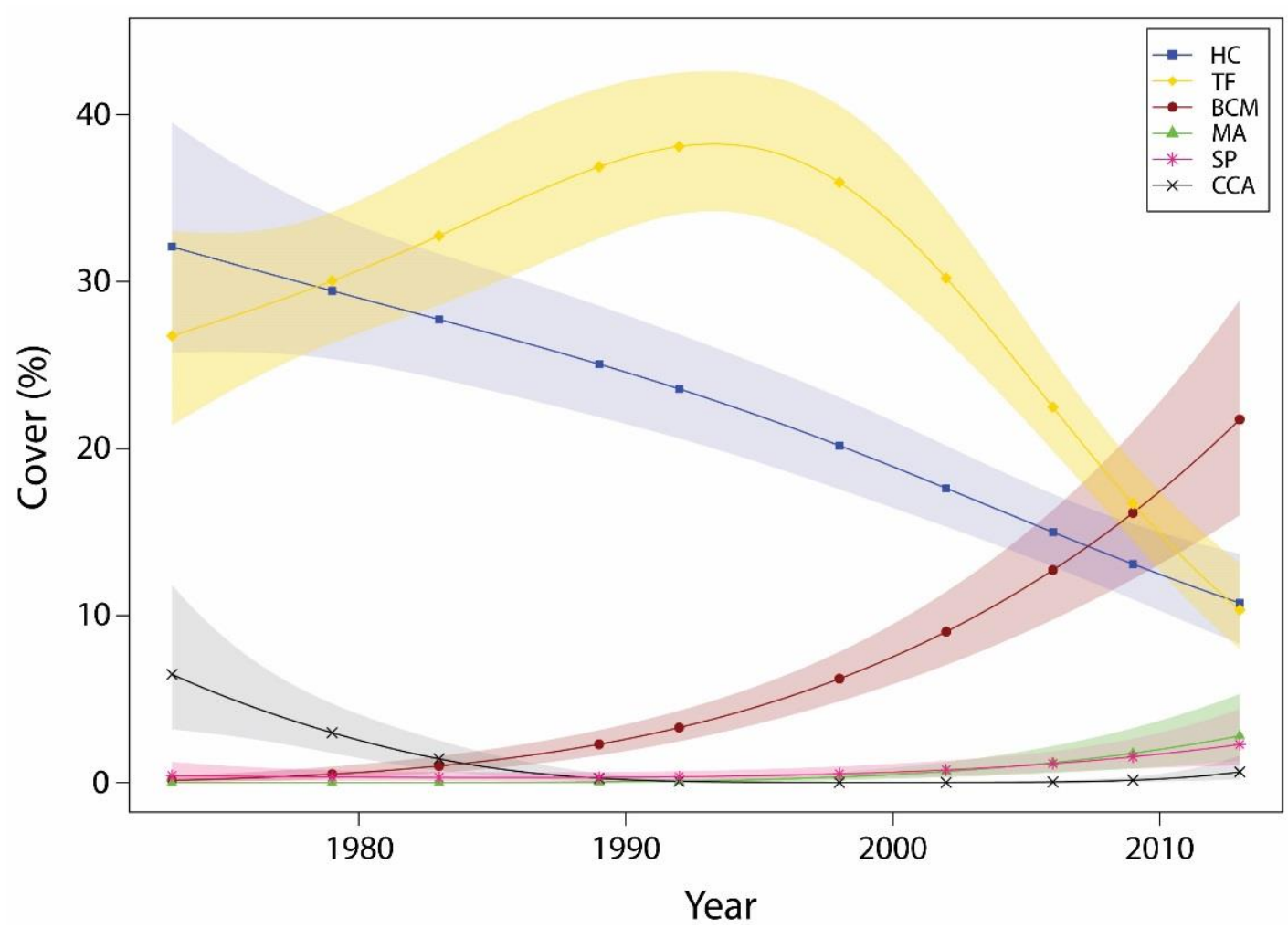

Figure 2.5. Trajectories of change for six benthic groups: hard coral (HC, blue), algal turfs (TF, yellow), benthic cyanobacterial mats (BCM, brown), macroalgae (MA, green), sponges (SP, pink) and crustose coralline algae (CCA, black). Lines represent estimated models (with 95\% confidence bands) of the change in mean percentage cover over all sites (I, II, III, IV) and depths $(10,20,30,40 \mathrm{~m})$.

Below $10 \mathrm{~m}$ depth, sponge cover started increasing at approximately the same time that BCM appeared on a large scale (Figure 2.5). Explanations for this increase in sponge cover could be the removal of spongivorous fishes (Loh et al. 2015) and/or a positive feedback loop between sponges, macroalgae and microbes on Caribbean reefs (Pawlik et al. 2016). Sponges feed on dissolved organic matter (DOM) produced by algae (Mueller et al. 2014; Rix et al. 2016). In turn sponges release nutrients (De Goeij et al. 2013) that can fuel macroalgal expansion. A similar feedback loop may exist involving BCM, which also release DOM (Brocke et al. 2015b). However, whether BCM-derived DOM is a suitable source for sponges remains to be investigated. Nevertheless, algae, BCM and sponges all compete for space with corals and can have negative consequences for the coral microbiome, reducing coral resilience to disturbances and reinforcing reef degradation (Vega Thurber et al. 2009; Pawlik et al. 2016). 


\section{Community dynamics across a depth transect}

Deeper reefs (> $20 \mathrm{~m}$ ) have rarely been considered in long-term ecological studies (but see Bak and Luckhurst 1980; Bak et al. 2005; Nagelkerken et al. 2005; Nugues and Bak 2008; De Bakker et al. 2016b). Our study shows that the observed main trends in community dynamic occurred down to $40 \mathrm{~m}$ depth. Although changes at $40 \mathrm{~m}$ depth were less pronounced (Figure 2.2), trends on the upper mesophotic reefs ( 30 and $40 \mathrm{~m}$ ) were also marked by a loss of calcifying organisms, mainly driven by the mortality of Agaricia species (Bak et al. 2005), and a shift towards BCM dominance (Figure 2.3). It is commonly assumed that deeper reefs are less subjected to anthropogenic impacts and events such as hurricanes, bleaching or harmful blooms and serve as potential refugia or buffer for coral reef species (Glynn 1996; Hughes and Tanner 2000). However, our study shows that upper mesophotic reefs are not free of stress (see also Bongaerts et al. 2010). For example, bleaching and increased sedimentation have also affected the deeper reefs of Curaçao and Bonaire (Bak et al. 2005; Nugues and Bak 2008). The degradation of communities on deep reefs suggests both reef zones (shallow and deep) are now subject to anthropogenic induces disturbances.

In conclusion, the composition of benthic communities on the fore-reef of Curaçao and Bonaire has changed dramatically over the past 40 year. Calcifying organisms have been reduced to mere figurants, and cyanobacterial mats have become a dominant benthic component. Our results suggest that the shift from coral and CCA towards turf and macroalgae may be a transitional phase that can further develop towards a new successional phase of BCM and sponge dominance with a less prominent role for fleshy macroalgae. This new community state has not previously been thoroughly described. Considering the ongoing increase of human impact (both local and global) on the coral reef environment, it may represent the next alarming step in the degradation of coral reef health. 


\section{Acknowledgement}

We are deeply grateful to the CARMABI research institute on Curaçao and STINAPA on Bonaire for their assistance over the years. The present research project (Ph.D. DdeB) was funded by Wageningen Marine Research, formerly known as IMARES, at the request of and with funding from the Ministry of Economic Affairs for the purposes of the Policy Supporting Research Theme 'Analyse fotomateriaal koraalrif/fase 1' (Project No. BO-11-019.02-038). The Royal Netherlands Institute for Sea Research provided funding of expenses and in-kind support. We would like to thank H. Groen and both reviewers for a thorough review of the manuscript. 


\section{Appendix 2.A}

Method comparison. A) Modelled patterns of development in hard coral cover over time based on the 100-point grid estimate and B) Modelled patterns of development in hard coral cover over time based on the actual total coral cover in the quadrats. Patterns are given for all 16 quadrats (I - IV) and all depths (10 - $40 \mathrm{~m}$ ). Significance level of smoothers: $*<0.05, * *<0.01$.
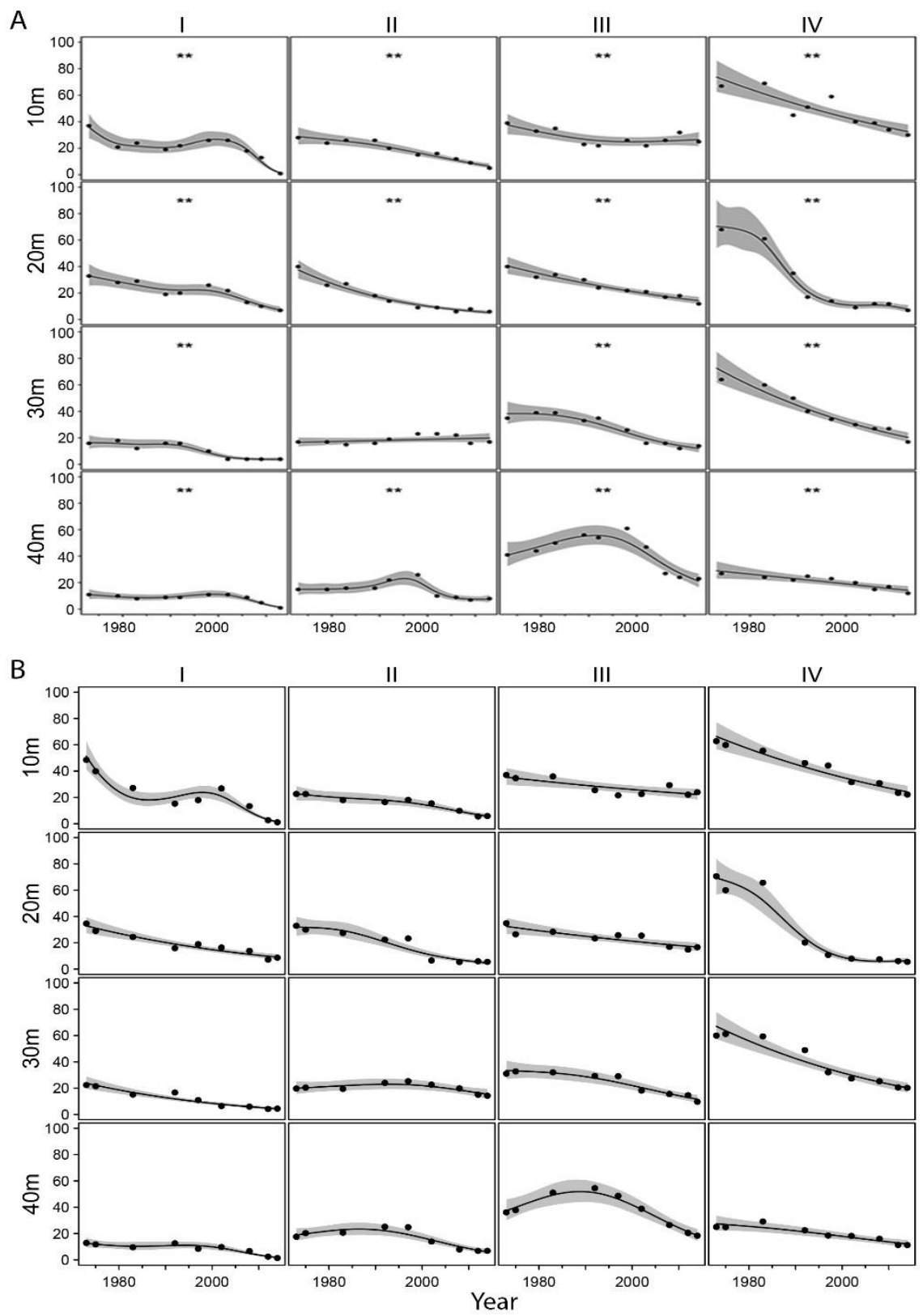
40 years of benthic community change on the reefs of Curaçao and Bonaire
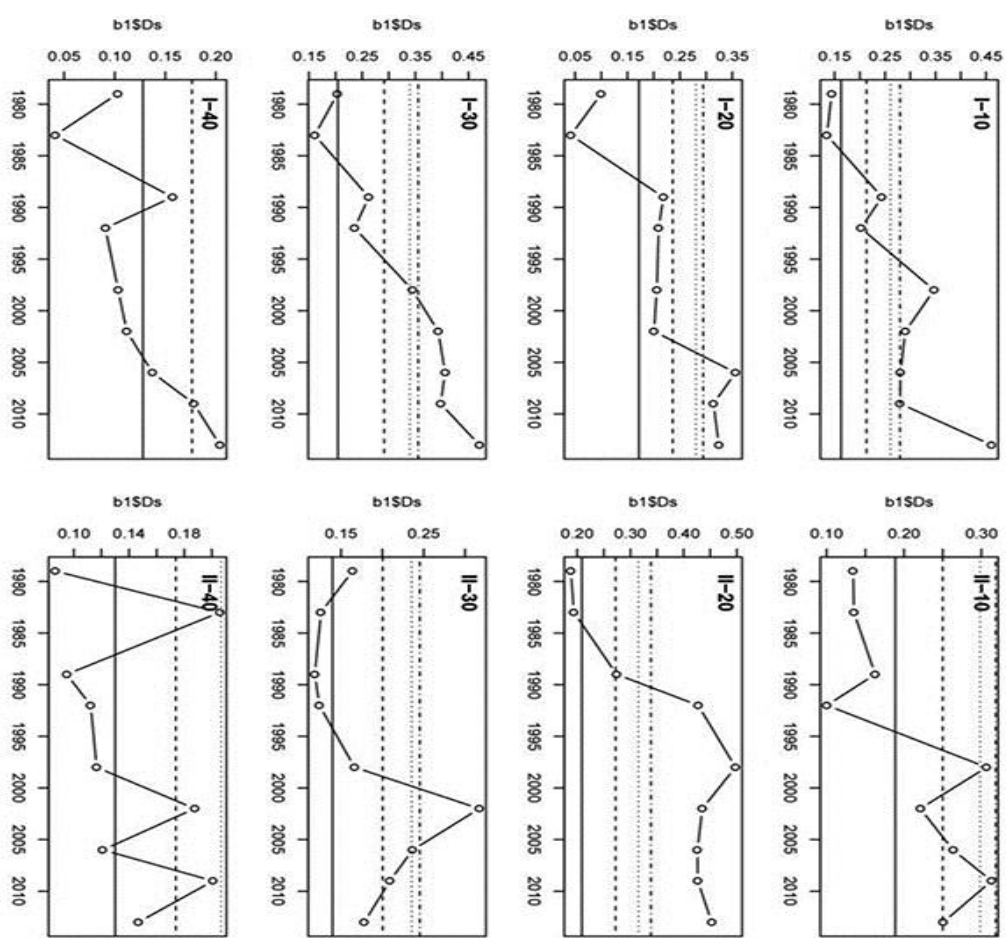

$\underset{\mathbb{9}}{\stackrel{\Upsilon}{2}}$
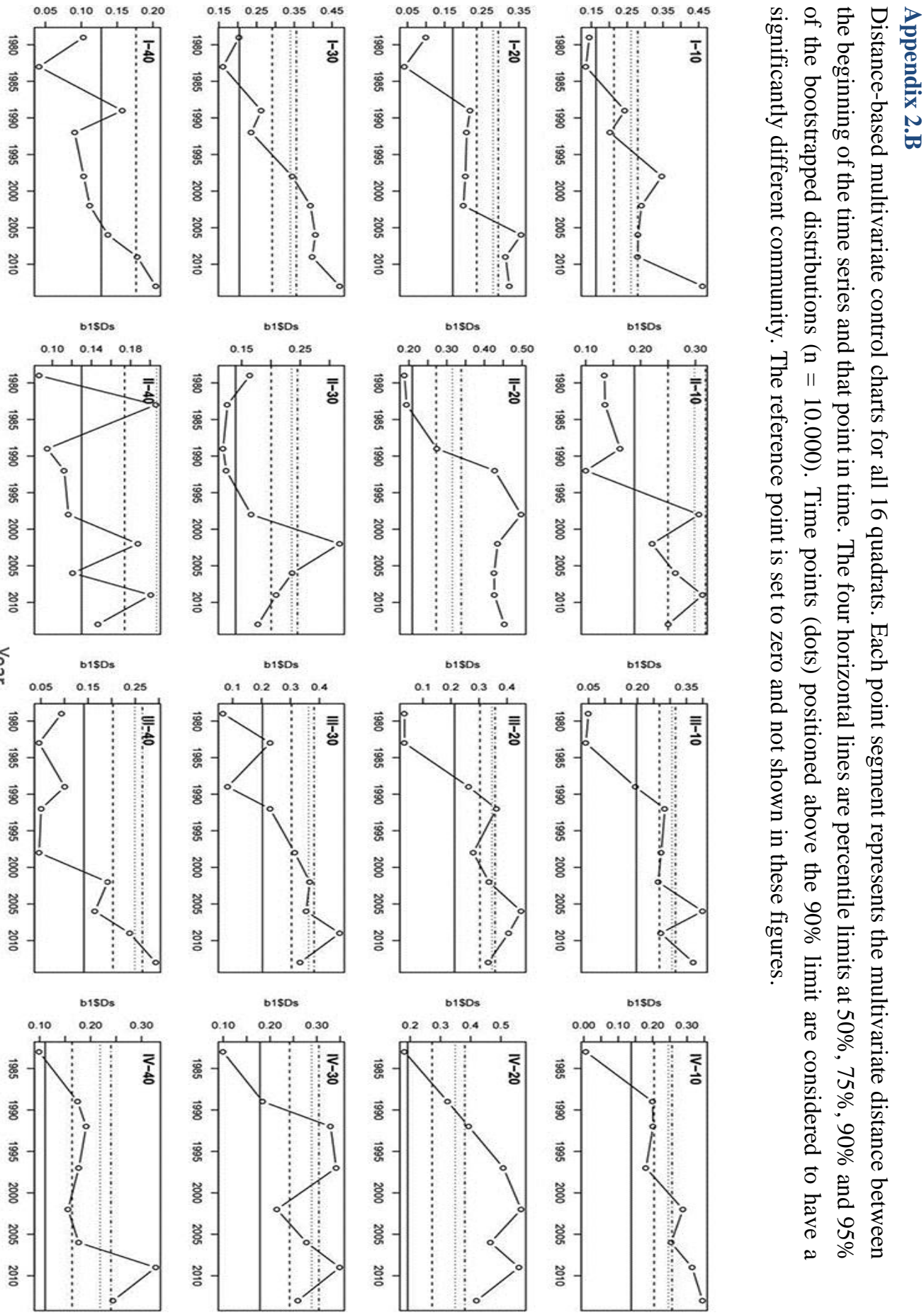
Appendix 2.C

GAM model comparison. $F$-test outcomes for comparison of the four different nested GAM models (mod1 - mod4) designed for each benthic group: hard coral (HC), algal turfs (TF), benthic cyanobacterial mats (BCMs), macroalgae (MA), sponges (SP) and crustose coralline algae (CCA), $p$-value below .05 indicate mod1 is the significantly better model.

\begin{tabular}{|c|c|c|c|c|c|c|c|c|c|}
\hline & Model & $\begin{array}{c}\text { Adj. } \\
R^{2}\end{array}$ & $\begin{array}{l}\text { Deviance } \\
\text { explained }\end{array}$ & AIC & $\begin{array}{c}\text { Residual } \\
\text { Df }\end{array}$ & $\begin{array}{c}\text { Df } \\
F \text {-test }\end{array}$ & $\begin{array}{c}\text { Deviance } \\
F \text {-test }\end{array}$ & $\boldsymbol{F}$ & $p$-value \\
\hline \multirow[t]{4}{*}{ HC } & $\bmod 1$ & 95.3 & 97 & -313.2 & 98.6 & NA & NA & NA & NA \\
\hline & $\bmod 2$ & 61.4 & 64 & -16.8 & 146 & 47.44 & 6.54 & 23.45 & $9.93 e^{-37}$ \\
\hline & $\bmod 3$ & 28.8 & 33 & 77.9 & 146.8 & 48.24 & 12.62 & 44.47 & $2.35 \mathrm{e}^{-49}$ \\
\hline & $\bmod 4$ & 62 & 64 & -20 & 146.9 & 48.36 & 6.48 & 22.78 & $2.43 \mathrm{e}^{-36}$ \\
\hline \multirow[t]{4}{*}{ TF } & $\bmod 1$ & 65.6 & 76 & -24.6 & 109.3 & NA & NA & NA & NA \\
\hline & $\bmod 2$ & 60 & 64 & -24.8 & 141.1 & 31.82 & 2.12 & 1.72 & 0.02 \\
\hline & $\bmod 3$ & 40.5 & 46 & 36.5 & 141.9 & 32.64 & 5.28 & 4.18 & $9.86 \mathrm{e}^{-09}$ \\
\hline & $\bmod 4$ & 49.3 & 53 & 8.4 & 145.2 & 35.95 & 4.06 & 2.91 & $1.03 \mathrm{e}^{-05}$ \\
\hline \multirow[t]{4}{*}{ BCM } & $\bmod 1$ & 76.3 & 83 & 157.9 & 109.4 & NA & NA & NA & NA \\
\hline & $\bmod 2$ & 63.9 & 67 & 199 & 142.1 & 32.67 & 13.36 & 3.28 & $1.92 \mathrm{e}^{-06}$ \\
\hline & $\bmod 3$ & 49.9 & 52 & 244.6 & 148 & 38.61 & 25.4 & 5.27 & $3.78 \mathrm{e}^{-12}$ \\
\hline & $\bmod 4$ & 55.5 & 58 & 228.3 & 145.8 & 36.36 & 20.53 & 4.53 & $4.97 \mathrm{e}^{-10}$ \\
\hline \multirow[t]{4}{*}{ MA } & $\bmod 1$ & 85.4 & 90 & 146.5 & 100.9 & NA & NA & NA & NA \\
\hline & $\bmod 2$ & 47.1 & 50 & 314.3 & 146.1 & 45.17 & 48.17 & 9.46 & $7.91 \mathrm{e}^{-21}$ \\
\hline & $\bmod 3$ & 52.4 & 57 & 303.9 & 139.1 & 38.17 & 39.55 & 9.19 & $2.70 \mathrm{e}^{-19}$ \\
\hline & $\bmod 4$ & 52.3 & 54 & 296.4 & 148 & 47.08 & 43.03 & 8.11 & $1.15 \mathrm{e}^{-18}$ \\
\hline \multirow[t]{4}{*}{ SP } & $\bmod 1$ & 37.1 & 50 & 249.7 & 122.3 & NA & NA & NA & NA \\
\hline & $\bmod 2$ & 9.9 & 14 & 285 & 147.1 & 24.78 & 20.99 & 3.57 & $1.48 \mathrm{e}^{-06}$ \\
\hline & $\bmod 3$ & 27.5 & 31 & 251.1 & 147 & 24.67 & 11.15 & 1.91 & 0.01 \\
\hline & $\bmod 4$ & 29.7 & 33 & 246.3 & 147 & 24.68 & 9.94 & 1.7 & 0.03 \\
\hline \multirow[t]{4}{*}{ CCA } & $\bmod 1$ & 61.1 & 72 & 212.2 & 112.1 & NA & NA & NA & NA \\
\hline & $\bmod 2$ & 45.7 & 50 & 241.3 & 142.1 & 29.99 & 15.44 & 2.88 & $2.99 \mathrm{e}^{-05}$ \\
\hline & $\bmod 3$ & 39.3 & 44 & 258 & 142.8 & 30.71 & 19.81 & 3.61 & $3.58 \mathrm{e}^{-07}$ \\
\hline & $\bmod 4$ & 45.2 & 48 & 239.5 & 145.8 & -33.7 & -16.71 & 2.78 & $3.10 \mathrm{e}^{-05}$ \\
\hline
\end{tabular}


40 years of benthic community change on the reefs of Curaçao and Bonaire

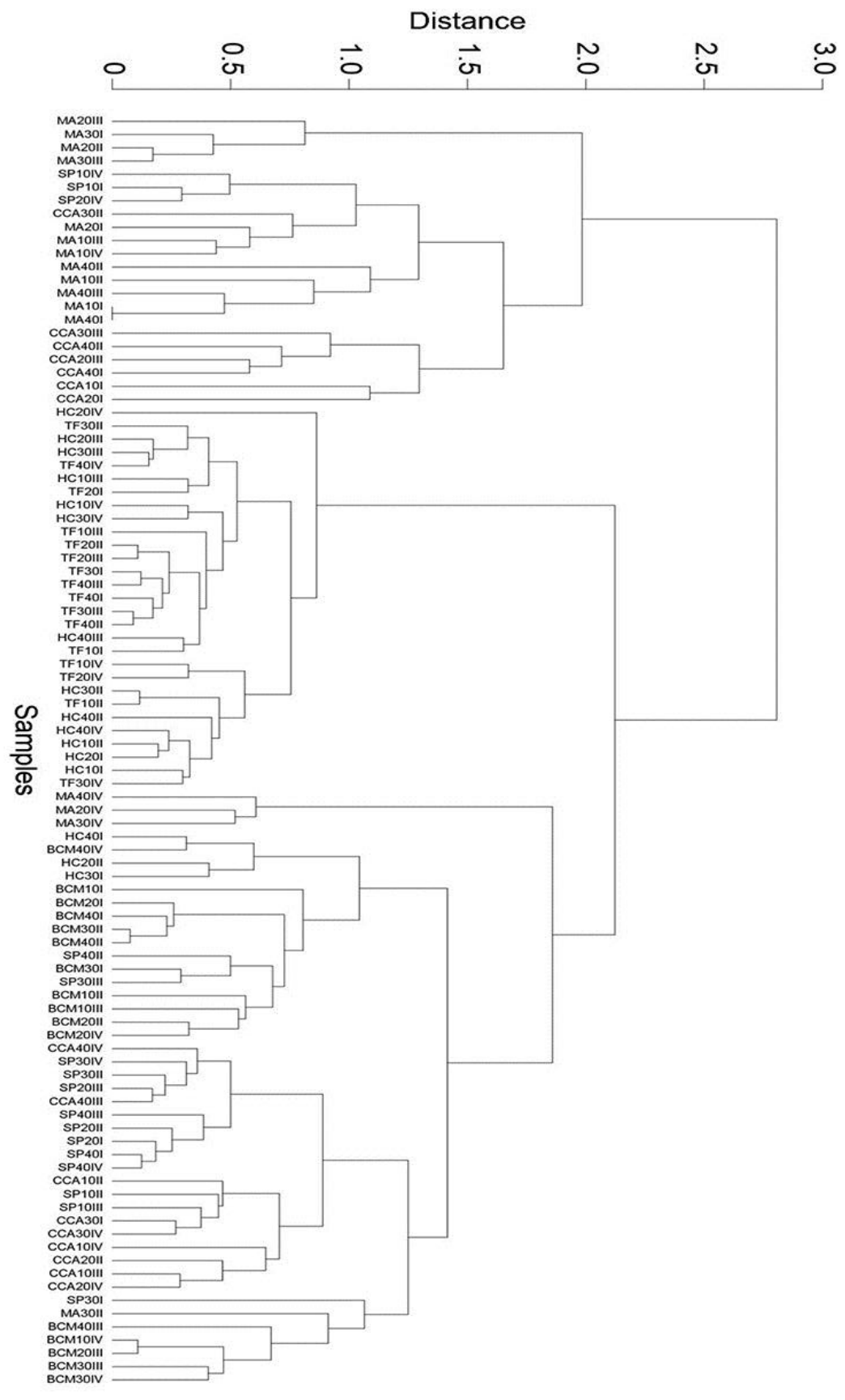

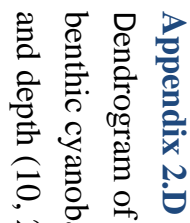

绕 号

w.

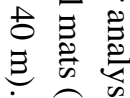

कิ

ก

है

今

बĩ

ह

3

is

है

突

टิ

ڤ̊ำ

32

क्ष

ते है

@

Бे

$\stackrel{2}{0}$

है

ลิ

$\Omega$

$\cong$

\%

है ข

约

影䁁 
Appendix 2.E

Community seriation and correlation indices. The index of multivariate seriation for each individual quadrat $(P)$ and rank correlations in multivariate community development between sites at equal depth $(P 2)$. Values significant at the .05 level are given in italic and at the .01 level in bold. $p$-values in parentheses.

\begin{tabular}{|c|c|c|c|c|}
\hline Depth (m) & $\begin{array}{c}\text { Within } \\
\text { Sites }\end{array}$ & $P$ & $\begin{array}{c}\text { Between } \\
\text { Sites }\end{array}$ & $P_{2}$ \\
\hline \multirow[t]{6}{*}{10} & I & $\mathbf{0 . 5 3}(.002)$ & I x II & $\mathbf{0 . 5 7}(.002)$ \\
\hline & II & $\mathbf{0 . 5 6}(.002)$ & I x III & $\mathbf{0 . 4 7}(.009)$ \\
\hline & III & $\mathbf{0 . 6 8}(.001)$ & I x IV & $\mathbf{0 . 5 8}(.001)$ \\
\hline & IV & $0.66(.003)$ & II x III & $0.24(.10)$ \\
\hline & & & II x IV & $0.19(.15)$ \\
\hline & & & III x IV & $\mathbf{0 . 7 4}(.004)$ \\
\hline \multirow[t]{6}{*}{20} & I & $\mathbf{0 . 7 8}(.001)$ & I x II & $\mathbf{0 . 5 9}(.001)$ \\
\hline & II & $\mathbf{0 . 8 7}(.001)$ & I x III & $\mathbf{0 . 6 4}(.004)$ \\
\hline & III & $\mathbf{0 . 8 3}(.001)$ & I x IV & $\mathbf{0 . 5 2}(.007)$ \\
\hline & IV & $0.76(.001)$ & II x III & $\mathbf{0 . 7 1}(.001)$ \\
\hline & & & II x IV & $\mathbf{0 . 8 0}(.001)$ \\
\hline & & & III x IV & $\mathbf{0 . 7 6}(.001)$ \\
\hline \multirow[t]{6}{*}{30} & I & $\mathbf{0 . 8 2}(.001)$ & I x II & $\mathbf{0 . 6 5}(.002)$ \\
\hline & II & $\mathbf{0 . 5 8}(.004)$ & I x III & $\mathbf{0 . 6 4}(.004)$ \\
\hline & III & $\mathbf{0 . 8 3}(.001)$ & I x IV & $\mathbf{0 . 5 7}(.004)$ \\
\hline & IV & $\mathbf{0 . 6 4}(.003)$ & II x III & $\mathbf{0 . 6 4}(.001)$ \\
\hline & & & II x IV & $0.30(.049)$ \\
\hline & & & III x IV & $\mathbf{0 . 6 1}(.001)$ \\
\hline \multirow[t]{6}{*}{40} & I & $\mathbf{0 . 5 2}(.001)$ & I x II & $0.53(.025)$ \\
\hline & II & $0.39(.010)$ & I x III & $0.47(.030)$ \\
\hline & III & $0.36(.025)$ & I x IV & $0.34(.13)$ \\
\hline & IV & $\mathbf{0 . 5 6}(.009)$ & II x III & $\mathbf{0 . 5 4}(.010)$ \\
\hline & & & II x IV & $0.39(.087)$ \\
\hline & & & III x IV & $0.06(.33)$ \\
\hline
\end{tabular}




\section{Appendix 2.F}

Pairwise correlations $(P)$ of community development between depths within each site. Values significant at the .05 level are given in italic and at the .01 level in bold. $p$-values in parentheses.

\begin{tabular}{c|c|c|c|c|c} 
Site & $\begin{array}{c}\text { Between } \\
\text { Depths }\end{array}$ & $\boldsymbol{P}$ & Site & $\begin{array}{c}\text { Between } \\
\text { Depths }\end{array}$ & $\boldsymbol{P}$ \\
\hline I & $10 \times 20$ & $0.31(.064)$ & III & $10 \times 20$ & $\mathbf{0 . 7 5}(.001)$ \\
\hline & $10 \times 30$ & $0.35(.044)$ & & $10 \times 30$ & $\mathbf{0 . 5 1}(.007)$ \\
\hline & $10 \times 40$ & $0.41(.074)$ & & $10 \times 40$ & $0.15(.17)$ \\
\hline & $20 \times 30$ & $0.41(.018)$ & & $20 \times 30$ & $\mathbf{0 . 6 7}(.001)$ \\
\hline & $20 \times 40$ & $0.38(.030)$ & & $20 \times 40$ & $0.30(.058)$ \\
\hline & $30 \times 40$ & $0.13(.25)$ & & $30 \times 40$ & $0.32(.056)$ \\
\hline II & $10 \times 20$ & $0.44(.015)$ & IV & $10 \times 20$ & $\mathbf{0 . 6 4}(.004)$ \\
\hline & $10 \times 30$ & $0.37(.022)$ & & $10 \times 30$ & $\mathbf{0 . 6 9}(.006)$ \\
\hline & $10 \times 40$ & $0.31(.038)$ & & $10 \times 40$ & $\mathbf{0 . 8 2}(.002)$ \\
\hline & $20 \times 30$ & $\mathbf{0 . 5 3}(.007)$ & & $20 \times 30$ & $\mathbf{0 . 7 1}(.002)$ \\
\hline & $20 \times 40$ & $0.23(.18)$ & & $20 \times 40$ & $0.73(.011)$ \\
\hline & $30 \times 40$ & $\mathbf{0 . 6 1}(.001)$ & & $30 \times 40$ & $\mathbf{0 . 7 8}(.001)$ \\
\hline
\end{tabular}

\section{Appendix 2.G}

Close up of site IV (20 m) in 1989 showing Trididemnum solidum covering A) large portion of the reef; B) Benthic cyanobacterial mats covering most of the substratum in site I (20 m) in 2013.
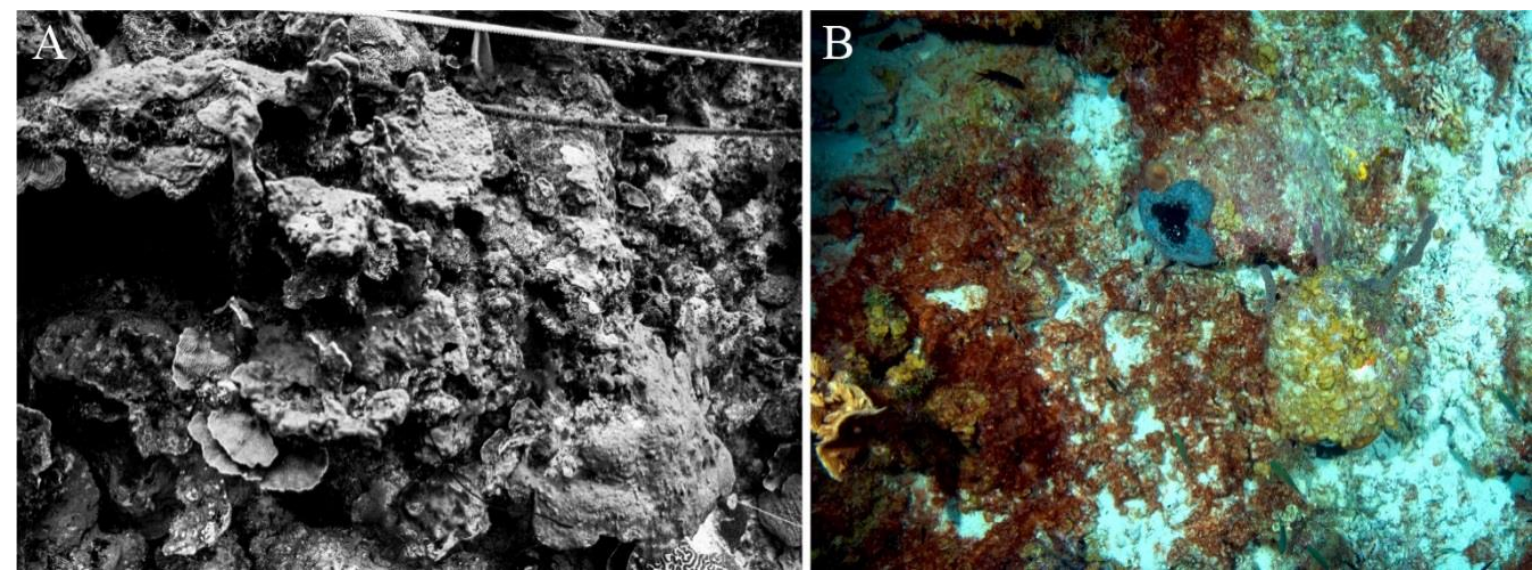


\section{Appendix 2.H}

Summary of the GAM model used for each of the six main benthic groups at a certain site and depth. Including estimated degrees of freedom (edf) and $p$-value of the non-linear trends.

\begin{tabular}{c|c|c|c|c|c|c|c} 
Site & Depth & \multicolumn{2}{c}{ Hard coral } & \multicolumn{2}{c}{ Algal turf } & \multicolumn{2}{c}{ BCMs } \\
\hline & & edf & $p$-value & edf & $p$-value & edf & $p$-value \\
\hline I & 10 & 5 & $<0.001$ & 1 & 0.589 & 2.8 & $<0.001$ \\
\hline & 20 & 3.4 & $<0.001$ & 1.8 & 0.107 & 1 & 0.002 \\
\hline & 30 & 4 & $<0.001$ & 1.9 & 0.001 & 1 & $<0.001$ \\
\hline & 40 & 3.9 & $<0.001$ & 1.8 & $<0.001$ & 1 & 0.195 \\
\hline II & 10 & 2.1 & $<0.001$ & 1 & & & \\
\hline & 20 & 1.5 & $<0.001$ & 2.3 & $<0.001$ & 1.9 & $<0.001$ \\
\hline & 30 & 1 & 0.256 & 2 & $<0.001$ & 1 & 0.017 \\
\hline & 40 & 5 & $<0.001$ & 2.3 & $<0.001$ & 1 & 0.03 \\
\hline & & & & & & & $<$ \\
\hline III & 10 & 1.9 & $<0.001$ & 2.6 & 0.038 & 4 & $<0.001$ \\
\hline & 20 & 1 & $<0.001$ & 2.4 & $<0.001$ & 1.3 & $<0.001$ \\
\hline & 30 & 2.4 & $<0.001$ & 2.3 & $<0.001$ & 1 & $<0.001$ \\
\hline & 40 & 2.8 & $<0.001$ & 1.8 & 0.014 & 3.8 & $<0.001$ \\
\hline & & & & & & & $<$ \\
\hline
\end{tabular}

\begin{tabular}{c|c|c|c|c|c|c|c} 
Site & \multicolumn{1}{|c}{ Depth } & \multicolumn{2}{c}{ Macroalgae } & \multicolumn{2}{c}{ Sponges } & CCA \\
\hline & & edf & p-value & edf & p-value & edf & p-value \\
\hline I & 10 & 1 & 1 & 1 & 0.082 & 1 & 0.006 \\
\hline & 20 & 1.9 & $<0.001$ & 1 & 0.244 & 1.4 & $<0.001$ \\
\hline & 30 & 3.4 & $<0.001$ & 1.3 & 0.048 & 1.5 & $<0.001$ \\
\hline & 40 & 1 & 1 & 1 & 0.026 & 2.2 & 0.041 \\
\hline II & 10 & 1 & 0.752 & 1.4 & 0.252 & 1 & $<0.001$ \\
\hline & 20 & 3.3 & $<0.001$ & 1 & 0.005 & 2.6 & 0.005 \\
\hline & 30 & 4 & $<0.001$ & 1 & 0.837 & 2.1 & 0.044 \\
\hline & 40 & 1 & 0.691 & 1 & 0.336 & 2.3 & 0.009 \\
\hline III & 10 & 2.1 & $<0.001$ & 1 & 0.014 & 2 & 0.01 \\
\hline & 20 & 2.1 & $<0.001$ & 1.2 & 0.49 & 2.2 & $<0.001$ \\
\hline & 30 & 3.3 & $<0.001$ & 1 & 0.023 & 2.4 & 0.005 \\
\hline & 40 & 2.3 & 0.039 & 1.3 & 0.569 & 1 & 0.888 \\
\hline & & & & & & & \\
\hline
\end{tabular}




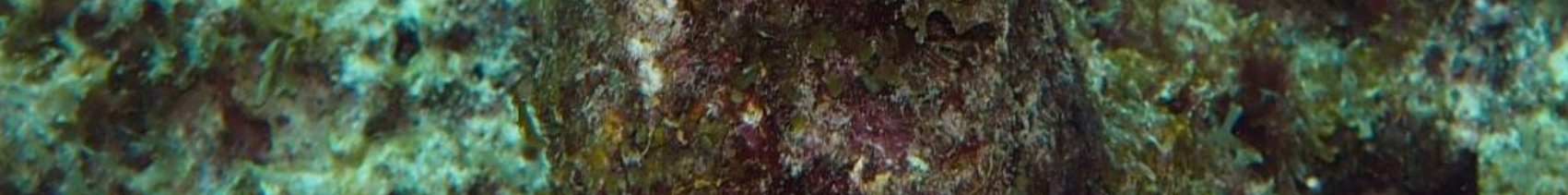

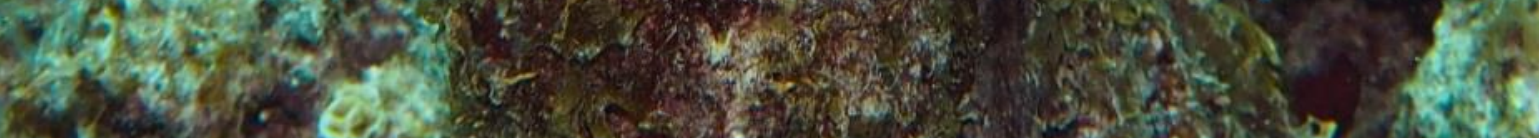

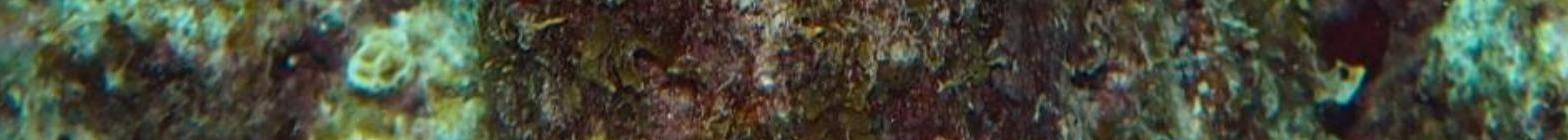

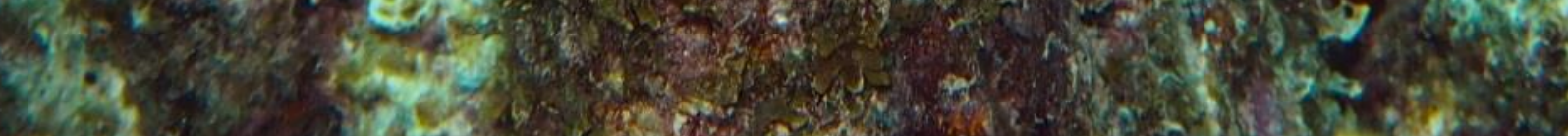

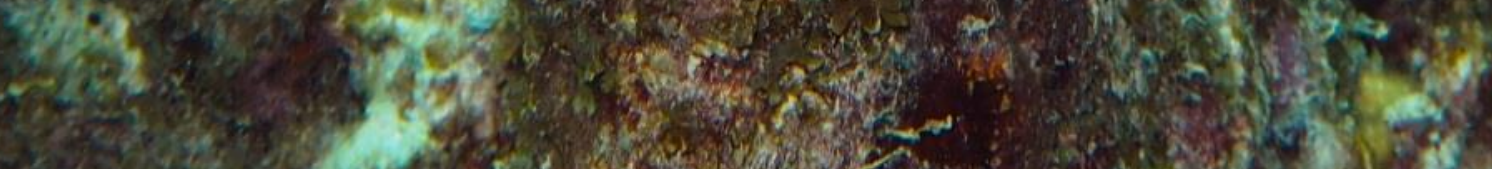

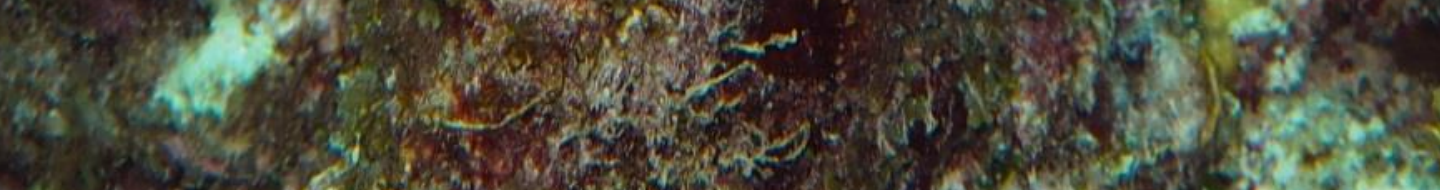

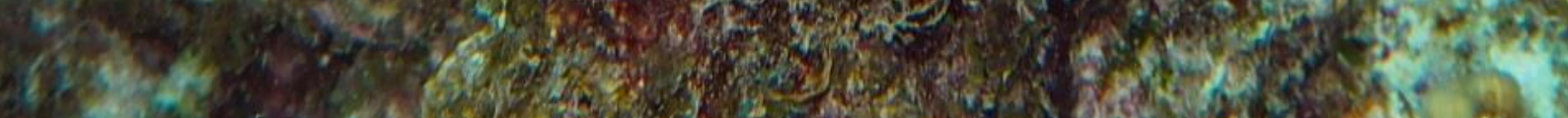




\title{
CHAPTER III \\ Long-term shifts in coral communities on shallow to deep reef slopes of Curaçao and Bonaire: are there any winners?
}

\author{
Didier M. De Bakker, Erik H. Meesters, Rolf P. M. Bak, Gerard Nieuwland and \\ Fleur C. Van Duyl
}

\section{Abstract}

Tropical coral reefs are among the most biologically diverse and economically important ecosystems on earth. Nevertheless, we found dramatic changes in coral communities on the reef slopes of Curaçao and Bonaire since 1973. Cover and abundance declined for virtually all coral species. The data show a shift from communities dominated by framework building species (e.g., Orbicella spp.) to communities consisting of small opportunistic, phenotypically plastic, species, including few remaining structural colonies. Madracis mirabilis, Porites astreoides, Pseudodiploria strigosa, and Agaricia lamarcki are at present modest 'winners' in the coral assemblage, although overall cover declined also for these species. Increased frequency and intensity of events inducing coral mortality and ongoing reduction in suitable hard substratum, provided by the remnants of large colony building species, could reduce the chance of these species to remain winners in the longer run. The observed loss in coral cover and the shift from larger structural to smaller opportunistic species reduced reef carbonate production by $67 \%$ and therewith, in combination with a trend toward smaller coral colonies, reef complexity. Alarmingly, reefs at upper-mesophotic depths $(30-40 \mathrm{~m})$ did not escape the general degradation of the coral community. The negative effects are larger around densely populated areas where local stressors are adding to degradation caused, for instance, by region wide mass bleaching. Without proper conservation and management this already dramatic degradation will continue and turn more and more coral species into 'losers'. 


\section{Introduction}

Tropical reefs are among the most biologically rich ecosystems on earth providing income for millions of people through fisheries, tourism and coastal protection (Moberg and Rönnbäck 2003). On tropical reefs scleractinian corals are largely responsible for the architectural complexity due to the deposition of calcium carbonate and thereby provide habitat, food, and refuge for many taxonomic groups. Over the past decades corals reefs have been degrading worldwide at unprecedented rates (Gardner et al. 2003; Bak et al. 2005; Pandolfi and Jackson 2006). Deteriorating coastal water quality (Rogers 1990; Williams et al. 2002; Vega-Thurber et al. 2014), overexploitation of herbivores (Jackson et al. 2001) and anthropogenic induced climate change (Glynn 1996; Aronson et al. 2002a; Wilkinson and Souter 2008) are generally associated with this ubiquitous decline in coral cover (Jackson et al. 2014). These stressors negatively impact coral resilience, impeding the ability of corals to cope with disease, bleaching or physical disturbance, which in turn affects their competitive position with respect to other reef organisms such as macroalgae or sponges (Vega-Thurber et al. 2014; Zaneveld et al. 2016). Aforementioned developments are suggested to cause shifts in coral communities from assemblages dominated by framework building corals to a dominance of more persistent and opportunistic non-framework building species (e.g., Green et al. 2008; Alvarez-Filip et al. 2011a; Darling et al. 2012; Perry et al. 2015a).

Whether coral species are likely to become winners or losers in the changing reef environment depends strongly on their specific life-history traits (Knowlton 2001; Van Woesik et al. 2011). In an attempt to predict what species are likely to be lost and what species may be more persistent, several studies have divided species over groups based on shared characteristics such as: way of propagation, growth rate and thermo-tolerance (e.g., Edinger and Risk 2000; Murdoch 2007). Recently, Darling et al. (2012) divided 147 coral species from tropical reefs all over the world over four main groups: competitive, weedy or ruderal, stress-tolerant and generalist. This grouping was based on 11 functional traits. In the early 1970s coral reefs were still generally dominated by stress-tolerant, competitive and generalist species. It is hypothesized, however, that coral communities are shifting toward species assemblages comprising mainly of phenotypically plastic weedy species and some persistent framework building (stress-tolerant, generalist) species (Darling et al. 2012; Grottoli et al. 2014; McClanahan et al. 2014; Perry et al. 2015a). Since the frequency and intensity of events inducing coral mortality (e.g., bleaching and hurricanes) is predicted to increase, recovery and adaptation will become progressively more difficult for all coral species (Hoegh-Guldberg 1999; Van Woesik et al. 2011; McClanahan et al. 2014). As a consequence, the contribution of corals in benthic reef communities will likely decrease even further and the most stress susceptible coral species may disappear entirely at many reefs (Knowlton 2001; HoeghGuldberg et al. 2007). Predicting trajectories of coral community change, however, is extremely complex due to the numerous factors involved (Hughes and Tanner 2000; Done et al. 2010). Additionally, shifts in coral communities may occur slowly and are therefore often difficult to distinguish (Pauly 1995). There appears to be a consistent lack of long-term temporal quantitative data documenting the trajectories of different species in the coral community. 
Here, we provide data on the coral community dynamics at reefs on the southern Caribbean islands of Curaçao and Bonaire. Over the course of the studied time series human populations in the Caribbean increased from 27 million in 1973 to approximately 43 million in 2015 (United Nations, Department of Economic and Social Affairs, Population, Division, 2015). As a consequence, the Caribbean basin experiences chronic decline of water quality and overexploitation of fish (Siung-Chang 1997; Jackson et al. 2014). Due to climate change and increasing sea water temperature, Caribbean corals have been exposed to mass bleaching events in 1998 (Aronson et al. 2002a), 2005 (Wilkinson and Souter 2008; Eakin et al. 2010) and 2010 (Alemu and Clement 2014). Furthermore, White Band Disease greatly reduced the cover of Acropora spp. from the late seventies to the mid-eighties, which negatively impacted rates of calcification and architectural complexity of the shallow reef (Gladfelter 1982; Aronson and Precht 2001). Additionally, herbivory was greatly reduced due to the mass-mortality of the Diadema antillarum sea-urchin in 1983 (Bak et al. 1984), formerly important in controlling the densities of competitive algae (De Ruyter Van Steveninck and Bak 1986).

In comparison to other tropical regions (e.g., the Pacific), the Caribbean has relatively few coral species (Veron 2014). As a consequence, certain ecologically relevant traits of specific species may be lost more rapidly in the Caribbean compared to other regions (Nyström 2006). In many Caribbean sites the cover of corals providing complex 3D reef structure, mostly Orbicella spp. (= Montastraea, sensu Budd et al. 2012) and Acropora spp. was already strongly reduced over the past decades (Van Duyl 1985; Aronson and Precht 2001; Bruckner and Bruckner 2006). The calcification balance on many of these reefs may currently have shifted toward net erosion which will result in the reduction of architectural complexity (Alvarez-Filip et al. 2009; Kennedy et al. 2013; Perry et al. 2013). This may drastically alter reef functioning and lead to loss of biodiversity as well as a reduction in important ecosystem services for coastal human population such as wave protection and food security (Moberg and Rönnbäck 2003; AlvarezFilip et al. 2009, 2011b; Graham and Nash 2013; Newman et al. 2015).

On degrading Caribbean reefs, phenotypically plastic corals such as Madracis spp., Porites spp., Siderastrea spp. or Agaricia spp., are expected to replace species controlling reef structure like Acropora spp. and Orbicella spp. (Pandolfi and Jackson 2006) and become dominant in coral communities (Jackson 2001; Aronson et al. 2002b; Green et al. 2008; Perry et al. 2015a). These species are, however, often small and generally not capable of fulfilling the important ecological functions provided by large framework building species (Bellwood et al. 2004; Nyström 2006; Alvarez-Filip et al. 2013; Perry et al. 2013). Moreover, it may be expected that in the long run opportunistic weedy species (Darling et al. 2012) will also disappear since no coral species appears to be effectively insensitive to anthropogenic impact (McClanahan et al. 2014). We aim, on the basis of images from 18 time series collected at five reef sites in Bonaire and Curaçao, and covering up to 40 years, to (1) determine temporal and spatial changes in dominant coral species, (2) link observed trajectories of the coral community to the life-history strategies of large framework building species and opportunistic weedy species, (3) estimate the relative loss 
of carbonate production due to changes in coral species composition and cover over time, and finally (4) discriminate between the 'winners' and 'losers' on these Caribbean coral reefs.

\section{Materials and Methods}

\section{Site Description and Procedure for Image Collection}

The spatio-temporal dynamics of coral reef communities at four southern Caribbean sites were tracked based on photographic records spanning a period of more than 40 years (Bak and Nieuwland 1995; Bak et al. 2005). The survey sites are located at the leeward side of the islands of Curaçao: Carmabi Buoy One (sites I and II) and Carmabi Buoy Two (site III); and Bonaire: Karpata (site IV) (Figure 3.1 \& Table 3.1). The leeward fringing reef on both islands is characterized by a shallow reef terrace that transitions into a seaward slope with a drop off that varies from less than $20^{\circ}$ to vertical (Bak 1975; Van Duyl 1985). At each site four permanent quadrats $\left(9 \mathrm{~m}^{2}\right)$ were set out and marked in 1973 on the fore-reef slope at 10, 20, 30, and $40 \mathrm{~m}$ depth, one quadrat at each depth. The $10 \mathrm{~m}$ quadrats were too deep to cover the Acropora palmata and Acropora cervicornis zones, which are both situated, if present, on the shallow reef terrace at less than $10 \mathrm{~m}$ depth (Bak 1977). Surveys were conducted with variable intervals between 1973 and 2014 and comprised of collecting overview images complemented by a series of detail recordings. In addition to the four main sites a fifth site, Awa Blancu (AB), located at the far south-eastern side of Curaçao, was included with a quadrat positioned at $10 \mathrm{~m}$ (since 1983 ) and $20 \mathrm{~m}$ (since 1992) depth. AB is located relatively far upstream of any urban areas and therefore suffers relatively less local anthropogenic disturbance (Gast et al. 1999; Lapointe and Mallin 2011) (Figure 3.1). This series was used as a relatively pristine reference to the shallow fore-reef slope (10-20 m) of sites I-IV which lie downstream of densely populated areas and close to outlet bays (Van Duyl 1985). Per series, a maximum of 9 years was analysed (Table 3.1).

\section{Coral Community Characteristics}

All coral colonies that were present in a quadrat were recorded. A coral was considered an individual colony when the tissue was distinctly separated in space from the tissue of any neighbouring corals. When a colony was located on the edge of the quadrat only the area within the actual $9 \mathrm{~m}^{2}$ quadrat was included in the survey. Fitted image overlays including all coral colonies were created in Photoshop CC 2015 (Adobe) and Scion Image v4.0.3.2. Actual live surface area $\left(\mathrm{cm}^{2}\right)$ and colony outline $(\mathrm{cm})$ were extracted from the overlay images and used as general metrics for coral colony size. The ratio between colony outline and live surface area of each colony was used as a measure for colony shape (shape index). Larger round colonies have a relatively low shape index. Partial mortality, particularly in larger colonies (Type II, sensu Meesters et al. 1996), or exposure to competing organisms leads to a relatively larger outline compared to the surface area, resulting in a higher shape index. Colonies were identified to species level, but because the different Orbicella species (O. annularis, O. faveolata, $O$. franksi) could not be separated reliably in retrospect, they were grouped into the Orbicella complex. For the same reason there was no further distinction made between the species Madracis pharensis, M. formosa, M. senaria, and M. decactis, which were grouped together under the Madracis 
taxonomic spp. complex. As a base-line, the classification system proposed by Darling et al. (2012) was used to a priori subdivide the coral species. The class "stress-tolerant," however, proved at times confusing and was replaced by "large framework building." This class included the entire Orbicella species complex, in contrast to Darling et al. (2012) who categorized $O$. faveolata and $O$. franksi as "generalists." "Weedy" species are here referred to as opportunistic.

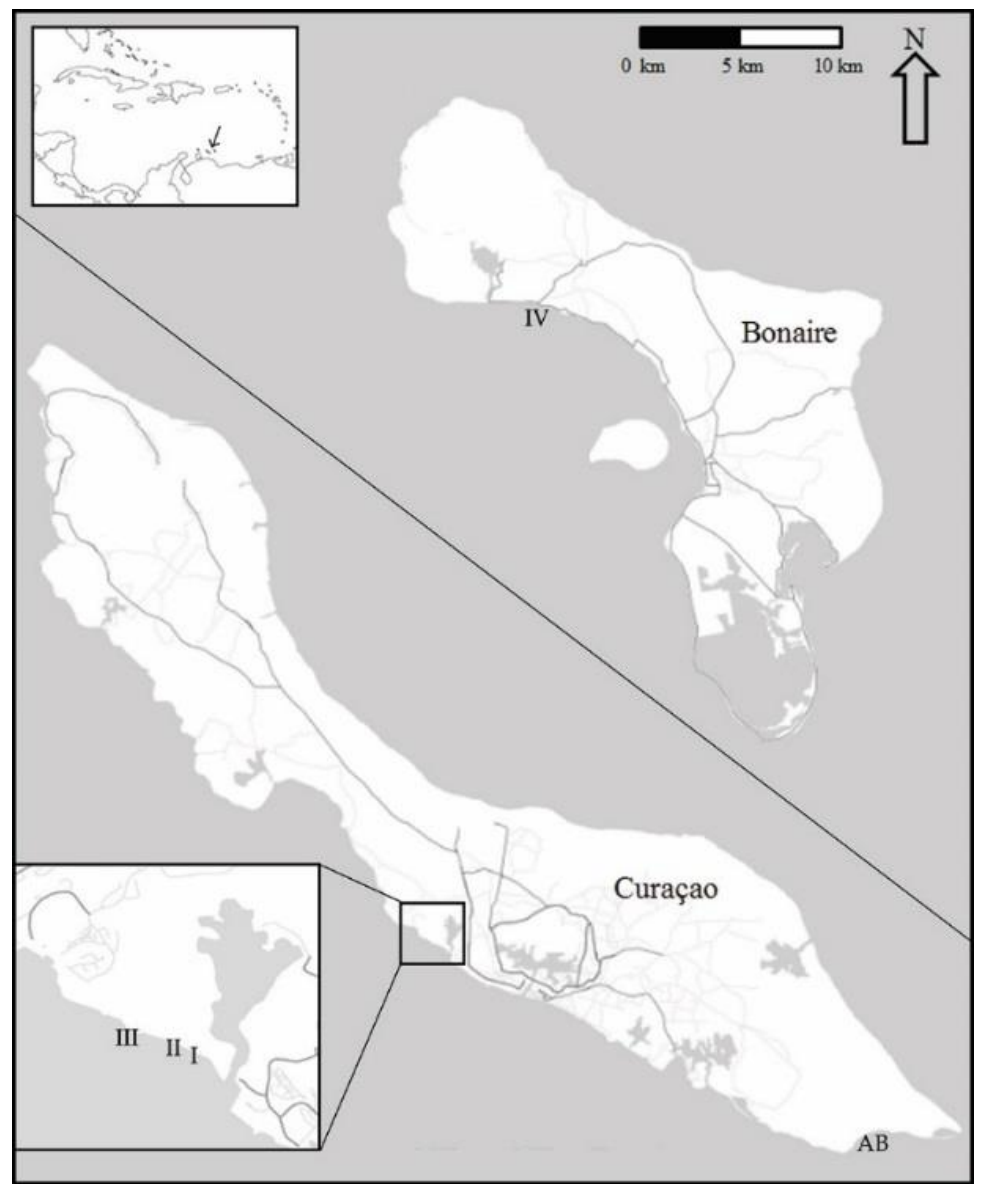

Figure 3.1. Quadrat locations at Curaçao (I-III, AB) and Bonaire (IV). Including their position in the wider Caribbean sea (top left). Enlarged section at the lower left corner shows in detail the position of sites I-III on Curaçao.

\section{Statistical Analyses}

The original data consists of a matrix where each row represents a single coral colony in a certain quadrat at a certain year and columns display surface area, outline, shape and specific site characteristics of each colony. This set was used to acquire the total number of colonies in each quadrat. Subsequently, the total planar cover per species was calculated for each quadrat and expressed as a percentage of the total quadrat area $\left(9 \mathrm{~m}^{2}\right)$. Before statistical multivariate analysis 
the influence of less dominant species was increased by applying a square-root transformation. Non-metric Multi-Dimensional Scaling (nMDS) was used as a tool to display community development over time at each depth averaged over sites I-IV and also for AB at 10 and $20 \mathrm{~m}$. To test for a directional development of the community composition over time a seriation routine (Clarke and Warwick 1994) was conducted in the multivariate software program Primere v7.0.9 (RELATE function with $\mathrm{M}=9999$ simulations). This procedure allows testing of similarity of a community distance (sub)matrix to a model matrix based on the Spearman rank correlation coefficient (Kendall 1948; Clarke and Warwick 1994) and provides a measure (Rho) for linearity in temporal community development. A Rho-value close to 1 is indicative of a linear development over time. Similarly, a second stage routine was applied to submatrices of specific sites per depth to reveal consistencies or variations in temporal patterns of development in community composition among sites (Clarke et al. 2006). A positive correlation in the pattern of development of two time series will result in a high $R$ ho-value.

Table 3.1. Survey site characteristics. All years a site was visited (Years) and depths (m) at which permanent quadrats were positioned. Years visited are shown as the last two numbers of the year. Sites I-IV were first visited in 1973. The time series at Awa Blancu $10 \mathrm{~m}$ and $20 \mathrm{~m}$ have a different start, namely 1983 and 1992, respectively.

\begin{tabular}{|c|c|c|c|c|c|c|}
\hline Island & Site & Code & Longitude & Latitude & Years & Depth (m) \\
\hline \multirow[t]{5}{*}{ Curaçao } & Buoy One & I & $-68^{\circ} 97^{\prime} 532.0^{\prime \prime} \mathrm{W}$ & $12^{\circ} 12^{\prime} 585.0^{\prime \prime} \mathrm{N}$ & $\begin{array}{c}73 ; 75 ; 83 ; 92 ; 97 ; \\
02 ; 08 ; 12 ; 14\end{array}$ & $10 ; 20 ; 30 ; 40$ \\
\hline & & II & $-68^{\circ} 97^{\prime} 652.0^{\prime \prime} \mathrm{W}$ & $12^{\circ} 12^{\prime} 655.0^{\prime \prime} \mathrm{N}$ & $\begin{array}{c}73 ; 75 ; 83 ; 92 ; 97 ; \\
02 ; 08 ; 12 ; 14\end{array}$ & $10 ; 20 ; 30 ; 40$ \\
\hline & Buoy Two & III & $-68^{\circ} 97^{\prime} 778.3^{\prime \prime W}$ & $12^{\circ} 12^{\prime} 723.4^{\prime \prime} \mathrm{N}$ & $\begin{array}{c}73 ; 75 ; 83 ; 92 ; 97 \\
02 ; 08 ; 12 ; 14\end{array}$ & $10 ; 20 ; 30 ; 40$ \\
\hline & Awa Blancu & $\mathrm{AB}$ & $-68^{\circ} 78^{\prime} 381.2^{\prime \prime} \mathrm{W}$ & $12^{\circ} 04^{\prime} 029.9^{\prime \prime} \mathrm{N}$ & $83 ; 92 ; 08 ; 12 ; 14$ & 10 \\
\hline & & & & & $92 ; 02 ; 08 ; 12 ; 14$ & 20 \\
\hline Bonaire & Karpata & IV & $-68^{\circ} 34^{\prime} 413.2^{\prime \prime} \mathrm{W}$ & $12^{\circ} 20^{\prime} 998.0^{\prime \prime} \mathrm{N}$ & $\begin{array}{c}73 ; 75 ; 83 ; 92 ; 97 ; \\
02 ; 08 ; 12 ; 14\end{array}$ & $10 ; 20 ; 30 ; 40$ \\
\hline
\end{tabular}

Temporal variation in colony size distributions were determined based on univariate measures of colony size, namely, mean size, skewness and kurtosis (Mardia 1970), because changes in size frequency can be related to shifts in coral communities (Bak and Meesters 1998). The difference in mean colony size at sites I-IV over time (of all colonies) was assessed by two-way ANOVA for each depth based on fourth-root transformed data, with year (start and end) and site as categorical variables. Assumptions for ANOVA were confirmed visually (Zuur et al. 2007). Subsequently Post Hoc (Tukey HSD with Bonferroni correction) tests were applied to compare colony size at the start and end in each individual quadrat. Sites were also compared 
with reference site AB using a different starting year (since 1983 and 1992 for 10 and $20 \mathrm{~m}$ resp.). Various diversity indices were computed for each quadrat and year: Shannon-Wiener diversity index (Shannon and Weaver 1949), Simpson's diversity index (Simpson 1949), Pielou's evenness (Pielou 1969) and total number of species. Statistical testing of other community characteristics (e.g., no. of colonies, no. of species, diversity indices, skewness, kurtosis) was inappropriate because this would result in only one replicate value per quadrat. With the exception of the RELATE and Second stage routines all analyses were performed in the R programming environment v3.2.3 (R Core Team 2014). Within $\mathrm{R}$ the following packages were used: vegan (Oksanen et al. 2016) and moments (Komsta and Novomestky 2015).

\section{Carbonate Production}

The relative contribution of the coral community to reef carbonate production in $\mathrm{kg} \mathrm{CaCO} 3 \mathrm{~m}^{-2}$ $\mathrm{y}^{-1}$ in a quadrat at each measured point in time was estimated based on total planar cover of each species. Species-specific or nearest equivalent species accretion rates were adapted from the ReefBudget method as proposed by Perry et al. (2012), who acquired the various rates from the available literature. Since our data on coral cover was extracted from 2D-images the obtained estimates will be an underestimation compared to the ReefBudget method where 3D surface is included.

\section{Results}

\section{Consistencies and Variability in Trajectories of Community Change}

The coral community at the four main sites I-IV has since 1973 developed in a constant unidirectional degrading way (Figure 3.2 \& Table 3.2), which is illustrated in the nMDS plot by the generally linear arrangement of points (years) along the y-axis (Figure 3.2). This suggests a directional trend in community development gradually moving away from (upwards in Figure 3.2) the initial community compositions at the start of the time series, irrespective of depth. The first axis indicates a clear effect of depth caused by strong variations in community composition across the depth gradient. The trajectories at greater depths (30 and $40 \mathrm{~m}$ ) were very similar (Figure 3.2). At AB, change in community development was less pronounced compared to sites I-IV (Figure 3.2 \& Table 3.2), particularly at $20 \mathrm{~m}$ depth. The line of $10 \mathrm{~m}$ at the reference site $\mathrm{AB}$ was arranged relatively close to the average for sites I-IV at $10 \mathrm{~m}$, suggesting similarity in community composition. At AB 10 m the community in 2014 resembled the community of 1997-2002 at sites I-IV, implying a time lag in reef degradation. A longer time lag was observed for 20 (Figure 3.2). Also, the community at AB $20 \mathrm{~m}$ appears to be more different from sites IIV (Figure 3.2). At $20 \mathrm{~m}$ (recorded since 1992) the high, albeit not significant, Rho-value $(0.926, p=0.087)$ indicated linear development in coral community composition, but AB covered a shorter time period and less surveys were included. High Rho-values and nonsignificant p-values appear to be contradicting, but are likely the result of the low number of time points. In such a case more emphasis may be put on the Rho-value (Clarke and Warwick 1994), but these values should be interpreted with caution. 
Overall, the total area covered by hard corals (Figure 3.3A) declined on average from $33.8 \%$ in 1973 to $10.1 \%$ in 2014 over the main sites (I-IV), resp. $95 \%$ confidence intervals: 25.7 - 42.9 and $6.9-14.5$. The observed decline was highly comparable among depths but was greatest at $20 \mathrm{~m}$ depth, showing a decrease of $78.9 \%$ from $43 \%$ in 1973 to $9.1 \%$ cover in 2014 (Figure 3A). In comparison coral cover at 10, 30, and $40 \mathrm{~m}$ depth in 1973 was 42, 33, and $23 \%$ and declined by $68.9,63.5$, and $58.6 \%$ respectively to 13,12 , and $9.5 \%$ cover in 2014 . This indicates a pronounced reduction in coral cover along the entire depth gradient studied. In comparison, coral cover at AB declined by $31 \%$ at $10 \mathrm{~m}$ and $49 \%$ at $20 \mathrm{~m}$ depth since 1983 and 1992, resp. (Figure 3.3A \& Table 3.3). The mean percentage of change in cover at these two depths at sites I-IV, over the same time-span as AB, was much larger with $71 \%(10 \mathrm{~m})$ and $76 \%(20 \mathrm{~m})$, resp. 95\% confidence intervals: $24-96$ and $73-78$.

Analogous with the decline in coral cover the total number of coral colonies decreased in the majority of the quadrats, particularly between 1973 and 1983 (Figure 3.3B \& Table 3.3). At 10 $\mathrm{m}$ depth the number of colonies initially decreased almost exponentially in the first decade, but subsequently the number stagnated and finally started increasing again after the late 1990s (Figure 3.3B). This pattern was largely driven by the dynamic M. mirabilis, which has been a dominant species at $10 \mathrm{~m}$ depth (Figure 3.4), but through time proved to be highly variable in the number of colonies and cover. By 2014, M. mirabilis had increased in density at sites III and IV, and also at AB (steep increase in Figure 4.3B), while an obvious decrease was observed at site I. After 1983 the number of colonies on mesophotic depths (30 and $40 \mathrm{~m}$ ) remained relatively constant until the late 1990s, after which a gradual decline was observed.

In addition to the decline in the number of colonies (Figure 3.3B), the community at sites I-IV has generally shifted to a relative dominance by smaller colonies in 2014 compared to 1973 (Figure 3.3C \& Table 3.3). At $10 \mathrm{~m}, 20 \mathrm{~m}$ and $40 \mathrm{~m}$ a significant difference was observed between years $[F=78, p<0.001, F=22, \mathrm{p}<0.001$ and $F=7, \mathrm{p}<0.01$, resp.] and sites $[F=$ 97, $p<0.001, F=17, p<0.001$ and $F=8, p<0.001$, resp.]. At $30 \mathrm{~m}$ a significant difference was only found between sites $[F=12, p<0.001]$. A Post Hoc test comparing sites including AB showed significant larger colonies at AB in 1992 at $20 \mathrm{~m}$ depth (all $p<0.001$ ), but by 2014 a dramatic decrease resulted in values that are comparable to those at sites I-IV (Figure 3.3C \& Table 3.3). At $10 \mathrm{~m}$ depth, only site I differed significantly from AB in 1983 and 2014 ( $p<$ 0.05). Reduced mean colony size over time is in part a consequence of the loss of large colony building species such as Orbicella spp. and a still relatively high number of small colonies of, for instance, M. mirabilis (Figure 3.5). Both the Orbicella complex (dominant on the shallow fore-reef) and $A$. lamarcki (dominant on the deep fore-reef) showed a shift toward smaller colonies (Figure 3.5). This shift is also shown by an increased positive skewness for many quadrats, including AB, in 2014 compared to the beginning of the time series (Table 3.3). In contrast to the two other dominant species, the opportunistic M. mirabilis does not show a clear shift in colony size, but more a general loss in the number of colonies (Figure 3.5). Coral colony "shape" (outline:area) appears to represent a shift from larger round colonies to smaller and more complexly shaped colonies (Figure 3.3D). This is likely a consequence of increased 
occurrence of partial colony mortality. Partial mortality may result in a relatively large increase in outline compared to a minor decrease in total surface area.

Table 3.2. Linearity of community development over time for each individual quadrat by depth. The degree of seriation (Rho) was based on the relative cover of all species present within a quadrat (I-IV and reference site AB). Strong linearity in development results in Rho-values close to 1. Significant Rho-values are given in bold, respective p-values in parentheses.

\begin{tabular}{|c|c|c|c|c|c|}
\hline Depth & Site & Rho (pvalue) & Depth & Site & Rho (pvalue) \\
\hline \multirow[t]{5}{*}{10} & I & $0.678(.001)$ & 30 & I & $0.936(.001)$ \\
\hline & II & $0.861(.001)$ & & II & $0.814(.001)$ \\
\hline & III & $0.891(.001)$ & & III & $0.792(.001)$ \\
\hline & IV & $0.911(.001)$ & & IV & $0.851(.001)$ \\
\hline & $\mathrm{AB}$ & $0.370(.328)$ & & & \\
\hline \multirow[t]{5}{*}{20} & I & $0.843(.001)$ & 40 & I & $0.710(.001)$ \\
\hline & II & $0.899(.001)$ & & II & $0.797(.001)$ \\
\hline & III & $0.688(.002)$ & & III & $0.910(.001)$ \\
\hline & IV & $0.905(.001)$ & & IV & $0.696(.002)$ \\
\hline & $\mathrm{AB}$ & $0.926(.087)$ & & & \\
\hline
\end{tabular}

\section{Trends in Species Composition}

Over the course of the period surveyed $(1973$ - 2014) a total of 30 scleractinian coral species were recorded in the quadrats. After combining the various Madracis and Orbicella species into two groups 24 species/complexes remained (Table 3.4). The number of species declined in the majority of the quadrats between 1973 and 2014 (Figure 3.3E \& Table 3.3). In 2014 opportunistic species accounted for $62 \%$ of the total coral cover, compared to $53 \%$ in 1973 at sites I-IV. At AB, framework building species kept dominating the coral community at both 10 and $20 \mathrm{~m}$, accounting for $52 \%$ and $85 \%$, respectively, of the total coral cover in 2014 (Appendix 3.C). The cover of competitive species (Acropora spp. and Dendrogyra cylindrus) in the quadrats was negligible. Highest coral diversity was generally found around $20 \mathrm{~m}$ depth and lowest at $40 \mathrm{~m}$ depth (Table 3.3, Figure 3.3E). A relatively low, but significant, correlation (Rho) was found among multiple sites, including AB, at the shallow (10-20 m) fore-reef (Appendix 3.A). This observation coincides with a clear variation in species composition and trajectories of coral community development between these sites, particularly regarding the dominance of M. mirabilis, Orbicella spp. or Montastraea cavernosa (Figure 3.4). In comparison, the species composition at 30 and $40 \mathrm{~m}$ depth was highly comparable among sites, with an overall dominance of Agaricia lamarcki (Figure 3.4), which resulted in strong correlations between all sites (Appendix 3.A). 
Figure 3.2. Coral community dynamics on Curaçao and Bonaire. Non-metric MultiDimensional Scaling ordinations (nMDS) displaying mean community development over time of sites I-IV, separated by depth (I-IV-10 m, I-IV-20 m, I-IV-30 m, I-IV-40 m) and the reference site Awa Blancu 10 and $20 \mathrm{~m}$ (AB-10 $\mathrm{m}$ and $A B-20 \mathrm{~m}$ ). Each colour-line type combination corresponds to a specific site-depth combination. Lines represent the degree and direction of community change between two surveys. All surveys are labelled as the last two numbers of the year a survey was conducted. The closer the distance between two points, the more similar in composition they are.

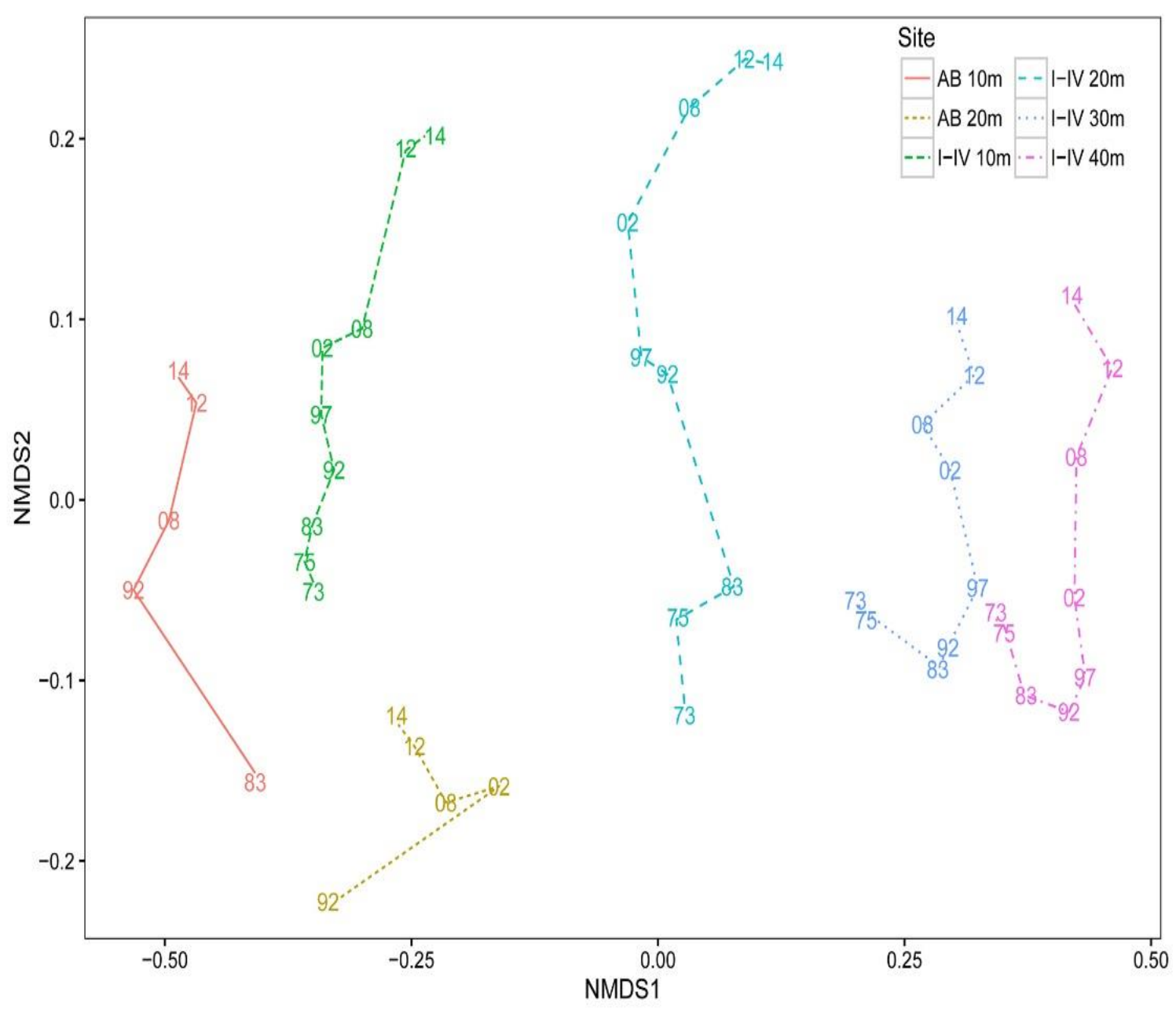



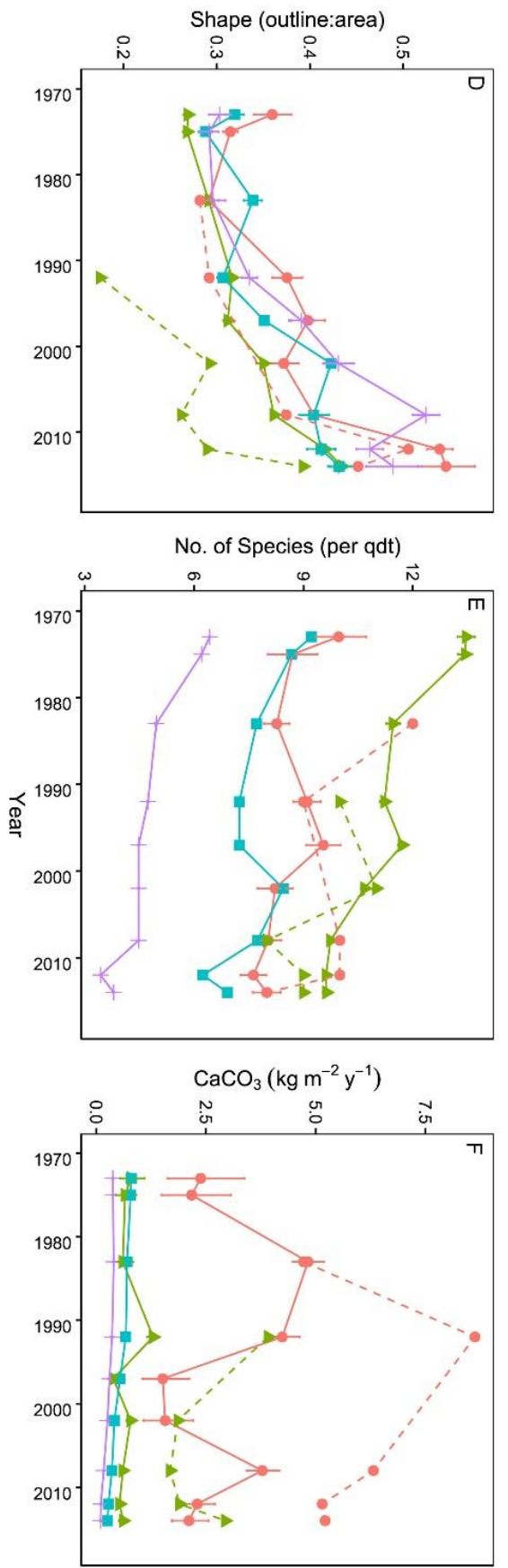
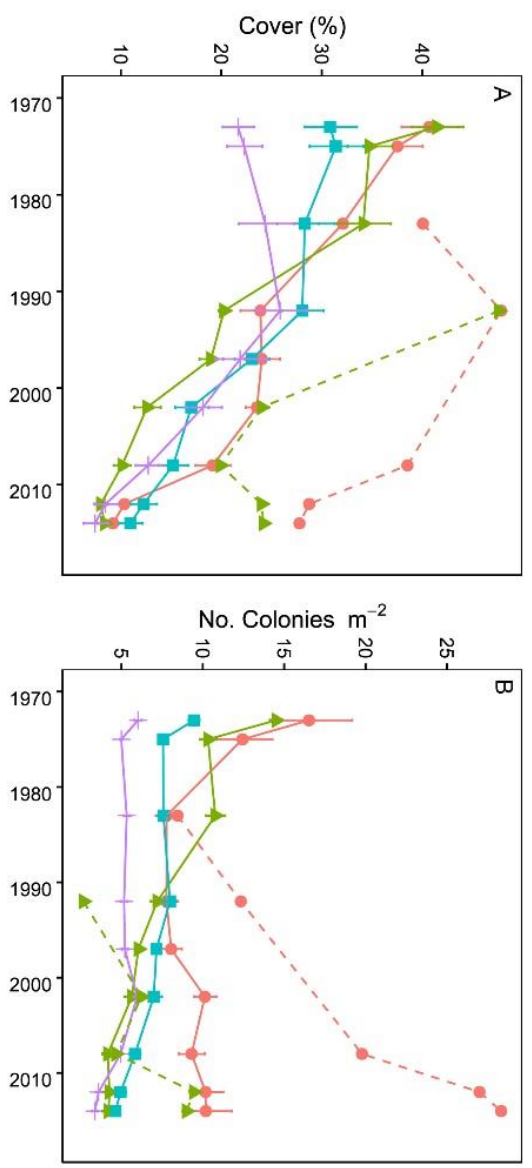

Mean colony size $\left(\mathrm{cm}^{2}\right)$

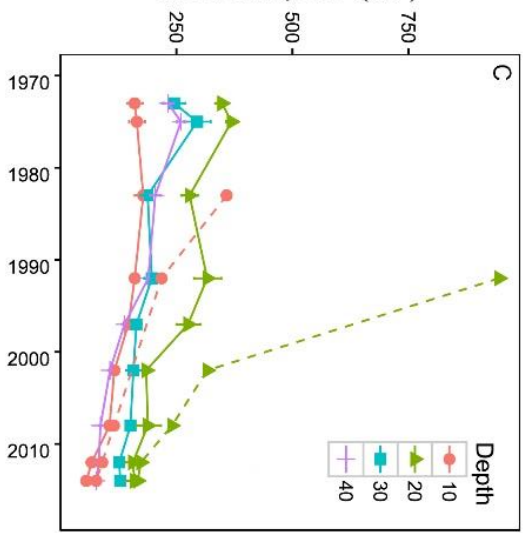

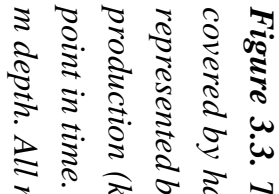

इ

ई $\frac{5}{5} \frac{5}{5} \frac{5}{5}$

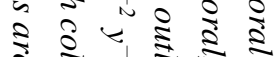

ร)

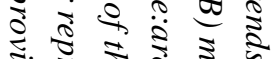

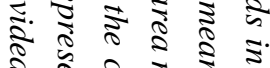

$\sqrt[3]{3} \lesssim$

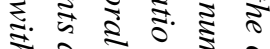

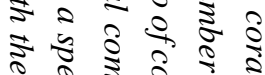

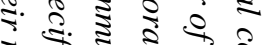

के ते ई

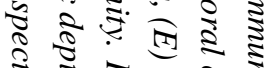

‡.

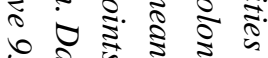

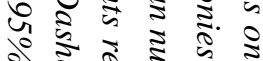

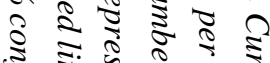

จ.

ร) $\rightarrow$ ร

ร)

₹.

ริ)

ई

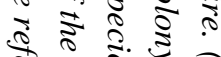

क.

ริ ฐ

施

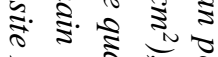

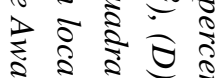

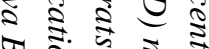

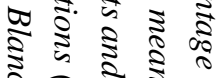

ร T $\stackrel{2}{5}$ क

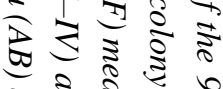

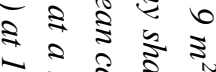

จ

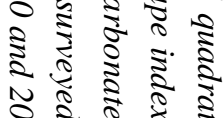




\begin{tabular}{|c|c|c|c|c|c|c|c|c|c|c|c|}
\hline \multirow[t]{2}{*}{ Site } & \multirow[t]{2}{*}{$\begin{array}{c}\text { Depth } \\
(\mathbf{m})\end{array}$} & \multicolumn{2}{|c|}{$\begin{array}{c}\text { Cover } \\
(\%)\end{array}$} & \multicolumn{2}{|c|}{$\begin{array}{l}\text { Number of } \\
\text { colonies } \mathbf{m}^{-2}\end{array}$} & \multicolumn{2}{|c|}{$\begin{array}{c}\text { Number of } \\
\text { species }\end{array}$} & \multicolumn{2}{|c|}{ Skewness } & \multicolumn{2}{|c|}{ Kurtosis } \\
\hline & & 1973 & 2014 & 1973 & 2014 & 1973 & 2014 & 1973 & 2014 & 1973 & 2014 \\
\hline I & 10 & 48.5 & 1.1 & 54 & 4 & 5 & $3(1)$ & 6.4 & 1.5 & 55.9 & 4.8 \\
\hline II & & 22.7 & 5.9 & 8 & 6 & 10 & $10(4)$ & 2.4 & 3.3 & 8.4 & 13.9 \\
\hline III & & 37.0 & 24.0 & 11 & 27 & 13 & $13(1)$ & 3.7 & 4.2 & 19.5 & 26.5 \\
\hline IV & & 62.8 & 22.2 & 12 & 14 & 14 & $9(1)$ & 3.4 & 3.2 & 16.0 & 15.4 \\
\hline $\begin{array}{c}\text { AB } \\
(\mathbf{1 9 8 3})\end{array}$ & & 40.1 & 27.8 & 76 & 225 & 12 & 8 & 4.6 & 4.9 & 24.5 & 36.0 \\
\hline I & 20 & 34.6 & 8.7 & 18 & 4 & 13 & 11 & 4.1 & 5.5 & 23.6 & 32.0 \\
\hline II & & 32.9 & 5.6 & 14 & 2 & 15 & 7 & 7.5 & 1.3 & 67.4 & 3.1 \\
\hline III & & 34.9 & 16.6 & 12 & 7 & 12 & $11(1)$ & 2.2 & 3.9 & 8.2 & 21.8 \\
\hline IV & & 70.6 & 5.6 & 14 & 6 & 14 & 10 & 2.1 & 3.7 & 8.1 & 18.2 \\
\hline $\begin{array}{c}\mathbf{A B} \\
(1992)\end{array}$ & & 47.6 & 24.2 & 24 & 81 & 10 & 9 & 4.4 & 4.2 & 20.7 & 22.9 \\
\hline I & 30 & 22.4 & 4.4 & 7 & 3 & 10 & $8(1)$ & 3.1 & 4.3 & 13.6 & 20.9 \\
\hline II & & 19.7 & 14.2 & 8 & 5 & 8 & $8(2)$ & 3.4 & 3.5 & 16.5 & 17.2 \\
\hline III & & 31.0 & 9.6 & 12 & 5 & 9 & 7 & 4.8 & 2.4 & 29.6 & 8.8 \\
\hline IV & & 60.0 & 20.2 & 11 & 5 & 10 & 5 & 2.5 & 2.5 & 8.6 & 9.9 \\
\hline I & 40 & 12.9 & 1.4 & 6 & 2 & 7 & 4 & 4.3 & 2.1 & 22.5 & 7.4 \\
\hline II & & 17.6 & 6.9 & 5 & 4 & 6 & 6 & 4.1 & 3.1 & 21.1 & 11.2 \\
\hline III & & 36.1 & 18.4 & 8 & 4 & 8 & 2 & 3.3 & 3.1 & 14.0 & 14.6 \\
\hline IV & & 25.0 & 11.3 & 5 & 3 & 5 & $4(1)$ & 5.3 & 2.6 & 32.7 & 10.2 \\
\hline
\end{tabular}

$\begin{array}{ccccc}\text { Site } & \begin{array}{c}\text { Depth } \\ (\mathbf{m})\end{array} & \begin{array}{c}\text { Mean colony size } \\ \left(\mathrm{cm}^{2}\right)\end{array} & \begin{array}{c}\text { Shannon } \\ \text { diversity }\end{array} & \begin{array}{l}\text { Simpson } \\ \text { diversity }\end{array}\end{array}$

Evenness diversity
$\mathrm{CaCO}_{3}$ production $(\mathrm{kg}$ $\left.\mathbf{m}^{-2} \mathbf{y}^{-1}\right)$

\begin{tabular}{c|c|c|c|c|c|c|c|c|c|c|c}
\hline & & $\mathbf{1 9 7 3}$ & $\mathbf{2 0 1 4}$ & $\mathbf{1 9 7 3}$ & $\mathbf{2 0 1 4}$ & $\mathbf{1 9 7 3}$ & $\mathbf{2 0 1 4}$ & $\mathbf{1 9 7 3}$ & $\mathbf{2 0 1 4}$ & $\mathbf{1 9 7 3}$ & $\mathbf{2 0 1 4}$ \\
\hline I & 10 & 47.5 & $\mathbf{2 4 . 1}$ & 0.45 & 0.62 & 0.25 & 0.38 & 0.13 & 0.18 & 12.95 & 0.28 \\
\hline II & & 143.0 & $\mathbf{3 1 . 8}$ & 1.69 & 1.81 & 0.75 & 0.78 & 0.48 & 0.52 & 2.37 & 0.51 \\
\hline III & & 141.6 & $\mathbf{3 1 . 7}$ & 1.77 & 1.47 & 0.75 & 0.65 & 0.51 & 0.42 & 3.86 & 4.86 \\
\hline IV & & 206.0 & $\mathbf{1 0 7 . 5}$ & 1.31 & 1.33 & 0.54 & 0.65 & 0.37 & 0.38 & 7.78 & 4.51 \\
\hline AB & & & & & & & & & & \\
$(\mathbf{1 9 8 3})$ & & 273.6 & $\mathbf{4 5 . 4}$ & 1.32 & 1.15 & 0.58 & 0.62 & 0.37 & 0.33 & 4.7 & 5.2 \\
\hline I & 20 & 118.5 & 61.8 & 2.13 & 1.45 & 0.85 & 0.62 & 0.61 & 0.41 & 2.07 & 0.42 \\
\hline II & & 106.0 & 140.8 & 2.31 & 1.57 & 0.87 & 0.76 & 0.66 & 0.45 & 1.66 & 0.22 \\
\hline III & & 150.4 & 130.1 & 2.03 & 1.59 & 0.84 & 0.72 & 0.58 & 0.46 & 1.45 & 0.68 \\
\hline IV & & 339.0 & $\mathbf{5 4 . 1}$ & 1.97 & 2.11 & 0.80 & 0.86 & 0.56 & 0.60 & 2.70 & 0.26 \\
\hline AB & & & & & & & & & & \\
$(\mathbf{1 9 9 2})$ & & 516.7 & $\mathbf{7 0 . 4}$ & 1.20 & 1.50 & 0.53 & 0.71 & 0.34 & 0.43 & 3.72 & 2.94 \\
\hline I & 30 & 152.6 & $\mathbf{4 7 . 0}$ & 1.88 & 1.54 & 0.80 & 0.70 & 0.54 & 0.44 & 0.69 & 0.13 \\
\hline II & & 113.0 & 108.0 & 1.44 & 1.27 & 0.68 & 0.60 & 0.41 & 0.36 & 0.54 & 0.34 \\
\hline III & & 91.3 & 94.5 & 0.99 & 1.07 & 0.42 & 0.52 & 0.28 & 0.31 & 0.65 & 0.20 \\
\hline IV & & 259.5 & 243.2 & 1.28 & 0.59 & 0.59 & 0.30 & 0.37 & 0.17 & 1.52 & 0.44 \\
\hline I & 40 & 110.4 & 39.2 & 1.32 & 0.98 & 0.64 & 0.58 & 0.38 & 0.28 & 0.30 & 0.03 \\
\hline II & & 91.0 & $\mathbf{2 6 . 5}$ & 0.86 & 0.70 & 0.40 & 0.34 & 0.25 & 0.20 & 0.30 & 0.09 \\
\hline III & & 121.0 & 138.9 & 0.46 & 0.16 & 0.18 & 0.07 & 0.13 & 0.05 & 0.46 & 0.20 \\
\hline IV & & 206.5 & 117.0 & 1.21 & 0.58 & 0.63 & 0.35 & 0.34 & 0.17 & 0.48 & 0.14 \\
\hline
\end{tabular}


Table 3.3. Summary of coral community characteristics. Percentage coral cover of each $9 \mathrm{~m}^{2}$ quadrat; number of colonies per square meter; number of species present per quadrat, newly appeared species in 2014 are given in parenthesis; skewness; kurtosis and mean colony size $\left(\mathrm{cm}^{2}\right)$ over all colonies that were present in the quadrat. A significant (Post Hoc test, $p<0.05$ ) decline in the mean colony size in an individual quadrat over time is indicated by a bold value in 2014. Community diversity indices include: Shannon-Wiener diversity index, Simpson index and Pielou's evenness scores for species contribution. Estimated coral calcium carbonate $\left(\mathrm{CaCO}_{3}\right)$ production in $\mathrm{kg} \mathrm{m}^{-2} y^{-1}$ of the coral community. All characteristics are given for 1973 and 2014 by depth and site (I-IV). For the reference site AB values are provided for their respective first year of visit: $10 \mathrm{~m}$ (1983), $20 \mathrm{~m}$ (1992).

In 1973 the coral community on the shallow fore-reef $(10 \mathrm{~m}$ and $20 \mathrm{~m})$ at sites I-IV was overall dominated by Orbicella spp. (28\%), A. agaricites (20\%) and M. mirabilis (17\%) (Appendix 3.B). By 2014, M. mirabilis had become the dominant species (28\%), followed by Orbicella complex (16\%) and A. lamarcki (12\%). The latter confirms the absence of obvious shifts in species composition but instead a general decline in cover of all species. It does, however, also show the increased relative importance of $M$. mirabilis with respect to other species at the most shallow quadrats over the more recent years (Figure 3.4). At $10 \mathrm{~m}$ depth evenness increased in most quadrats in 2014 compared to 1973 (Table 3.3) which is indicative of a more equal contribution of the species present to total coral cover. This is largely a consequence of the decline in cover of the both initially dominant M. mirabilis and Orbicella complex (Figure 3.3). On the deep fore-reef ( 30 and $40 \mathrm{~m}$ ) the coral community was dominated by A. lamarcki throughout the studied period (63\% in 1973, 77\% in 2014) resulting in generally low evenness scores (Table 3.3). In 1973, Montastraea cavernosa (13\%), Siderastrea siderea (6\%), and Stephanocoenia michelini (6\%) followed A. lamarcki in cover. These same species were still dominating the community in 2014: M. cavernosa (8\%), S. michelini (6\%) and S. siderea (3\%), but all had declined in total cover.

Some species appear relatively persistent, and include both framework building species (Colpophyllia natans, S. michelini, Meandrina meandrites, Diploria strigosa) and opportunistic species (M. mirabilis, A. lamarcki, Madracis complex, Porites astreoides). These species showed either an increase in cover, a decrease of less than $10 \%$ or they had newly emerged in multiple quadrats. An increase in the cover of certain species over time was almost exclusively observed on the shallow reef and no species consistently increased in all quadrats in which it was initially present (Appendix 3.B). Species that were dominant in 1973 but declined consistently in the majority of the quadrats included: Orbicella complex, Eusmilia fastigiata, M. cavernosa, Mycetophyllia spp., S. siderea, A. agaricites, M. complex, M. meandrites, S. michelini (Appendix 3.B). Comparable to the main quadrats (I-IV), the Orbicella spp. and $M$. cavernosa declined strongly at both depths at AB. At $10 \mathrm{~m}$ there was 


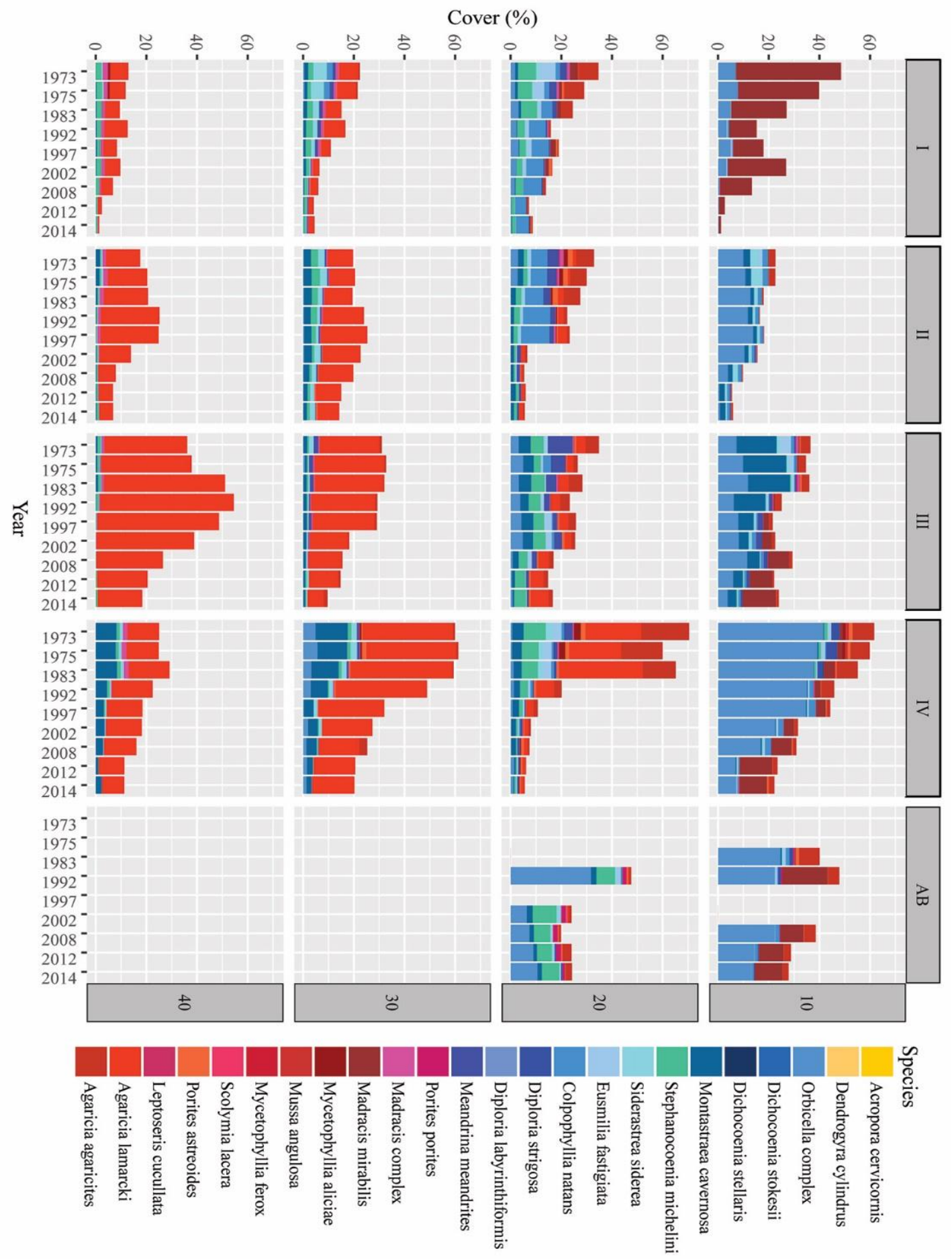


Figure 3.4. Spatio-temporal dynamics in coral community composition on Curaçao and Bonaire. Bars represent the coral cover at surveyed points in time in the $9 \mathrm{~m}^{2}$ quadrats. Bars are subdivided (stacked) by species. Competitive species are given in shades of yellow (virtually absent), framework building species in blue and opportunistic species in red/purple. Sites are listed as I-IV (main sites) and AB (reference site Awa Blancu) indicated along the top and the depths (10,20,30, and $40 \mathrm{~m})$ given on the right side of the panel. Detailed characteristics per quadrat can be found in Appendices 3.B and 3.C.

a clear increase in cover of $M$. mirabilis and a less distinct increase in M. meandrites. All other species showed a considerable decline. At $20 \mathrm{~m}$, with the exception of Porites porites, S. siderea and $S$. michelini, all species declined in cover. A particularly strong decline occurred in the Orbicella complex (71\%) and C. natans (100\%). Nevertheless, Orbicella spp. still covered 12\% of the total quadrat at both depths in 2014.

\section{Temporal Change in Coral Calcium Carbonate Production}

The consistent decline in coral cover inherently caused a reduction in the estimated rates of coral carbonate production in 2014 compared to 1973 (Figure 3.3F). The estimated mean contribution of corals to carbonate production $\left(\mathrm{kg} \mathrm{CaCO} 3 \mathrm{~m}^{-2} \mathrm{y}^{-1}\right)$ over all quadrats declined by $72 \%$ from a mean of $2.5 \mathrm{~kg} \mathrm{~m}^{-2} \mathrm{y}^{-1}$ in 1973 to $0.8 \mathrm{~kg} \mathrm{~m}^{-2} \mathrm{y}^{-1}$ in in 2014 , respective $95 \%$ confidence intervals: $0.7-4.3$ and $0.02-1.6$. The three species that accounted for the highest cover also contributed most prominently to the estimated coral calcification: M. mirabilis, Orbicella complex and A. lamarcki in both 1973 and 2014. In 1973, coral carbonate production was highest at $10 \mathrm{~m}$ depth (Figure 3.3F \& Table 3.3), with a mean of $6.7 \mathrm{~kg} \mathrm{~m}^{-2} \mathrm{y}^{-1}$, largely due to the high cover of the two rapidly calcifying species $M$. mirabilis (rate: $29 \mathrm{~kg} \mathrm{~m}^{-2} \mathrm{y}^{-1}$ ) and Orbicella spp. (rate: $14 \mathrm{~kg} \mathrm{~m}^{-2} \mathrm{y}^{-1}$ ) (Table 3.3). Particularly the $97 \%$ decline in Orbicella complex cover has resulted in a considerable decrease in calcification by 2014. Total coral calcification decreased with depth (Figure 3.3F) and at different depths a decline occurred over time (Table 3.3). The reduction in coral cover at $20 \mathrm{~m}$ depth at $\mathrm{AB}$ resulted in a decline in estimated coral calcification from $3.7 \mathrm{~kg} \mathrm{~m}^{-2} \mathrm{y}^{-1}$ (1983) to $2.9 \mathrm{~kg} \mathrm{~m}^{-2} \mathrm{y}^{-1}$ (2014), which is still high compared to sites I-IV. Due to the vast increase in M. mirabilis at AB the total estimated coral calcification at $10 \mathrm{~m}$ had increased here slightly since 1983 (Table 3.3).

Figure 3.5. Colony size frequency distribution for the three most prominent species: Orbicella spp., Agaricia lamarcki and Madracis mirabilis. Total number of colonies in $9 \mathrm{~m}^{2}$ quadrats in 1973 (start time series) and 2014 (end) summed over the four main sites (I-IV) on a log scale (8 size classes), at different depths (when the species was present). Scale size varies for the different species. Species colour codes correspond to the colours given in Figure 3.4. For supporting data, also on the other species, see Appendices 3.B, 3.C and 3.D. 


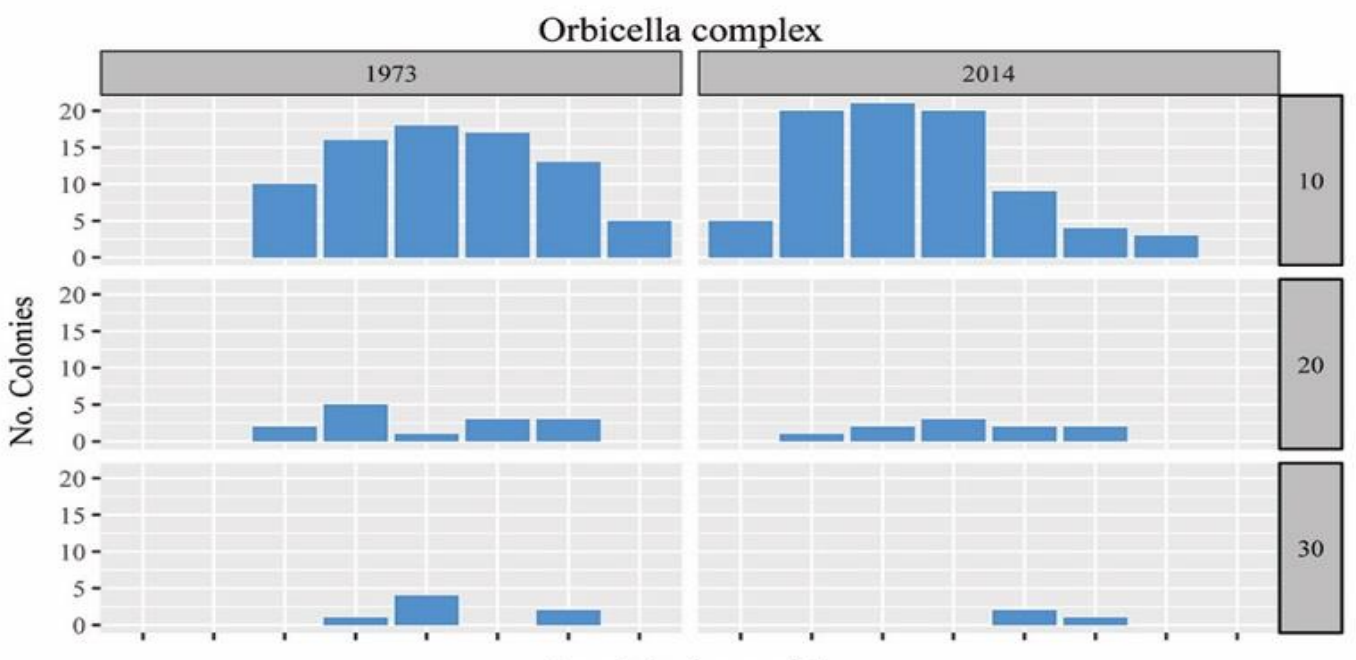

Agaricia lamarcki

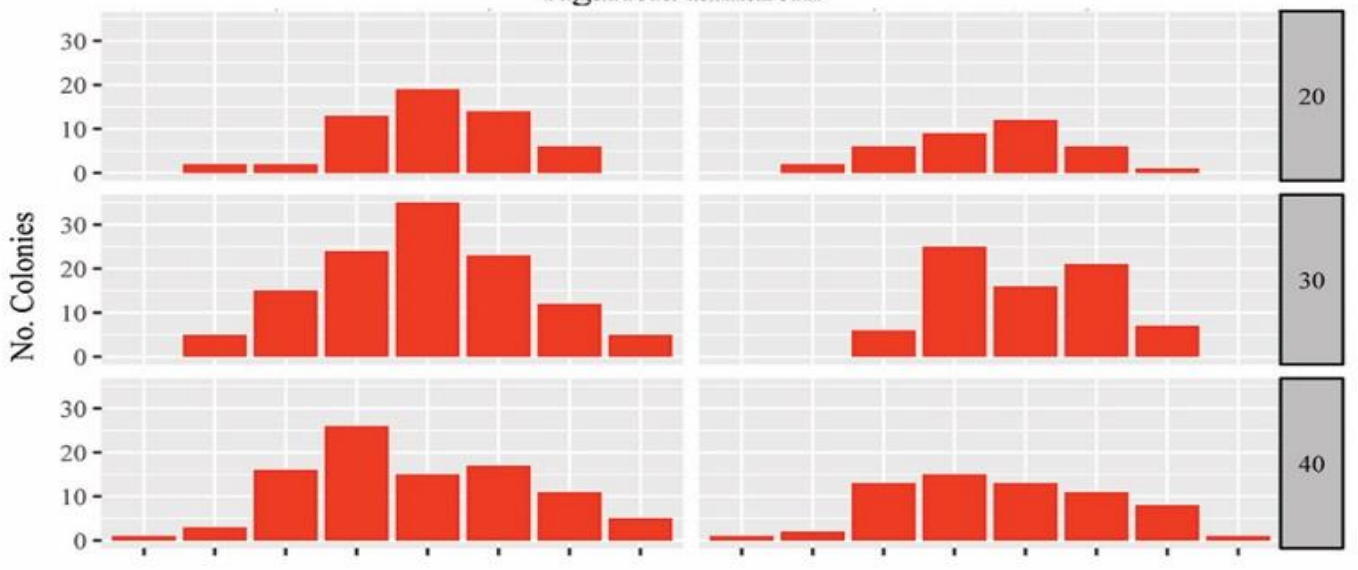

Madracis mirabilis

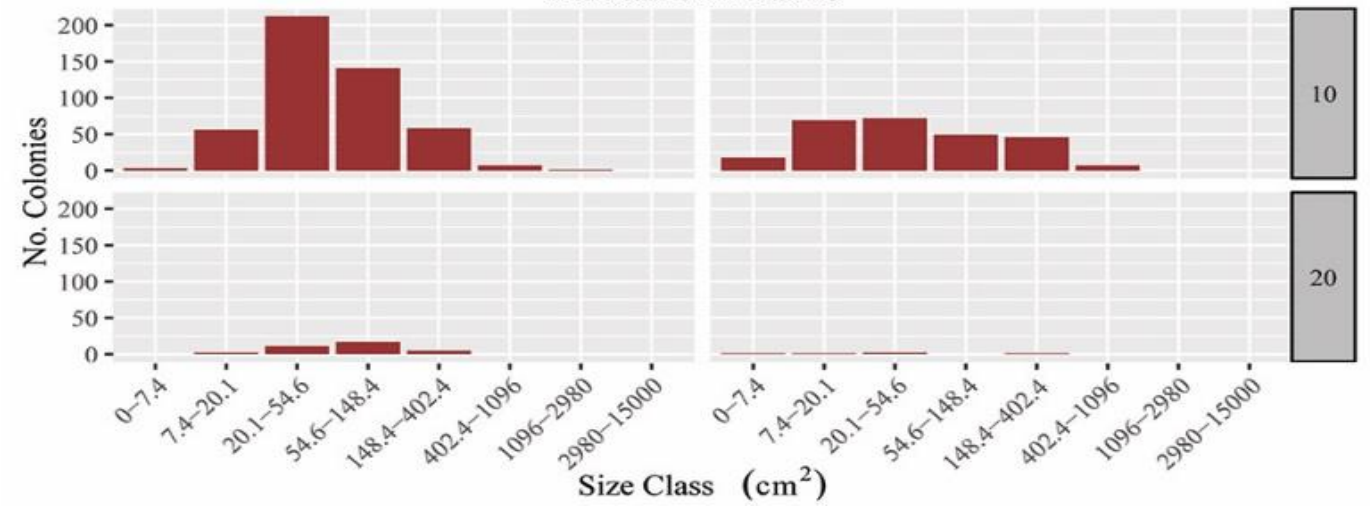


Table 3.4. Species specific life-history strategy and cover. Overview of all encountered species with their respective life history strategy and mean contribution (percentage) to the total coral cover at the start (1973) and end (2014) across the four main time series (I-IV). A cover of zero percent at 1973 and 2014 means the species was observed at some point between both years. Detailed characteristics per quadrat can be found in Appendices 3.B and 3.C.

\begin{tabular}{l|lc|c|}
\multirow{2}{*}{ Species } & \multirow{2}{*}{ Life-history strategy } & \multicolumn{2}{c}{ Cover (\%) } \\
\cline { 3 - 4 } & & $\mathbf{1 9 7 3}$ & $\mathbf{2 0 1 4}$ \\
\hline Acropora cervicornis & Competitive & 0 & 0 \\
\hline Dendrogyra cylindrus & Competitive & 0.01 & 0 \\
\hline Orbicella complex & Framework building & 14.1 & 9.8 \\
\hline Dichocoenia stokesii & Framework building & 0.06 & 0 \\
\hline Dichocoenia stellaris & Framework building & 0 & 0 \\
\hline Montastraea cavernosa & Framework building & 10.6 & 7.6 \\
\hline Stephanocoenia michelini & Framework building & 6.4 & 9.1 \\
\hline Siderastrea siderea & Framework building & 4.3 & 3.1 \\
\hline Eusmilia fastigiata & Framework building & 3.3 & 0.7 \\
\hline Colpophyllia natans & Framework building & 2.7 & 5.1 \\
\hline Diploria strigosa & Framework building & 0.81 & 0.35 \\
\hline Diploria labyrinthiformis & Framework building & 0.02 & 0 \\
\hline Meandrina meandrites & Framework building & 4.2 & 2.3 \\
\hline Porites porites & Opportunistic & 0.08 & 0 \\
\hline Madracis complex & Opportunistic & 2.4 & 0.8 \\
\hline Madracis mirabilis & Opportunistic & 8.1 & 17.6 \\
\hline Mycetophyllia aliciae & Opportunistic & 1.2 & 0.25 \\
\hline Mussa angulosa & Opportunistic & 0.16 & 0 \\
\hline Mycetophyllia ferox & Opportunistic & 0.1 & 0 \\
\hline Scolymia lacera & Opportunistic & 0 & 0 \\
\hline Porites astreoides & Opportunistic & 1.4 & 2.3 \\
\hline Leptoseris cucullata & Opportunistic & 0 & 0 \\
\hline Agaricia lamarcki & Opportunistic & 29.8 & 35.8 \\
\hline Agaricia agaricites & Opportunistic & 9.9 & 5.2 \\
\hline & & & 0 \\
\hline
\end{tabular}

\section{Discussion}

Since the early 1970s the coral communities of Curaçao and Bonaire have changed significantly in coral cover and species composition. Total cover has declined and colonies have generally become smaller on both the shallow $(10-20 \mathrm{~m})$ and upper-mesophotic reef $(30-40 \mathrm{~m})$. In particular, initially dominant framework building species such as Orbicella spp., M. cavernosa and $S$. siderea, lost significant cover, a trend that occurs throughout the wider Caribbean region 
(Bruckner and Bruckner 2006; Edmunds and Elahi 2007; Jackson et al. 2014). Concurrently, the relative cover of some phenotypically plastic so-called weedy species (sensu Knowlton 2001) moderately increased since 1973 . The observed shift also took place at the reference site Awa Blancu (AB) which is located far upstream and relatively distant from anthropogenic disturbances along the coast of Curaçao (Figure 3.1), but less distinct.

Overall, reef degradation was less pronounced at Awa Blancu (Figure 3.2). A noticeable difference with the shallow reef of sites I-IV was that Orbicella, over time, remained the dominant genus at $\mathrm{AB}$ with still relatively high cover by 2014 (13\% and $11 \%$ of the total quadrat at 10 and $20 \mathrm{~m}$ respectively). Moreover, the coral communities at $\mathrm{AB}$ remained dominated by framework building species throughout the times series. This is in contrast to sites I-IV, where opportunistic species have become dominant. The latter suggests that coral communities along the leeward coast near urban areas and outlet bays (I-IV) receive additional stress on top of stress factors acting on a larger scale (i.e., regional or worldwide), at least down to $20 \mathrm{~m}$ depth. Since the start of our time series both Curaçao and Bonaire have experienced significant human population expansion (50\% increase on Bonaire since 2001) as well as increased tourism (CBS Curaçao 2015, http://www.cbs.cw; CBS Dutch Caribbean 2015 www.rijksdienstcn.com). Both have consequently led to extensive development of the coastal area. Factors associated with coastal development such as pollution, turbidity and sedimentation negatively impact coral recruitment and survival by smothering corals or reducing the ability of corals to cope with diseases, bleaching or spatial competition (e.g. Meesters et al. 1992; Nugues and Roberts 2003b; Fabricius 2005; Jackson et al. 2014; Vega-Thurber et al. 2014). Similar to many tropical regions, sewage water on Curaçao and Bonaire is generally insufficiently treated or even largely untreated before it is discharged (Buth and Ras 1992; Lapointe and Mallin 2011). Indeed, along the coast of Curaçao clearly higher nutrient concentrations have been found at sites I-III compared to Fuik bay, which is located near Awa Blancu (Gast et al. 1999; Govers et al. 2014). Elevated nutrient levels have also been found at Karpata (IV) on Bonaire during measurements in 2012 and 2013 (Slijkerman et al. 2014). The observed spatial variation in coral community degradation may therefore reflect the impact of persevering stress factors associated with coastal urbanization around more densely populated areas. These results suggest that implementation of thorough local conservation and management strategies, such as the implementation of properly working sewage treatment plants, will benefit local reef health and retard reef degradation. In addition, stronger recreational diving regulation may be useful since approximately $75 \%$ of the stay-over tourists on Bonaire are divers (IVM report 2013; http://www.rijksdienstcn.com), undoubtedly having a local impact on the reef (e.g., Hawkins et al. 1999; Lamb et al. 2014). Comparable data are lacking for Curaçao. It is clear that supporting data on specific effects of the various local stressors will be essential for proper implementation of conservation strategies, but at present such data is very limited or even absent.

The observed general decline in cover and abundance of corals not only occurred among reef building species, but included also most opportunistic species (e.g., P. porites, Mycetophyllia spp., A. agaricites and Madracis spp.). A few species, in particular M. mirabilis, A. lamarcki and $P$. astreoides, however, appear to be more resilient and their decrease in cover and 
abundance was less distinct (Figure 3.4 \& Appendix 3.B). This is confirmed by observations throughout the Caribbean (Green et al. 2008; Anderson et al. 2014; Perry et al. 2015a). Nevertheless, these species seem to decline when the overall coral cover in the quadrat was substantially reduced. Like many coral species, M. mirabilis cannot easily settle on loose substratum such as sand or rubble (Bak and Criens 1981; Nagelkerken et al. 2000). Presumably, M. mirabilis relies on substratum provided by other species after mortality to settle or expand. Indeed, we found an increase in cover of $M$. mirabilis in quadrats where large colony building species (e.g., Orbicella complex) used to be dominant but died and left available hard substratum. Contrastingly, in quadrats where larger species were not initially present the cover of M. mirabilis remained low or even declined (e.g., site I, $10 \mathrm{~m}$ ). This implies that survival of this species is all but certain. According to predictions (e.g., Darling et al. 2012), several larger framework building species such as $S$. michelini and M. meandrites indeed appear to be relatively persistent (stress-tolerant sensu Darling et al. 2012) and were not readily lost over time. Though their average cover declined in all quadrats, they continued to contribute to total quadrat cover by 2014 (Figure 3.3). P. strigosa even newly appeared in several shallow quadrats and seems relatively stress-tolerant (Figure 3.3 \& Appendix 3.B). If, however, the observed declining trend continues, it is likely that even these species will disappear. Also, it remains to be seen whether the apparently opportunistic weedy species will be sufficiently tolerant to cope with the generally expected increase in frequency and severity of catastrophic events (Van Woesik et al. 2011; McClanahan et al. 2014). Historic records (e.g., van Woesik et al. 2012) show that even opportunistic species can become regionally extinct, as a consequence of largescale climate disturbances. At the present rate of decline (Figure 3.3A) total coral cover on the reef slopes of Curaçao and Bonaire may have dropped below $1 \%$ by 2030 .

Evidently, the decline in colony size and shift toward dominance of smaller colonies is largely caused by a decline in abundance of species that potentially form large colonies such as the Orbicella spp. (Figure 3.5). This was most apparent on AB at $20 \mathrm{~m}$ which was originally dominated by many large Orbicella colonies (Appendix 3.C). The observed shift in colony size of Orbicella, A. lamarcki and most other species is largely a result of partial mortality of large colonies. Coral growth or tissue regeneration may be impeded by increased spatial competition between corals and other benthic organisms such as algae or benthic cyanobacterial mats (Hughes 1994). Meesters et al. (1997a) describe two types of lesion in coral tissue: the so-called type I, where the lesion is completely enclosed by tissue; and type II, most frequently observed, where the lesion is located at the edge of the colony. The observed increase in the ratio of colony outline and live surface area of coral colonies (Table 3.3) could be an indication of the incapability of the coral to reclaim substratum after such a type II lesion (Meesters et al. 1997b). Partial mortality will generally result in a larger loss of surface area than colony outline, thus leading to a higher shape-ratio. A possible effect on colony size distribution caused by increased recruitment can be neglected since no new recruits were present in 2014. M. mirabilis may at present be the sole species to show recruitment within the quadrats. Fragmentation, however, makes it difficult to distinguish actual new M. mirabilis recruits. Nevertheless, the relative increase in abundance of opportunistic species in the past decade has contributed to a higher density of small colonies (Knowlton 2001; Darling et al. 2012). 
An important consequence of reduced coral cover is the loss of reef structural complexity and associated loss of biodiversity, but also coastal protection and human food security (e.g., Alvarez-Filip et al. 2009; Newman et al. 2015). In many of the studied quadrats, coral cover has approached or dropped below the $10 \%$ level. This point has been suggested as the threshold after which carbonate accretion will be surpassed by erosion and architectural complexity is being lost (Perry et al. 2013). Following this rationale, nine out of the 16 main quadrats would currently be in an eroding state (Table 3.3). Indeed, the estimated carbonate production has decreased considerably since 1973 in virtually all quadrats, largely attributed to the general reduction of coral cover and in particular the loss of large calcifying species such as Orbicella spp. (Perry et al. 2015a). The relative decline in carbonate production was highly comparable across depths, with the exception of $10 \mathrm{~m}$. At $10 \mathrm{~m}$ depth the degree by which carbonate production changed over time was generally lower and largely driven by the temporal dynamics in cover of the rapidly calcifying $M$. mirabilis. High $M$. mirabilis cover may compensate some of the reduction in production but it cannot be considered an important framework builder able to compensate for the loss of structural complexity provided by large calcifying species (such as Orbicella spp. and Acropora spp.) (Alvarez-Filip et al. 2011a). Moreover, opportunistic rapidly calcifying species such as $M$. mirabilis are typically absent at greater depths.

Diversity indices may reflect a gradient of decreased disturbance with greater depth. According to the intermediate disturbance theory highest community diversity is found at intermediate disturbance (Connell 1978). Over the depth range studied, highest diversity occurred at $20 \mathrm{~m}$ depth both in 1973 and 2014, which could be indicative of intermediate disturbance level. At 10 $\mathrm{m}$ depth, high disturbance likely impacted all but the most resistant species such as M. mirabilis or P. astreoides, resulting in a low diversity. Both natural (e.g., hurricanes) and anthropogenic disturbances (e.g., eutrophication and run-off) generally have the highest impact on the more shallow reef. Particularly the anthropogenic stress has intensified over the past decades likely putting more stress on the most shallow reef zones $(<20 \mathrm{~m})$. The generally low diversity of coral communities at greater depth $(>20 \mathrm{~m})$, in particular at $40 \mathrm{~m}$, might indicate that these reefs experience lower levels of disturbance (e.g., declined water quality, reduced herbivory and hurricanes). Following the intermediate disturbance theory, reefs with low disturbance are characterized by dominant competitors (Connell 1978). Indeed, the deeper reefs of Curaçao and Bonaire were dominated by A. lamarcki consistently between 1973 and 2014. In addition, low light intensities at greater depth likely support a generally lower diversity of species. Although, the reefs at greater depths may initially have experienced less disturbance (Bak et al. 2005) an obvious degrading trend was nevertheless observed, in particular since approximately 1990. A decline in cover and abundance of coral, including A. lamarcki may suggest that stress on these reefs has recently been increasing. Lapointe and Mallin (2011), for instance, found evidence for land-based sewage inputs reaching the deeper reef $(18 \mathrm{~m})$ near urban areas on Curaçao. Nutrient measurements on reefs below $18 \mathrm{~m}$ are virtually absent, but eutrophication may be reaching greater depths as well. The observed deep community dynamics of the recent years may, however, not be linked (anymore) to the absence of disturbance, but simply be the outcome of one strong competitor (A. lamarcki) and a generally limited pool of species at greater depths. 
The absence of weedy species such as M. mirabilis here may impede a shift to a more opportunistic coral community as seen on the shallow reef. Such species are simply unable to settle at these depths due to factors such as low light intensity. As yet no new opportunistic candidate has settled and with A. lamarcki, still dominant though steadily declining as well, the prospect for recovery of mesophotic coral communities on reefs appears to be very limited.

In conclusion, we found that coral communities of Curaçao and Bonaire have seen dramatic changes since 1973 . Overall coral communities were heavily degraded by 2014 . Phenotypically plastic and opportunistic species have increased and M. mirabilis, A. lamarcki, P. astreoides and $P$. strigosa may at present be considered very modest 'winners' among the Caribbean coral assembly. These species, however, are unlikely to remain 'winners' in a compromised habitat where suitable substratum for settlement is lost and disruptive events may become more frequent and severe, nor will they be able to provide the same structural complexity as the species from the near past (e.g. Orbicella spp.). The reefs at mesophotic depths $(30-40 \mathrm{~m})$ do not escape the general degradation of the coral community. Degradation was less evident away from urban areas. This suggests that local management, such as the provision of proper sewage treatment and run-off prevention, can have a positive effect on the general health and survival of coral reef communities. Although, the upsurge of some opportunistic species may provide a glimmer of hope for coral communities proper conservation and management will be essential to keep coral species from turning collectively into 'losers'.

\section{Acknowledgments}

We thank Alice Webb from The Royal Netherlands Institute for Sea Research (NIOZ) for her assistance with data organization and analyses. Furthermore, we are thankful for the continuous support received by the CARMABI research institute on Curaçao and STINAPA on Bonaire. The present research project was conducted by Wageningen University and Marine Research, formerly IMARES-WUR, at the request of and with funding from the Ministry of Economic Affairs for the purposes of Policy Support Research Theme "Analyse fotomateriaal koraalrif/fase 1" (project no. BO-11-019.02-038). The Royal Netherlands Institute for Sea Research (NIOZ) provided funding of exploitation costs and in kind support. Finally, we would like to extend our gratitude to both reviewers for their constructive review of the manuscript. 


\section{Appendix 3.A}

Second stage correlation matrix between sites at a specific depth. Rho-values indicate the degree of correlation. Significant Rho-values $(p<0.05)$ are given in bold and indicate a significantly similar pattern of community development among two quadrats at the same depth but different sites.

\begin{tabular}{|c|c|c|c|c|c|c|}
\hline Depth & Site & I & II & III & IV & $\mathrm{AB}$ \\
\hline \multirow[t]{5}{*}{$10 \mathrm{~m}$} & I & - & & & & \\
\hline & II & 0.842 & - & & & \\
\hline & III & 0.478 & 0.671 & - & & \\
\hline & IV & 0.647 & 0.850 & 0.882 & - & \\
\hline & $\mathrm{AB}$ & 0.142 & 0.029 & 0.371 & 0.371 & - \\
\hline \multirow[t]{5}{*}{$20 \mathrm{~m}$} & I & - & & & & \\
\hline & II & 0.771 & - & & & \\
\hline & III & 0.641 & 0.570 & - & & \\
\hline & IV & 0.791 & 0.867 & 0.572 & - & \\
\hline & $\mathrm{AB}$ & 0.429 & 0.314 & 0.714 & 0.657 & - \\
\hline \multirow[t]{4}{*}{$30 \mathrm{~m}$} & I & - & & & & \\
\hline & II & 0.869 & - & & & \\
\hline & III & 0.841 & 0.773 & - & & \\
\hline & IV & 0.796 & 0.761 & 0.625 & - & - \\
\hline \multirow[t]{4}{*}{$40 \mathrm{~m}$} & I & - & & & & \\
\hline & II & 0.625 & - & & & \\
\hline & III & 0.674 & 0.794 & - & & \\
\hline & IV & 0.733 & 0.628 & 0.830 & - & - \\
\hline
\end{tabular}

\section{Appendix 3.B}

Overview of initial (1973) and final (2014) cover of the individual species within a quadrat. Values provided as percentage cover of the total quadrat. Including the percentage change over time (negative values indicate a decline in cover). Species that were lost (Lost) or newly appeared (New). Species that appeared for a brief moment between 1973 and 2014 (-). The overview table can be accessed through the following link:

https://www.frontiersin.org/article/10.3389/fmars.2016.00247/full\#supplementary-material

\section{Appendix 3.C}

Overview of quadrat characteristics per species. Including: surface area (sum and mean), colony outline (sum and mean), number of colonies present, and shape (outline:area). The overview table can be accessed through the following link: 
Appendix 3.D

Coral colony size distribution. Sum of the number of colonies across the four main sites (excl. $\mathrm{AB})$ at five points in time. Colonies are distributed over 11 size classes on a log scale. Competitive species are given in shades of yellow, stress-tolerant species in blue and weedy species in red.

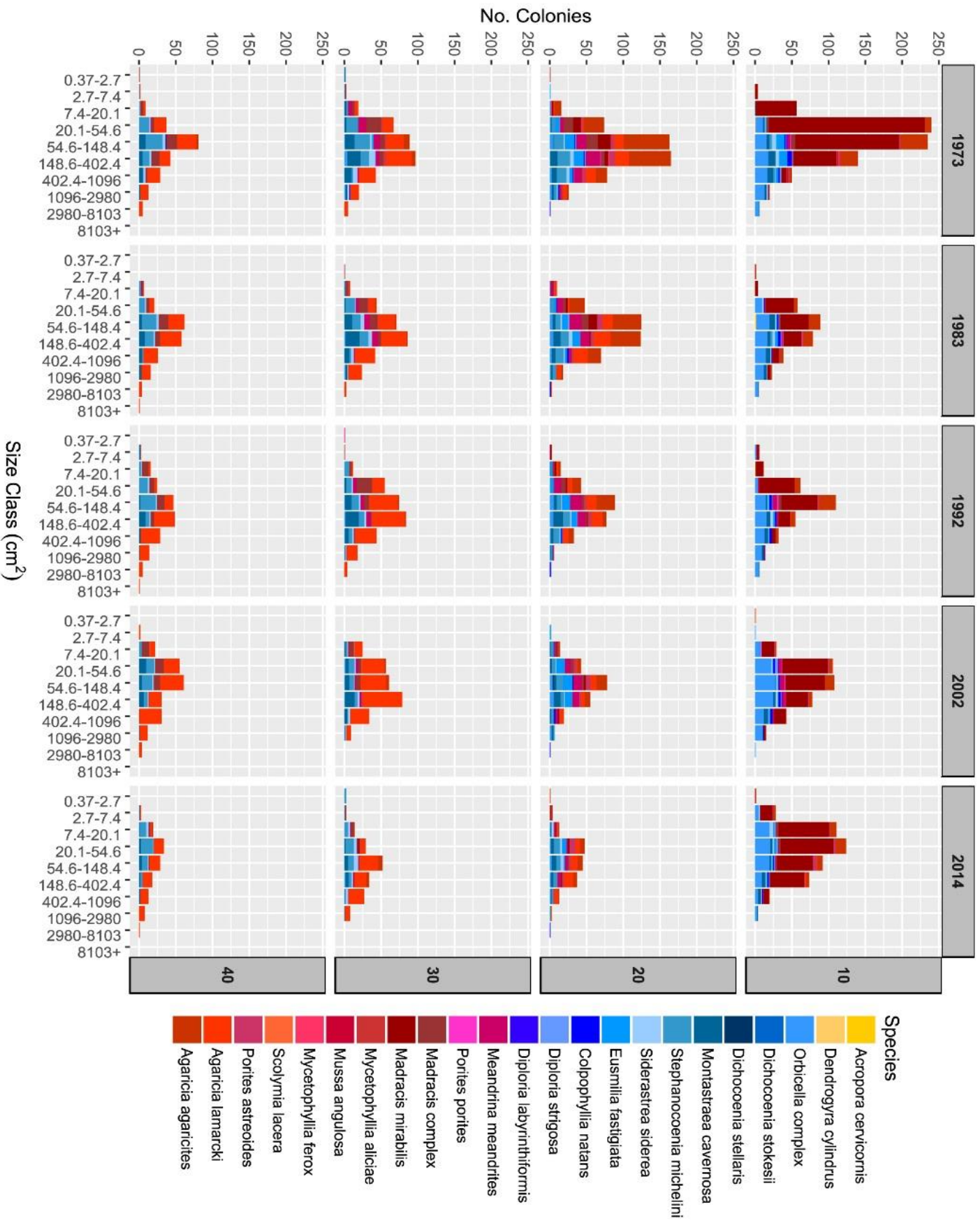




\section{1.

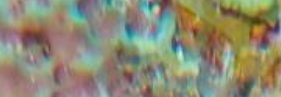

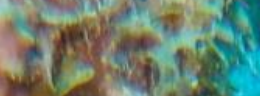

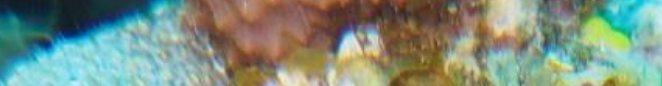

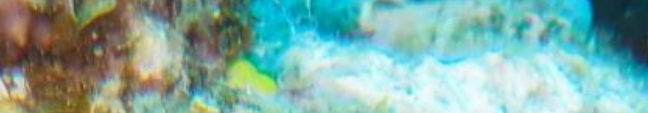
(6.

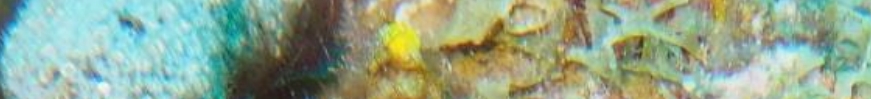

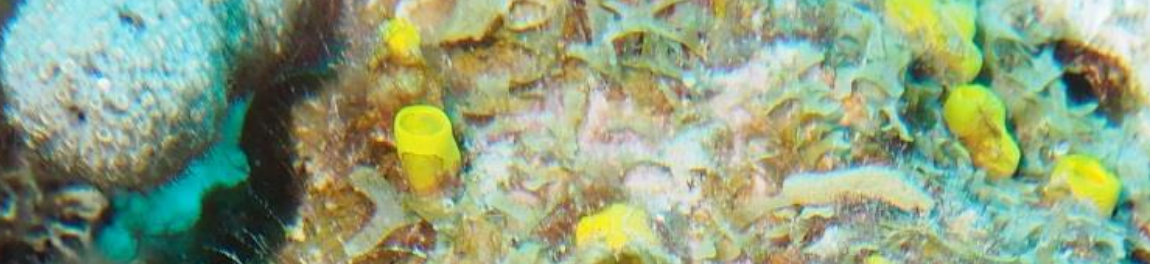
thas

siate the te

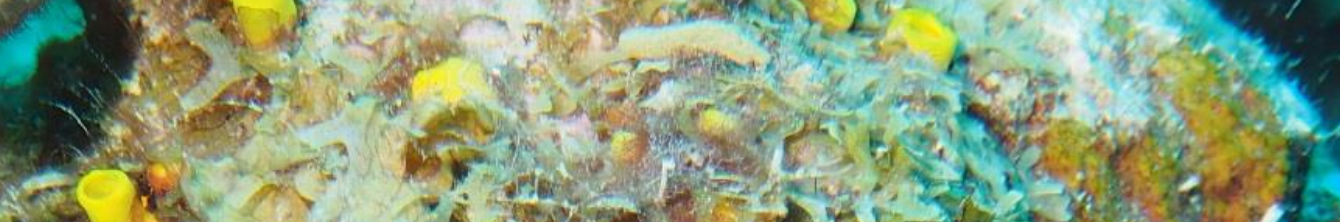

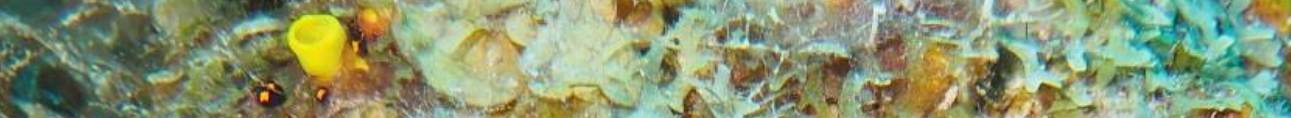

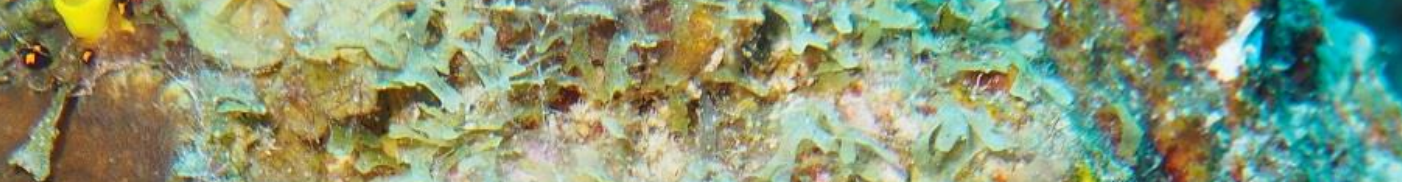
$\cos _{12}$ (1)

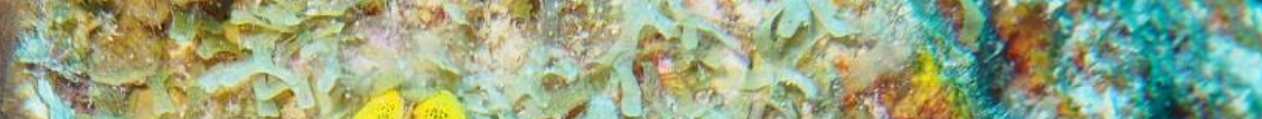

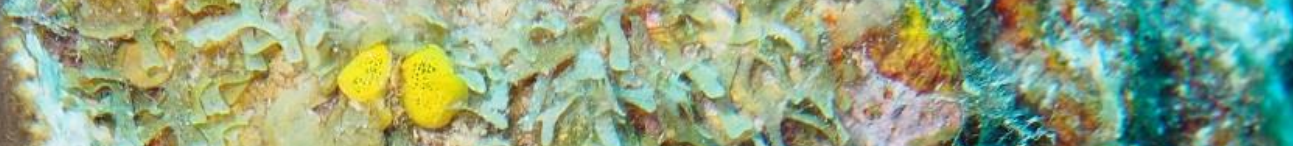
s.t.

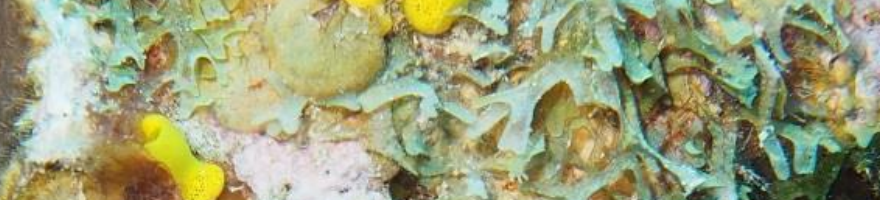

\section{$20+-\rightarrow$ at}

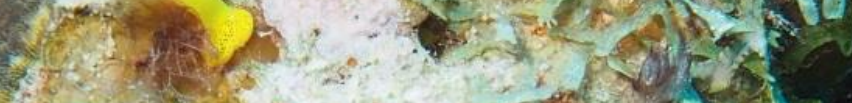

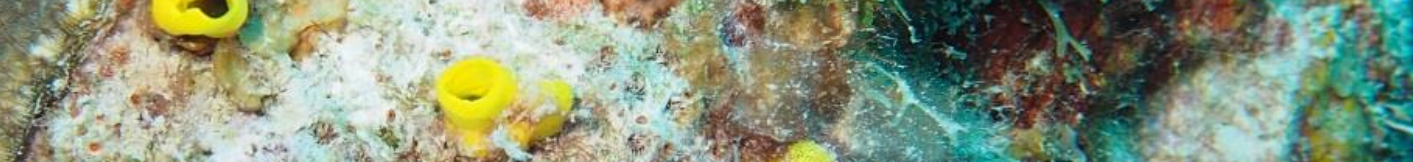

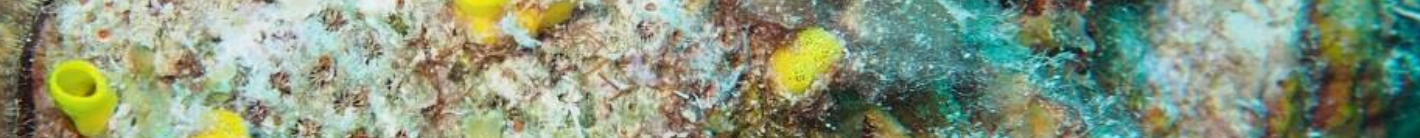

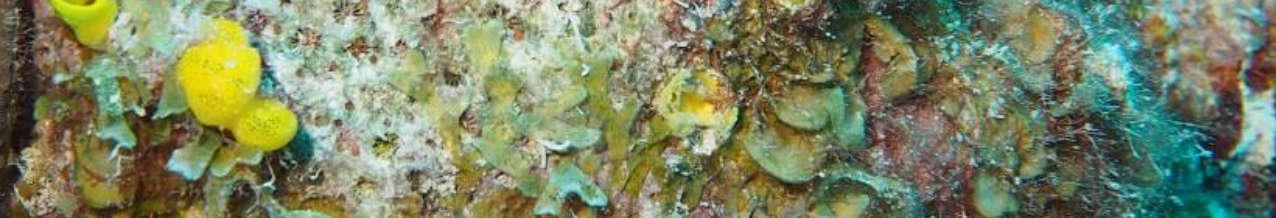

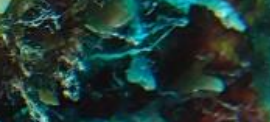

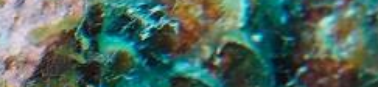

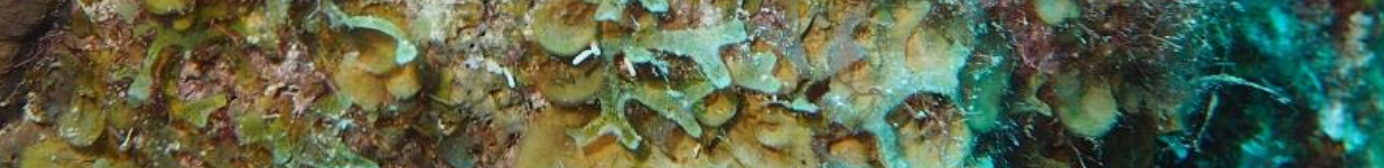
3.5.

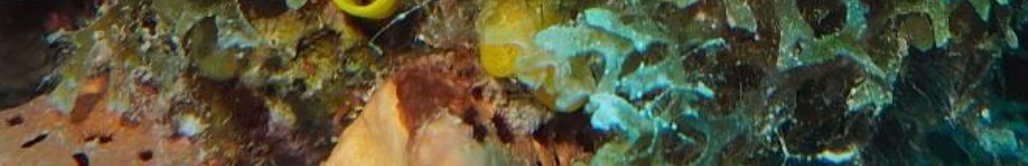
$\therefore+$ 


\title{
CHAPTER IV
}

\section{Quantification of chemical and mechanical bioerosion rates of six Caribbean excavating sponge species found on the coral reefs of Curaçao}

\author{
Didier M. de Bakker, Alice E. Webb, Lisanne A. van den Bogaart, Steven M. A. C. \\ van Heuven, Erik H. Meesters, Fleur C. van Duyl
}

PLoS ONE, 2018 : 13(5), 1-22 DOI: https://doi.org/10.1371/journal.pone.0197824

\begin{abstract}
Excavating sponges are among the most important macro-eroders of carbonate substrates in marine systems. Their capacity to remove substantial amounts of limestone makes these animals significant players that can unbalance the reef carbonate budget of tropical coral reefs. Nevertheless, excavating sponges are currently rarely incorporated in standardized surveys and experimental work is often restricted to a few species. Here were provide chemical and mechanical bioerosion rates for the six excavating sponge species most commonly found on the shallow reef of Curaçao (southern Caribbean): Cliona caribbaea, C. aprica, C. delitrix, C. amplicavata, Siphonodictyon brevitubulatum and Suberea flavolivescens. Chemical, mechanical and total bioerosion rates were estimated based on various experimental approaches applied to sponge infested limestone cores. Conventional standing incubation techniques were shown to strongly influence the chemical dissolution signal. Final rates, based on the change in alkalinity of the incubation water, declined significantly as a function of incubation time. This effect was mitigated by the use of a flow-through incubation system. Additionally, we found that mechanically removed carbonate fragments collected in the flow-through chamber $(1 \mathrm{~h})$ as well as a long-term collection method (1 week) generally yielded comparable estimates for the capacity of these sponges to mechanically remove substratum. Observed interspecific variation could evidently be linked to the adopted boring strategy (i.e. gallery-forming, cavity-forming or network-working) and presence or absence of symbiotic zooxanthellae. Notably, a clear diurnal pattern was found only in species that harbor a dense photosymbiotic community. In these species chemical erosion was substantially higher during the day. Overall, the sum of individually acquired chemical and mechanical erosion using flow-through incubations was comparable to rates obtained gravimetrically. Such consistency is a first in this field of research. These findings support the much needed confirmation that, depending on the scientific demand, the different approaches presented here can be implemented concurrently as standardized methods.
\end{abstract}




\section{Introduction}

The existence of tropical coral reefs relies on an ongoing biogenic precipitation of calcium carbonate $\left(\mathrm{CaCO}_{3}\right)$ by calcifying organisms (most importantly scleractinian corals) that is exceeding erosional forces (Goreau 1959a; Hutchings 1986; Glynn and Morales 1997; Kleypas et al. 2001; Erez et al. 2011; Andersson and Gledhill 2013; Glynn and Manzello 2015). Live corals, however, have been diminishing worldwide at unprecedented rates following phenomena such as reduced water quality, climate change and declined herbivorous pressure (e.g. Jackson et al. 2001; Hughes et al. 2003; Bellwood et al. 2004; Bak et al. 2005; Hoegh-Guldberg et al. 2007; Carpenter et al. 2008; De Bakker et al. 2017). Reefs in the Caribbean and Gulf of Mexico, in particular, have been heavily impacted in the past decades. Here coral cover was reduced, on average, to a mere 16\% (Gardner et al. 2003; Wilkinson 2008; Jackson et al. 2014). Alarmingly, the observed decline can largely be accredited to the mortality of formerly dominant frameworkbuilding species (i.e. Acropora spp. and Orbicella spp.) which are especially affected by direct or indirect anthropogenic stressors (Aronson and Precht 2001; Alvarez-Filip et al. 2011a; Perry et al. 2015a). Whilst the main reef-builders appear largely unable to cope with the current level of global and local disturbance (De Bakker et al. 2016b), the changing reef environment seems to favour, among others, bioeroding organisms (For an overview see Schönberg et al. 2017b: Table 3, Supplementary data S4).

Within the bioeroding community excavating sponges are frequently the dominant macroboring organisms (Hudson 1977; Rützler 2002; Sheppard et al. 2002). On Caribbean reefs sponges can be responsible for as much as $90 \%$ of the total macrobioerosion (Macgeachy and Stearn 1976). Murphy et al. (2016) describe two main strategies by which these sponges erode their substratum: (1) gallery-forming, where the sponge progressively works its way down, and (2) cavity-forming, where the sponge penetrates the limestone and forms chambers inside the substrate with often only the inhalant and exhalant fistulas being visible on the surface. Many gallery-forming sponges harbour high densities of dinoflagellate zooxanthellae (i.e. Symbiodinium spp.) and as such their boring strategy allows for optimal light exposure (Pang 1973). Excavating sponges erode hard substratum through a combination of chemical dissolution and mechanical removal of $\mathrm{CaCO}_{3}$ fragments (chips, distinctly recognizable by their scalloped surface) (Cobb 1969; Rützler and Rieger 1973; Pomponi 1980). Previous efforts studying the contribution of the individual components suggest that the mechanical fraction generally accounts for the majority (up to 98\%) of total erosion (e.g. Rützler and Rieger 1973; Acker and Risk 1985; Nava and Carballo 2008; Fang et al. 2013b; Webb et al. 2017), although an opposite pattern has been described by Zundelevich et al. (2007) for Pione vastifica ( $\beta$-stage). The product of mechanical erosion by sponges contributes significantly to the total pool of fine silt-sized sediment on coral reefs (Carballo et al. 2017 and references therein), which is essential to reef cementation and stabilization (Ogden 1977).

There is ample scientific support that excavating sponges respond positively in both abundance and boring activity to factors such as deteriorating water quality (e.g. eutrophication), ocean acidification, elevated sea surface temperature and increased availability of substratum 
following coral mortality (Schönberg et al. 2017b and references therein). Nevertheless, they are rarely incorporated in standardized reef surveys and experimental work is restricted to a limited number of species. This is in part due the endolithic life-style of the sponge. The few surveys that do include excavating sponges, such as the ReefBudget method (Perry et al. 2012), have to rely on scarce and often inconsistent data with respect to rates of bioerosion. Currently, techniques to estimate these rates are inconsistent and the procedures involved are tedious and prone to methodological bias.

The available published data on total estimated sponge bioerosion displays tremendous variation with rates ranging from $0.3 \mathrm{~kg} \mathrm{~m}^{-2} \mathrm{y}^{-1}$ to $29.5 \mathrm{~kg} \mathrm{~m}^{-2} \mathrm{y}^{-1}$ (Schönberg et al. 2017a: S3 Table). Density of the invaded substratum has a considerable impact on the attained rates (e.g. Schönberg 2002b; Calcinai et al. 2007a), but also factors such as studied species, boring strategy, developmental stage ( $\alpha, \beta, \gamma$ or $\delta$-stage) and applied methodology can cause significant variability (Schönberg et al. 2017a: S3 Table). In early work, erosion rates were generally determined based on weight loss of the host substratum over a designated period (Neumann 1966; Rützler 1975; Bak 1976; Schönberg 2002b), occasionally in combination with chip collection experiments to quantify mechanical erosion. Buoyant weight has been commonly implemented to estimate coral growth rates, but has also proven a good proxy to quantify rates of sponge bioerosion (Schönberg 2002b; Fang et al. 2013a). A major advantage of the buoyant weight technique is that the studied material can remain submerged during the weighing process. This approach on itself, however, is not selective to means of erosion (i.e. chemical or mechanical), which is relevant, for instance, when studying the effect of ocean acidification on the chemical fraction of sponge bioerosion. Recent efforts have therefore implemented incubation techniques to study both components separately. By incubating sponge infested substratum it is possible to determine chemical bioerosion based on the increase in alkalinity of the incubation-water over time (Zundelevich et al. 2007). This technique has now been widely adopted to quantify effects of environmental change and future climate scenarios on rates of bioerosion (e.g. Fang et al. 2013b; Wisshak et al. 2013; Wisshak et al. 2014; Achlatis et al. 2017; Webb et al. 2017). A major disadvantage of this method, however, is that sponges are kept in a limited body of water for a relatively long time, which could strongly affect the metabolism of the sponge following food and oxygen depletion and the accumulation of waste products (Rix et al. 2017). Moreover, the lack of understanding of the processes underlying mechanical chip removal adds great uncertainty to previously quantified mechanical bioerosion rates. Indeed, periodic expelling of chips and/or contamination of filters by other material may induce error in the quantification of mechanical bioerosion (e.g. Fang et al. 2013b; Webb et al. 2017). Evidently, the considerable variation in published sponge bioerosion rates stresses the need for consistency in applied procedures.

In the present study, the chemical and mechanical bioerosion rate of six boring sponge species that dominate bioeroding community on the southern Caribbean reefs of Curaçao was determined. Chemical rates were estimated using flow-through incubation techniques, which were compared to previously used standing incubations. For mechanical rates a distinction was made between actual chips and other $\mathrm{CaCO}_{3}$ material removed by the eroding activities of the 
sponge over a designated time span. These approaches were then compared to conventional buoyant weighing procedures. We aim to optimize techniques to quantify bioerosion and to determine variation in chemical and mechanical bioerosion of sponge species with different functional traits (e.g. presence/absence of photosymbionts, boring strategies and developmental stages) and in light of a changing reef environment.

\section{Materials and methods}

\section{Study area and sample collection}

Sponges were collected on reefs at the leeward side of the island of Curaçao (southern Caribbean) in February 2017. Reefs here are characterized by a reef flat and a drop-off that gradually slopes down from approximately $10 \mathrm{~m}$ depth (Van Duyl 1985). Samples were collected at Snake Bay $\left(12^{\circ} 8^{\prime} \mathrm{N}, 68^{\circ} 59^{\prime} \mathrm{W}\right)$, Piscadera Bay $\left(12^{\circ} 7^{\prime} \mathrm{N}, 68^{\circ} 58^{\prime} \mathrm{W}\right)$ and Directors Bay $\left(12^{\circ} 3^{\prime} \mathrm{N}, 68^{\circ} 51^{\prime} \mathrm{W}\right)$ between 5 and $15 \mathrm{~m}$ depth. Sponge infested cores of the most dominant species were removed from old dead coral substratum using a pneumatic drill with hole-saw (inner diameter $45 \mathrm{~mm}$ ). Collected cores were cleaned and non-sponge organisms removed. The collected material was kept in large flow-through aquaria in the research facilities of the Carmabi Research Station. Cores were left to acclimatize and recover for at least seven days. Full tissue regeneration was observed within this period (see also Wisshak et al. 2012).

\section{Species description}

A total of six excavating sponge species were collected including two gallery-forming species (Figure 4.1): Cliona caribbaea (Carter 1882) and C. aprica (Pang 1973), three cavity-forming species: C. delitrix (Pang 1973), C. amplicavata (Rützler 1974) and Siphonodictyon brevitubulatum (Pang 1973) and Suberea flavolivescens, a species that formed a dense network through the carbonate substratum (from here onwards referred to as network-forming). This is, to our knowledge, the first scientific description of $C$. amplicavata and S. flavolivescens on the reefs of Curaçao. Both gallery-forming species belong to the Cliona viridis species complex (Schönberg 2002a; Zea and Weil 2003) and harbour high densities of dinoflagellate zooxanthellae (Pang 1973). C. delitrix has also been reported to hold zooxanthellae, but in considerably lower densities (Granados et al. 2008). The other three species do not appear to harbour symbiotic zooxanthellae (Pang 1973; Rützler 1974). $\alpha$-Stage specimens were collected for $C$. aprica, C. delitrix, C. amplicavata, and S. brevitubulatum and $\beta$-stage specimens for $C$. caribbaea and $S$. flavolivescens. The $\alpha$-stage of $C$. delitrix (often confused with $C$. laticavicola) (Chaves-Fonnegra et al. 2015) was chosen since the $\beta$-stage was only recently observed for the first time on Curaçaoan reefs (Mueller 2017). To allow for inter-specific comparison, all specimens, with the exception of $C$. amplicavata, were collected from infested dead Orbicella spp. coral, which is the most common substratum on many Caribbean reefs (Goreau 1959b; Bak 1977) and of average skeletal density $\left(\sim 1.8 \mathrm{~g} \mathrm{~cm}^{-3}\right.$ ) (Hughes 1987). C. amplicavata was only found in Acropora spp. rubble. Same sized uncolonized cores were collected from old Orbicella spp. and Acropora spp. skeleton to serve as control substratum throughout all experiments allowing adjustment for possible dissolution through micro-bioerosion, abrasion caused by 
handling and accretion due to the potential presence of calcifying organisms (e.g. Fang et al. 2012). In order to prevent decomposition of S. brevitubulatum, the majority of the soft internal tissue was removed and cores were left to recover for several additional days before being used experimentally. Species identification was confirmed by spicule morphology analysis.
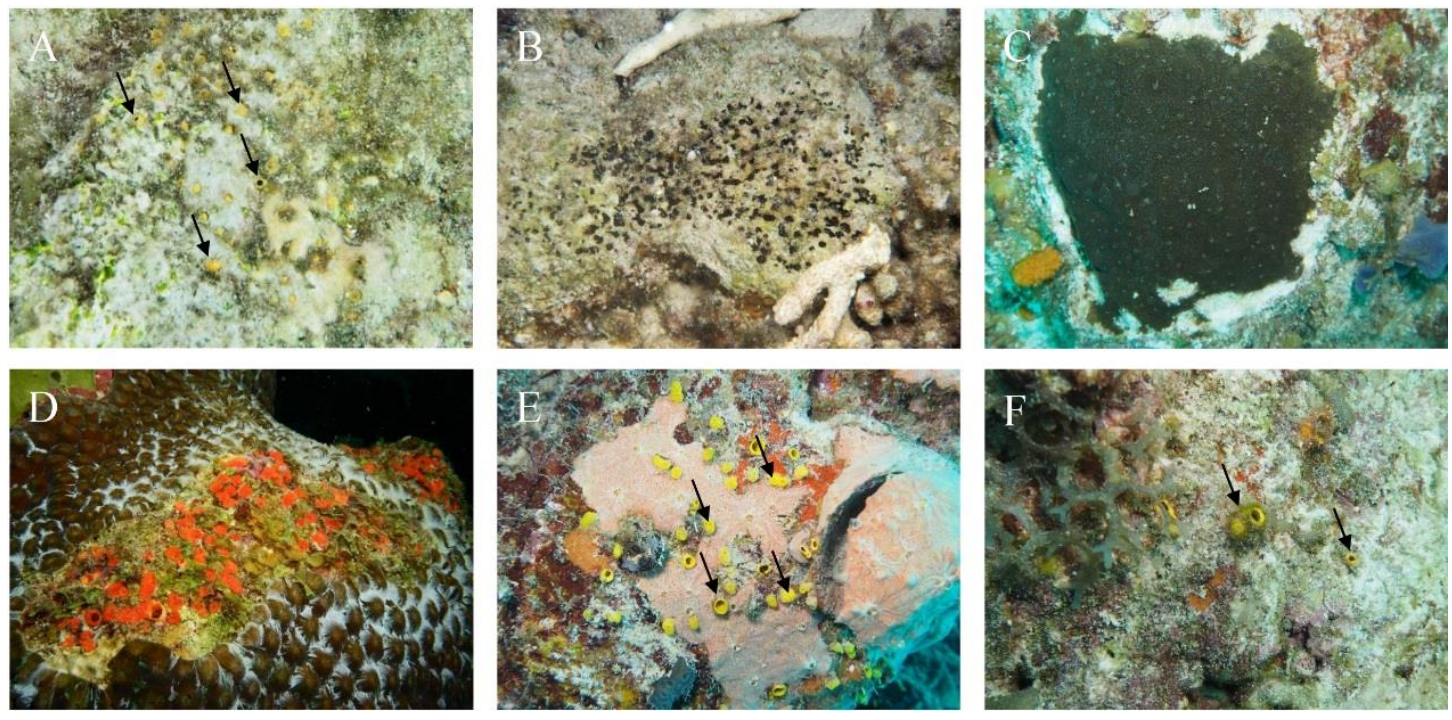

Figure 4.1. In situ close-up of the six studied excavating sponge species. A. Cliona amplicavata ( $\alpha$-stage) B. Cliona aprica ( $\alpha$-stage) C. Cliona caribbaea ( $\beta$-stage) $D$. Cliona delitrix ( $\alpha$-stage) $\boldsymbol{E}$. Siphonodictyon brevitubulatum ( $\alpha$-stage), please note that the yellow fistules of $S$. brevitubulatum are surrounded by a different sponge species (pink coloured) $\boldsymbol{F}$. Suberea flavolivescens ( $\alpha$-stage). Black arrows in $\boldsymbol{A}, \boldsymbol{E}$ and $\boldsymbol{F}$ point out oscula and ostia of the studied sponges.

\section{Experimental set-ups}

Sponge bioerosion was measured in different experimental settings in large flow-through $(\sim 5 \mathrm{~L}$ $\mathrm{min}^{-1}$ ) holding tanks. We used $500 \mathrm{~mL}$ incubation chambers consisting of a transparent body with detachable top and bottom. The top contained an electrically driven magnetic stirrer to ensure thorough homogenisation of the incubation water. The incubation chambers were used in a standing mode (ST) without water flowing through and in a continuous flow-through mode (FT). In addition and for comparison total bioerosion was also determined gravimetrically by applying the buoyant weight (BW) technique (Bak 1973; Davies 1989) to hanging cores. Debris from these cores was collected to determine the mechanical bioeroding capacity of the sponges.

With the conventional standing incubation the effect of incubation time on rates of chemical erosion was tested for two sponge species. C. caribbaea (symbiont-rich) and C. delitrix (symbiont-poor) infested cores were incubated for 1, 2, 4 and $6 \mathrm{~h}$ in fully enclosed incubation chambers. Two cores were placed in each chamber which was sealed air-tight to avoid gaseous exchange with the external environment. Chambers were largely submerged to prevent 
temperature anomalies. Temperature $\left({ }^{\circ} \mathrm{C}\right)$ and oxygen $(\%)$ were monitored continuously over the course of the incubation using a PreSens $\mathrm{O}_{2}$ sensor (Fibox 4, PSt3). Incubations at night were carried out for a maximum of 3 hours to avoid oxygen levels declining too much $(>80 \%$ loss of $\mathrm{O}_{2}$ saturated water). Samples for alkalinity $(\sim 250 \mathrm{~mL})$ and nutrient $\left(\mathrm{PO}_{4}{ }^{3-}, \mathrm{NH}_{4}{ }^{+}, \mathrm{NO}_{3}{ }^{-}\right.$, $\mathrm{NO}_{2}^{-}$) analyses were collected at the start and the end of each run.

For the flow-through set-up the incubation chamber was modified to allow a constant through flow of water (Figure 4.2). Each chamber contained two sponge-infested or control cores. By means of a peristaltic pump, with adjustable flow-speed, fresh sea water was transported from a reservoir into the chamber where the magnetic stirrer ensured homogenized mixing. The excess water overflowed through flexible Teflon ${ }^{\circledR}$ tubing into a collection vessel. A filter $(0.7$ $\mu \mathrm{m}$ pore size) was placed on the out-flow tube to prevent loss of particulate material from the chamber. A flow-speed of $1.5 \mathrm{~L} \mathrm{~h}^{-1}$ allowed for continuous refreshment of the incubation water approximately every 20 minutes while still obtaining a reliable signal for water chemistry analyses. This flow-through incubation method ensured a constant supply of food and prevented excessive build-up of waste products, therewith simulating more adequately in situ reef conditions. Water samples for alkalinity $\left(\mathrm{A}_{\mathrm{T}}, \mu \mathrm{mol} \mathrm{kg} \mathrm{kg}^{-1}\right)$ and nutrient analyses were collected at the inlet and outlet of the incubation chamber. The system was run for 30 minutes before sample collection started, to allow for an initial full refreshment of the incubation water. This was done to prevent catching a signal that might be affected by the initial incubation water the cores were placed in. Additionally, a possible effect of sponge handling and subsequent acclimatization was herewith largely excluded. Final dissolution rates were calculated from the observed difference in $\mathrm{A}_{\mathrm{T}}$ which was corrected for the retention time of the water in the incubation chamber. Cores were carefully removed from the FT chamber immediately after water sample collection. Subsequently, all particulate material produced during the entire duration of the incubation $(\sim 1 \mathrm{~h})$ was collected as a measure for mechanically removed substratum (mechanical erosion). Chemical and mechanical erosion of sponge infested and control cores was determined at day and night in the FT incubation because erosion rates of sponges, particularly symbiontbearing species, were found to be affected by light availability (diurnal variation) (Hill 1996; Fang et al. 2016; Webb et al. 2017). Flow through incubations were run for all six sponge species. For two of them, C. caribbaea and C. delitrix, results (chemical dissolution rates) of the FT were compared to results of the ST incubation. In order to test the consistency in time of the FT methodology, additional water samples were collected for C. caribbaea 1, 2, 3 and $4 \mathrm{~h}$ after the start of the incubation while the FT system kept running.

Mechanical bioerosion by the six species was also determined over a longer period (7 consecutive days) as part of the buoyant weight approach. Three infested cores per species and blank cores were positioned upside down directly above glass funnels $(\varnothing 10 \mathrm{~cm})$ in a large holding tank. Particulate material caught in each funnel (placed in a collection vial) as well as in the FT incubation chamber was collected on pre-combusted $\left(550^{\circ} \mathrm{C}, 3 \mathrm{~h}\right)$, pre-weighed, 0.7 $\mu \mathrm{m}$ Whatman ${ }^{\circledR}$ glass microfiber filters. Filters were subsequently re-combusted $\left(550^{\circ} \mathrm{C}, 3 \mathrm{~h}\right)$ to remove any organic material and re-weighed resulting in an estimate of the particulate material produced over the course of each experimental approach. As an additional analysis, material 
collected on the filters of the one week experiment was homogenized and subsampled. Scanning Electron Microscopy (SEM) of the subsample was used to estimate the actual contribution of sponge chips, non-chip $\mathrm{CaCO}_{3}$ fragments and other material (e.g. spicules, foreign materials). FT filters contained too little material for adequate SEM analysis. Results of both experiments were corrected for the weight of fragments collected from uncolonized control cores to ensure that only the fraction removed by the sponge would be included in estimates of total mechanical erosion.

To determine total bioerosion based on the loss in buoyant mass, the hanging cores were first weighed at the beginning of the 7-day collection period following the BW technique as described by Fang et al. (2013b). Cores were re-weighed after hanging for three full weeks. All measurements were conducted by means of an electronic weighing apparatus ( $0.1 \mathrm{mg}$ accuracy). Organic sponge tissue was assumed to equal the density of the ambient sea water so to eliminate the effect of sponge growth. Buoyant weight of the cores was calibrated for fluctuations in seawater density. The change in weight of each core over the three week period was corrected by the change in buoyant mass of the uncolonized control cores and subsequently converted into rates of total sponge bioerosion.

\section{Chemical erosion}

The mass of $\mathrm{CaCO}_{3}$ dissolved by each sponge $(\Delta M)$ was calculated from the change in total alkalinity over the course of the incubation following Equation 4.1 (Zundelevich et al. 2007; Nava and Carballo 2008). Fluctuations in $\mathrm{PO}_{4}{ }^{3-}, \mathrm{NH}_{4}{ }^{+}, \mathrm{NO}_{3}{ }^{-}$and $\mathrm{NO}_{2}{ }^{-}$can affect $\Delta \mathrm{A}_{\mathrm{T}}$ and are therefore included in the equation as well (Jacques and Pilson 1980; Wisshak et al. 2013).

$\Delta M_{(\mathrm{CaCO})}=0.5\left(\mathrm{~mol} \mathrm{eq}^{-1}\right) \times\left[\Delta \mathrm{A}_{\mathrm{T}}+\Delta \mathrm{PO}_{4}-\Delta \mathrm{NH}_{4}+\Delta\left(\mathrm{NO}_{3}+\mathrm{NO}_{2}\right)\right] \times \mathrm{V}_{\mathrm{SW}} \times \rho_{\mathrm{sw}} \times 100$

where $\Delta \mathrm{A}_{\mathrm{T}}$ is the change in total alkalinity. Multiplication factor ' 0.5 ' reflects the $2: 1$ relationship between the increase in $\mathrm{A}_{\mathrm{T}}$ during $\mathrm{CaCO}_{3}$ dissolution. $\mathrm{V}_{\mathrm{SW}}$ is the volume (L) of seawater in the incubation chamber and $\rho_{\mathrm{Sw}}$ is seawater density $\left(1.023 \mathrm{~kg} \mathrm{~L}^{-1}\right)$. The multiplier ' 100 ' incorporates the molecular mass of $\mathrm{CaCO}_{3}$. 


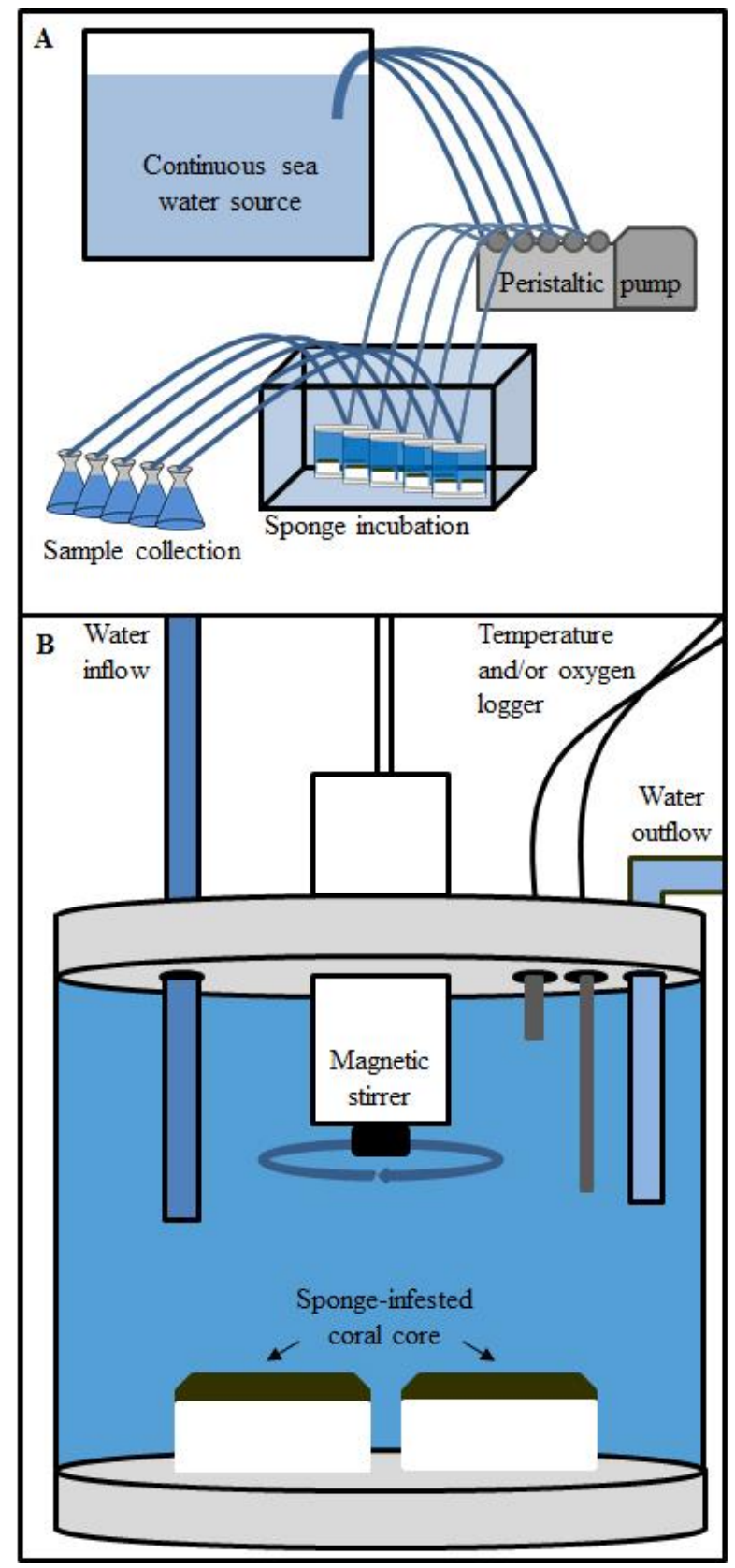

Figure 4.2. Flow-through set-up. A. Overview of the flow-through incubation set-up including the peristaltic pump with adjustable flow-speed continuously pumping fresh sea water through the incubation chambers. The excess water subsequently overflows into collection vessels for nutrient and total alkalinity analyses. Chambers are partly submerged in a holding tank to prevent temperature fluctuation. B. Close-up of a flow-through incubation chamber $(500 \mathrm{~mL})$ holding two sponge infested limestone cores. 
$\mathrm{A}_{\mathrm{T}}$ was measured using an Automated Spectrophotometric Alkalinity System (ASAS) (Liu et al. 2015) following the method outlined by Breland and Byrne (1993) and Yao and Byrne (1998). This optical titration procedure has a remarkable high precision $\left( \pm 0.7 \mu \mathrm{mol} \mathrm{\textrm {kg } ^ { - 1 }}\right)$ making it possible to detect minor alkalinity fluctuations. $A_{T}$ was measured immediately after sample collection. To compensate for a drift in $\mathrm{A}_{\mathrm{T}}$ over the course of the experimental period, certified reference material (CRM; supplied by Dr. A. Dickson, Scripps Institution of Oceanography) was analyzed every 20 samples. Filtered (acrodisc: $0.2 \mu \mathrm{m}$ pore size) nutrient samples were stored at $-20^{\circ} \mathrm{C}$ until transport to the Royal Netherlands Institute for Sea Research (NIOZ) where they were analysed on a QuAAtro continuous flow analyzer (SEAL Analytical, $\mathrm{GmbH}$, Norderstedt, Germany) following GO-SHIP protocol (Hydes et al. 2010). Final chemical erosion rates were expressed in $\mathrm{mg} \mathrm{CaCO}_{3} \mathrm{~cm}^{-2} \mathrm{~d}^{-1}$ or convertible units and nutrient dynamics in $\mu \mathrm{mol} \mathrm{L}^{-1} \mathrm{~h}^{-1}$.

\section{Determining sponge surface area and biomass}

At the end of each experiment all cores were photographed to estimate the surface area covered by external sponge tissue. For $\alpha$-stage (only single inhalant and exhalant papillae are visible on the surface) sponges, the area in between the outermost papillae was taken as a measure for infested surface area. For $\beta$-stage specimens (papillae are fused into an encrusting sheet) the total sponge covered surface on both the top and side of each core was used. Additionally, the cores used in the BW experiment were dissolved in acid $(2 \mathrm{M} \mathrm{HCl})$ after the final weighing to isolate the sponge tissue for wet and dry-weight $\left(24 \mathrm{~h}\right.$ at $\left.60^{\circ} \mathrm{C}\right)$ measurements and to verify the absence of other macroborers (e.g. worms and molluscs).

\section{Statistics}

All statistical testing was conducted in the R programming environment R 3.3.2 (R Core Team 2013) using the packages "stats" and "gam". Assumptions of normality and homogeny were checked visually and all collected data were fourth-root transformed to stabilize variances. Generalized additive modelling was implemented to predict the fluctuations in rates of chemical erosion as a function of time in ST incubations. Two-way ANOVA (species and diurnal cycle) testing complemented by post-hoc Tukey-HSD paired comparisons were used to examine variation in patterns of excavation among and within the six studied sponge species.

\section{Results}

\section{Chemical erosion}

Comparison of chemical dissolution rates acquired through ST and FT incubation showed that duration of the ST incubation had a distinct effect on sponge chemical bioerosion. Dissolution rates by $C$. caribbaea $(F=33.9, p<0.001)$ and $C$. delitrix $(F=27.3, p<0.001)$ declined significantly as a function of the time cores were retained in the confined water body of the incubation chamber (Figure 4.3). After 2 hours $\left(\mathrm{T}_{2}\right)$, rates had already decreased by $31 \%$ ( $C$. caribbaea) and $70 \%$ (C. delitrix) compared to the modelled rate at $\mathrm{T}_{0}$, which is the hypothetical point at which the sponge should not yet experience any negative effect of being incubated. Comparison of dissolution rates between the ST and the FT during day-time (Figure 4.3) shows 
that the chemical dissolution extrapolated to $\mathrm{T}_{0}$ of the ST incubation, C. caribbaea: $0.014 \mathrm{mg}$ $\mathrm{cm}^{-2} \mathrm{~h}^{-1}$ (95\% CI: 0.011 - 0.017) and C. delitrix: $0.021 \mathrm{mg} \mathrm{cm}^{-2} \mathrm{~h}^{-1}$ (95\% CI: 0.012 - 0.032), agrees with dissolution values obtained in FT incubation (Table 4.1, daily rates). A negative effect of incubation time was not observed for the two species in the flow-through methodology, at least not up to $4 \mathrm{~h}$ after the start of the incubation (Figure 4.3). This supports the long-term consistency of the FT methodology introduced here. Consequently it was decided to focus on measurements with the FT set-up.

Chemical dissolution rates acquired through FT incubation revealed significant $(F=4.11, p<$ 0.01) variation among sponge species (Figure $4.4 \&$ Table 4.1). Interspecific variation was predominantly associated with morphology resulting in significantly slower erosion rates for both gallery-forming species: $C$. caribbaea and $C$. aprica. These high density zooxanthellate sponges also showed clear diurnal disparity with significantly higher ( $p<0.01$ for both species) dissolution rates during the day (Figure 4.4). In C. amplicavata, C. delitrix, S. brevitubulatum and $S$. flavolivescens night erosion appeared to be higher than day erosion, although this was not significant for any of these species. Notably, the inflow water was slightly more basic during day-time $(\mathrm{pH}=8.08 \pm 0.001)$ compared to night-time $(\mathrm{pH}=8.02 \pm 0.007)$.

\section{Nutrient dynamics}

Considerable nutrient accumulation (especially $\mathrm{NH}_{4}{ }^{+}$and $\mathrm{NO}_{3}{ }^{-}$) occurred at day-time in ST (1 h) incubations holding C. caribbaea (Table 4.2). Accumulation of nutrients after 1 hour was largely prevented in the FT system (Table 4.2). Observed fluctuations in phosphate concentrations were largely negligible, there was essentially no indication for net uptake. In FT incubations a minor elevation in levels of ammonium and nitrate was observed during most incubations at night. During the day, these nutrients appear to be consumed in the majority of the incubations, including those containing non-infested cores. Nitrogen can be taken up by the sponge itself or by its zooxanthellae (i.e. C. caribbea, C. aprica and to a lesser extend $C$. delitrix). The observed decline in control incubations (containing uncolonized cores) indicates that there is likely also an effect on nutrient levels caused by micro-organisms in the incubation water. When considering the nutrient trajectories in the control water many of the observed dynamics in chambers containing sponges are largely dampened (Table 4.2).

\section{Mechanical erosion}

The mechanical fraction of sponge bioerosion was based on the weight of the particulate material collected in the incubation chamber after $1 \mathrm{~h}$ FT incubation and in the funnels for the long-term (7 day) collection experiment, yielding two estimates per species (Figure 4.5 \& Table 4.1). Although both estimates are in a similar range, rates derived from FT incubation are consistently higher. SEM analyses of 7-day filters did revealed that, depending on the species, $17 \%$ to $45 \%$ of the collected material (long-term) could be attributed to actual sponge chips (Figure 4.6). The remaining material consisted mostly of non-chip $\mathrm{CaCO}_{3}$ fragments with some occurrences of foreign planktonic material or spicules. The fraction of non-chip material that was dislodged by sponge could not be traced back directly to boring activity and thus represents an estimate (i.e. total non-chip $\mathrm{CaCO}_{3}$ fragments minus the fragment production by non-infested cores). $\mathrm{CaCO}_{3}$ fragment removal differed significantly among species $(F=4.43, p=0.003)$, 


\section{Chapter IV}

with most material removed by $C$. delitrix and C. amplicavata. Similar to the chemical fraction, there appears to be a strong diurnal pattern in mechanical substrate removal in both symbiontrich species as well as the symbiont-poor $C$. delitrix (Table 4.1). ANOVA testing of the FT results showed that mechanical erosion at night was significantly lower only for $C$. caribbaea ( $F=59.6, p=0.016)$. It should be noted, however, that only one sample was available for $C$. aprica at day which strongly hampered proper statistical testing. Mechanical erosion by the other four species did not differ significantly at day or night $(p>0.05)$.

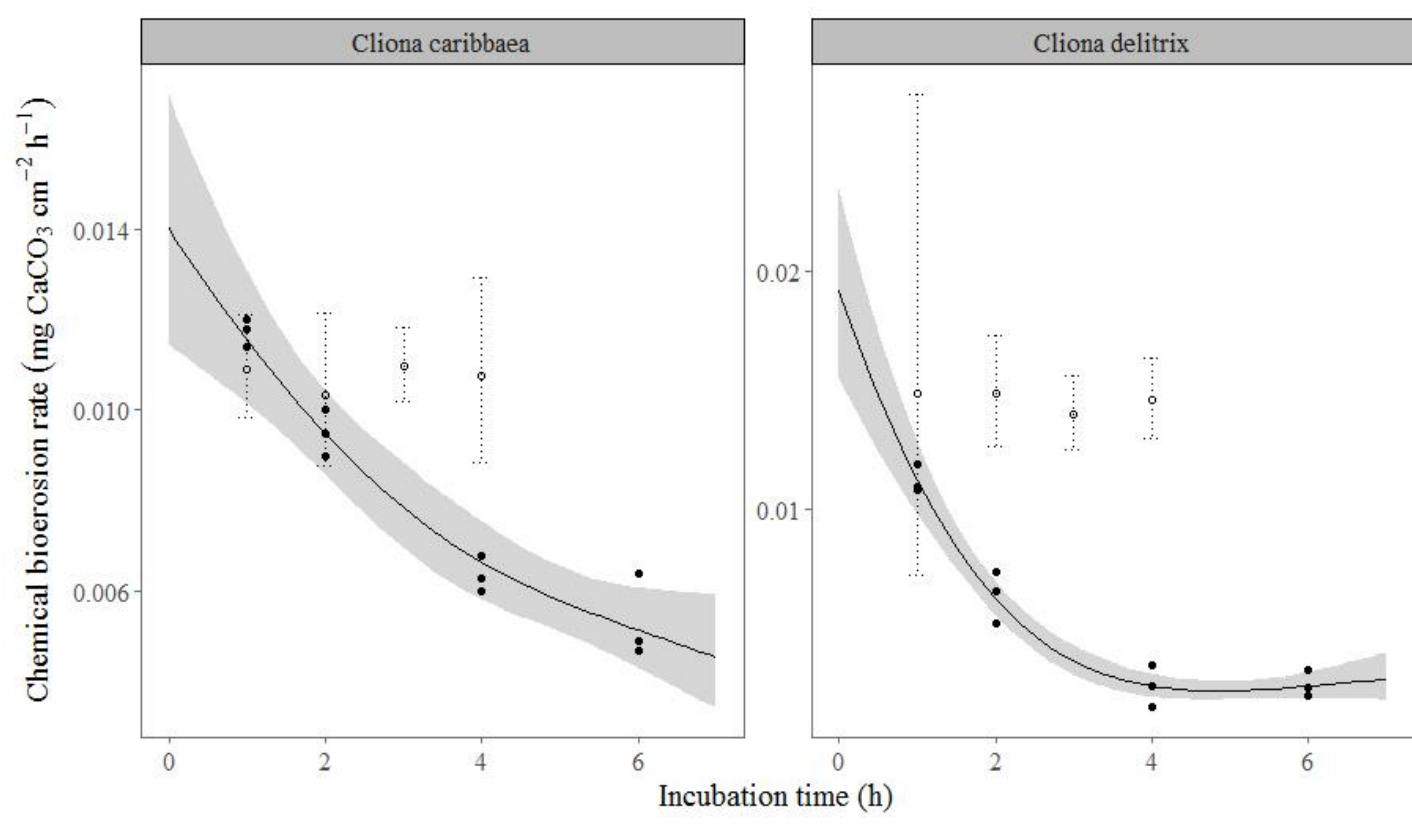

Figure 4.3. Modelled effect of incubation time on sponge chemical bioerosion rates. Black

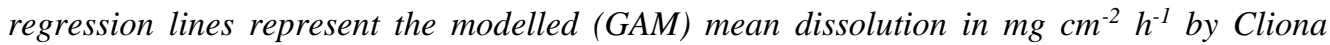
caribbaea (left panel) and Cliona delitrix (right panel) in standing incubations. $95 \%$ confidence limits are indicated by the grey band. Solid dots show the calculated mean dissolution rates at $T_{1}, T_{2}, T_{4}$ and $T_{6}(S T)$. Open dots represent rates derived from running flow-through incubations at $T_{1}, T_{2}, T_{3}$ and $T_{4}$, dotted lines here indicate 95\% confidence limits. All rates are obtained using alkalinity anomaly techniques. 


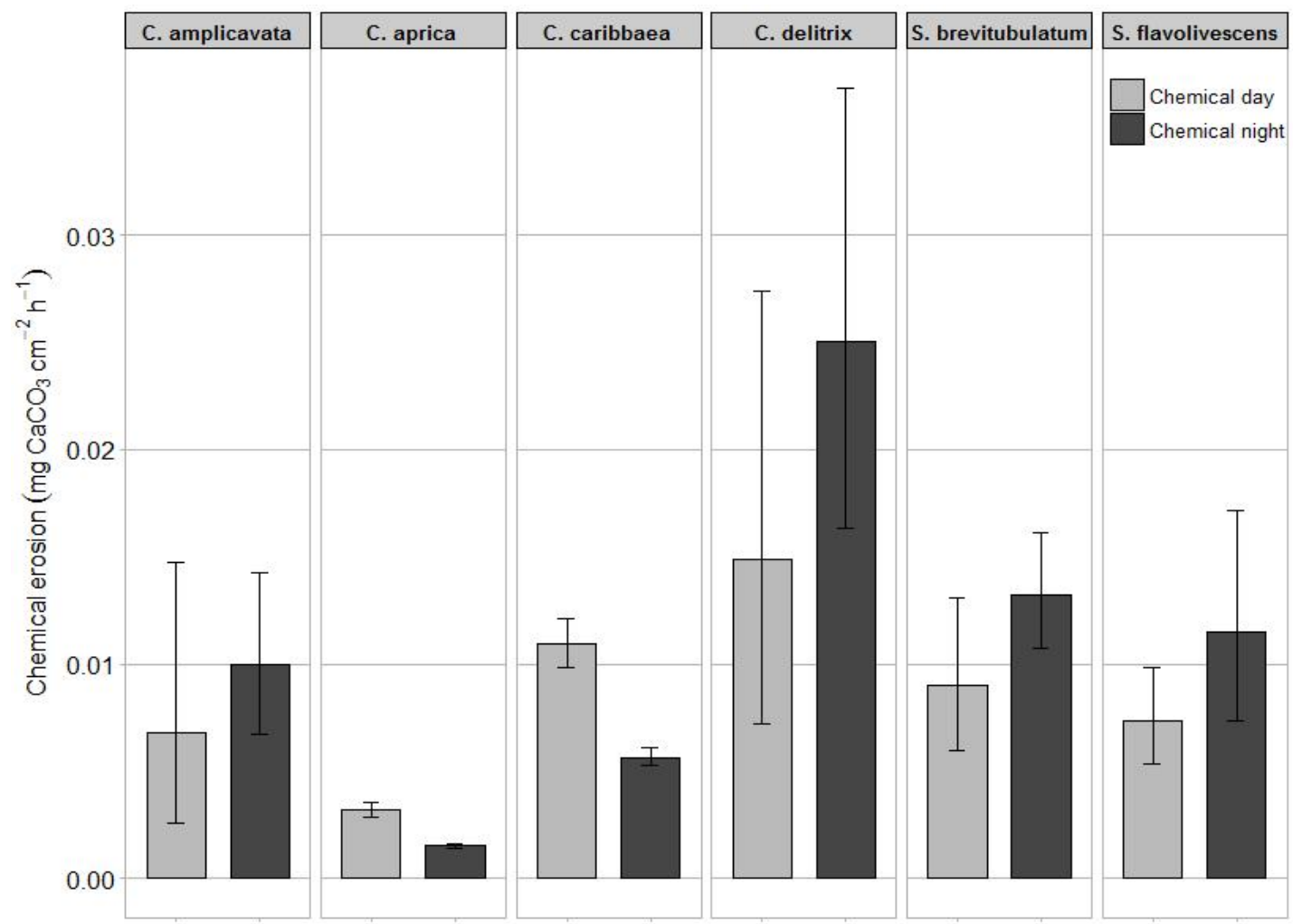

Figure 4.4. Chemical bioerosion. Hourly rates of chemical dissolution ( $\left.\mathrm{mg} \mathrm{CaCO}_{3} \mathrm{~cm}^{-2} \mathrm{~d}^{-1}\right)$ are given for the six studied sponge species (top). Both day (light grey) and night (dark grey) erosion rates are included. Rates are derived from alkalinity change in flow-through incubation. Error bars indicate the $95 \%$ confidence limits.

Table 4.1. Summary of all determined sponge bioerosion rates. Bioerosion rates of six excavating sponge species acquired through different methodologies: flow-through incubation; buoyant weight; long-term ( 7 day) collection of mechanically removed material. The different elements (chemical and mechanical erosion both at day and night) that make up the total bioerosion are, when available, individually presented per method. Day and night rates are given for $12 \mathrm{~h}$ day and night. Erosion rates given in $\mathrm{mg} \mathrm{CaCO} \mathrm{cm}^{-2} \mathrm{~d}^{-1}$, with the exception of annual rates in the final column (given in $\mathrm{kg} \mathrm{CaCO}_{3} \mathrm{~m}^{-2} \mathrm{y}^{-1}$ ). Buoyant weight acquired rates converted to sponge dry weight ( $g$ ) are given separately (BM) in $\mathrm{mg}$ (daily). 


\begin{tabular}{|c|c|c|c|c|c|}
\hline Species & Method & $\begin{array}{l}\text { Chemical } \\
\text { day }(12 \text { h })\end{array}$ & $\begin{array}{c}\text { Mechanical } \\
\text { day }(12 \text { h) }\end{array}$ & $\begin{array}{c}\text { Chemical } \\
\text { night }(12 \text { h) }\end{array}$ & $\begin{array}{l}\text { Mechanical } \\
\text { night }(12 \text { h) }\end{array}$ \\
\hline \multirow[t]{4}{*}{ C. amplicavata } & Flow-through & $\begin{array}{c}0.08 \\
(0.03-0.18)\end{array}$ & $\begin{array}{c}1.54 \\
(1.01-2.25)\end{array}$ & $\begin{array}{c}0.12 \\
(0.08-0.17)\end{array}$ & $\begin{array}{c}1.26 \\
(0.93-1.67)\end{array}$ \\
\hline & Buoyant weight & & & & \\
\hline & BM (g DWsponge) & & & & \\
\hline & 7-day collection & & & & \\
\hline \multirow[t]{4}{*}{ C. aprica } & Flow-through & $\begin{array}{c}0.04 \\
(0.03-0.04)\end{array}$ & $\begin{array}{l}0.85 \\
\text { (NA) }\end{array}$ & $\begin{array}{c}0.02 \\
(0.02-0.02)\end{array}$ & $\begin{array}{c}0.45 \\
(0.33-0.60)\end{array}$ \\
\hline & Buoyant weight & & & & \\
\hline & BM (g DWsponge) & & & & \\
\hline & 7-day collection & & & & \\
\hline \multirow[t]{4}{*}{ C. caribbaea } & Flow-through & $\begin{array}{c}0.13 \\
(0.12-0.15)\end{array}$ & $\begin{array}{c}1.62 \\
(1.32-1.96)\end{array}$ & $\begin{array}{c}0.07 \\
(0.06-0.07)\end{array}$ & $\begin{array}{c}0.44 \\
(0.74-1.50)\end{array}$ \\
\hline & Buoyant weight & & & & \\
\hline & BM (g DWsponge) & & & & \\
\hline & 7-day collection & & & & \\
\hline \multirow[t]{4}{*}{ C. delitrix } & Flow-through & $\begin{array}{c}0.18 \\
(0.09-0.33)\end{array}$ & $\begin{array}{c}2.08 \\
(1.12-3.56)\end{array}$ & $\begin{array}{c}0.30 \\
(0.20-0.44)\end{array}$ & $\begin{array}{c}0.87 \\
(0.81-0.94)\end{array}$ \\
\hline & Buoyant weight & & & & \\
\hline & BM (g DWsponge) & & & & \\
\hline & 7-day collection & & & & \\
\hline \multirow[t]{4}{*}{ S. brevitubulatum } & Flow-through & $\begin{array}{c}0.11 \\
(0.07-0.16)\end{array}$ & $\begin{array}{c}0.69 \\
(0.18-1.54)\end{array}$ & $\begin{array}{c}0.16 \\
(0.13-0.19)\end{array}$ & $\begin{array}{c}1.02 \\
(0.40-2.20)\end{array}$ \\
\hline & Buoyant weight & & & & \\
\hline & BM (g DWsponge) & & & & \\
\hline & 7-day collection & & & & \\
\hline \multirow[t]{4}{*}{ S. flavolivescens } & Flow-through & $\begin{array}{c}0.09 \\
(0.06-0.12)\end{array}$ & $\begin{array}{c}0.26 \\
(0.14-0.44)\end{array}$ & $\begin{array}{c}0.14 \\
(0.09-0.21)\end{array}$ & $\begin{array}{c}0.29 \\
(0.18-0.45)\end{array}$ \\
\hline & Buoyant weight & & & & \\
\hline & BM (g DWsponge) & & & & \\
\hline & 7-day collection & & & & \\
\hline
\end{tabular}


Quantification of bioerosion rates of six Caribbean excavating sponge species

Table 4.1. continued.

\begin{tabular}{|c|c|c|c|c|c|}
\hline Species & Method & $\begin{array}{c}\text { Total } \\
\text { chemical } \\
(24 \mathrm{~h})\end{array}$ & $\begin{array}{c}\text { Total mechanical } \\
\qquad(24 \mathrm{~h})\end{array}$ & $\begin{array}{l}\text { Total erosion } \\
\text { (daily) }\end{array}$ & $\begin{array}{l}\text { Total erosion } \\
\text { (annual) }\end{array}$ \\
\hline \multirow[t]{4}{*}{ C. amplicavata } & Flow-through & 0.20 & 2.80 & 3.00 & 10.95 \\
\hline & Buoyant weight & & & $\begin{array}{c}1.90 \\
(0.93-3.49)\end{array}$ & $\begin{array}{c}6.94 \\
(3.40-12.74)\end{array}$ \\
\hline & BM (g DWsponge) & & & $\begin{array}{c}76.18 \\
(16.44-230.2)\end{array}$ & 0.028 \\
\hline & 7-day collection & & $\begin{array}{c}2.52(1.70-3.60) \\
{[0.77]}\end{array}$ & & \\
\hline \multirow[t]{4}{*}{ C. aprica } & Flow-through & 0.06 & 1.30 & 1.36 & 4.96 \\
\hline & Buoyant weight & & & $\begin{array}{c}0.71 \\
(0.51-0.96)\end{array}$ & $\begin{array}{c}2.59 \\
(1.86-3.50)\end{array}$ \\
\hline & BM (g DWsponge) & & & $\begin{array}{c}66.40 \\
(33.33-119.5)\end{array}$ & 0.024 \\
\hline & 7-day collection & & $\begin{array}{c}0.47(0.37-0.60) \\
{[0.30]}\end{array}$ & & \\
\hline \multirow[t]{4}{*}{ C. caribbaea } & Flow-through & 0.20 & 2.06 & 2.26 & 8.25 \\
\hline & Buoyant weight & & & $\begin{array}{c}0.29 \\
(0.01-0.23)\end{array}$ & $\begin{array}{c}1.06(0.04- \\
1.84)\end{array}$ \\
\hline & BM (g DWsponge) & & & $\begin{array}{c}21.99 \\
(18.95-25.39)\end{array}$ & 0.008 \\
\hline & 7-day collection & & $\begin{array}{c}0.39(0.30-0.52) \\
{[0.29]}\end{array}$ & & \\
\hline \multirow[t]{4}{*}{ C. delitrix } & Flow-through & 0.48 & 2.95 & 3.43 & 12.52 \\
\hline & Buoyant weight & & & $\begin{array}{c}2.30 \\
(1.09-4.31)\end{array}$ & $\begin{array}{c}8.40 \\
(3.98-15.73)\end{array}$ \\
\hline & BM (g DWsponge) & & & $\begin{array}{c}272.4 \\
(95.98-622.5)\end{array}$ & 0.099 \\
\hline & 7-day collection & & $\begin{array}{c}1.79(1.16-2.67) \\
{[0.50]}\end{array}$ & & \\
\hline \multirow[t]{4}{*}{ S. brevitubulatum } & Flow-through & 0.27 & 1.71 & 1.98 & 7.23 \\
\hline & Buoyant weight & & & $\begin{array}{c}0.94 \\
(0.39-1.94)\end{array}$ & $\begin{array}{c}3.43 \\
(1.42-7.08)\end{array}$ \\
\hline & BM (g DWsponge) & & & $\begin{array}{c}195.0 \\
(53.75-516.1)\end{array}$ & 0.014 \\
\hline & 7-day collection & & $\begin{array}{c}0.69(0.44-1.06) \\
{[0.26]}\end{array}$ & & \\
\hline \multirow[t]{4}{*}{ S. flavolivescens } & Flow-through & 0.23 & 0.55 & 1.01 & 2.01 \\
\hline & Buoyant weight & & & $\begin{array}{c}0.17 \\
(0.01-0.27)\end{array}$ & $\begin{array}{c}0.62 \\
(0.04-0.99)\end{array}$ \\
\hline & BM (g DWsponge) & & & $\begin{array}{c}7.00 \\
(4.26-10.83)\end{array}$ & 0.003 \\
\hline & 7-day collection & & $\begin{array}{c}0.38(0.29-0.50) \\
{[0.10]}\end{array}$ & & \\
\hline
\end{tabular}


Table 4.2. Summary of nutrient dynamics in the incubation chambers. Change in nutrient $\left(\mathrm{NH}_{4}^{+}, \mathrm{NO}_{2}^{-}, \mathrm{NO}_{3}^{-}, \mathrm{PO}_{4}{ }^{3+}\right)$ concentration ( $\mu \mathrm{mol} \mathrm{L}$ sea water ${ }^{-1}$ ) after one hour of flow-through (FT) incubation. Concentrations are given for all six species and uncolonized control cores at day and night. Nutrient dynamics are also provided for $C$. caribbaea in 1 h standing incubation (ST). Rates are given with their standard deviation. $n$ : number of nutrient samples. No standard deviation is given (NA) when only 1 nutrient value was available.

\begin{tabular}{|c|c|c|c|c|c|c|}
\hline Species & & $\mathbf{n}$ & $\mathrm{NH}_{4}{ }^{+}$ & $\mathrm{NO}_{2}^{-}$ & $\mathrm{NO}_{3}{ }^{-}$ & $\mathrm{PO}_{4}{ }^{3+}$ \\
\hline \multirow{2}{*}{ C. caribbaea $(\mathrm{ST})$} & Day & 3 & $2.06 \pm 0.49$ & $0.04 \pm 0.03$ & $0.62 \pm 0.43$ & $0.04 \pm 0.03$ \\
\hline & Night & 2 & $0.87 \pm 0.39$ & $0.04 \pm 0.01$ & $0.2 \pm 0.01$ & $0.02 \pm 0.004$ \\
\hline \multirow[t]{2}{*}{ C. caribbaea (FT) } & Day & 6 & $-2.41 \pm 4.26$ & $-0.04 \pm 0.04$ & $-1.92 \pm 2.91$ & $0.01 \pm 0.01$ \\
\hline & Night & 2 & $0.98 \pm 0.19$ & $0.03 \pm 0.00$ & $0.42 \pm 0.02$ & $0.07 \pm 0.01$ \\
\hline \multirow[t]{2}{*}{ C. amplicavata (FT) } & Day & 4 & $0.39 \pm 0.14$ & $0.02 \pm 0.02$ & $0.12 \pm 0.05$ & $0.01 \pm 0.01$ \\
\hline & Night & 3 & $0.11 \pm 0.21$ & $-0.01 \pm 0.01$ & $0.21 \pm 0.28$ & $0.00 \pm 0.00$ \\
\hline \multirow[t]{2}{*}{ C. aprica (FT) } & Day & 3 & $-0.15 \pm 0.01$ & $-0.02 \pm 0.03$ & $-0.04 \pm 0.03$ & $0.01 \pm 0.00$ \\
\hline & Night & 2 & $0.36 \pm \mathrm{NA}$ & $-0.003 \pm \mathrm{NA}$ & $0.16 \pm \mathrm{NA}$ & $0.03 \pm 0.04$ \\
\hline \multirow[t]{2}{*}{ C. delitrix (FT) } & Day & 5 & $-0.21 \pm 0.41$ & $-0.03 \pm 0.02$ & $-0.37 \pm 0.39$ & $0.03 \pm 0.05$ \\
\hline & Night & 3 & $0.12 \pm 0.17$ & $-0.03 \pm 0.06$ & $0.25 \pm 0.07$ & $-0.01 \pm 0.02$ \\
\hline \multirow[t]{2}{*}{ S. brevitubulatum (FT) } & Day & 2 & $0.03 \pm \mathrm{NA}$ & $-0.11 \pm \mathrm{NA}$ & $-0.04 \pm \mathrm{NA}$ & $0.01 \pm 0.03$ \\
\hline & Night & 1 & $0.05 \pm \mathrm{NA}$ & $-0.07 \pm \mathrm{NA}$ & $0.14 \pm \mathrm{NA}$ & $0.06 \pm \mathrm{NA}$ \\
\hline \multirow[t]{2}{*}{ S. flavolivescens (FT) } & Day & 3 & $-0.06 \pm 0.25$ & $-0.06 \pm 0.03$ & $0.78 \pm N A$ & $0.06 \pm 0.02$ \\
\hline & Night & 2 & $-0.31 \pm 0.13$ & $0.03 \pm 0.003$ & $1.35 \pm 0.04$ & $0.06 \pm 0.00$ \\
\hline \multirow[t]{2}{*}{ Uncolonized core (FT) } & Day & 1 & $-0.22 \pm \mathrm{NA}$ & $-0.01 \pm \mathrm{NA}$ & $-0.16 \pm$ NA & $-0.01 \pm \mathrm{NA}$ \\
\hline & Night & 1 & $0.03 \pm \mathrm{NA}$ & $-0.04 \pm \mathrm{NA}$ & $-0.03 \pm \mathrm{NA}$ & $-0.01 \pm \mathrm{NA}$ \\
\hline
\end{tabular}

\section{Total bioerosion}

Total bioerosion (i.e. chemical and mechanical) quantified from FT incubations was consistently higher than BW acquired rates (Table 4.1 \& Figure 4.5). For C. amplicavata, $S$. brevitubulatum and $S$. flavolivescens both rates are still within a similar range. However, the discrepancy between methods was particularly pronounced in both symbiont-rich species $(C$. caribbaea and $C$. aprica) and $S$. flavolivescens. Nevertheless, the observed patterns of interspecific variation were highly comparable among both approaches, with highest erosion rates found for the three cavity-forming species. Lowest rates are consistently observed for networkforming S. flavolivescens. There appears to be considerable variation in BW rates among cores of the same species (suggested by the large confidence intervals), in particular among the cavity-

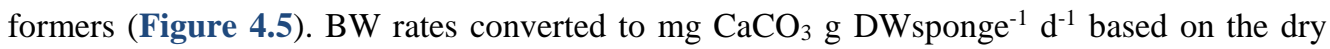
weight of the sponge tissue in each core yielded considerable similarity in patterns of interspecific variation to rates based on surface area (Table 4.1 \& Appendix 4.A). The cavityforming $C$. amplicavata, however, appear to be an exception to this apparent surface area biomass relation. FT estimates indicate that for five out of the six species the mechanical fraction accounted for the majority of total bioerosion $(86 \%-96 \%)$. Interestingly, for the network forming S. flavolivescens chemical (46\%) and mechanical (54\%) contributed almost equally. 


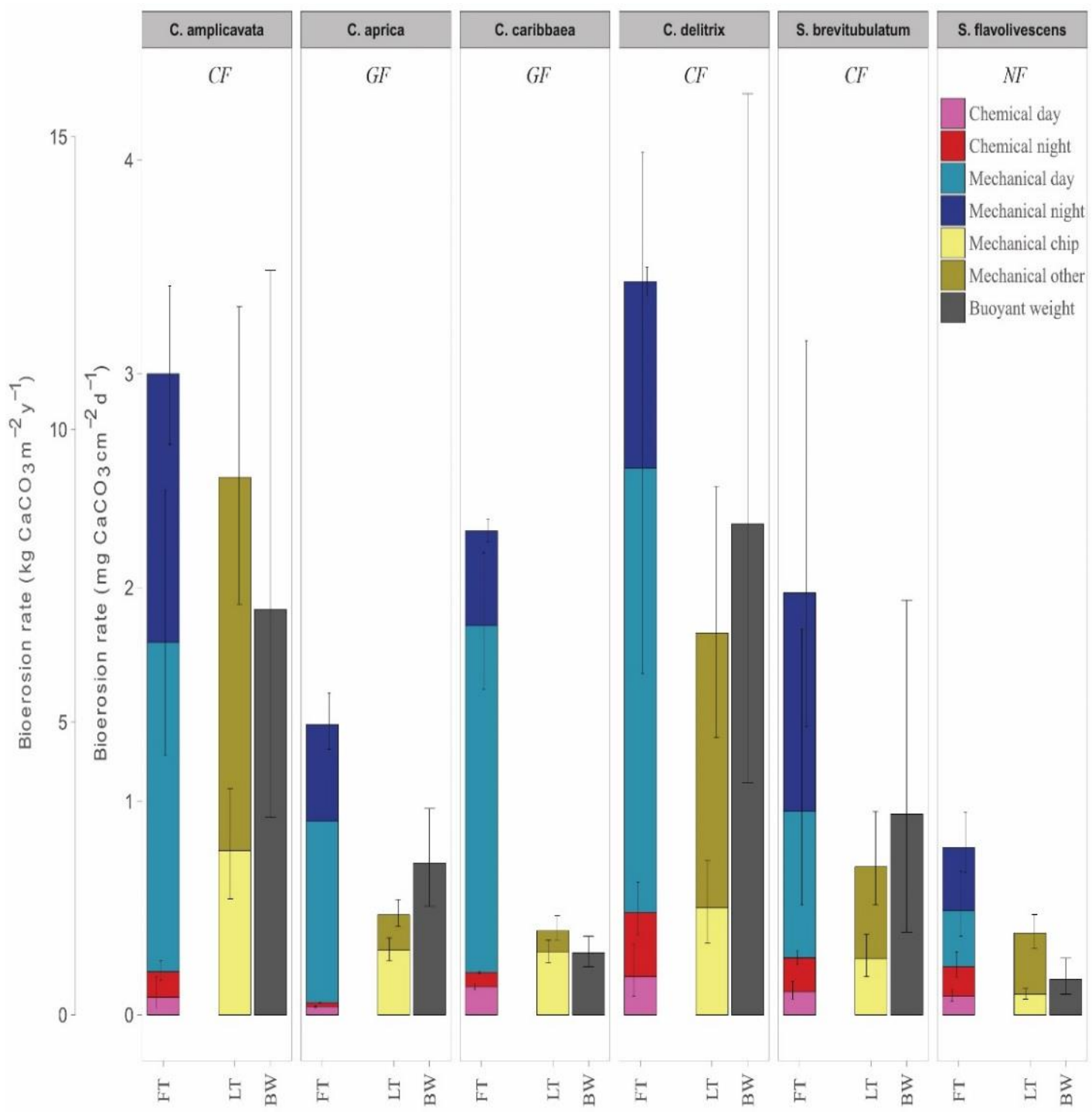

Figure 4.5. Total sponge bioerosion. Final rates of total erosion by the six sponge species (top) acquired through flow-through incubation (FT) and buoyant weight loss over a three week period $(B W)$. The different bioerosive components $(F T)$ are based on a 12 h day/night diurnal cycle. Mechanical bioerosion rates based on long-term collection are provided separately (LT). Here, a distinction is made between actual sponge chips and other $\mathrm{CaCO}_{3}$ fragments that have been dislodged through sponge bioeroding activity. Rates are given in $\mathrm{mg} \mathrm{CaCO}_{3} \mathrm{~cm}^{-2} d^{-1}$ and $\mathrm{kg} \mathrm{CaCO} 3 \mathrm{~m}^{-2} y^{-1}$. Error bars indicate the $95 \%$ confidence intervals for each individual element. Species and boring strategies are provided at the top of each panel, CF: cavity-forming, GF: gallery-forming; NF: network-forming. 


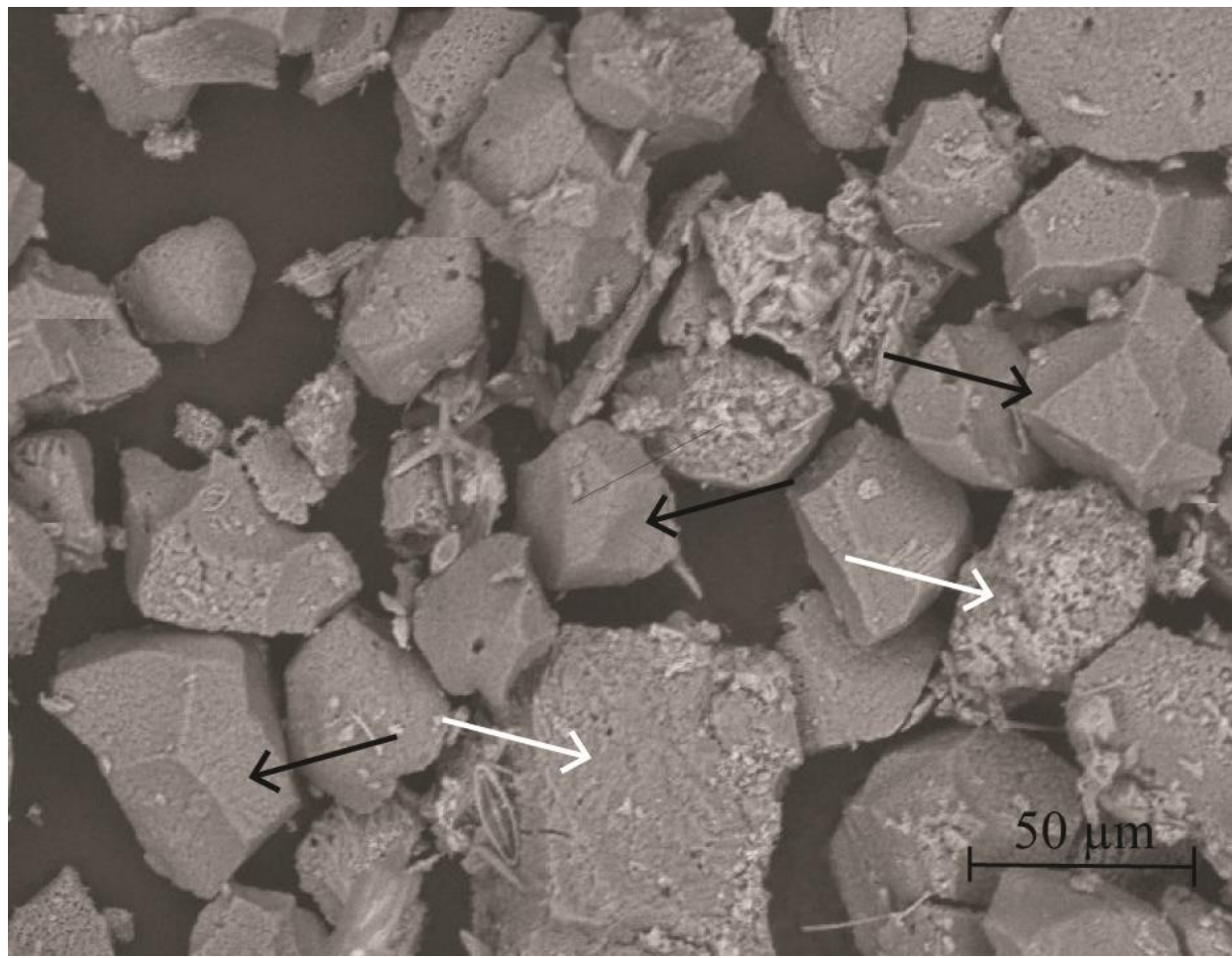

Figure 4.6. Scanning Electron Microscopy (SEM) image of material produced by $C$. caribbaea. The provided image shows only a fraction of the total material produced over the 7day collection experiment. Black arrows: some characteristic sponge chips, white arrows: other $\mathrm{CaCO}_{3}$ fragments. Scale is provided in lower-right corner.

\section{Discussion}

We quantified the bioerosive capacity of six excavating sponge species commonly found on the reefs of Curaçao. This is the first time individual chemical and mechanical rates and total bioerosion rates are provided simultaneously for the majority of these species. Overall, Cliona delitrix and Cliona amplicavata (both cavity-forming species) were found to have the highest boring rates. $C$. delitrix is indeed known to be among the most destructive bioeroding sponges on many Caribbean reefs (Chaves-Fonnegra and Zea 2007). It should be pointed out that $C$. amplicavata was found solely in Acropora spp. rubble which is of higher skeletal density than Orbicella spp. (Hughes 1987). Substratum density can strongly affect bioerosion, where denser substratum generally leads to higher erosion rates (e.g. Bak 1976; Schönberg 2002b). Erosion by $C$. caribbaea and $C$. aprica, both adopting a gallery-forming strategy, was found to be considerably lower compared to cavity-formers. Nonetheless, these species, together with $C$. delitrix, are the dominant excavating sponges on the shallow reefs of Curaçao and many sites in wider Caribbean region (Perry et al. 2012; Chaves-Fonnegra et al. 2015; Mueller 2017) and thus contribute significantly to total macrobioerosion. S. flavolivescens, previously undescribed to occur on Curaçaoan reefs, appears to be the weakest eroder of all sponge species studied here. 
This network-forming species adapts a different boring strategy (i.e. network-forming) from those outlined by Murphy et al. (2016).

\section{Methodological considerations}

Comparison of the chemical bioerosion in the flow-through (FT) and standing (ST) approach reveals that an incubation time of 1 hour or longer in the ST incubation chambers was too long to estimate the purported "real" bioerosion rate. Bioerosion steeply dropped in time, suggesting that food depletion and or waste accumulation inhibited chemical bioerosion. Neumann (1966) suggests that sponges can bore more actively in a high water flow environment, possibly due to a larger supply of fresh sea water. Being known as highly efficient filter-feeding organisms (e.g. Vacelet and Boury-Esnault 1995) it is not surprising that these sponge have the capacity to rapidly consume the available food sources, both particulate and dissolved (Mueller et al. 2014) in any limited body of water. Consequential stress could significantly affect the metabolism of the sponge, possibly redirecting energy away from secondary processes such as boring and focussing more on vital functions like maintaining a high pumping velocity. Extrapolation of rates determined in the $\mathrm{ST}$ to $\mathrm{T}_{0}$ revealed chemical dissolution rates comparable to rates found in the FT incubators. This implies that whenever the ST set-up is preferable, the optimal incubation times should be determined in advance in the devices used and individually for each sponge species. To date, standing incubation techniques generally resulted in lower rates for chemical erosion, but have also predominantly been used to test the effect of environmental change on the boring efficiency through sea water manipulation (Schönberg et al. 2017a and references therein). Although in most of these studies the authors looked at relative variation in rates as a response to treatment, the acquired rates should be interpreted with some degree of caution. The processes acting inside these enclosed chambers are largely unknown and unpredictable. More desirable, therefore, would be the use of FT incubation, since here the effect of incubation time on measured variables appears to be negligible (Figure 4.3 \& Table 4.2).

$\mathrm{CaCO}_{3}$ fragment production (including sponge chips) in the FT incubation chambers was generally higher than the fragment production by hanging cores in a large aquarium. Webb et al. (2017) propose that elevated levels of $\mathrm{Ca}^{2+}$ in incubation water (ST) following chemical dissolution of $\mathrm{CaCO}_{3}$ may cause contraction of the conductive pathway of the sponge. Such a contraction could result in an initial pulse ejection of the chips present within the sponge tissue, resulting in excessively high production over the relatively short incubation. However, in our FT incubations accumulation of $\mathrm{Ca}^{2+}$ will have been limited because the incubation water was constantly refreshed. Since the discrepancy between methods was most pronounced in both symbiont-rich species ( $C$. caribbaea and $C$. aprica) and $S$. flavolivescens, it is more likely that the reversed orientation of the hanging cores may have reduced the boring capacity of these sponges following limited light availability. In addition, this methodology (hanging cores) might be more prone to loss of a fraction of the produced silt-sized fragments due to water movement in the holding tank (Fang et al. 2013b). Overall, there is still hardly any information available on the mechanisms underlying mechanical erosion or the expelling of dislodged material by excavating sponges. Both approaches (short and long term collection), however, can 
provide better insight in the mechanisms underlying mechanical erosion and the expelling of dislodged material by excavating sponges.

For most species, final FT rates are comparable to rates acquired gravimetrically based on the loss in buoyant weight (BW) of the invaded cores (Figure 4.5). Strongest variation between methodologies was found in C. caribbaea and C. aprica and S. flavolivescens. Similar to the long-term fragment collection, the relatively low BW estimates are likely the result of the reversed orientation of the cores (see previous paragraph). This might also explain why for some hanging species mechanical erosion exceeded total bioerosion. $\mathrm{CaCO}_{3}$ fragment production was only measured in the first week. Possibly the boring capacity of the hanging sponges had further decreased in the two following weeks. We therefore argue that BW estimates in this study may reflect a slight underestimation of sponge bioeroding capacity and FT obtained rates should be considered more reliable. Up to now conflicting studies generally found up to five times higher rates obtained through buoyant weighing compared to standing incubation derived estimates (Fang et al. 2013b; Webb et al. 2017). The observed discrepancy has caused scepticism regarding the use of BW technique. Our results, however, support the use of the buoyant weight methodology, as described in previous studies, as a reliable approach to estimate rates of bioerosion and suggest that the previous implementation of standing incubation may often have resulted in underestimated rates. Nevertheless, to examine the effect that changing environmental conditions might have on bioerosion it is essential to consider both fractions independently. Particularly in view of the effect factors such as elevated levels of $\mathrm{CO}_{2}$ in ocean water have on the chemical dissolution capacity of the various sponge species. Preferably this is done in an incubation set-up where chemical erosion can be determined individually as well. Consequently, the methods presented here, with some minor improvements where necessary (read BW, long-term fragment collection), can and should be used concurrently depending on the underlying scientific aim.

\section{Bioerosion by different species}

FT incubation revealed that the chemical bioerosive capacity of $C$. caribbaea and C. aprica was notably higher during the day (Figure 4.4). This is likely related to the presence of photosynthetic symbionts in these viridis-complex species. The photosynthetic capacity of intracellular symbiotic zooxanthellae was pinpointed as a possible primary source of carbon for these sponge species (Hill 1996; Weisz et al. 2010; Fang et al. 2014). A consequential diurnal variation in rates of bioerosion following light availability has previously been described for $C$. caribbaea in St. Eustatius (Lesser Antilles) (Webb et al. 2017), Cliona varians in the Florida Keys (Hill 1996) and Cliona orientalis in Australia (Schönberg 2006; Fang et al. 2016), all of which are species in the $C$. viridis species complex. Symbiodinium spp. in $C$. delitrix, present in much lower densities (Granados et al. 2008), did not seem to cause significant variation in chemical rates between day and night. Accordingly, this species appears to rely predominantly on heterotrophic feeding to fuel bioerosion. Similarly, no significant diurnal pattern was found for any of the other species, although chemical dissolution by these species does appear to be somewhat lower during the day. Possibly, these species benefited from the small decrease in sea water $\mathrm{pH}$ at night. Indeed, photosynthesis during the day-time and respiration at night by 
primary producers is known to cause diurnal variation in $\mathrm{pH}$ (Smith et al. 2013). The latter suggests that these sponges might respond more firmly than symbiont-rich species to a decreasing sea water $\mathrm{pH}$ as a result of ocean acidification. This corresponds to the findings of Webb et al. (2017) that at night dissolution by C. caribbaea only started increasing at a strongly elevated $\mathrm{pCO}_{2}$ of the incubation water. The absence of a clear diurnal pattern in azooxanthellate species is in compliance with findings of Schönberg (2008) who studied rates of Cliona celata in the North Sea, but also with Zundelevich et al. (2007) who studied the zooxanthellate Pione vastifica in the Red Sea.

Rates obtained through FT incubation show that mechanical removal of invaded substratum contributed most significantly $(86-95 \%)$ to total estimated bioerosion rates. Only for $S$. flavolivescens (network-forming) chemical and mechanical erosion was approximately equal. A detailed visual inspection of $S$. flavolivescens specimens, however, revealed a large quantity of $\mathrm{CaCO}_{3}$ particles to be present in its dense tissue network. It is possible that this species retains most of the mechanically removed fragments, causing an underestimation of mechanical erosion when using methods as presented here. With the exception of $S$. flavolivescens the ratio between dissolution and mechanical substrate removal resembles that described in the majority of the published literature, where it is suggested that mechanical dissolution contributes between $83 \%$ to $98 \%$ to total bioerosion by various studied excavating sponge species (e.g. Rützler and Rieger 1973; Acker and Risk 1985; Nava and Carballo 2008; Fang et al. 2013b; Webb et al. 2017). Based on our findings there appears to be no obvious variation in this ratio among species adapting different boring strategies. However, we did observe some diurnal variation in the mech:chem ratio, with chemical erosion having a slightly more pronounced contribution to total erosion at night. This was especially clear for $C$. delitrix (chemical contribution: day: $8 \%$, night $26 \%$ ) and might be a result of decreased $\mathrm{pH}$ at night-time. This suggests that the mech:chem ration might shift with the predicted ocean acidification scenarios. The portion of mechanically removed fragments that could be attributed to the characteristic sponge chips was limited compared to the total collected $\mathrm{CaCO}_{3}$ for all six species. This was particularly evident in the three cavity-forming species (C. amplicavata $C$. delitrix and $S$. brevitubulatum) and $S$. flavolivescens, where chips accounted for a mere $17 \%-24 \% . \mathrm{CaCO}_{3}$ material produced by $C$. aprica $(74 \%)$ and C. caribbaea (64\%) contained considerably more sponge chips, suggesting chip production may be linked to the adopted excavating strategy. Although chips are with great certainty removed by the sponge itself, many of the remaining $\mathrm{CaCO}_{3}$ fragments are most likely also the dislodged as a result of sponge boring activity since substantially less of this material was present on filters of uncolonized cores.

The sum of chemical and mechanical rates (both FT) realized final estimates of total annual bioerosion for all six studies species: $10.95 \mathrm{~kg} \mathrm{~m}^{-2} \mathrm{y}^{-1}$ (C. amplicavata); $4.96 \mathrm{~kg} \mathrm{~m}^{-2} \mathrm{y}^{-1}$ (C. aprica); $8.25 \mathrm{~kg} \mathrm{~m}^{-2} \mathrm{y}^{-1}$ (C. caribbaea); $12.52 \mathrm{~kg} \mathrm{~m}^{-2} \mathrm{y}^{-1}$ (C. delitrix); $7.23 \mathrm{~kg} \mathrm{~m}^{-2} \mathrm{y}^{-1}(S$. brevitubulatum) and $2.01 \mathrm{~kg} \mathrm{~m}^{-2} \mathrm{y}^{-1}$ (S. flavolivescens). With the exception of $C$. amplicavata all rates presented here are based on bioerosion of Orbicella spp. coral substratum which is of 'average' density and the most ubiquitous substratum on many reefs throughout the Caribbean. The considerable variation in published bioerosion rates (Schönberg et al. 2017a: S3 Table) can 
largely be attributed to factors such as the density of the substratum (e.g. Schönberg 2002b; Calcinai et al. 2007b), but also studied species, developmental stage and applied methodology. Acker and Risk (1985) provided total erosion rates for C. caribbaea in Orbicella spp. (8.0 kg $\left.\mathrm{m}^{-2} \mathrm{y}^{-1}\right)$ that are comparable to rates presented here. More recently, a considerably lower rate $\left(1.7 \mathrm{~kg} \mathrm{~m}^{-2} \mathrm{y}^{-1}\right)$ for total erosion by $C$. caribbaea was described for C. caribbaea, but in less dense Diploria spp. substratum and based on $6 \mathrm{~h}$ standing incubation (Webb et al. 2017). Similar rates $\left(2.2-2.5 \mathrm{~kg} \mathrm{~m}^{-2} \mathrm{y}^{-1}\right)$ were described for its 'Pacific equivalent' $C$. orientalis in massive Porites spp. coral (Wisshak et al. 2012; Fang et al. 2013b). Both Fang et al. (2013b) and Webb et al. (2017), however, found 3 to 5 times higher rates using the buoyant weight technique which results in rates that are similar to our findings. Estimates described for $C$. aprica range higher $\left(7.0 \mathrm{~kg} \mathrm{~m}^{-2} \mathrm{y}^{-1}\right)$ than our findings but it should be noted that this is based on erosion of extremely dense Conch shells (Rützler 1975). Substantially higher bioerosion rates were found for other C. viridis-complex species in substratum that is substantially denser than that of Orbicella spp. skeleton: $C$. orientalis (up to $17.6 \mathrm{~kg} \mathrm{~m}^{-2} \mathrm{y}^{-1}$ ) (Schönberg 2002b) and $C$. varians (up to $22.8 \mathrm{~kg}$ $\mathrm{m}^{-2} \mathrm{y}^{-1}$ ) (Hill 1996). Of the sponges studied here, C. delitrix is generally considered among the most aggressive eroding species (Rose and Risk 1985; Chaves-Fonnegra and Zea 2007) and our data appears to support this claim. The observed eroding capacity of $C$. delitrix in the $\alpha$-stage (this study) infers the bioeroding potential of $C$. delitrix since the $\beta$-stage is deemed considerably more destructive (Chaves-Fonnegra and Zea 2011). Next to $C$. delitrix, substantially high rates were only found for $C$. amplicavata, but in Acropora spp. rubble which has a considerably denser skeletal structure than Orbicella spp. Murphy et al. (2016) is the only study to have assessed the bioerosive capacity of $S$. brevitubulatum, adapting a completely different approach including substrate density and lateral expansion rate. Following their analogy a hypothetical sponge would bore with a rate of $4.4 \mathrm{~kg} \mathrm{~m}^{-2} \mathrm{y}^{-1}$ in carbonate substrate with a density of $1.7 \mathrm{~g}$ $\mathrm{cm}^{-3}$. This is in range with our estimates in substratum of comparable density. It is noteworthy that the estimated rates for $S$. brevitubulatum as presented in this work may not fully reflect the boring capacity of this species considering that most of the soft internal tissue needed to be removed to prevent tissue decomposition. To our knowledge, the bioeroding capacity of $C$. amplicavata has not previously been determined. Also, no representative data is available for $S$. flavolivescens or any species that adapts a similar excavating strategy.

Overall, patterns of inter-specific variation were strikingly similar when related to either surface area or biomass of the sponge. This would suggest a consistent link between surface area and internal biomass of the sponge with regards to bioerosive activity, which is in contrast to the few existing preceding studies addressing this issue (Rützler 1975; Holmes et al. 2009; Schönberg et al. 2017a). Nonetheless, it remains difficult to properly relate surface area to internal biomass of the sponge and we only quantified sponge biomass in three cores per species. Elaborate research on this specific relationship is crucial to properly estimate the contribution of excavating sponges to the reef carbonate budget.

We provide a comprehensive comparison of methods to quantify chemical and mechanical erosion and tentatively endorse methodologies that could be implemented widely in an attempt to support standardized data collection. The 'novel' FT technique described here is assumed to 
more adequately mimic natural in situ conditions, thus providing a more reliable representation of the bioerosive capacity of the excavating sponge community. We therefore encourage the use of flow-through incubation techniques next to buoyant weighing techniques, in particular when the aim is to determine the chemical fraction of bioerosion separately. If for some reason standing incubation techniques are preferred, the length of the incubation should be minimized or at least two time points should be included so that the signal at $\mathrm{T}_{0}$ can be predicted. It should be noted that determining mechanical erosion based on collected particulate material will always be prone to underestimation because loss of particles in the smallest size class cannot be fully excluded. This is the first study to estimate rates for such an extensive range of species encompassing the relevant majority of the sponge bioeroding community of Curaçao and the wider Caribbean. This includes multiple $\alpha$-stage species which, to date, have largely been ignored because their distinct papillate-morphology complicates linking assessed rates to the infested surface area (Schönberg et al. 2017a). Although we recognize that these data cover only part of the possible variation in rates of bioerosion caused by inter-specific and intra-specific variation, developmental stage, successional stage, density of the invaded substrate, reef-zone (Bak 1976) and demography it can be considered an important addition to the currently still limited knowledge on the bioerosive impact of the excavating sponge community on coral reefs. The data presented here, combined with previously published and future work can also be included in standardized surveys, such as the ReefBudget methodology (Perry et al. 2012), which would result in a more complete and correct image of the carbonate budget of Caribbean coral reefs. 


\section{Acknowledgement}

The authors would like to extend their sincere gratitude to Dr. Sven Zea for helping to identify the collected sponge species. We also wish to thank Karel Bakker and Sharyn Ossebaar for analysing nutrient samples, the Carmabi foundation for facilitating the fieldwork on Curaçao and the EMBC Master Programme. External funding for A.E. Webb and S.M.A.C. van Heuven was provided by the Netherlands Organization for Scientific Research (NWO grants 858.14.021 and 858.14.022). All research was conducted under research permit (\#2012/48584) issued by the Curaçaoan Ministry of Health, Environment and Nature (GMN) to the CARMABI Foundation. 


\section{Appendix 4.A}

Daily removed $\mathrm{CaCO}_{3}(\mathrm{mg})$ per gram of dry weight sponge tissue. Provided rates are based on the loss in buoyant weight of sponge infested cores ( $n=3$ per species) over a three week period. Sponge tissue was dried for $24 \mathrm{~h}$ at $60^{\circ} \mathrm{C}$. Rates are given for all six studied species: Cliona amplicavata (cavity-forming), Cliona aprica (gallery-forming), Cliona caribbaea (galleryforming), Cliona delitrix (cavity-forming), Siphonodictyon brevitubulatum (cavity-forming) and Suberea flavolivescens (network-forming). All rates include a correction for the outcomes of uncolonized control core experiments. Error bars represent the $95 \%$ confidence limits.

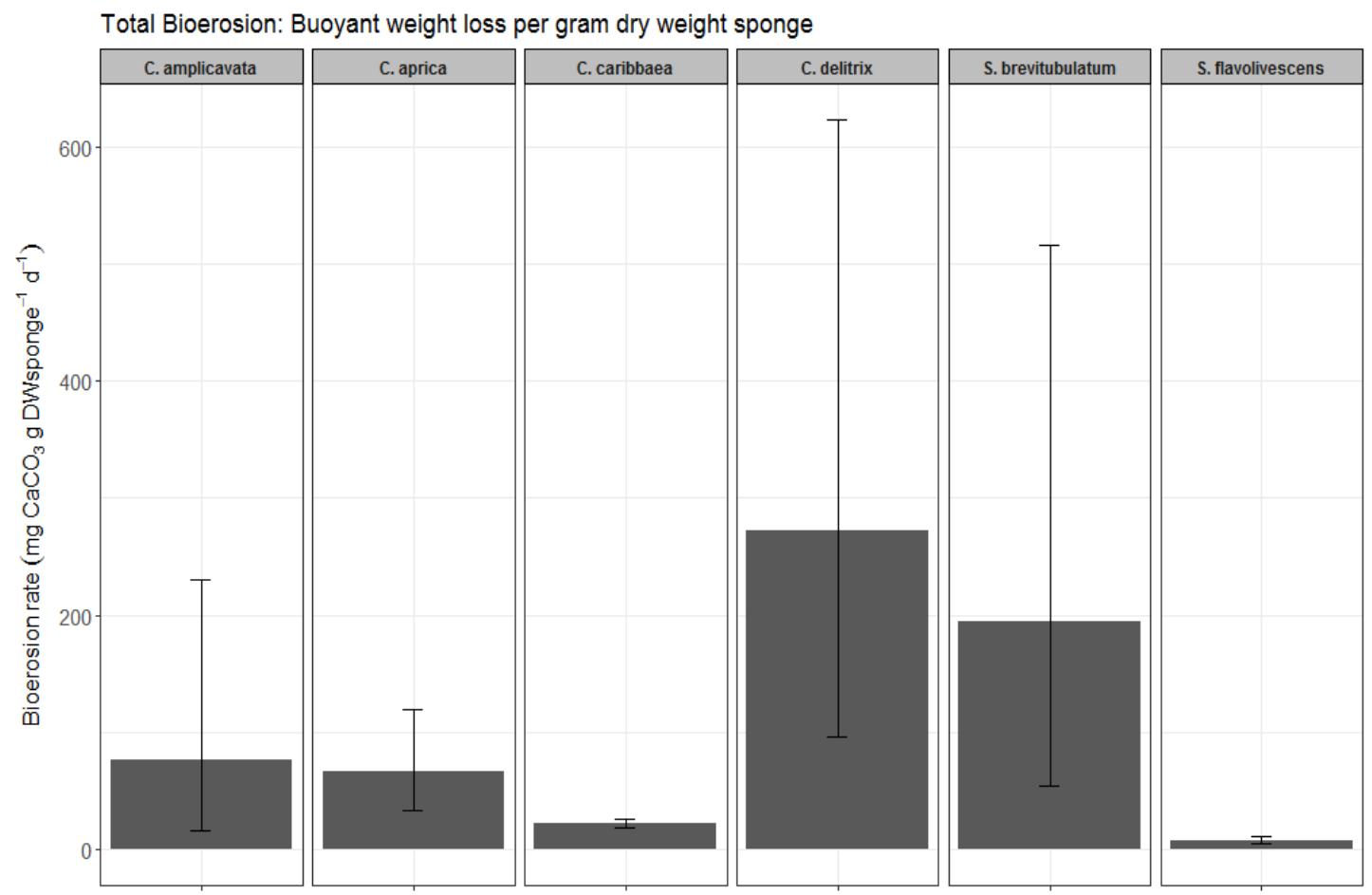

\section{Appendix 4.B}

Overview of the data supporting the results presented in this article. The overview table can be accessed through the following link:

https://journals.plos.org/plosone/article?id=10.1371/journal.pone.0197824\#sec018 
Chapter IV 


\section{8}

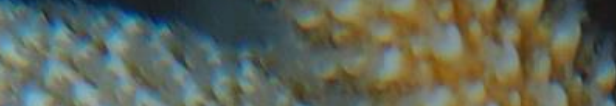

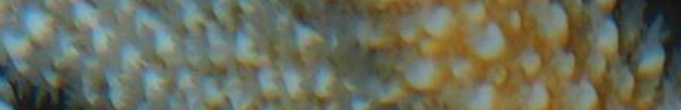

mentres

$$
\text { whores }
$$




\title{
CHAPTER V \\ Spatial variation in coral reef accretion potential poses significant concern for low-lying tropical coastlines
}

Didier M. de Bakker, Fleur C. van Duyl and Erik H. Meesters

Under revision at Global Change Biology

\begin{abstract}
Structural complexity provided by the calcium carbonate skeleton of corals and other calcifying organisms forms a vital natural barrier protecting low-lying shorelines from erosion and inundation. Recent shifts in coral reef community composition, however, strongly hamper the capacity of many reefs worldwide to maintain net carbonate accretion. We estimated, based on gross bioaccretion and bioerosion rates, the accretion potential of the shallow reef zone (at approximately $5 \mathrm{~m}$ and $10 \mathrm{~m}$ ) along the entire leeward coast of Bonaire (115 reef sites), an island with reefs that are thought to be in a better ecological condition than many sites elsewhere in the Caribbean region. Indeed, some regions, in particular the no-dive zone, still harbour structurally complex reefs with high net carbonate production $\left(+10 \mathrm{~kg} \mathrm{CaCO}_{3} \mathrm{~m}^{-2}\right.$ year $\left.^{-1}\right)$. Most sites, however, maintained a marginal production $\left(<2 \mathrm{~kg} \mathrm{CaCO}_{3} \mathrm{~m}^{-2}\right.$ year $\left.{ }^{-1}\right)$ or were in a state of net erosion, indicating that even reefs thought to be in relatively good condition are currently struggling to sustain net vertical accretion. The incapacity of these reefs to keep up with current projections of climate change induced future sea-level rise, poses a critical threat to the adjacent coastal region. Alarmingly, these low productivity and low complexity reefs are found especially in front of inundation-sensitive low-lying areas, including the most densely populated region around the capital of Bonaire (Kralendijk). Here, periodic flooding is likely to impact coastal development, infrastructure and fresh water resources most significantly. It is to be expected that these findings are rather the rule than the exception for low-lying coastal areas of tropical islands, especially since many Caribbean reefs are more severely degraded than those found along Bonaire. We, therefore, recommend that coral reef management efforts focusses on restoring natural coral barriers in regions that are most vulnerable to overflowing to prevent severe social and economic implications in the near future.
\end{abstract}




\section{Introduction}

In many ecosystems, bioengineered structural habitat complexity (e.g. trees, grasses or kelp) is a vital component promoting biodiversity by offering more potential niches and facilitating complex ecosystem functioning (MacArthur and MacArthur 1961; Estes and Palmisano 1974; Bruno and Bertness 2001). On tropical reefs such architectural heterogeneity is provided by the calcium carbonate skeleton of corals (Connell 1978; Luckhurst and Luckhurst 1978). $\mathrm{CaCO}_{3}$ precipitation by corals, in particular, underpins the biological success of coral reef systems with regard to functioning and facilitating an extraordinarily rich diversity (Graham and Nash 2013; Kennedy et al. 2013; Newman et al. 2015). Ancillary to this, reef structural complexity provides a natural barrier which increases frictional dissipation of wave energy.

The rigid skeleton of corals significantly increases both height and roughness of the bottom (Hearn 1999; Monismith et al. 2015). Ferrario and colleagues (2014) point out that a healthy reef reduces wave energy on average by $97 \%$ and wave height by $84 \%$. By diminishing the energy of waves at the shoreline these reefs have allowed human populations to settle and develop tropical coastal areas (Ferrario et al. 2014; Harris et al. 2018). Worldwide, and more specifically in the Caribbean, however, many reefs currently struggle to maintain a net positive carbonate budget (i.e. vertical accretion) (Eakin 1996; Perry et al. 2008; Eyre et al. 2018; Perry et al. 2018). Anthropogenic disturbances have resulted in reduced structural complexity and subsequent reef flattening (Alvarez-Filip et al. 2009). Over the past decades coral reef environments have seen fundamental ecological change due to various global and local stressors associated with human population expansion such as warming sea water, reduced water quality, loss of herbivores and disease outbreaks (Hughes 1994; Hughes et al. 2003; Jackson et al. 2001; Fabricius 2005). Consequently, coral cover has declined substantially (Bak et al. 2005; Gardner et al. 2003; De'ath et al. 2012; Jackson et al. 2014; De Bakker et al. 2017). Furthermore, on many reefs the coral community composition shifted from historically dominant frameworkbuilding species (Acropora spp. Orbicella spp. and Porites spp.) (Pandolfi and Jackson 2006) towards assemblages dominated by small opportunistic species that are incapable of facilitating the same structural complexity or support such high biodiversity (Edinger and Risk 2000; Mumby et al. 2008; Alvarez-Filip et al. 2011a; Perry et al. 2015a; De Bakker et al. 2016b). The unprecedented decline in abundance and cover of calcifying organisms is paralleled by an increase in abundance and activity of biological eroders (in particular endolithic macroborers and microborers), which appear to thrive in warmer, more eutrophic waters (Perry et al. 2014; Glynn and Manzello 2015; Schönberg et al. 2017b; Achlatis et al. 2017; Webb et al. 2017; Silbiger et al. 2018). Moreover, they benefit from the increased availability of substratum following coral mortality (Schönberg et al. 2017a).

Aforementioned change in reef configurations has negatively affected the carbonate balance on many coral reefs, often tipping the scale towards net erosion. The consequential structural degradation severely reduces the hydraulic roughness of the shallow bottom (Ferrario et al. 2014; Harris et al. 2018). Dissipation of wave energy by well-developed reefs correlates linearly to intensified water movement, even at extreme events such as storms or hurricanes (Ferrario et 
al. 2014). Healthy reefs thus play a crucial role in risk reduction during such extreme events (Blanchon et al. 2010). Although structural remnants of deceased corals and the general geomorphology of the reef will, to some extent, still aid in reducing wave energy, the dissipation will become increasingly less efficient when the carbonate skeleton of corals is further degraded. A major concern is that the impact of waves on the shoreline will only be intensified through rising sea levels. Living corals have the capacity to track rising sea levels by consistently minimizing the thickness of the water column overlying the reef through vertical growth. Since water depth and shoaling play an important role in amplifying wave energy, actively growing living reefs serve as highly efficient wave breakers preventing inundation and coastal erosion. Degraded reefs with little three-dimensional complexity lack the capacity to substantially reduce wave energy, which has direct negative implications for coastal areas (Sheppard et al. 2005; Harris et al. 2018). Increased frequency of flooding, for instance, severely impacts coastal developments and infrastructure and salinifies freshwater lenses (Terry and Falkland 2010; Storlazzi et al. 2018). Low-lying tropical shores, in particular, are expected to experience more frequent flooding when reef topography is declining further and sea-level continues to rise (Hopkinson et al. 2008; Slangen et al. 2014; Quataert et al. 2015; Vitousek et al. 2017; Storlazzi et al. 2018).

It seems apparent that the ability to prevent inundation and the capacity of coral reefs to track sea-level rise varies strongly depending on local conditions, including the existing coral community, hydrodynamics, coastal morphology and adjacent human impact (Perry et al. 2013; Perry et al. 2015b; Januchowski-Hartley et al. 2017; Perry et al. 2018). Studies examining reef accretion potential at fine spatial resolution and linking observed variation to local conditions are, however, scarce. Here, we used extensive field data collected from the leeward coast of Bonaire (southern Caribbean) to define the current growth potential of shallow reefs. We attempt to predict the capacity of these reefs to protect the coastline in view of projected sealevel rise scenarios. Bonaire's reefs are considered to be amongst the healthiest reefs in the Caribbean relative to historic baselines (Jackson et al. 2014), but do they still have the growth capacity to track sea level rise? It is likely that coastal regions with severely degraded reefs in front will become increasingly more susceptible to wave induced impact (Alvarez-Filip et al. 2011c). This would be particularly evident during extreme weather events (e.g. hurricanes) or when exposed to extreme swell. In contrast, regions that are located more distant from urban areas are expected to have maintained considerable net vertical accretion potential. The variation in coastal exposition and human population density makes Bonaire the ideal case study to investigate the ramifications of spatial variation in reef carbonate budgets and growth potential in relation to predicted sea-level change. In this study, maximum reef accretion potential and local morphology are assessed at fine spatial resolution to identify high-risk areas on which conservation and reef restoration efforts should be focussed. 


\section{Materials and Methods}

\section{Study site and data collection}

Dedicated surveys to quantify net coral reef calcification were carried out on the shallow reefs of the southern Caribbean island of Bonaire $\left(12^{\circ} 9^{\prime} \mathrm{N}, 68^{\circ} 16^{\prime} \mathrm{W}\right)$ (Figure 5.1). Survey efforts were confined to the leeward side of the island and conducted between September and December 2017. Substantial reef formations are concentrated on this side of the island and here human activity is highest as well. The general reef profile on the leeward side comprises a lowerterrace zone (LT, $\sim 3$ to $7 \mathrm{~m}$ depth) that gradually slopes from the shore to the upper drop-off zone (DO) at approximately 10 to $15 \mathrm{~m}$ depth, after which the reef steeply runs down (usual angle between $20^{\circ}$ and $50^{\circ}$ ) to a depth of 25 to $50 \mathrm{~m}$ towards a second terrace (Bak 1977; Van Duyl 1985). Net coral reef calcification data (in $\mathrm{G}$, where $\mathrm{G}=\mathrm{kg} \mathrm{CaCO}_{3} \mathrm{~m}^{-2} \mathrm{yr}^{-1}$ ) was collected from 115 sites (including Klein Bonaire), separated by a predefined distance of $\sim 500 \mathrm{~m}$ and covering the majority of the leeward reef stretch (Figure 5.2). Per site and in each zone (LT and DO), replicate transect lines $(25 \mathrm{~m}$ ) were positioned in series (starting points $10 \mathrm{~m}$ apart) and parallel to the orientation of the reef front (Hill and Wilkinson 2004).

\section{Assessment of net carbonate calcification}

Data to determine gross and net carbonate production and erosion were collected following procedures as outlined by Perry et al. (2012) in the ReefBudget methodology. Some minor adaptations were implemented, which we expand on below. In the ReefBudget approach net coral reef calcification is estimated as the cumulative $\mathrm{CaCO}_{3}$ produced by coral and calcifying algae minus the chemical dissolution and mechanical removal of $\mathrm{CaCO}_{3}$ through bioeroding activities. Bioeroding organisms include parrotfish, sea urchins, boring macrofauna and microborers. The percentage cover of all biotic and abiotic groups was measured to the nearest $\mathrm{cm}$ directly below the transect line and following the full contour of the reef. To minimize spatial correlation the two benthic transects per depth at each site were positioned at the end of the two main transect lines (from 15 - $25 \mathrm{~m}$ ), separating them by a distance of $40 \mathrm{~m}$. Scleractinian corals were visually identified to species level. The difference between the contour-following length and the horizontal main transect line $(10 \mathrm{~m})$ was used to approximate reef rugosity (Risk 1972). As a second complexity measure the maximum reef height, measured from the bottom, was determined every $2 \mathrm{~m}$ (within $2 \mathrm{~m}$ of the transect line), yielding six values for reef elevation for each main $10 \mathrm{~m}$ benthic transect.

Coral $\mathrm{CaCO}_{3}$ production was calculated based on the measured cover for each species and species specific growth rates and skeletal density. Because growth rates and skeletal density vary with depth, different constants were applied for the two reef zones (ReefBudget, Perry et al. 2012 and references therein). The sum of the production rates for each encountered colony of a certain species resulted in a gross coral carbonate production $\left(\mathrm{kg} \mathrm{m}^{-2} \mathrm{yr}^{-1}\right)$ per transect. Carbonate production by crustose coralline algae (CCA) was calculated using cover and a standard calcification rate derived from published Caribbean data (Perry et al. 2012 and references therein). Total carbonate removed by parrotfish was estimated based on the 


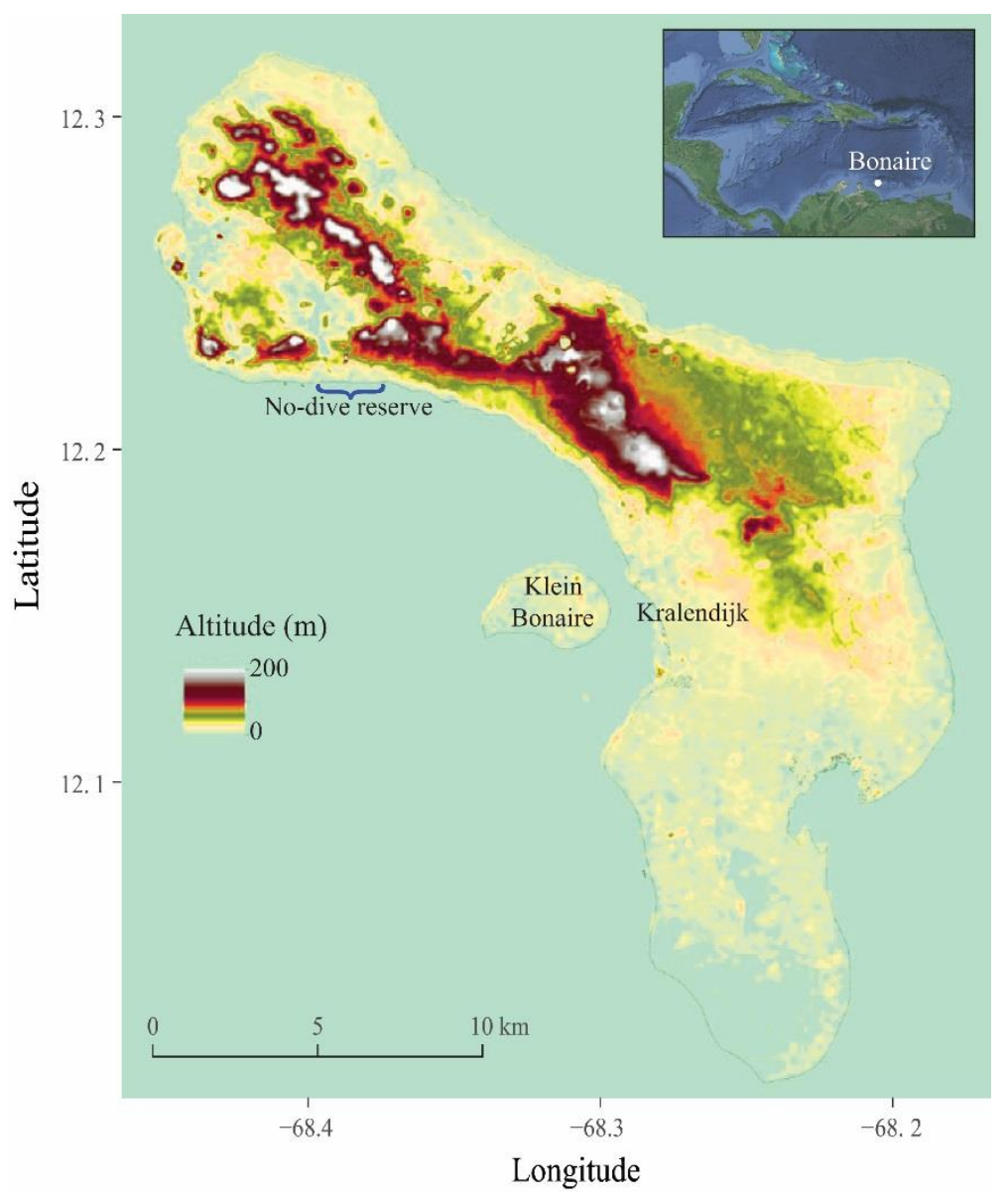

Figure 5.1. Altitude map of Bonaire. Light shades indicate the vulnerable low-lying coastal areas in the south of the island. The location of the town of Kralendijk, Klein Bonaire and the King Willem-Alexander no-dive reserve are indicated. Inset shows the position of Bonaire in the southern Caribbean Sea. Figure is adapted from Mücher et al. (2017). (AsterDEM Source: NASA and Google earth V 7.3.1.4507).

erosion potential of each individual fish encountered within a $5 \mathrm{~m}$ wide belt following the main $25 \mathrm{~m}$ transect lines. Individual erosion was calculated using fish biomass (derived from fork length), life stage (juvenile, initial, terminal) bite rate, ratio of bites leaving scars and mass eroded per bite. The sum of erosion by all observed fish resulted in an estimate of gross carbonate removal by parrotfish. Parrotfish erosion was set to zero when no hard substratum was available within the transect (i.e. only sand). The contribution of sponges to gross bioerosion was determined using species specific erosion rates for chemical and mechanical erosion per unit of infested substrate as defined by De Bakker et al. (2018). Cover for all excavating sponges was measured within $50 \mathrm{~cm}$ on both sides of the transect line $\left(10 \mathrm{~m}^{2}\right.$ in 
total). Sea urchin erosion was quantified in a belt transect as well, but at $1 \mathrm{~m}$ on each side of the main transect line (20 $\mathrm{m}^{2}$ in total). Size and species of each urchin encountered within the belt transect was related to a constant for calcium carbonate removal derived from the ReefBudget procedure. The impact of microborers was determined conform the ReefBudget procedure (Perry et al. 2012), where an erosion constant is given for erodible substrate (excluding sand and substratum covered by larger sessile organisms such as corals or sponges) at $5 \mathrm{~m}$ and $10 \mathrm{~m}$ depth. All constants and procedures of the ReefBudget methodology are described in detail on http://geography.exeter.ac.uk/reefbudget/. Final estimates for net coral reef calcification for each transect were converted to estimates of potential annual reef accretion. Maximum reef accretion potential (RAP in mm year ${ }^{-1}$ ) was determined conform (Perry et al. 2015b and Perry et al. 2018), by applying corrections for sediment infill (25\% of the sediment produced by parrotfish and $50 \%$ of the sediment produced by other bioeroding organisms), framework porosity (30\% for head and massive coral dominated assemblages, $70 \%$ for branched and tabular dominated assemblages, and 50\% for mixed coral assemblages) and natural framework erosion through physical processes (20\% for sheltered sites and $50 \%$ for exposed sites). RAP was subsequently linked to various projections of sea level rise (2018 IPCC report scenarios RCP2.6, 4.5 and 8.5). A basic risk assessment based on the RAP and local environmental conditions was conducted, identifying coastal areas most prone to inundation (Table 5.1). Threshold values were assigned a value of 1 to 4 for low to critical, final scores per site were the sum of these values for all five parameters. Data on the environmental parameters terrace width and wind exposure were acquired from Van Duyl (1985) and the FORCE project (http://webgis.forceproject.eu/forcewebgis.html\#), respectively. Estimates for coastal height were derived from digital surface model (DSM, ALOS World 3D) captured by the Japan Aerospace Exploration Agency's (JAXA).

Table 5.1. Parameters and threshold values included in the risk assessment for coastal flooding. Low to critical indicates the level of risk for each individual parameter. RAP: maximum reef accretion potential.

\begin{tabular}{|l|c|c|c|c|} 
& Low & Moderate & High & Critical \\
\hline Vertical reef elevation $(\mathrm{cm})$ & $>100$ & $50-100$ & $20-50$ & $<20$ \\
\hline Coastal height $(\mathrm{m})$ & $>10$ & $5-10$ & $2-5$ & $<2$ \\
\hline RAP $\left(\mathrm{mm} \mathrm{year}^{-1}\right)$ & $>9.6$ & $6.9-9.6$ & $4.9-6.9$ & $<4.9$ \\
\hline Terrace width $(\mathrm{m})$ & $>175$ & $100-175$ & $50-100$ & $<50$ \\
\hline Wind exposure $\left(\mathrm{J} \mathrm{m}^{-3}\right)$ & $<350$ & $350-550$ & $550-750$ & $>750$ \\
\hline
\end{tabular}




\section{Site clustering with contiguity constraint}

Multivariate regression tree analysis (MRT) (De'ath 2002; De'ath 2006) was used to identify distinct spatial clusters across the leeward coast of Bonaire in both the LT and DO zone. Cluster analysis is commonly used to define types of assemblages (Anderberg 1973; Legendre and Legendre 2012) and MRT is a natural constrained extension of univariate regression trees with a multivariate response instead of a univariate response variable. While MRTs are generally used for community data, we applied MRT analysis to mean values per site for the different constituents of the carbonate budget (coral production; CCA production; parrotfish erosion; macroborers; urchin erosion and microborers) with latitude and longitude as constraining explanatory contiguity variables (Borcard et al. 2011). In the analysis all constituents were standardized to the mean and standard deviation (Faith et al. 1987). Tree size (i.e. the number of leafs) was selected based on standard error cross-validation (Appendix 5.A) (De'ath 2002). The acquired clustering could subsequently be related to environmental variation. Individual descriptive reef characteristics related to reef accretion and coastal protection potential (rugosity, mean height, substrate and live coral cover) for the acquired clusters (levels) were modelled by means of linear regression and analysis of covariance. All descriptive data were fourth-root transformed prior to analyses. Generalized additive modelling (GAM) was applied to predict the minimum coral cover needed to support a net reef accretion able to cope with sealevel rise. For the GAM, with a Gaussian distribution, RAP values were log-transformed and a fourth-root transformation was applied to live coral cover. A model including both zones separately explained less deviance $(74.3 \%$ compared to $76.2 \%$ for the model combining both zones) and individually fitted responses were virtually identical $(F=5.52, p=0.72)$. Exploration of the model residuals with variogram plots indicated there was no need to account for spatial auto-correlation. Data processing, MRT analyses (De'ath 2006) and statistical tests were conducted in the $\mathrm{R}$ programming environment v3.4.1 ( $\mathrm{R}$ Core Team 2013) using the following packages: mgcv (Wood 2012), Mvpart (De'ath 2006) and stats (R Core Team 2002). Since the aim of this study was to determine the reef accretion potential for the entire leeward reef or larger stretches of reef and not for individual sites, four transects were assessed per site (two in each zone) instead of six as proposed in the ReefBudget methodology. As such, the statistical power of the analyses lies not in the number of transects per site, but in the total number of surveyed reef sites.

\section{Results}

Mean net carbonate production along the leeward coast of Bonaire was $1.99 \mathrm{G}(95 \% \mathrm{CI}: 1.41$ to 2.60) in the drop-off (DO) zone and $0.22 \mathrm{G} \mathrm{(95 \%} \mathrm{CI:}-0.24$ to 0.72$)$ on the lower terrace (LT). Considerable variation was observed across sites with $\mathrm{G}$ ranging from -5.72 to 21.12 (DO) and -5.98 to $26.48(\mathrm{LT})$, on a transect level. Almost 50\% $(\mathrm{n}=113)$ of all transects in the LT zone had a negative net production (Figure 5.2A). In the DO this was 32\% $(\mathrm{n}=73$ ) (Figure 5.2C). Eight LT transects had $100 \%$ cover of sand. In contrast, 23 DO and 12 LT transects had a net production higher than $10 \mathrm{G}$. In four of these LT transects A. cervicornis colonies accounted for the majority of the carbonate production. In all other sites with a production greater than $5 \mathrm{~kg}$ $\mathrm{m}^{-2}$ year $^{-1}$ the coral community was dominated by Orbicella spp. 
Multivariate regression tree (MRT) analysis with the six individual carbonate budget constituents as multivariate response variables resulted in a four-leafed tree for the LT as the arguably best tree and a five-leafed tree for the DO zone. In both trees the splits were constrained by the position variable latitude, and for DO tree also by longitude (Appendix 5.A). The fourleafed tree for the LT did not necessarily represent the cross-validation with the smallest estimated predicted error (Appendix 5.A), but was still within 1 SE of the best tree (see De'ath 2002) and did subjectively provide the most logical spatial clustering. Overall, splits of the LT and DO tree were spatially highly comparable between zones. This clustering, based on gross carbonate production and erosion variables resulted in the ordination of clusters along the coast of Bonaire as displayed in Figure 5.2A and Figure 5.2C.

Evaluation of the individual budget constituents reveals that CCA contributes marginally (on average $<1 \%$ ) to the gross carbonate production on the leeward reefs of Bonaire (Figure 5.2B \& Figure 5.2D). While $\mathrm{CaCO}_{3}$ production by CCA appears to be higher on less degraded reefs (sites farthest away from the capital), it is unsurprising that overall the vast majority is produced by corals. Parrotfish were the main eroders of $\mathrm{CaCO}_{3}$ on these reefs. The contribution of excavating sponges and micro-bioerosion can be considerable, but largely depends on the availability of substratum. Erosion by sea urchins was negligible along on the entire shallow reef as densities were extremely low (on average 0.08 urchins $\mathrm{m}^{-2}$ ).

Figure 5.2. Multivariate regression tree clustering and carbonate budget constituents. Location of the surveyed sites $(n=115)$ along the leeward coast of Bonaire and Klein Bonaire. Symbols indicate distinct clusters defined through multivariate regression tree analysis with a spatial contiguity constraint on the lower-terrace $(\boldsymbol{A})$ and drop-off zone $(\boldsymbol{C})$. Colours represent ranges of mean net carbonate production (in $G$, where $G=\mathrm{kg} \mathrm{m}^{-2}$ year $\mathrm{r}^{-1}$ ). Line inserts on the $x$ and y-axis represent breaks (tree nodes) that are characterized by a threshold of latitude or longitude, defining the individual clusters. Cluster numbers are given next to each clusters. (B) and $(\boldsymbol{D})$ : mean contribution of the individual carbonate budget constituents to gross production $\left(\mathrm{kg} \mathrm{CaCO}_{3} \mathrm{~m}^{-2}\right.$ year $\left.{ }^{-1}\right)$ and erosion $\left(\mathrm{kg} \mathrm{CaCO}_{3} \mathrm{~m}^{-2}\right.$ year $\left.{ }^{-1}\right)$ for each cluster. Error bars indicate $95 \%$ confidence limits. 

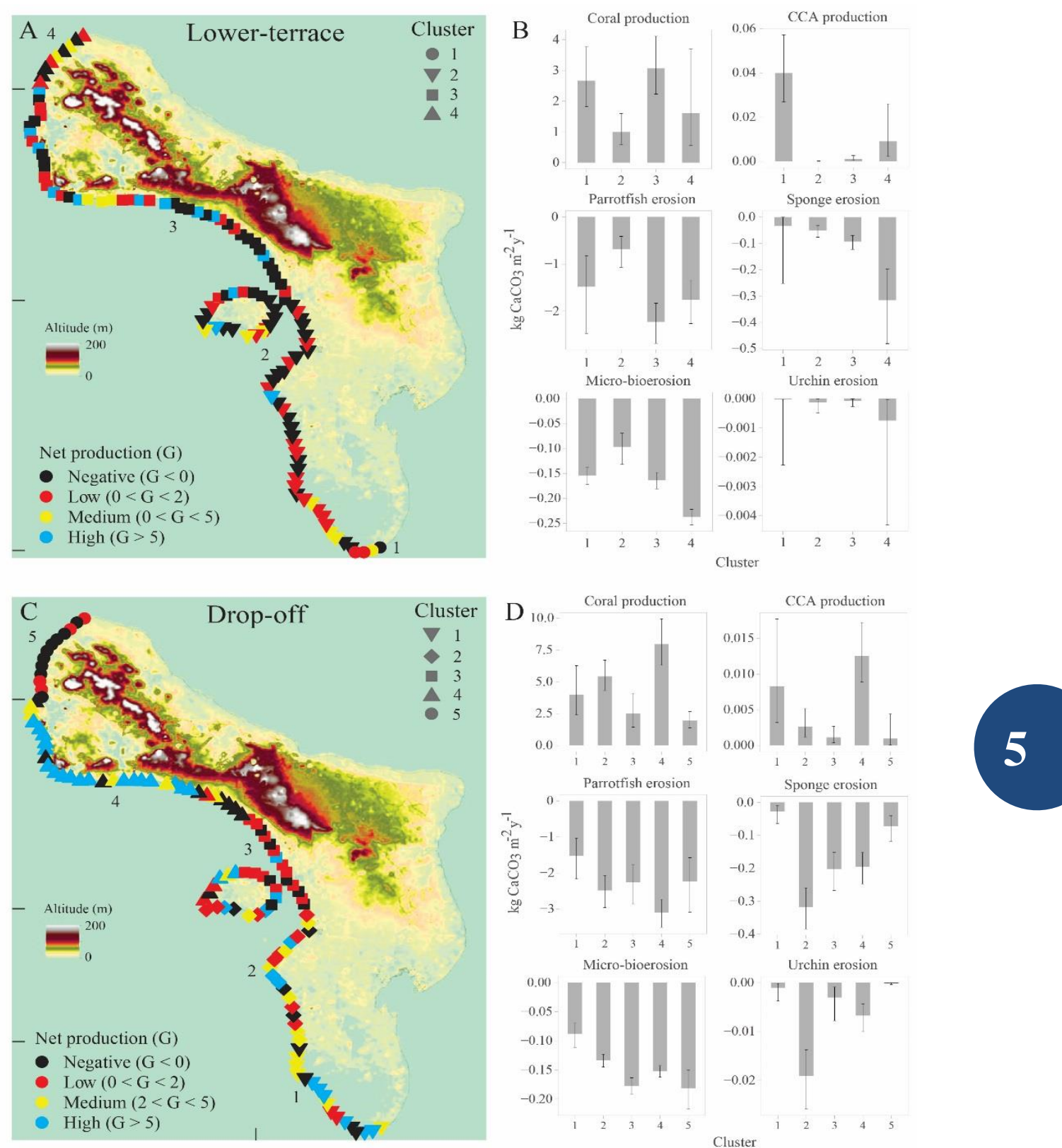

Comparing general reef characteristics across clusters and zones revealed considerable variation along the coast (Figure 5.3 \& Appendix 5.B). Within the DO zone (bottom group of graphs), reefs in cluster 4 are markedly well developed compared to the other four clusters. Reefs within this cluster generally still have high coral cover and complex architectural structure (Figure 5.3 \& Appendix 5.B). The same, albeit to a lesser extent, is true on the LT for the sites in cluster 1 (south point) and cluster 3 which shares a considerable number of sites with DO cluster 4 (Figure 5.2). These relatively well developed reefs encountered north of Kralendijk and at the 
south point of the island also generally have low sand cover (Appendix 5.B). Highest net carbonate production was found within the King Willem Alexander no-dive reserve (Figure 5.1 \& Figure 5.2). Here mean net production was $10.94 \mathrm{G}$ (95\% CI: 7.22 - 15.94) and $13.56 \mathrm{G}$ (95\% CI: 10.38 - 17.41) in the lower-terrace and drop-off zone, respectively. This could largely be attributed to a still considerable mean coral cover of $25 \%$ (LT) and $30 \%$ (DO). The coral community in the no-dive reserve was also dominated by Orbicella spp. colonies that have created extraordinarily rugose reefs in both zones. Rugosity within the reserve resulted in a surface enlargement of $32 \%$ to $66 \%$ (LT) and $95 \%$ to $133 \%$ (DO), with a mean reef elevation from the bottom of $24.4 \mathrm{~cm}$ and $104.1 \mathrm{~cm}$, respectively. Other clusters were generally characterized by the absence of well-developed structural complexity, generally low live coral cover and, in particular on the southern LT cluster 2, little hard substratum and substantial sandy areas. In these clusters, the majority of sites are currently already in a state of erosion or maintain a net growth rate of less than $2 \mathrm{G}$ (Figure 5.2). This is also evidently the case for the reef in front of Kralendijk where almost all LT sites are net eroding. Reefs in the northern most clusters (LT cluster 4 and DO cluster 5) were defined by low-complexity reefs with marginal net production, however, in contrast to the southern clusters these exposed reefs have fewer sandy patches.
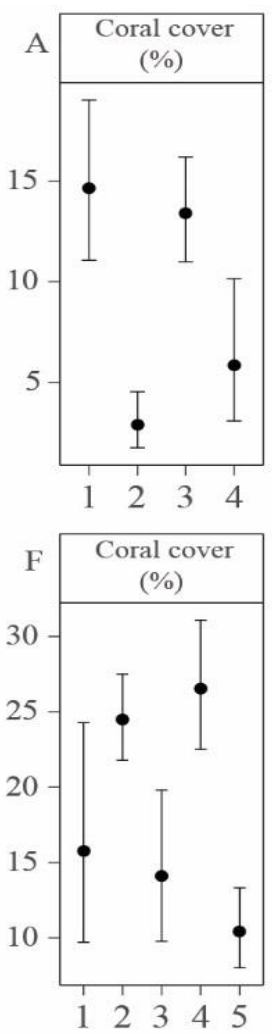
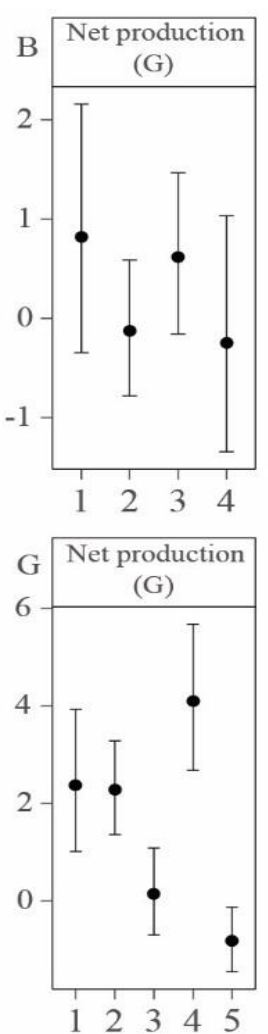
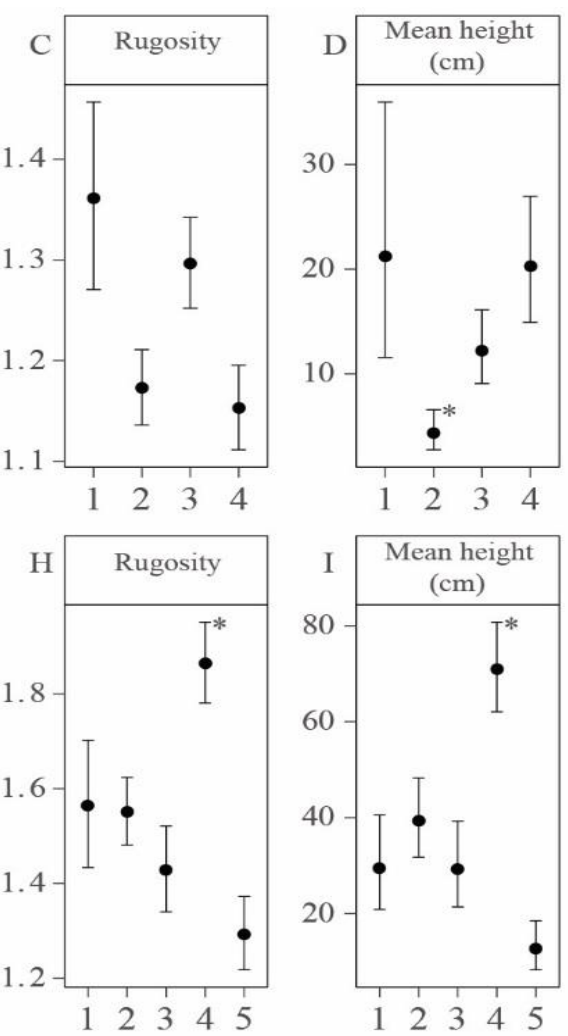
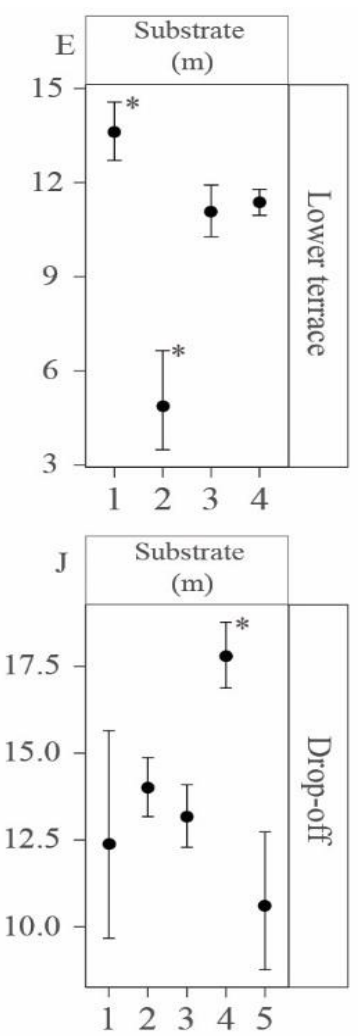

Cluster 
Figure 5.3. Cluster characteristics. Main descriptive reef characteristics related to reef accretion and coastal protection potential for each cluster. Points represent the mean of all transects within each cluster, segments define $95 \%$ confidence limits. $(\boldsymbol{A}, \boldsymbol{F})$ Percentage of the total 3-dimensional transect length covered by live coral (including Millepora spp.). (B,G) Net carbonate production (gross production minus gross erosion), given in $\mathrm{G}$ ( $\mathrm{kg} \mathrm{CaCO} \mathrm{Cm}^{-2}$ year$\left.{ }^{1}\right)$. (C,H) Rugosity based on the chain and tape technique. (D,I) Mean reef height from the bottom (in $\mathrm{cm}$ ) collected every $2 \mathrm{~m}$ along each transect line. $(\boldsymbol{E}, \boldsymbol{J})$ total length (including $3 D$ surface enlargement) of the transect taken up by hard substratum or living benthic biota (i.e. everything except sand). Stars indicate clusters that are significantly different $(p<0.05)$ from all other clusters.

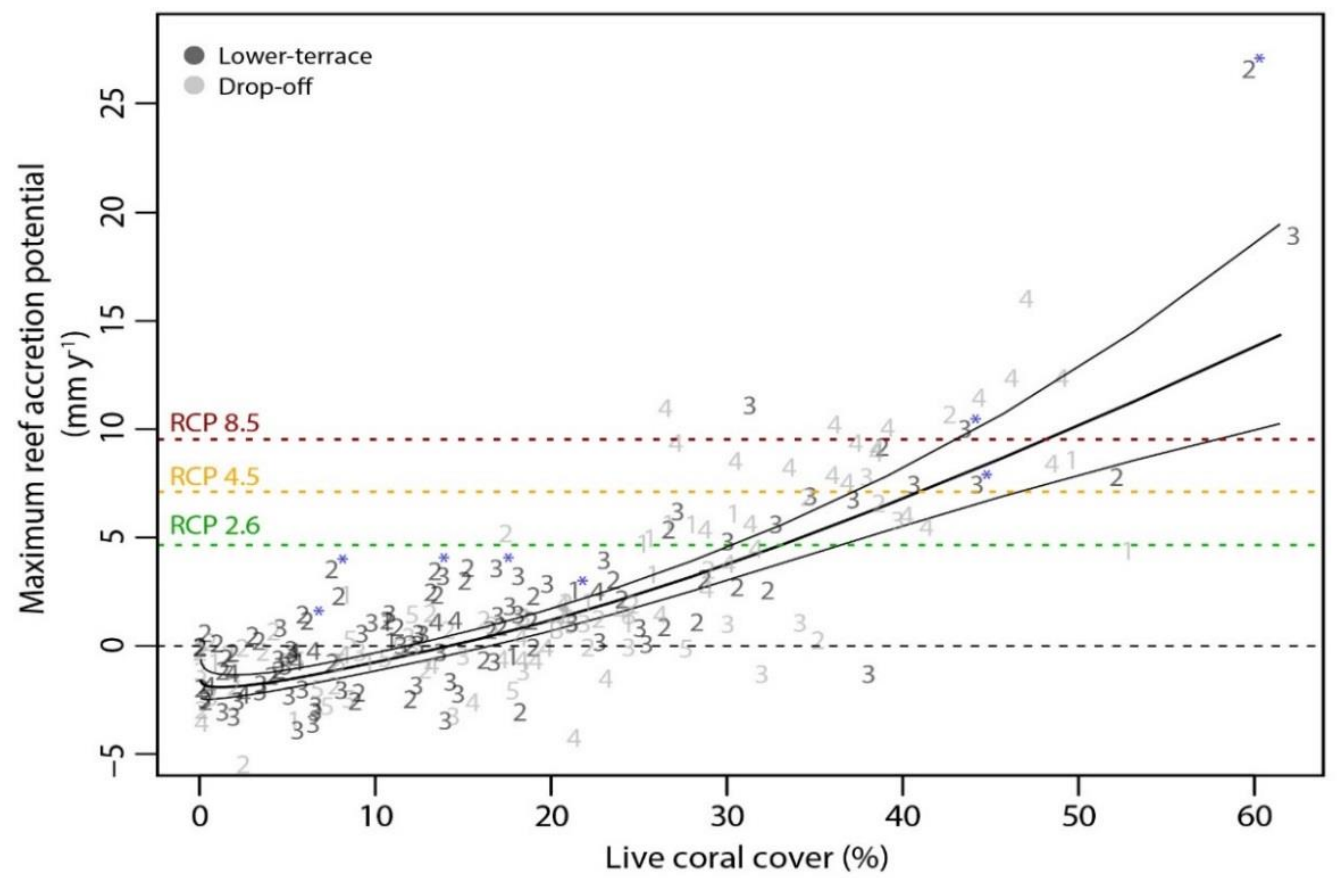

Figure 5.4. Relation between net maximum reef accretion potential and the percentage of live coral cover on the shallow reef of Bonaire. The solid black curve represents the fitted response (RAP) versus live coral cover, with the mean coral cover and RAP at each site plotted in dark grey (lower-terrace zone) and light grey (drop-off zone). Numbers refer to the cluster in which the site was located. The predicted 95\% confidence interval limits are shown by the upper and lower solid black curves. The horizontal black dashed line represents a RAP of zero. The point where this line intercepts the fitted curve can tentatively be regarded as the approximate minimum required coral cover percentage to maintain the current structural complexity of the Bonaire reefflat. Coloured dashed lines indicate the levels of carbonate production necessary to keep up with ICPP projected sea-level rise scenarios for Bonaire: RCP 2.6 (green, $4.9 \mathrm{~mm}$ SLR year ${ }^{-1}$ ), RCP 4.5 (yellow, $6.9 \mathrm{~mm} \mathrm{SLR} \mathrm{year}^{-1}$ ) and RCP 8.5 (red, $9.4 \mathrm{~mm} \mathrm{SLR} \mathrm{year}^{-1}$ ) (Flato et al. 2013; Slangen et al. 2014). Blue stars indicate sites containing Acropora cervicornis stands. 
The estimated mean maximum reef accretion potential (RAP) of the shallow reef was $1.33 \mathrm{~mm}$ year $^{-1}$ (95\% CI: 1.09-1.58). RAP for the LT zone was $0.67 \mathrm{~mm}_{\text {year }}{ }^{-1}$ (95\% CI: $0.38-0.97$ ) and $2.05 \mathrm{~mm}$ year $^{-1}$ (95\% CI: 1.67-2.45) for the DO zone. Across the studied reef, highest RAP was found in DO cluster 4, where mean accretion was $3.73 \mathrm{~mm} \mathrm{year}^{-1}$ (95\% CI: 2.85-4.70) (Appendix 5.B). On the lower terrace - the zone most prominently involved in reducing wave energy - only 14 sites have maintained a reef accretion potential capable of tracking the most optimistic projections for sea-level rise (RCP2.6: $4.9 \mathrm{~mm} \mathrm{SLR}_{\text {year }}{ }^{-1}$ ), eight sites can currently track the RCP 4.5 (6.9 $\mathrm{mm} \mathrm{SLR} \mathrm{year}^{-1}$ ) and only four sites the RCP 8.5 (9.4 mm SLR year $\left.{ }^{-1}\right)$. For the DO zone this goes for 23, 12 and 1 sites, respectively. RAP for the King Willem Alexander no-dive reserve was $2.58 \mathrm{~mm} \mathrm{year}^{-1}$ (95\% CI: 2.31-2.85) at the DO and $2.33 \mathrm{~mm}$ year $^{-1}$ (95\% CI: $\left.1.97-2.71\right)$ at the LT.

The threshold for live coral cover at which RAP on the shallow leeward reef of Bonaire (LT and DO) becomes negative lies at approximately 14.4\% (Figure 5.4). Below this level, thickness of the water column overlying the reef will start to increase with time even without taking into account SLR. The model also suggest that $34 \%$ coral cover would be needed to track the most conservative sea-level rise prediction (RCP 2.6). Coral cover would have to be roughly $40 \%$ (RCP 4.5) or $48 \%$ (RCP 8.5) to follow more pessimistic, but likely more realistic, SLR projections (Figure 5.4). The vast majority of the transects with a RAP above the RCP 2.6 level are found in LT cluster $3(n=11)$, DO cluster $4(n=20)$, at the south point and around Klein Bonaire. Obviously this depends also on the type of coral that dominates the community. Several of the few southern LT sites that ranged above RCP 2.6 managed such RAP largely due to the presence of A. cervicornis stands (Figure 5.5). All LT with A. cervicornis cover had a positive RAP. While currently LT reefs are predominantly dominated by sand or turf covered substratum (Appendix 5.B), with some small opportunistic coral colonies, they were formerly characterized by extensive fields of highly productive A. cervicornis (Figure 5.5).

\section{Discussion}

Our findings show that the majority of the shallow reef of Bonaire is hardly growing and in many places most likely eroding away. This historically low gross calcium carbonate production can by large be attributed to the substantial loss in coral cover over the past decades (Bak et al. 2005; Sommer et al. 2011; De Bakker et al. 2017). Additionally, a shift in coral assemblages to species that produce less carbonate per unit area covered (Alvarez-Filip et al. 2013; De Bakker et al. 2016b) and an increase in benthic bioerosion (Glynn and Manzello 2015) severely reduced net calcium carbonate deposition. The region-wide mass mortality of Acropora spp. as a result of white band disease (Bak and Criens 1981; Gladfelter 1982), in particular, meant a considerable decline in the carbonate production on shallow coral reefs (Aronson and Precht 2001). Bonaire's coral reefs are generally considered to have experienced less ecological decline in relation to other Caribbean reefs (Kramer 2003; Steneck et al. 2013; Jackson et al. 2014), yet they have not been free of stress (De Bakker et al. 2016b; De Bakker et al. 2017). The results presented in the current study also indicate that minimized reef degredation only applies to a small fraction of the leeward reef. Indeed, coral cover was still remarkably high in some regions 
(Figure 5.3 \& Appendix 5.C), but mean cover on the entire shallow reef was only a mere $12 \%$. This is in range with, but slightly below, the Caribbean average (Jackson et al. 2014). Concurrently, there was considerable spatial variation in net carbonate production across the leeward coast, with some near-pristine sites approaching pre-industrial accretion rates (Vecsei 2001) while other regions, most notably in front of Kralendijk, have very little coral cover and experience substantial reef erosion. The observed variation in carbonate production and maximum reef accretion potential across the 460 transects and in comparison to previous calcification studies on Bonaire is remarkable. This variation at high spatial resolution reveals that transect placement or the inclusion of too few sites can strongly affect the outcome of survey studies. Caution should therefore be exercised when drawing island-scale conclusions in case latter issues apply. Insufficient sampling can easily lead to wrong elucidation on the state of a reef, which in turn can result in inadequate management efforts.

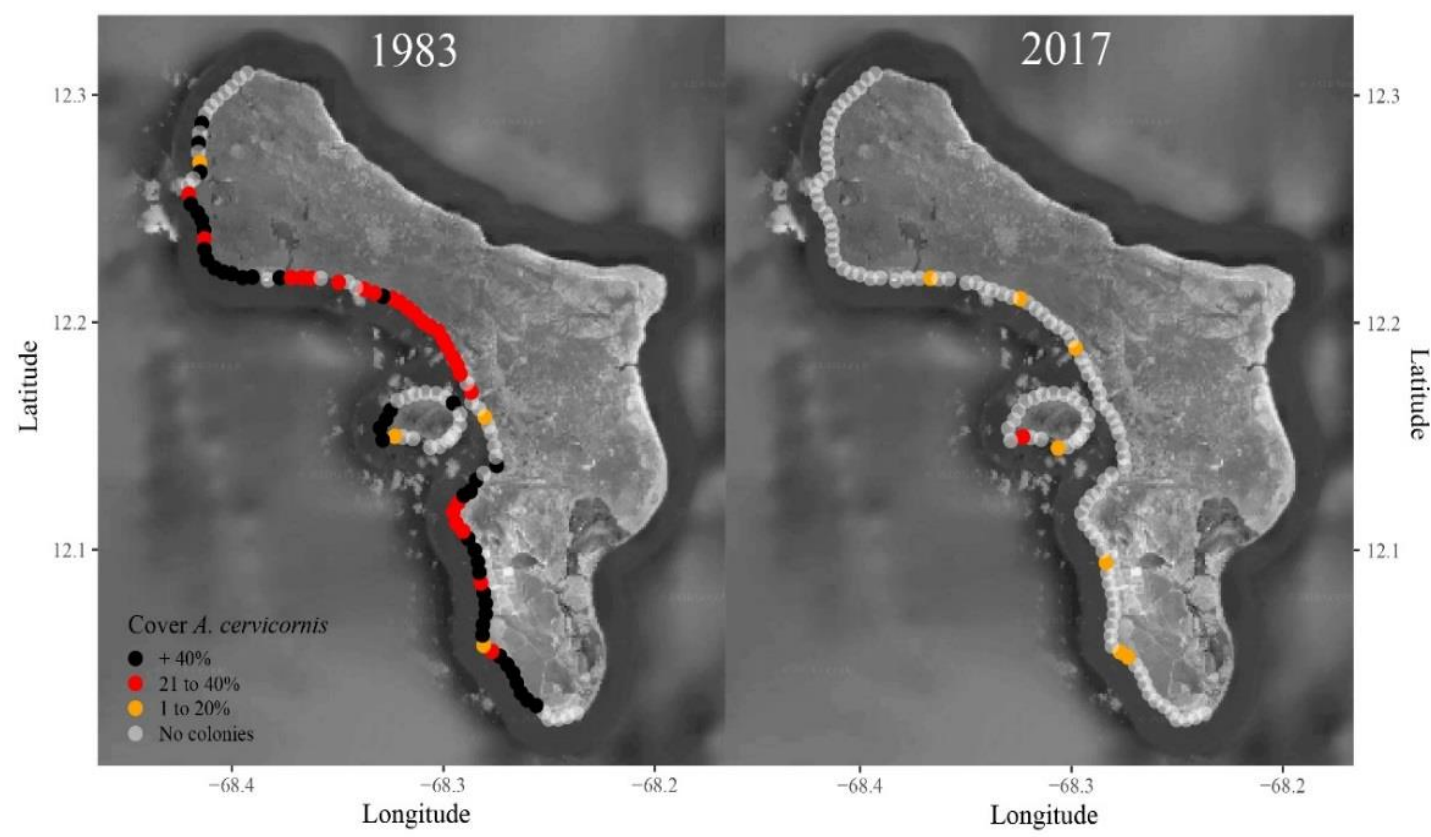

Figure 5.5. Decline in Acropora cervicornis since the 1980s. Estimated percentage cover of A. cervicornis along the leeward coast of Bonaire and Klein Bonaire based on data collected between 1981 and 1983 (Van Duyl 1985) and in 2017 (this study). Dots show the 115 sites as surveyed in 2017 (this study), colours represent ranges in percentage cover of the total benthic community.

Overall, the average estimate of vertical reef accretion on the shallow reef of Bonaire was approximately $2 \mathrm{~mm}$ year ${ }^{-1}$, which is substantially lower than even the most optimistic projections for average of mean sea-level rise ( 4.9 $\mathrm{mm} \mathrm{year}^{-1}$, source: ICPP) (Flato et al. 2013). Although this study focusses only on the shallow reef, we predict similar limited reef accretion potential across the reef slope since the reefs of Bonaire have experienced substantial 
degradation, at least down to $40 \mathrm{~m}$ depth, in recent decades (De Bakker et al. 2017). The latter implies that the majority of Bonaire reefs will not be able to keep up with sea-level rise which raises concern on the social and economic implications this may have for the island (i.e. caused by frequent inundation of coastal areas) (Ferrario et al. 2014; Quataert et al. 2015; Storlazzi et al. 2018).

The average estimated net $\mathrm{CaCO}_{3}$ production by the shallow reef habitat of Bonaire is $1.1 \mathrm{~kg}$ $\mathrm{m}^{2}$ year ${ }^{-1}$. This is below previous recordings on Caribbean reefs (Stearn et al. 1977; Scoffin et al. 1980), but comparable to some more recent records around Florida and de Greater Antilles (Perry et al. 2018). Perry and colleagues (Perry et al. 2012; Perry et al. 2013) found the average production for four Bonaire reef sites to range from -1.74 to $15.25 \mathrm{G}$ on the LT and 3.51 to 16.68 $\mathrm{G}$ in the DO zone. This is comparable to our findings, although evidently the inclusion of 115 sites for each zone results in a better representation of intra-island variability. Nevertheless, their site specific mean net production rates (at the no-dive reserve $5 \mathrm{~m}$ and $10 \mathrm{~m}$ and in front of Kralendijk - Calabas - $10 \mathrm{~m}$ ) were comparable to our findings at the nearest surveyed sites (Appendix 5.C: sites 87 and 28, respectively). We, however, found the lowest accretion rates on the southern reefs of Bonaire and around the densely populated capital (Kralendijk), a region which was hardly represented in aforementioned studies. Yet, these reefs are of great economic importance attributable to their potential to serve as a natural barrier protecting the extremely low-lying southern coastal area (including Kralendijk) from wave-induced erosion and frequent inundation. Several recent studies point out the augmented risk of annual flooding following climatological change (Vitousek et al. 2017; Harris et al. 2018; Storlazzi et al. 2018). They predict that under the current conditions of reduced reef accretion in combination with sea-level rise and changing storm patterns many low-lying tropical areas will already become uninhabitable in the coming decades (Hopkinson et al. 2008; Slangen et al. 2014; Quataert et al. 2015; Vitousek et al. 2017; Storlazzi et al. 2018). The shore of southern Bonaire only raises approximately $1 \mathrm{~m}$ or less above the mean sea level (Van Duyl 1985; Mücher et al. 2017) and is therefore markedly susceptible to inundation and erosion. Alarmingly, here the reefs that are supposed to diminish wave energy are currently in a particularly poor state with a negligible reef accretion $\left(<1 \mathrm{~mm} \mathrm{year}^{-1}\right)$ and still further disappearing structural 3D-complexity. Bonaire has indeed already been experiencing repeated flooding in this region during the passing of storms and hurricanes (https://www.youtube.com/watch?v=YvZuAhJ-7pg).

The applied risk assessment to define regions that are more vulnerable to inundation underlines the concern for coastlines in the southern and middle region of Bonaire (Figure 5.6). The majority of the coastal area in this region, including Kralendijk, is highly susceptible to inundation in the case of extreme weather events such as tropical storms and hurricanes or large swell. Most notably, this region is characterized by marginal elevation of the coastline and reefs with high coral cover, capable of maintaining considerable net reef accretion, is virtually absent. Similarly, the analysis points out that a well-developed reef, in combination with favourable local condition, indeed reduces the threat of flooding. Sites to which these conditions apply were found in the northern part of the island, around Klein Bonaire and at the southernmost tip of the island (Figure 5.6). Nonetheless, the majority of the leeward coastline is currently at risk of 
flooding despite the claim that the reefs of Bonaire rank among the best throughout the Caribbean. It should be pointed out that the present study does not specifically quantify the change in coastal protection service due to a reduction in accretion potential of coral reefs in the future. We aim to draw parallels to this issue by linking ecological observations to physical processes (i.e. coastal protection) on a geomorphic time scale. Nonetheless, the concerns raised here are of significant importance in terms of local management policies. Based on our findings, we recommend management practices on Bonaire and other Caribbean islands to focus on restoring or preserving the carbonate production potential and general resilience of Acropora and Orbicella reefs that actually have the potential to protect sensitive low-lying coastal areas. On Bonaire this would be the region from the town of Kralendijk to the southern tip of the island (i.e. LT cluster 2) where the coastal shore zone is already suffering from the effects of the loss of natural coral reef wave-breakers and where socio-economic implication will be greatest. Considering projections of increased frequency and intensity of physical disturbances (storms and large swell events) (Hoeke et al. 2013; Hopkinson et al. 2008), restoring reefs as natural coastal protection may be substantially more sustainable and cost-efficient than artificial coastal protection efforts.

The bulk of calcium carbonate that is removed biologically on Bonaire reefs can be attributed to parrotfish grazing. Our results suggest that parrotfish erosion is higher on structurally complex reefs with high coral cover. Although this appears contradictory, it is not surprising that more complex reefs harbour higher fish densities (Newman et al. 2015). By converting substrate into sand, parrotfish are an essential component ensuring reef stability through cementation. This is, to a lesser extent, also true for sand production by macro and microeroders. Currently, their contribution to gross bioerosion is limited, but benthic bioerosion is likely to increase when more substrate becomes available and water quality declines further (Schönberg et al. 2017; Marlow et al. 2018). Cluster 4 of the lower terrace appears to be exemplary to this development that will further undermine reef stability. The reef flat in this area receives considerable amounts of terrestrial run-off following precipitation and outflow from salina's. Substrate is widely available and indeed the highest excavating sponge densities were found in the survey. This is also a region that is relatively exposed and received considerable impact from recent hurricanes Lenny (1999) and Ivan (2004) that passed north of Bonaire. It is likely that the low 3D-complexity, low coral cover and large rubble walls are still residue of this impact (Bries et al. 2006).

It is noteworthy that the shallow reef zone in front of low-lying coastlines, which is nowadays mostly covered by sand and rubble, was formerly dominated by dense communities of Acropora spp. (Bak 1977; Van Duyl 1985). These fast-growing corals contribute disproportionally to reef accretion (Figure 5.4) and structural complexity, facilitating bottom roughness and therewith diminish wave energy at the coastline (Alvarez-Filip et al. 2011a). Consequently, the Caribbeanwide Acropora mass mortality has significantly reduced the wave-breaking capacity of the shallow reef zone on many Caribbean islands (Gladfelter 1982; Alvarez-Filip et al. 2011a). Whilst there appears to be a widespread perception that the 


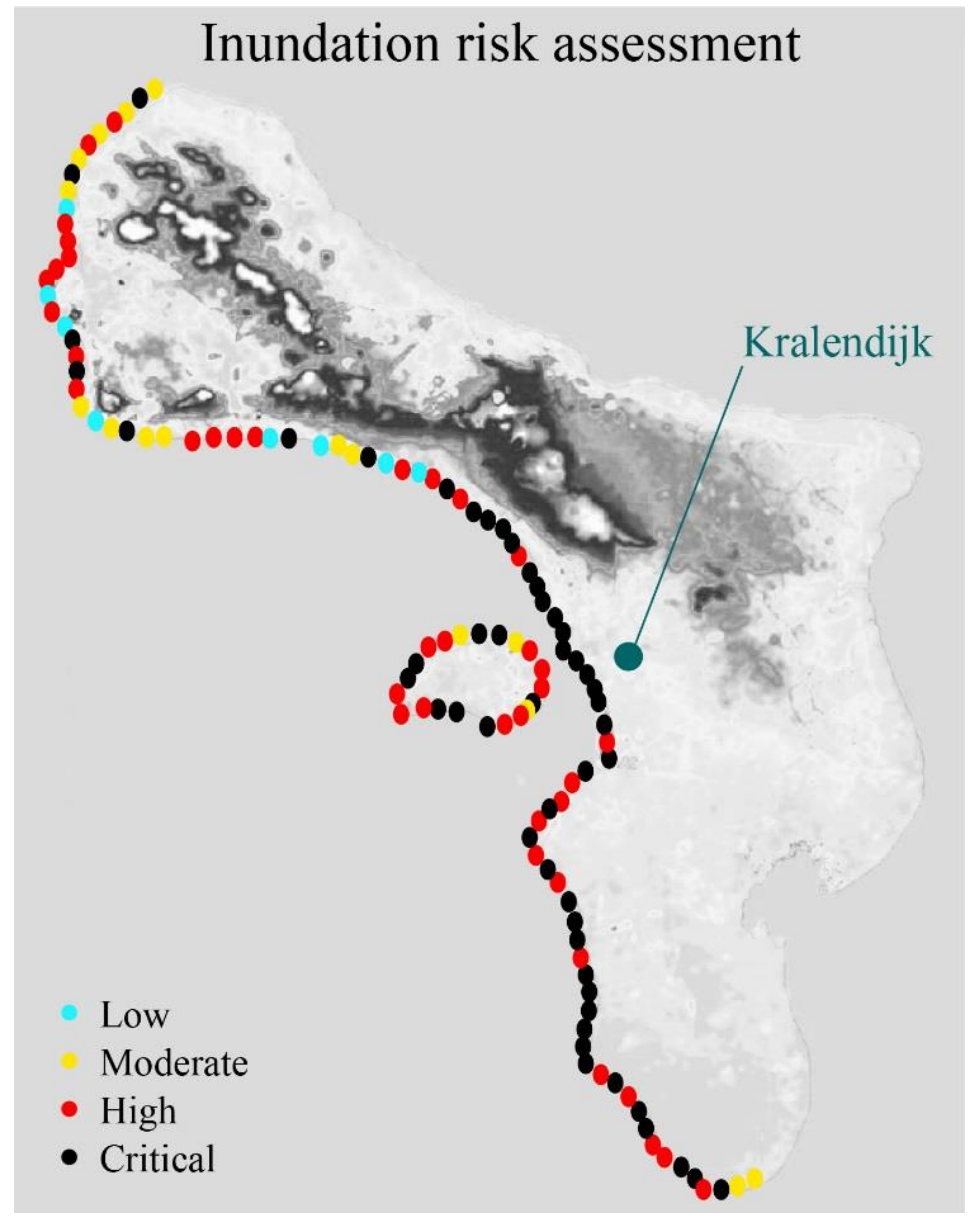

Figure 5.6. Map of Bonaire showing regions at high risk of flooding in case of extreme weather events on the leeward side of the island. The following parameters were included in the risk assessment: reef structural elevation from the bottom (reef height), height of the coastline, maximum reef accretion potential, width of the terrace and wind exposure. Colours indicate risk levels ranging from low to critical. The location of the Kralendijk is indicated on the map.

Bonaire coral reefs are relatively 'healthy', the loss of these principal calcifiers, declined coral cover and the current overall marginal reef accretion as presented here suggests that this might be a statement subjected to the 'shifting baseline syndrome' (Pauly 1995; Johnson and Jackson 2015; McClenachan et al. 2017). Nonetheless, structurally complex DO reefs dominated by framework-building Orbicella spp. do still exist around Bonaire, mostly in the northern region. These reefs were characterized by high coral cover and are comparable to the relatively 'good' reefs as described by Steneck et al. (2013) for Bonaire. Although these reefs were an exception 
rather than rule, their existence does indicate the potential of these reefs to continue to prevail under the currently unfavourable global environmental conditions. The observed variability in maximum reef accretion potential along the coastline implies that the local conditions, defined both naturally (Bak 1975) and through anthropogenic impact, can have a significant impact on the capacity of a reef to sustain vertical growth. Local biophysical drivers such as the degree of exposure, terrace width, community composition and colony morphology can greatly impact a reef's carbonate budget (Perry et al. 2015b) which indeed appears to be the case for Bonaire as well as Klein Bonaire. The most structurally developed Orbicella reefs, with generally high biodiversity and vertical growth (Mumby et al. 2008), were found in the region with the most narrow terrace and a cliff-like coastal morphology. This area, located north of Kralendijk (LT cluster 4, DO cluster 3 and 4), is also relatively unexposed, whereas high exposure has been found to constrain Orbicella reef distribution (Chollett and Mumby 2012). Most noteworthy, however, may be that this region receives relatively little local human disturbance. Within this region, near-pristine Orbicella reefs are found, some even with growth rates that can still track the more pessimistic sea-level rise projections (Sommer et al. 2011; Perry et al. 2013; this study). Here, markedly high levels of gross carbonate production are found in the marine reserve, where entrance by humans is prohibited (Koning Willem-Alexander no-dive reserve). Arguably, the marine reserve is located in a region with naturally favourable conditions, yet the coral cover and rugosity were higher in the reserve compared to the directly adjacent reef sites. Contrarily, the reefs in front of Kralendijk, and explicitly the lower terrace, receive extensive direct anthropogenic impact. This has led to a near complete functional collapse of the reef system in this area. These findings underline the impact of variability in local conditions and support the claim that locally focussed conservation efforts are essential to ensure that reefs maintain a net accretion rate that matches future sea-level rise.

\section{Methodological considerations}

By excluding the most shallow reef zones (<5 m depth) it is likely that we missed some important processes in terms of wave breaking and coastal protection (Harris et al. 2018). Ferrario et al. (2014) does, however, show that the fore-reef (reef crest and reef flat) already substantially diminish wave energy (> 90\%). Yet, a clear reef crest is largely absent on the leeward side of the Bonaire suggesting that the reef flat, including the lower terrace, former Acropora palmata zone and shore zone (Bak 1977), is by large responsible for dissipation of wave energy. The concerns expressed in this study may, therefore, apply less obviously to every day wave action, which is primarily deflected in the shallowest zones (A. palmata zone and shore zones), but more notably to extreme events (e.g. hurricanes, unusually large swell) that penetrate to depths of over 5 meters (Ferrario et al. 2014; Storlazzi et al. 2018). The impact of both forces will indeed be intensified by reduced structural complexity and rising sea levels. Their effects, however, will be observable on substantially different geological time scales (Kleypas et al. 2001). Extreme events are at present already having a considerable effect on the coastal region, while frequent inundation through every day wave action will, to a greater extent, be driven by the rate of SLR (Ferrario et al. 2014; Harris et al. 2018). 
It should also be noted that the methods applied in this study (ReefBudget and RAP) have their limitations. Naturally, the applied methodologies rely largely on assumptions made based on the available literature. The effect of sand transportation and dissolution (Eyre et al. 2018; Yates et al. 2017), rubble fields preventing sand transportation and rubble deposition onto shore (Engel et al. 2012), deposition and the geochemical and microbiological processes of lithification, the overall rising sea bed in this region or the impact of sub-rubble communities are by large ignored. Sub-rubble communities, for instance, are often dominated by CCA (Gischler 1997; Meesters et al. 1991). While such overlooked constituents can have an important role in net reef accretion and sea floor elevation (Yates et al. 2017) it is unlikely that including these will amend the worrying conclusions drawn here on the state of some of the best reefs in the Caribbean. Yet we encourage the integration of such relevant aspects into existing methodologies to quantify reef accretion. 
Chapter V

\section{Acknowledgement}

We would like to sincerely thank C. Eckrich for her support in the field. We also acknowledge the invaluable logistical assistance of the STINAPA Bonaire National Parks Foundation. This study has been funded by the Ministry of Economic Affairs for the purposes of the Policy Supporting Research Theme 'Analyse fotomateriaal koraalrif/fase 1' (Project No. BO-11019.02-038). The Royal Netherlands Institute for Sea Research provided funding of expenses and in-kind support. 


\section{Appendix 5.A}

Tree size selection graphs for the multivariate regression tree of the carbonate budget data for the lower-terrace (A) and drop-off (B) zones. Green circles represent the relative error which decreases with tree size, while the blue circles indicate the cross-validated relative error. Orange circle highlights the best tree, while the red circle shows the smallest tree within one standard error of the best tree. Blue vertical bars indicate one standard error and the solid red line represents one standard error from the lowest cross-validated relative error. Both graphs show a range of trees (A: 1-6, B: 5-9) that predict only marginally worse than the best tree (De'ath 2002). Multivariate regression tree for the carbonate budget data of the lower terrace (C) and drop-off zone (D). The individual budget constituents were standardized to the mean and standard deviation. Bars in shades of blue represent the multivariate constituents mean at each node. Each node is constrained by a threshold of latitude or longitude (lat, lon). Cluster numbers (c..) are given below the Bars, with (n) referring to the number of sites within each cluster. Residual error, cross-validated error and standard error are given at the bottom of the plot. Selection graphs and multivariate trees were created using the Mvpart package (De'ath 2006) in the R programming environment v3.4.1 (R Core Team 2013).

A

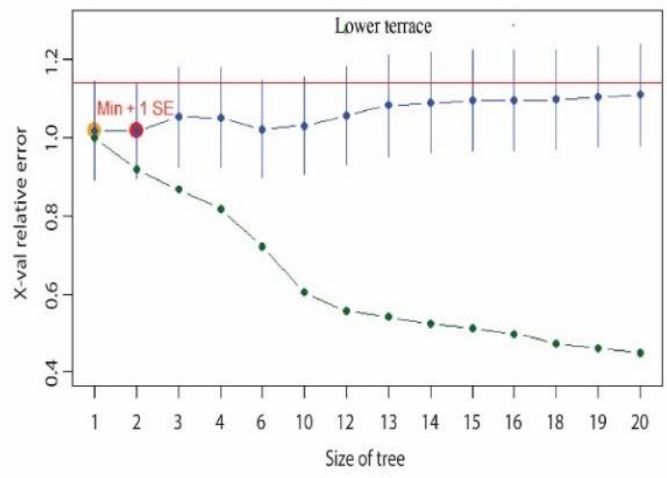

C

- CCA production

- Coral production

- Parrotfish erosion

Microbioerosion

- Urchin erosion

- Sponge erosion

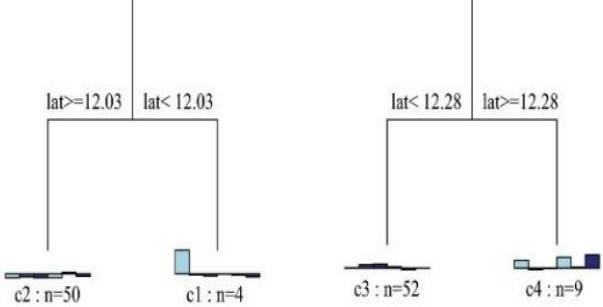

B

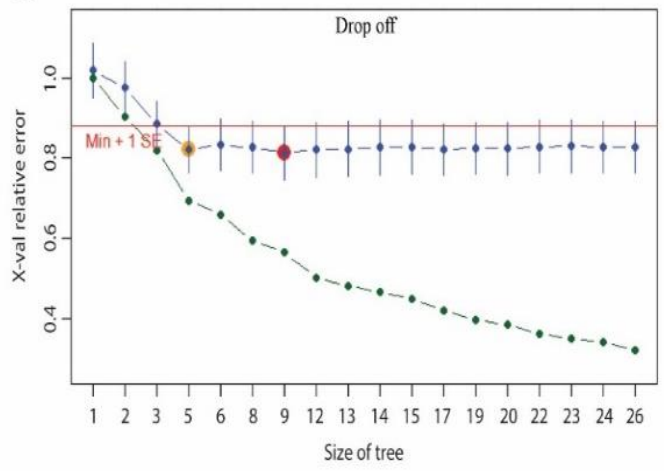

D

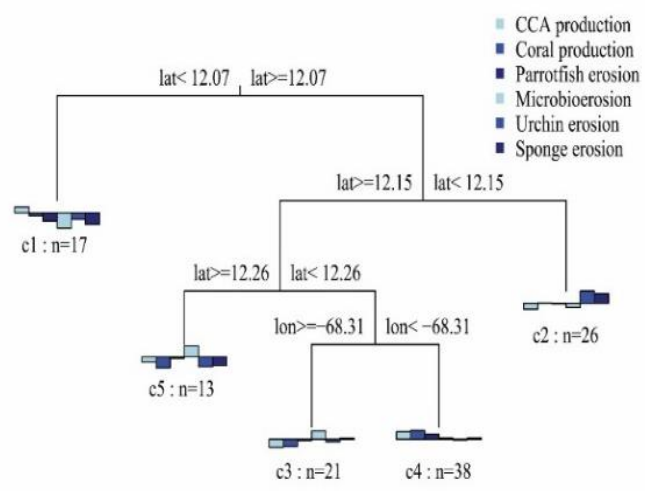




\section{Appendix 5.B}

Summary of reef characteristics for the different MRT clusters. Displayed values are means of all transects within clusters with their respective 95\% confidence limits. All production and erosion constituents are given in $\left(\mathrm{kg} \mathrm{CaCO}_{3} \mathrm{~m}^{-2} \mathrm{y}^{-1}\right)$. Substrate refers to the entire 3D surface (m) minus sand, i.e. all available hard substrate and substrate covered by living biota. 'Reefbuilding corals' represents the percentage of the total area covered by framework-building corals (sensu De Bakker et al. 2016b, but also including 'competitive' species) as opposed to opportunistic species within the coral community. The summary table can be accessed through the following link.

https://doi.org/10.18174/472452

\section{Appendix 5.C}

Local site characteristics and an overview of the data collected for each individual transect ("Data sheet"). Details on the various coral community, gross production, gross erosion, net production and vertical reef accretion metrics are provided in the "Info sheet". The overview table can be accessed through the following link.

https://doi.org/10.18174/472452 
N.t.

D.

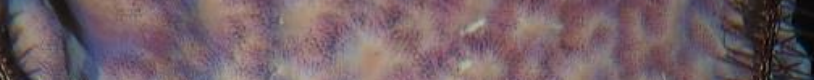

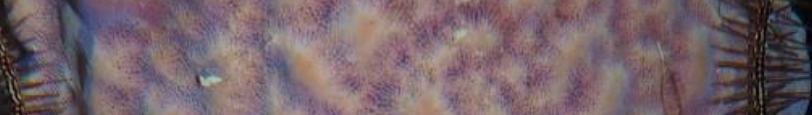

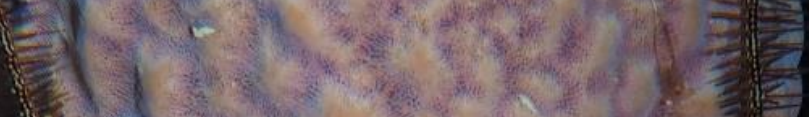

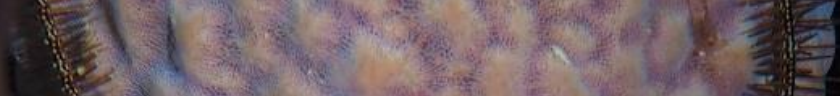

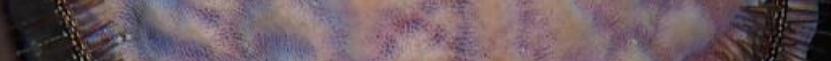

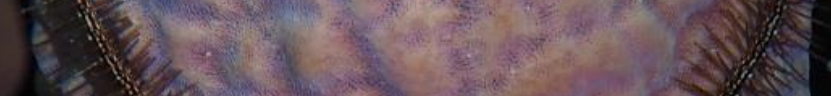

3.

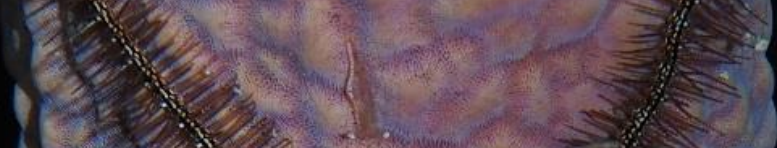

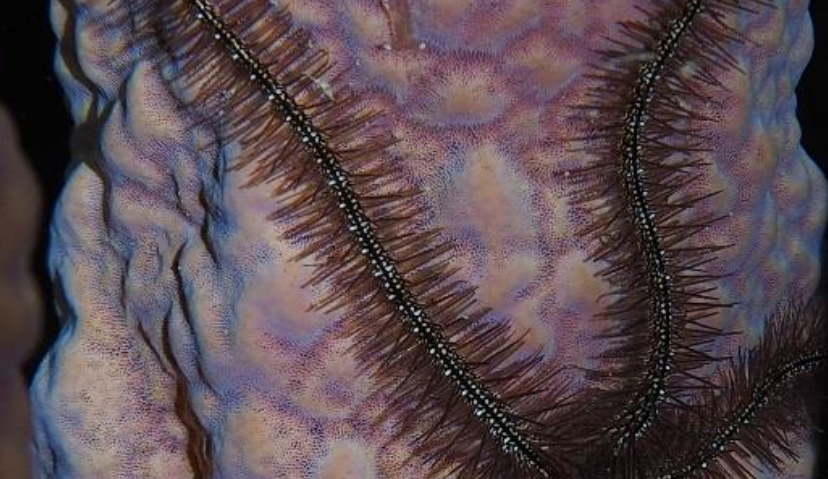

$(19)$

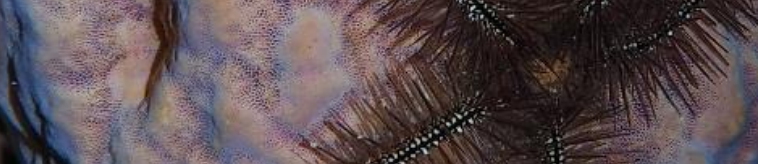

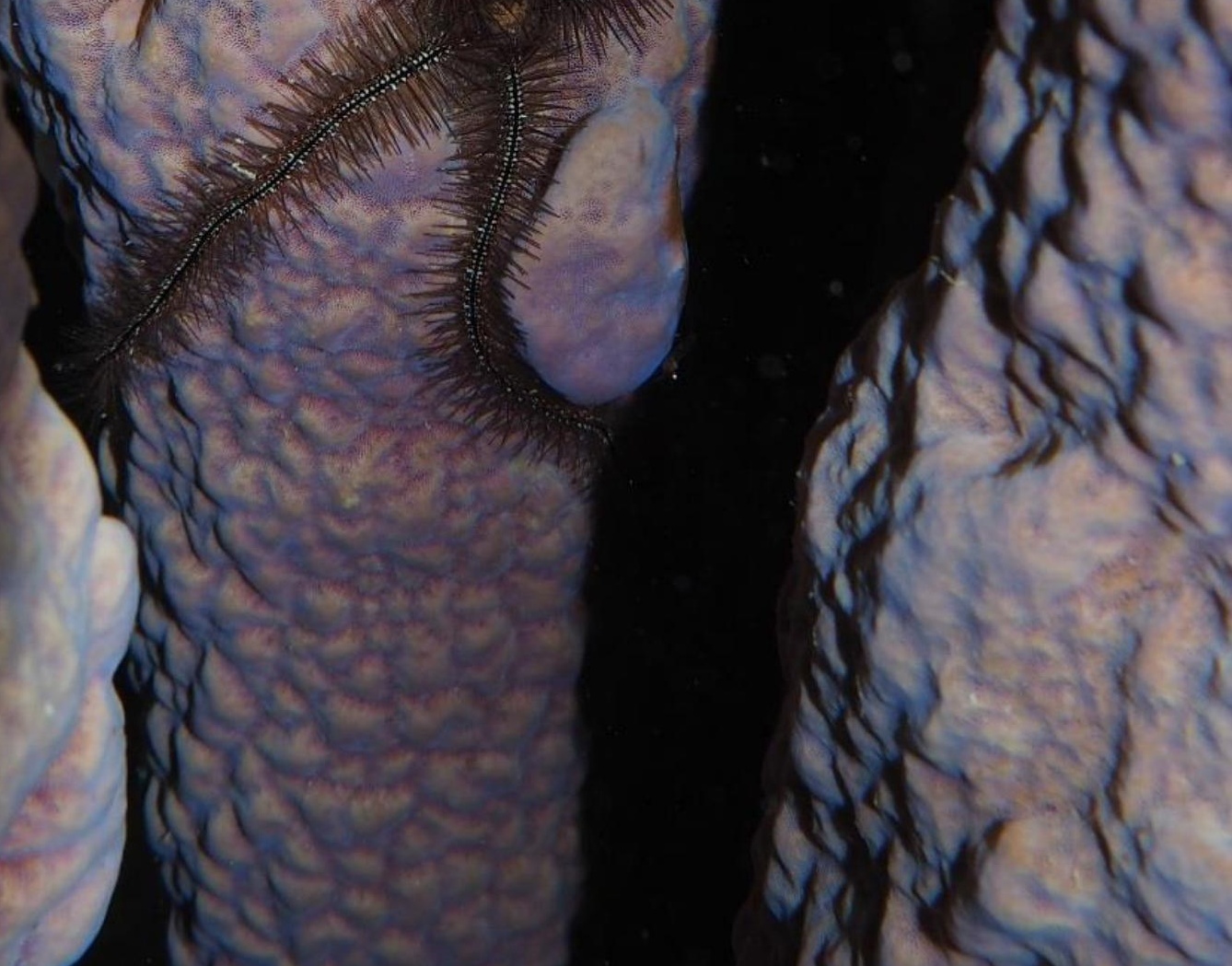




\title{
CHAPTER VI
}

\section{Towards stabilization of the decline in coral cover and fish biomass on the shallow reefs of Bonaire, Netherlands Caribbean?}

\author{
Didier M de Bakker, Fleur C van Duyl, Evert de Froe, Sil Piek, Estefania Velilla, Sarah SJ \\ Veillat, Yun JH Scholten, Sofie Nietzel, Roger Meijs, Fee Zanke, Alice E Webb and
} Erik H Meesters

\begin{abstract}
The coral reef around Bonaire, Netherlands Caribbean, is often considered to be in a good ecological condition relative to the wider Caribbean region. Yet, descriptions of high cover of corals, low abundance of macroalgae and substantial fish stocks may be restricted to regions with little anthropogenic activity. We conducted surveys across the entire leeward fringing reef (115 sites) in 2014 and 2017 examining the status of benthic assemblages and reef fish communities in the reef terrace zone (5-7 $\mathrm{m}$ depth) and drop-off zone (8-12 $\mathrm{m}$ depth). Elaborated survey efforts on such a fine spatial scale revealed considerable variation in reef 'health' that could obviously be linked to the intensity of human activity in the adjacent coastal area. We described configuration of typical reef communities along a gradient of human impact that are nowadays recognizable on these Caribbean reefs. Despite the finding that many reef sites show clear signs of ecological decline, the presented data also confirms the 'survival' of near-pristine reefs. It appears that, when local conditions are favourable, these reefs have the capacity to endure, even under the current levels of harsh global perturbation (i.e. environmental change). More notably, a comparison of reef condition among years hints towards a stabilization of the decline in coral cover and fish biomass on the shallow reef. Although this study can, to date, only tentatively support this hypothesis due to the relatively short period studied, comparison to previously conducted surveys appears to support such a progressive development.
\end{abstract}




\section{Introduction}

Over recent decades, many Caribbean coral reef ecosystems have suffered severe large-scale ecological degradation threatening ecological functioning and trophic flows (Hughes et al. 2003; Pandolfi et al. 2003; Hughes et al. 2017). The changing global environment in combination with local human impacts has reduced water quality and enhanced conditions in which pathogens and coral bleaching prevail (Nyström et al. 2000; Aronson and Precht 2001; Wooldridge 2009; Vega-Thurber et al. 2014). Synergistically, overexploitation of herbivorous fish and the massmortality of Diadema antillarum sea urchins severely diminished grazing pressure on reef algae (Bak et al. 1984; Jackson et al. 2001; Jackson et al. 2014). Consequently, major shifts occurred in benthic sessile communities in favour of fast-growing opportunistic functional groups such as algal turf assemblages, fleshy macroalgae and cyanobacterial mats (Hughes 1994; Fabricius et al. 2005; Vermeij et al. 2010; De Bakker et al. 2017; Ford et al. 2018). The altered conditions on the reef have provided these organisms a competitive advantage at the expense of scleractinian corals and other ecologically relevant organisms. Simultaneously, the densities of most reef fish clades, including the aforementioned herbivores (parrotfish, surgeonfish), groupers, snappers and large carnivores, declined profoundly (Paddack et al. 2009).

Although all Caribbean reefs are affected by anthropogenic disturbance, an apparent gradient exists in the state of ecological degradation from nearly pristine reefs to reefs that are at the tipping point of full functional collapse (Jackson et al. 2014; Smith et al. 2016). The leeward fringing reef of Bonaire (southern Caribbean) is generally considered to be among the least regraded in the region (Steneck et al. 2013; Jackson et al. 2014). Due to its geographical position in the southern Caribbean, hurricanes and tropical storms rarely impact the island (Lugo et al. 2000; Bries et al. 2004), allowing for extensive reef formation (Bak 1977; Van Duyl 1985). Human activity on Bonaire is generally focussed on the leeward side of the island and concentrated around the capital (Kralendijk) (De Meyer et al. 1998). While Bonaire is not densely populated the number of inhabitants has expanded rapidly over the past decades (CBS 2018, cbs.nl), amplifying pressure on reef benthos and local fish stocks. Bonaire established a National Marine Park (BNMP) in 1979 covering an area of $27 \mathrm{~km}^{2}$, extending from the shoreline down to $60 \mathrm{~m}$ depth. In addition, two fish reserves (no fishing allowed) and two other reserves (no diving or snorkelling allowed) were established in 2008.

The coral reefs of Bonaire are often characterized by higher than average coral cover, little prevalence of diseases, low macroalgal abundance, and relatively high fish densities (Kramer 2003; Steneck et al. 2013; De Graaff et al. 2016). Yet, various studies indicate reef declining trends to occur on these reefs as well (Stokes et al. 2010; Sommer et al. 2011; De Bakker et al. 2017). These studies point out that the benthic community composition around Bonaire and the neighbouring island of Curaçao has changed drastically on large sections of the reef. Simultaneously, various groups of fish (e.g. parrotfish, groupers, snappers, sharks) have gradually declined in size and abundance due to increased fishing pressure and the loss of structural relief (Wilkinson 2004; Perry et al. 2012; Newman et al. 2015; De Graaff et al. 2016). 
Overall, the available literature appears to reflect contradicting views on the present-day condition of the fringing reef surrounding Bonaire (e.g. Steneck et al. 2013 compared to De Bakker et al. 2017). Such disparity may principally be incited by variations in the local natural conditions and the degree of human induced disturbance at the studied reef sites. While spatial variation in the severity of reef degradation intuitively occurs among geographically distinct regions (Sandin et al. 2008b; Jackson et al. 2014; Smith et al. 2016), the fallout of variations in the intensity of disturbances is expected to occur on much smaller spatial scales as well (e.g. intra-island) (De Bakker et al. 2016b). An elaborate study determining the reef accretion potential across the complete leeward reef of Bonaire confirms that strong spatial variation can indeed exist on an island scale (Chapter V). The apparent uncertainty on the status of the reefs of Bonaire calls for a thorough reassessment of the current ecological status at high spatial resolution. The localized variation in anthropogenic disturbance (from virtually absent to high impact) in combination with the designated marine park and reserves (Figure 6.1), give the leeward fringing reef of Bonaire the ideal gradient to examine spatial variation in present-day benthic and reef fish assemblages.

In this study, we evaluate the current status of the shallow coral reef ( $<15 \mathrm{~m}$ depth) along the leeward side of Bonaire. The assessment was based on quantitative benthic sessile and reef fish community surveys, conducted in 2014 and 2017. Thereby explicitly investigating the potential of small-scale spatial and temporal variation. Additionally, the benthic surveys are used to reconsider the classification of typical benthic habitats encountered in the shallow Caribbean reef ecosystem. Classical baseline descriptions of Caribbean coral reefs will in many cases not be appropriate anymore to the current situation (Knowlton and Jackson 2008) and returning to past configurations seems almost infeasible (Hughes et al. 2017). Hence, a classification is proposed describing distinct benthic assemblages that developed under a range of anthropogenic impact.

\section{Materials and Methods}

Extensive benthos (macrofauna and macroflora) and reef fish surveys were conducted in the shallow fringing reef habitat of the southern-Caribbean island of Bonaire. Surveys were limited to the leeward side of the island, where reefs and human activities are concentrated. A total of 115 sites were assessed, separated by an a priori set distance of $500 \mathrm{~m}$ and covering the leeward reef of Bonaire and satellite island of Klein Bonaire $\left(\sim 6 \mathrm{~km}^{2}\right)$ (Figure 6.1 \& Appendix 6.A). At each site, data were collected of benthos and fish communities in the lower terrace zone (LT, 5-7 m depth) and the drop-off zone (DO, 8-12 m depth) along fixed 25-m transect lines (2 per zone) positioned parallel to the orientation of the reef front and separated by $10 \mathrm{~m}$. At sites where the zones differed from the characteristic depth (Bak 1977), we decided to place transects inside the reef zone rather than sticking to the specific depth range (at 15 sites in the northern region and on Klein Bonaire the DO zone was found at $\sim 15 \mathrm{~m}$ ). Surveys were conducted between September and December 2014 and over the same period in 2017. 


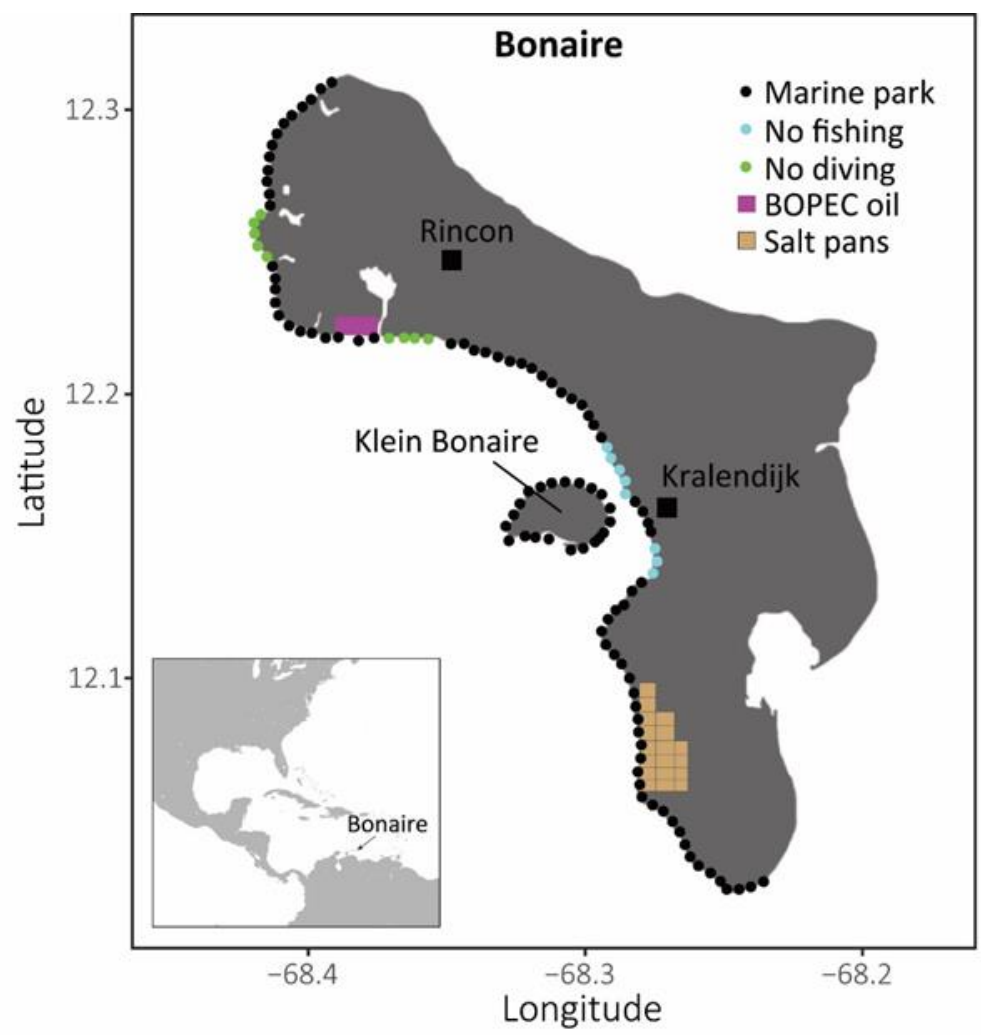

Figure 6.1. Overview of Bonaire and its location in the wider western Atlantic. Points indicate the position of the 115 surveyed reef sites on the leeward side of Bonaire, including two no fishing zones (blue) and two no dive reserves (green). Black squares mark the centre of Kralendijk (capital) and Rincon. The BOPEC oil storage (pink) company and saltpans (yellow) are indicated on the map as well.

\section{Benthic community structure}

Benthic sessile communities were assessed at the last $10 \mathrm{~m}(15-25 \mathrm{~m})$ of both transect lines in each zone. The contribution of each benthic component (both biotic and abiotic) was quantified in situ as the total distance (to the nearest $\mathrm{cm}$ ) covered directly below the main transect line. Benthic organisms were identified in situ and to the finest taxonomic resolution (i.e. to species level for corals, to genus level for most macroalgae, etc.). For general site characterization, all encountered benthic components were grouped in major functional categories, as defined by De Bakker et al. (2017) (Table 6.1). The cover of all categories was standardized to the mean and standard deviation (Faith et al. 1987) for further analyses. Site partitioning was achieved by comparison of the composition (mean cover) of the ten most prominent biotic and abiotic categories (Table 6.2) through Euclidian distance, followed by single linkage agglomerative clustering (Legendre and Legendre 2012; Wilks 2011). We refrained from including a contiguity component as spatial explanatory variable (see Chapter V) in the cluster analysis (e.g. Legendre et al. 1990) because the distribution of benthic communities may not always show a clear spatial 
sequence and can vary substantially over limited distance. Ward's minimum variance method (Ward 1963) was selected as best clustering model through the cophenetic correlation (Pearson's $r$ ) (Sokal and Rohlf 1962) between the original dissimilarity matrix and the cophenetic matrix. Ward's hierarchical clustering relies on defining clusters with the smallest within-group sum of squares and is commonly used as an exploratory tool in ecology studies (Borcard et al. 2011; Wilks 2011). The optimal number of distinct communities (clusters) was determined based on the cut with the highest matrix (Mantel) correlation computed through comparison of the distance matrix to binary matrices cut at different levels (Legendre and Legendre 2012; Maechler 2018) (Appendix 6.B). Final community characterization was derived from the acquired classification through indicator species (here functional groups of organisms and abiotic substrates) analysis (i.e. reef communities were specified by the community constituent with a significant indicator value) (Roberts and Roberts 2016). Community assessment was conducted separately for the lower terrace zone and the drop-off zone due to obvious dissimilarity in community composition. Non-metric multidimensional scaling (nMDS) was applied to visually confirm clustering.

To distinguish distinct coral community compositions, the same clustering approach as delineated above was applied to a dissimilarity matrix based on the standardized (to the mean and standard deviation) mean cover of all coral species encountered at each site. For the coral community clustering, zones were analysed together because specific coral assemblages were encountered in both zones.

To examine short-term temporal variation in the composition of benthic communities BrayCurtis dissimilarity values were calculated based on square-root transformed mean cover of the main benthic categories. We then applied permutation-based multivariate analysis of covariance (PERMANOVA) (Anderson 2001) to test whether benthic community composition differed between the 2014 and 2017 (99 999 permutations). Linear regression was used to model the temporal change in cover of the individual benthic categories between 2014 and 2017 and how that change varied between the two zones (LT and DO). Assumptions for linear regression were confirmed visually (Zuur et al. 2007).

\section{Specific reef characteristics.}

In addition to the basic assessment of benthic cover, various facets related to the condition of the reef were examined as well. Coral recruitment potential was determined as the number of recruits $(\leq 40 \mathrm{~mm})$ found at each site in 2017. Recruits were counted in $25 \mathrm{~cm}^{2}$ quadrats positioned every two meters directly adjacent to the transect line (six quadrats per transect). The abundance and size (test diameter) of Diadema antillarum sea urchins was recorded within 1 meter on both sides of the transect $\left(20 \mathrm{~m}^{2}\right.$ in total). Finally, presence of the colonial tunicate Trididemnum solidum, formerly destructive at several Bonaire reef sites (Bak et al. 1981; Sommer et al. 2010), was scored when encountered covering surface under the main $10 \mathrm{~m}$ transect line. These parameters were only included in the survey in 2017 and as such, data for 2014 is not available. Structural relief (rugosity) of the bottom was defined by the difference in length of the true surface profile of the reef under the benthic transect line and the benthic transect line (10 m) itself. Rugosity data was collected in both 2014 and 2017. 


\section{Reef Fishes}

Body size (fork length) and numerical densities of all reef fish was recorded within a 5-m wide belt transect centred on the main 25-m transect lines. Each transect was surveyed twice, once to count conspicuous species and a second time to count demersal and cryptic species. To ensure consistency, all fish surveys were conducted between at least $2 \mathrm{~h}$ after sunrise and 2 hours before sunset (Harmelin-Vivien et al. 1985; Newman et al. 2006). The encountered fish were identified to species level or lowest recognizable taxon (Appendix 6.G). All species were assigned to one of three functional trophic guilds following Pratchett et al. (2011): carnivores, herbivores and omnivores. Groupers and Snappers were assigned to a subcategory as "commercially important" species following the Healthy Reefs Initiative (http://www.healthyreefs.org). The weight of each individual fish was calculated based on the estimated length using the equation as defined by Bohnsack and Harper (1988) (Equation 6.1).

$$
W=a L^{b}
$$

Where $W$ is the weight of the fish calculated from the fork length (L) and constants $a$ and $b$.

Species specific values for constants $a$ and $b$ were taken the Atlantic and Gulf rapid reef assessment (AGRRA: http://www.agrra.org/) (for constants per species see Appendix 6.C). Temporal and spatial variation in fish communities was investigated through linear regression.

\section{Statistical analyses}

All statistics were conducted in the $\mathrm{R}$ programming environment. Within $\mathrm{R}$, the following packages were used: 'stats' (R Core Team 2002), 'vegan' (Oksanen et al. 2013), 'cluster' (Maechler 2018), 'labdsv' (Roberts and Roberts 2016).

\section{Results}

\section{General benthic reef characteristics}

Testing of temporal change indicates that there was no apparent difference in the overall benthic community composition between 2014 and 2017 in both zones (PERMANOVA, $F=1.33, p=$ 0.24). Univariate analysis of the cover per benthic group, however, did show some temporal variation (Table 6.1). An overall decline was observed in the cover of algal turfs and benthic cyanobacteria. Compared to 2014, algal turfs appear to have receded relative to bare rubble, gorgonians, hydrocorals and macroalgae in 2017. Despite the apparent decline, algal turf assemblages were still the dominant biotic functional reef component, generally covering the majority of the hard substratum on the shallow reefs of Bonaire in 2017. Their reduced abundance appears to have little to no effect on scleractinian coral cover. 
Most notably, the cover of scleractinian corals did not decline significantly in either of the studied zones (Table 6.1). Granting that scleractinian corals were the second most dominant group of organisms (Table 6.1), their mean cover has over time been reduced to a low but, at least since 2014, stable $12 \%$. Coral cover in the drop-off zone was substantially higher than in the lower terrace zone (6\%) and still averages on approximately $19 \%$. Highest coral cover was found north of Kralendijk (capital), with the exception of the more exposed northernmost region. Substantial coral cover was also observed in the southernmost tip of the island, several sites on Klein Bonaire and in both no-dive reserves. Scleractinian corals were exceptionally abundant in both reserves (Figure 6.1 \& Appendix 6.D) covering on average 15.2\% (95\% CI: 8.9-23.2) in the lower terrace zone and $29.6 \%$ (95\% CI: $24.3 \%-35.4 \%$ ) in the drop-off zone. Crustose coralline algae (CCA) and hydrocorals (Milleporidae) exhibited similar patterns of distribution to scleractinian corals, although cover of these species was also relatively high in some of the far northern and other typically more exposed sites (Appendix 6.D). In 2017, gorgonian densities were highest on reefs on the southern region of Bonaire, a stretch of reef just north of Kralendijk and the western sites on Klein Bonaire (Appendix 6.D). Cover of the formerly described organisms (excl. algal turfs), which can be considered indicators of considerable good reef health, was overall lowest in front of the densely populated town of Kralendijk. Concurrently, the cover of benthic cyanobacteria, albeit found in low densities across the shallow reef, and sponges was fairly high in this region, most notably in the vicinity of water inlets (e.g. the harbour). Fleshy macroalgae are virtually absent on the shallow reef of Bonaire. Yet, substantial macroalgal stands (mostly Dictyota spp. with some Sargassum spp.) were found in both zones at the northernmost sites. Sand and rubble nowadays account for a substantial fraction of the total reef surface, principally in the lower terrace zone and most pronounced in front of Kralendijk (Appendix 6.D).

\section{Benthic reef community classification}

Based on the ten major (a)biotic reef components (given in Table 6.2), four distinct benthic reef communities were distinguished in the lower terrace zone as well as in the drop-off zone (Figure 6.2). Characterization was based on the significance of the indicator-values and mean cover of the main components (Table 6.2). A description of each benthic community is given below, community characteristics are shown in Table 6.2. Similar communities were encountered in both zones but communities were generally more diverse in the drop-off zone (e.g. Table 6.3). Here, generally more hard substratum was available for organisms to colonize, often resulting in higher cover of corals and other typical reef biota (including sponges, gorgonians, Milleporidae and CCA). Cluster analyses resulted in a highly comparable arrangement of benthic communities along the leeward coast and around Klein Bonaire for both 2014 and 2017. As such, only the results of 2017 are presented in this specific assessment (Figure 6.2 \& Table 6.2). 
Table 6.1. Benthic community composition. Mean percentage cover of the various functional benthic groups in 2014 and 2017. Means are presented for the lower terrace zone, the drop-off zone and for the entire shallow reef of Bonaire (total). 95\% confidence limits are given in brackets. Bold values indicate a significant (at the 0.05 level) temporal change in cover of the specific group based on linear regression. The abiotic 'Rubble' component could be covered by turf, benthic cyanobacterial mats (BCMs) or crustose coralline algae (CCA) or other taxa. Similarly, sand could be covered by benthic cyanobacteria. In these cases, cover was included as both the biotic and abiotic component.

Lower terrace zone

Drop-off zone

Total

\begin{tabular}{|c|c|c|c|c|c|c|}
\hline Biotic & 2014 & 2017 & 2014 & 2017 & 2014 & 2017 \\
\hline Scleractinian corals & $\begin{array}{c}7.06 \\
(5.79-8.46)\end{array}$ & $\begin{array}{c}6.25 \\
(5.05-7.58)\end{array}$ & $\begin{array}{c}18.96 \\
(17.65-20.32)\end{array}$ & $\begin{array}{c}19.54 \\
(17.69-21.48)\end{array}$ & $\begin{array}{c}12.31 \\
(11.17-13.50)\end{array}$ & $\begin{array}{c}11.97 \\
(10.71-13.30)\end{array}$ \\
\hline $\mathrm{CCA}$ & $\begin{array}{c}0.47 \\
(0.28-0.69)\end{array}$ & $\begin{array}{c}0.85 \\
(0.55-1.22)\end{array}$ & $\begin{array}{c}3.75 \\
(3.18-4.36)\end{array}$ & $\begin{array}{c}1.96 \\
(1.60-2.36)\end{array}$ & $\begin{array}{c}1.72 \\
(1.41-2.05)\end{array}$ & $\begin{array}{c}1.35 \\
(1.09-1.63)\end{array}$ \\
\hline Algal turfs & $\begin{array}{c}47.15 \\
(43.05-51.43)\end{array}$ & $\begin{array}{c}31.63 \\
(27.60-35.92)\end{array}$ & $\begin{array}{c}40.34 \\
(38.48-42.24)\end{array}$ & $\begin{array}{c}34.57 \\
(32.17-37.05)\end{array}$ & $\begin{array}{c}43.67 \\
(41.44-45.95)\end{array}$ & $\begin{array}{c}33.08 \\
(30.69-35.56)\end{array}$ \\
\hline Macroalgae & $\begin{array}{c}1.05 \\
(0.66-1.51)\end{array}$ & $\begin{array}{c}4.25 \\
(3.00-5.73)\end{array}$ & $\begin{array}{c}3.28 \\
(2.57-4.08)\end{array}$ & $\begin{array}{c}7.14 \\
(5.91-8.48)\end{array}$ & $\begin{array}{c}2.01 \\
(1.60-2.46)\end{array}$ & $\begin{array}{c}5.60 \\
(4.67-6.62)\end{array}$ \\
\hline $\mathrm{BCMs}$ & $\begin{array}{c}0.12 \\
(0.05-0.22)\end{array}$ & $\begin{array}{c}0.11 \\
(0.04-0.21)\end{array}$ & $\begin{array}{c}4.25 \\
(3.48-5.10)\end{array}$ & $\begin{array}{c}1.70 \\
(1.33-2.11)\end{array}$ & $\begin{array}{c}1.46 \\
(1.14-1.81)\end{array}$ & $\begin{array}{c}0.67 \\
(0.51-0.85)\end{array}$ \\
\hline Gorgonians & $\begin{array}{c}0.17 \\
(0.10-0.26)\end{array}$ & $\begin{array}{c}2.35 \\
(1.64-3.19)\end{array}$ & $\begin{array}{c}0.86 \\
(0.65-1.11)\end{array}$ & $\begin{array}{c}4.93 \\
(3.91-6.08)\end{array}$ & $\begin{array}{c}0.45 \\
(0.34-0.57)\end{array}$ & $\begin{array}{c}3.52 \\
(2.88-4.23)\end{array}$ \\
\hline Hydrocorals & $\begin{array}{c}0.60 \\
(0.40-0.85)\end{array}$ & $\begin{array}{c}1.59 \\
(1.20-2.03)\end{array}$ & $\begin{array}{c}0.22 \\
(0.15-0.31)\end{array}$ & $\begin{array}{c}1.25 \\
(1.00-1.53)\end{array}$ & $\begin{array}{c}0.39 \\
(0.29-0.50)\end{array}$ & $\begin{array}{c}1.42 \\
(1.18-1.67)\end{array}$ \\
\hline Sponges & $\begin{array}{c}0.84 \\
(0.59-1.14)\end{array}$ & $\begin{array}{c}0.34 \\
(0.21-0.49)\end{array}$ & $\begin{array}{c}1.15 \\
(0.90-1.43)\end{array}$ & $\begin{array}{c}1.88 \\
(1.54-2.25)\end{array}$ & $\begin{array}{c}0.99 \\
(0.81-1.19)\end{array}$ & $\begin{array}{c}0.95 \\
(0.77-1.15)\end{array}$ \\
\hline Other biota & $\begin{array}{c}0.00 \\
(0.00-0.01)\end{array}$ & $\begin{array}{c}0.00 \\
(0.00-0.01)\end{array}$ & $\begin{array}{c}0.06 \\
(0.03-0.09)\end{array}$ & $\begin{array}{c}0.07 \\
(0.04-0.11)\end{array}$ & $\begin{array}{c}0.02 \\
(0.01-0.03)\end{array}$ & $\begin{array}{c}0.03 \\
(0.02-0.04)\end{array}$ \\
\hline \multicolumn{7}{|l|}{ Abiotic } \\
\hline Rubble & $\begin{array}{c}22.88 \\
(18.96-27.17)\end{array}$ & $\begin{array}{c}17.89 \\
(14.47-21.67)\end{array}$ & $\begin{array}{c}3.18 \\
(2.45-4.00)\end{array}$ & $\begin{array}{c}10.74 \\
(8.97-12.66)\end{array}$ & $\begin{array}{c}10.76 \\
(9.02-12.65)\end{array}$ & $\begin{array}{c}14.09 \\
(12.21-16.09)\end{array}$ \\
\hline Sand & $\begin{array}{c}12.60 \\
(9.91-15.62)\end{array}$ & $\begin{array}{c}12.02 \\
(9.26-15.13)\end{array}$ & $\begin{array}{c}6.22 \\
(5.17-7.36)\end{array}$ & $\begin{array}{c}6.68 \\
(5.37-8.14)\end{array}$ & $\begin{array}{c}9.13 \\
(7.77-10.59)\end{array}$ & $\begin{array}{c}9.16 \\
(7.69-10.75)\end{array}$ \\
\hline Pavement & $\begin{array}{c}0.02 \\
(0.01-0.05)\end{array}$ & $\begin{array}{c}0.24 \\
(0.08-0.49)\end{array}$ & $\begin{array}{c}4.32 \\
(3.49-5.24)\end{array}$ & $\begin{array}{c}0.00 \\
(0.00-0.01)\end{array}$ & $\begin{array}{c}1.24 \\
(0.95-1.58)\end{array}$ & $\begin{array}{c}0.07 \\
(0.03-0.14)\end{array}$ \\
\hline Recently dead coral & $\begin{array}{c}0.00 \\
(0.00-0.01)\end{array}$ & $\begin{array}{c}0.00 \\
(0.00-0.00)\end{array}$ & $\begin{array}{c}0.00 \\
(0.00-0.01)\end{array}$ & $\begin{array}{c}0.00 \\
(0.00-0.00)\end{array}$ & $\begin{array}{c}0.00 \\
(0.00-0.01)\end{array}$ & $\begin{array}{c}0.00 \\
(0.00-0.00)\end{array}$ \\
\hline
\end{tabular}

\section{Benthic communities on the lower terrace}

\section{Rubble-Turf}

The Rubble-Turf community dominates the lower terrace zone (65 sites) on the leeward side of Bonaire and on Klein Bonaire, with the exception of the northern region (north of the BOPEC) (Figure 6.2). Algal turf is the biotic indicator component in this community, while the abiotic substratum predominately consists of coral rubble (predominantly Acropora rubble, often overgrown by turf) and turf covered dead coral colonies or pavement (Table 6.2). Sandy patches accounted, on average, for $11.5 \%$ of the bottom. Live coral colonies are sporadically found in this community, resulting in a mean live coral cover of approximately $5 \%$ and a Millepora spp. cover of $1.5 \%$. Other benthic taxa together cover, on average, less than $3 \%$ of the total substratum. 


\section{Macroalgae-CCA}

This community composition was predominantly found in the relatively exposed northern region of Bonaire (Figure 6.2) and was characterized by dead coral substratum covered by substantial fleshy macroalgae stands (mostly Dictyota spp. with some Sargassum spp.) and relatively high cover of crustose coralline algae. Sand or rubble patches were relatively sparse, which may be linked to substantial water movement in this area (Table 6.2). Coral cover here lies around $8 \%$ and generally consisted of opportunistic species such as Madracis mirabilis, Pseudodiploria spp. and Porites astreoides (Figure 6.3). Other taxa, with the exception of algal turfs $(\sim 11 \%)$, and abiotic substratum (rubble, sand) were scarce. This type of community was also encountered in the exposed south eastern-most site.

\section{Coral-Gorgonian}

This is the sole lower terrace community with substantial live coral cover $(\sim 14 \%)$, although algal turf covered substratum is still dominant on these reefs. The dominant corals within this community were often Orbicella spp., M. mirabilis, Pseudodiploria spp. (Figure 6.3) Besides corals, the biotic community at these sites is dominated by gorgonian stands (mostly sea-rods and sea-plumes). Other biota (hydrocorals, CCA, sponges, macroalgae and cyanobacteria) were also fairly abundant, suggesting that these reefs can harbour a relatively high biodiversity compared to the other three lower terrace communities. The coral-gorgonian reef assemblages were, however, encountered at only 16 sites (14\%). This community was found in the sheltered area north of the densely populated capital, towards the southern point of the island and at two sites on Klein Bonaire (Figure 6.2). 

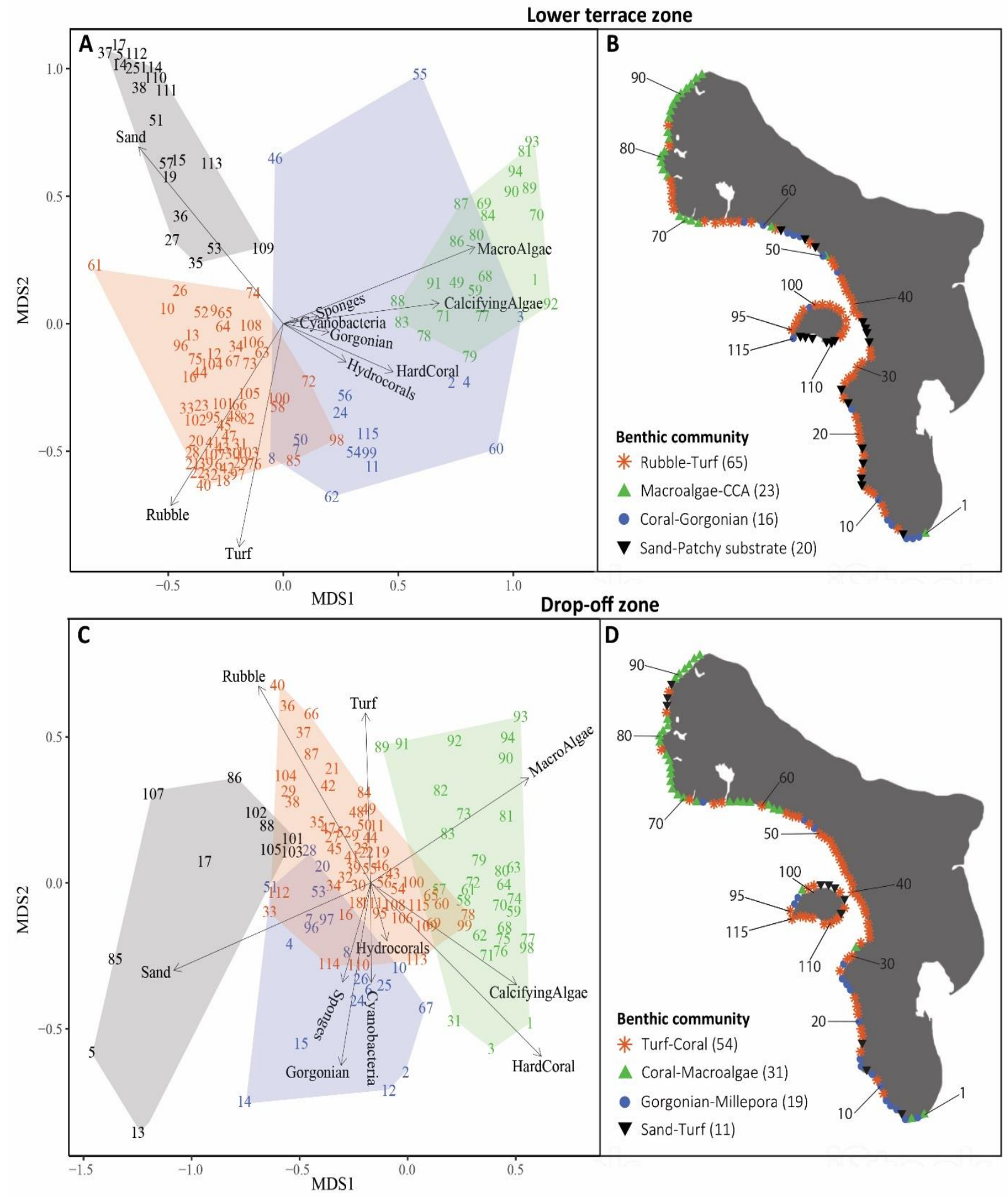
Figure 6.2. Benthic community clustering and arrangement of communities along the leeward coast of Bonaire in 2017. Clustering is based on the mean cover of the ten major (a)biotic functional groups at each site (115) and visualized through non-metric multidimensional scaling $(\boldsymbol{A}, \boldsymbol{C})$. Clustering was performed separately for the lower terrace zone (top panels) and the drop-off zone (bottom panels). Clusters are indicated by colours and, in the right panels, also by the shape of the symbols. Site numbers in the nMDS correspond to the site numbers on the map of Bonaire (B, D: enhanced numbers). Sites are numbered consecutively from south to north, with the exception of Klein Bonaire. Vectors in the nMDS indicate the direction of each community components. Number of sites in each cluster is given in brackets.

\section{Sand-Patchy substrate}

This arrangement consists almost entirely of sandy areas (62\% of the total cover) with sparse patches of substratum (Table 6.2). Algal turfs generally cover the little hard substratum that is present at these sites. Scleractinian coral and gorgonians both account for a mere $1 \%$ of the total cover, while all other biota together generally do not cover more than $0.5 \%$. This is the least biodiverse benthic community configuration in the lower terrace zone. Many reefs in front of the centre of Kralendijk are characterized by this composition. This arrangement is also found on the southern reefs of Klein Bonaire, a few sites to the north and south of Kralendijk and multiple sites in front of the saltpans (Figure 6.2).

\section{$\underline{\text { Turf-Coral }}$}

\section{Benthic communities of the drop-off zone}

The Turf-Coral community in the drop-off zone spatially coincides largely with the Rubble-Turf community of the lower terrace zone, although in the drop-off community coral cover was substantially higher $(\sim 20 \%)$. Rubble and sand patches were also less common compared to the LT zone. Rubble is likely less abundant because the DO zone was not dominated by Acropora spp. before the white band disease eradicated most colonies. Algal turf assemblages cover almost half of all hard substratum, including rubble. Other organisms such as sponges, gorgonians, benthic cyanobactial mats and hydrocorals were usually present but account for relatively little cover. This is the most commonly encountered assemblage covering almost the entire middle section of the fringing reef, including the reefs in front of Kralendijk, the saltpans and on the southern reefs of Klein Bonaire (Figure 6.2).

\section{Coral-Macroalgae}

The coral-macroalgae community is comparable to the lower terrace Macroalgae-CCA reef assemblage in high abundance of macroalgae (also dominated by Dictyota spp. albeit more diverse), crustose coralline algae and low diversity of other taxa. The major difference with the LT cluster is the significantly higher cover of scleractinian coral ( 27\%) (Table 6.2). It appears somewhat contradictory that considerable coral cover coincides with high macroalgal cover, but this is likely a result of the high abundance of hard substratum (virtually no sandy patches). This community is found on the northern reefs of Bonaire, but extending further southward than the 
lower terrace equivalent, therewith including both no-dive reserves (Figure 6.2). This composition is also found at the exposed southern tip of the island.

Table 6.2. Benthic cluster characteristics. Indicator values (indval) and mean percentage cover are shown for the ten main functional benthic groups within each cluster in 2017. Significant indicator values are given in bold, 95\% confidence limits in brackets.

Lower terrace

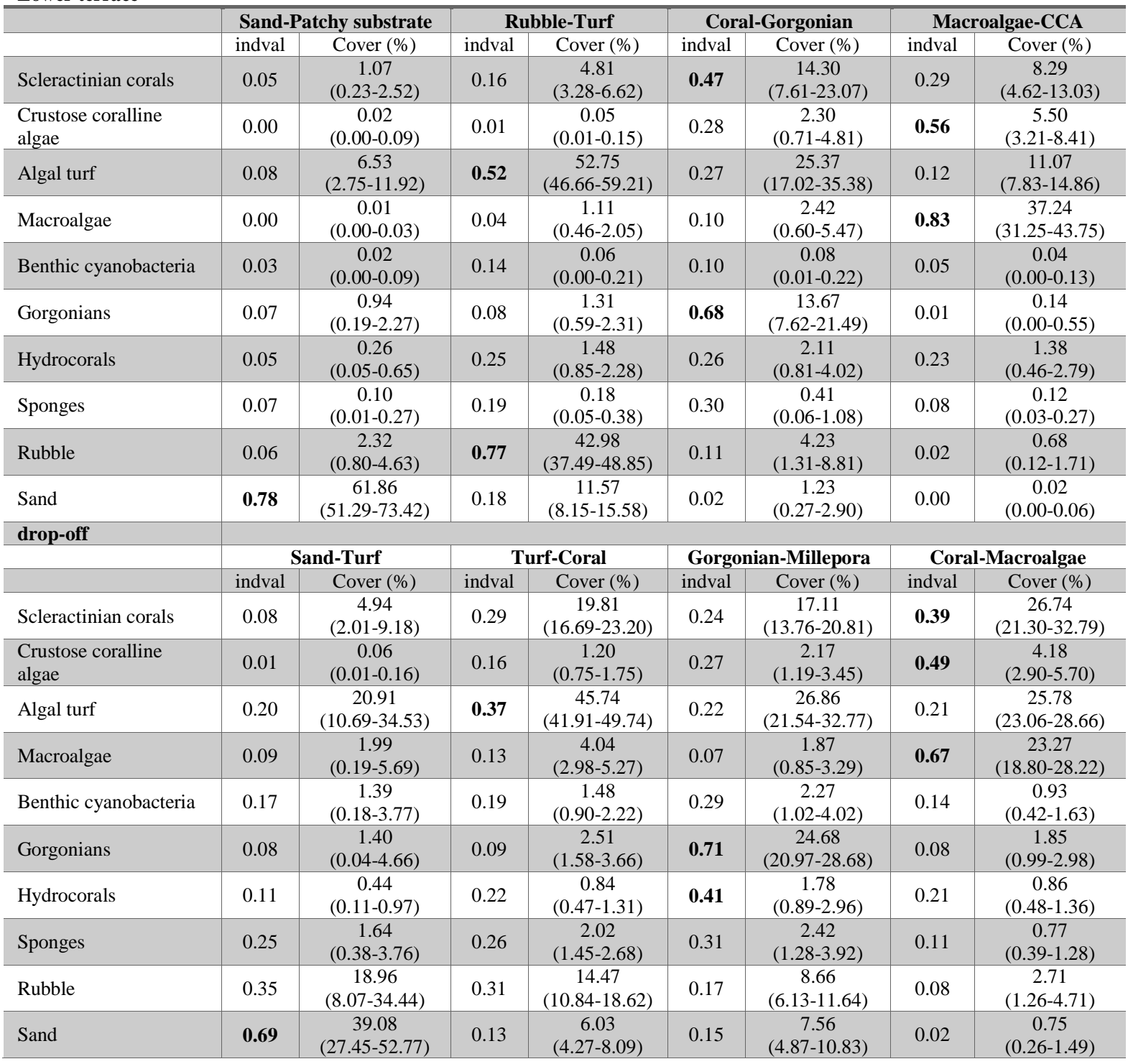




\section{Gorgonian-Millepora}

Among the turf dominated reefs, $17 \%$ of the drop-off reef sites $(n=19)$ were characterized by large stands of gorgonians. These gorgonian forests were mostly found in the southern region of Bonaire (Figure 6.2). Hard coral ( 17\%) and algal turf ( 25\%) cover was substantial at these sites and the relatively high cover of other biotic organisms (hydrocorals, CCA, cyanobacterial mats, sponges and macroalgae) indicates markedly high biological diversity compared to the rest of the fringing reef.

\section{Sand-Turf}

In the drop-off zone, the sand dominated assemblages were less common compared to the lower terrace. It was encountered on the north-eastern side of Klein-Bonaire and at a few sites in the far north and south on the main island (Figure 6.2). Ancillary to sand (39\% cover), these communities were characterized by algal turf covered substratum $(21 \%)$ and rubble (19\%). Hard substratum was relatively abundant compared to the lower terrace Sand-Patchy substrate arrangement. This appears to supports the higher prevalence of other biota with respect to its LT equivalent. Diversity, however, is still marginal compared to the three other drop-off communities (Table 6.2). Coral only accounted, on average, for $\sim 5 \%$ and mainly consisted of foliated coral assemblages dominated by Agaricia spp. (Figure 6.3).

\section{Coral assemblages}

A total of 35 distinct coral species were identified across the 460 studied transects in 2017 , compared to 37 in 2014. Slightly more species were encountered in the drop-off zone $(\mathrm{n}=32)$ compared to the lower terrace zone $(\mathrm{n}=28$ ) (Appendix 6.E). Orbicella spp. (O. annularis, $O$. faveolata and $O$. franksi) were still the dominant corals on the shallow reef of Bonaire, together accounting for $38 \%$ and $48 \%$ of the total coral cover in the lower terrace zone and the drop-off zone, respectively. Apart from these framework-building corals, the coral community in the lower terrace zone is predominately characterized by opportunistic and stess-tolerant species such as P. astreoides, Agaricia agaricites, Pseudodiplora spp., and M. mirabilis. In the dropoff zone, $A$. agaricites was a particularly abundant opportunistic species, while $P$. astreoides and M. mirabilis were less prevalent. Altogether, the seven species described above accounted for over 90\% of the total coral cover in 2017 (Table 6.3 \& Appendix 6.E). Contrastingly, Acropora spp. accounted for less than $1 \%$ of the total coral cover. Although minor variation was observed in the relative contribution of species within the coral community composition in 2014 compared to 2017, the general community structure had not changed (PERMANOVA, $F=0.83$, $p=0.59$ ). Accordingly, the seven prominent coral species remained the dominant components in the coral assemblage over the studies time-period (Appendix 6.E).

Although the drop-off zone was more diverse in terms of species richness (Table 6.3), the general species composition in the observed coral assemblages was usually fairly monospecific compared to the lower terrace zone. Three coral assemblages dominate the drop-off zone, with the Orbicella spp. assemblages being by far the most ubiquitous. The foliated assemblage was characterized by Agaricia spp. with small Eusmilia fastigiata and or Madracis decactis colonies and was generally found in front of Kralendijk and in the southern region of the island. In the 
southern region, as well as to the north of Kralendijk several Madracis mirabilis dominated sites were encountered. Different coral assemblages occurred in the northernmost exposed sites, although here coral cover was generally low. Overall, however, coral cover can be considered fairly good in the drop-off zone. Contrastingly, many lower terrace assemblages were in a poor condition, especially in front and south of Kralendijk. Here, corals had completely disappeared in 10 sites, yet there were abundant Acropora cervicornis stands in the early 1980s (Van Duyl et al. 1985). The lower terrace zone used to be dominated by Acropora spp., but currently such species assemblages (Competitive sensu Darling et al. 2012) have become a scarcity (Figure 6.3). While Orbicella spp. assemblages are still prevalent, many communities are characterized by opportunistic boulder shaped corals such as $P$. astreoides and Pseudodiplora spp. and other resistant species such as Meandrina meandrites and Diploria labyrinthiformis (Figure 6.3: resistant species assemblage). In the lower terrace zone most coral communities could be classified as being in a poor or critical state according to the AGRRA 2018 classification, in particular in front of Kralendijk (Figure 6.3A). Several assemblages considered to be in a relatively good state in terms of cover were found on the reefs north of Kralendijk approximately until the BOPEC, a region that also includes one of the no-dive reserves. With the exception of the northernmost region, AGRRA standards classify most of the drop-off coral communities as relatively good in terms of coral cover (Figure 3B). While this may nowadays be true relative to the wider geographical region, cover across the drop-off zone is also considerably lower than it was the 1980s, see Van Duyl et al. 1985.

Table 6.3. Coral community composition. Relative contribution to the total coral cover of the ten most prominent coral species in the lower terrace zone and drop-off zone in 2017. Mean Shannon-Wiener diversity index (H-index) and species richness indicate the diversity of the coral species assemblage in both zones along $10 \mathrm{~m}$ long benthic transects.

Lower terrace

\begin{tabular}{l|l|l|l|c|}
\hline & Species & $(\%)$ & Species & (\%) \\
\hline Relative contribution & Orbicella annularis & 30.13 & Orbicella annularis & 34.18 \\
\hline to coral total cover & Porites astreoides & 19.16 & Agaricia agaricites & 28.61 \\
\hline & Agaricia agaricites & 14.39 & Orbicella faveolata & 12.16 \\
\hline & Pseudodiploria spp. & 11.31 & Porites astreoides & 7.97 \\
\hline & Madracis mirabilis & 10.22 & Madracis mirabilis & 6.09 \\
\hline & Orbicella faveolata & 7.55 & Montastraea cavernosa & 3.97 \\
\hline & Siderastrea siderea & 2.63 & Madracis decactis & 2.17 \\
\hline & Diploria labyrinthiformis & 2.31 & Siderastrea siderea & 0.92 \\
\hline & Acropora cervicornis & 0.77 & Colpophyllia natans & 0.82 \\
\hline & Meandrina meandrites & 0.38 & Meandrina meandrites & 0.78 \\
\hline H-index & \multicolumn{2}{|c|}{$0.51(0.40-0.64)$} & & 1.23 (1.13-1.33) \\
\hline species richness & $1.97(1.52-2.50)$ & & 6.27 (5.71-6.86) \\
\hline
\end{tabular}




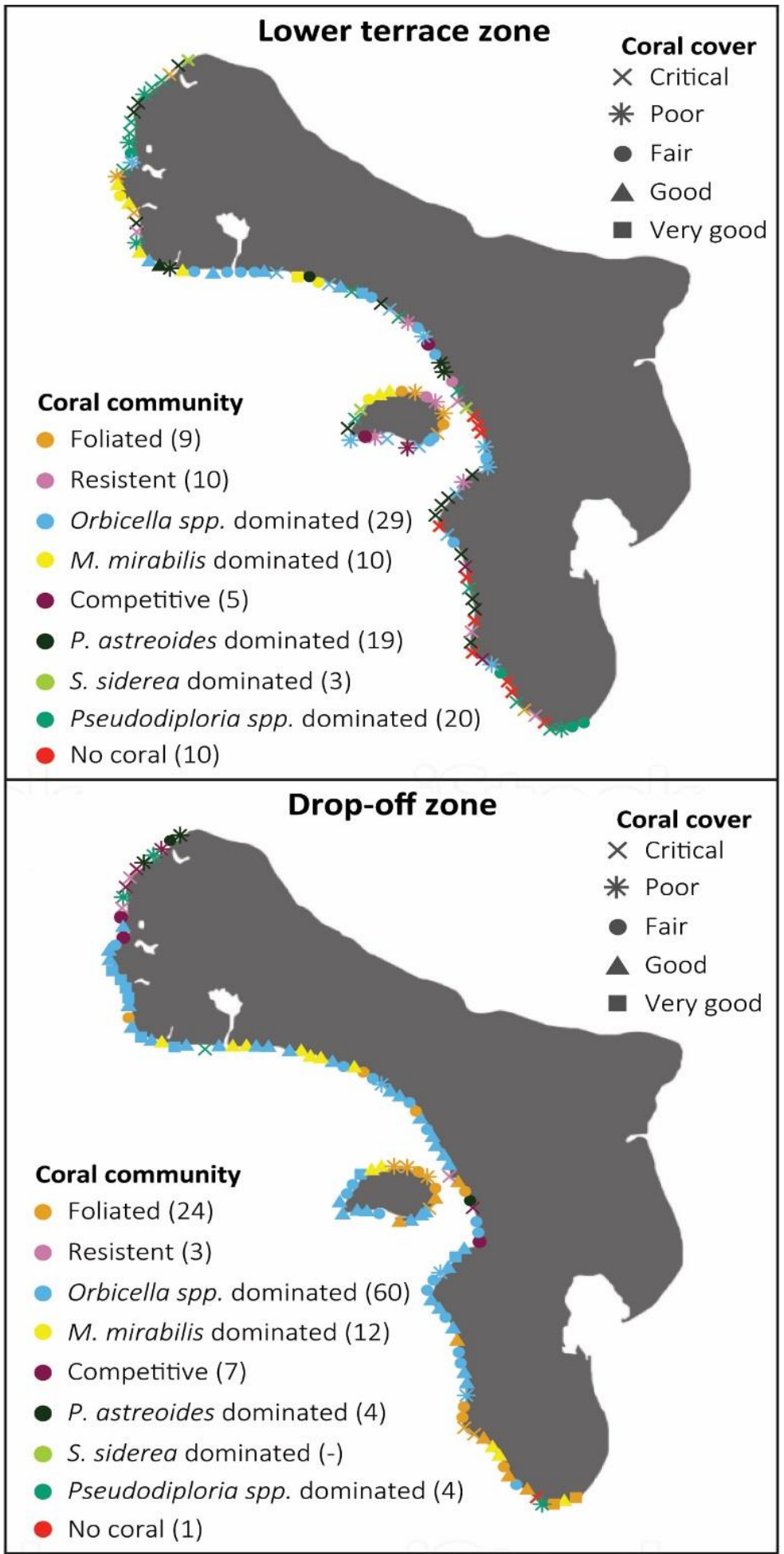


Figure 6.3. Spatial distribution of coral communities based on clustering. Clustering was achieved through single linkage agglomerative clustering based on the scleractinian coral species composition and relative species cover in 2017. Cluster labels were chosen to represent the community composition or dominant species (derived from Indicator species analysis). Symbols indicate the status of the coral community based on the mean percentage coral cover, according to the 2018 AGRRA classification (symbols). Critical: < 5\%; Poor 5\% < 10\%; Fair 10\% < 20\%; Good: 20\% < 40\%; Very good: > 40\%. In all panels, the number of sites in each cluster is given in brackets. Coral species assemblages ( $\boldsymbol{C}$ and $\boldsymbol{D})$ are described in the Coral community section.
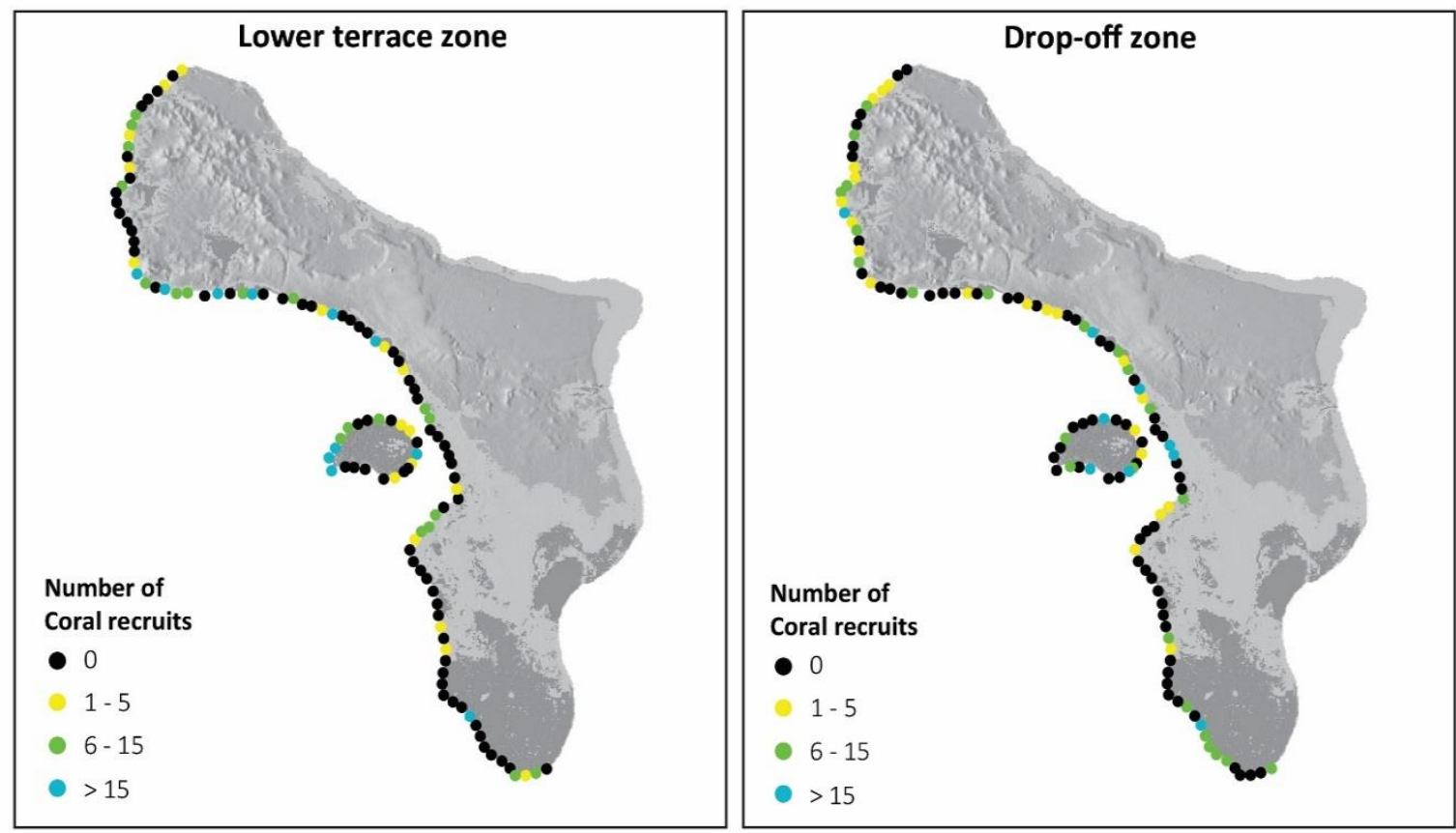

Figure 6.4. Coral recruitment on the shallow reef of Bonaire and Klein Bonaire in 2017. Counts include Scleractinian corals and Milleporidae. Colours represent the number of recruits encountered per square meter at each reef site (zones were pooled).

\section{Coral recruitment}

On average we found 1.46 coral recruits per square meter (95\% CI: 1.00-2.01). Densities were similar in both reef zones, 1.41 recruits $\mathrm{m}^{-2}$ on average in the lower terrace zone and 1.51 recruits $\mathrm{m}^{-2}$ at the drop-off. The majority of the recruits were of the Agariciidae family, followed by recruits belonging to Poritidae and Faviidae (Table 6.4). Five recruits could not be identified. Management policy (i.e. no diving) did not significantly affect recruitment. Several sites directly in front of Kralendijk, for instance, harboured relatively many recruits (Figure 6.4). Coral recruitment was, however, strongly positively correlated to coral cover $\left(F_{(1,228)}=9.12, p<0.01\right)$ and the amount of available hard substratum $\left(F_{(1,228)}=4.67, p=0.03\right)$. Crustose coralline algae cover, known to promote coral settlement, on itself did not correlate significantly to the number 
of recruits, although recruitment appears to be promoted when in combination with high coral $\operatorname{cover}\left(F_{(1,226)}=4.42, p=0.04\right)$.

\begin{tabular}{l|c} 
Family & No. of recruits \\
\hline Agariciidae & 111 \\
\hline Poritidae & 75 \\
\hline Faviidae & 46 \\
\hline Pocilloporidae & 37 \\
\hline Milleporidae & 27 \\
\hline Siderastreidae & 6 \\
\hline Meandrinidae & 6 \\
\hline Caryophylliidae & 3 \\
\hline Unknown & 5 \\
\hline
\end{tabular}

Table 6.4. Number of recorded recruits per coral family in 2017. Recruits were counted in six $25 \mathrm{~cm}^{2}$ quadrats per transect. The number of recorded recruits was pooled for all 115 sites and both zones.

\section{Ecologically significant biota}

In total, 101 Diadema antillarum were counted in 2017, of which only eight were larger or equal to $5 \mathrm{~cm}$ in testes size. The majority of $D$. antillarum were found in the drop-off zone $(\mathrm{n}=75)$ compared to the lower terrace zone $(n=20)$. However, they were generally larger in the lower terrace zone $31.04 \mathrm{~mm}(95 \% \mathrm{CI}: 25.09-37.98)$ test size, than in the drop-off zone, $21.97 \mathrm{~mm}$ (95\% CI: 19.51-24.67). The largest specimens were seen on the reef in front of the harbour of Kralendijk (Appendix 6.F). There was no indication for larger or higher abundance of $D$. antillarum in the no-dive reserves. Trididemnum solidum was encountered under the transect line 29 times. Surprisingly they were almost exclusively found in the drop-off zone north of Kralendijk, including in both no-dive reserves (Appendix 6.F).

\section{General status of the reef fish of Bonaire}

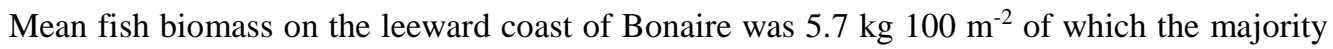
was found in the drop-off zone (Table 6.5). Compared to 2014 there appears to be a slight increase in total fish abundance and biomass (Table 6.5). Analogous to this, an increase in the abundance of commercial fish (groupers and snappers) was observed (Table 6.5), although they are still in critical (83 sites) or poor (26 sites) state in terms of biomass (Figure 6.5C). Similarly, the herbivorous Surgeonfish increased in abundance and biomass and although more Parrotfish (major herbivores) were seen in 2017, their mean biomass did not increase significantly since smaller fish were observed more frequently. The less common, but larger Parrotfish species (Rainbow, Blue and Midnight Parrotfish), were less abundant compared to 2014 as well. Several sites in or directly adjacent to the no-fishing zone harbour a considerable fish biomass relative to the rest of the reefs (Figure 6.5A). Although not consistently, this appears to be the case for herbivorous and commercially important fish guilds as well. Overall mean total fish biomass was higher in the no-fishing areas (7.6 kg $\left.100 \mathrm{~m}^{-2}, 95 \% \mathrm{CI}: 5.7-9.9\right)$ and the no-diving areas (6.5 kg $\left.100 \mathrm{~m}^{-2}, 95 \% \mathrm{CI}: 5.4-7.4\right)$ compared to mean for the rest of the island. Herbivorous fish biomass was particularly high on the reefs north of the Kralendijk and around Klein Bonaire (Figure 6.5B). The most diverse fish communities appear to be found as well (Figure 6.5D). The least diverse fish assemblages were encountered in front of the saltpans in the southern region of the island and between de no-fishing reserves in front of Kralendijk. 
Table 6.5. Fish community characteristics. Overview of total mean fish biomass (gram per 100 $\mathrm{m}^{2}$ ) and the biomass of the major fish guilds in 2014 and 2017. Total biomass is given for both reef zones (230 transects) and the entire shallow reef of Bonaire (460 transects) and based on $4^{\text {th }}$-root transformed transect biomass. Species richness and Shannon-Wiener index (H-index) represent the diversity of the fish community per transect. Total number of fish species is given for each year, as well as the total number of invasive lionfish. 95\% confidence limits are given in brackets. *Not all fish were identified to species level.

2014

2017

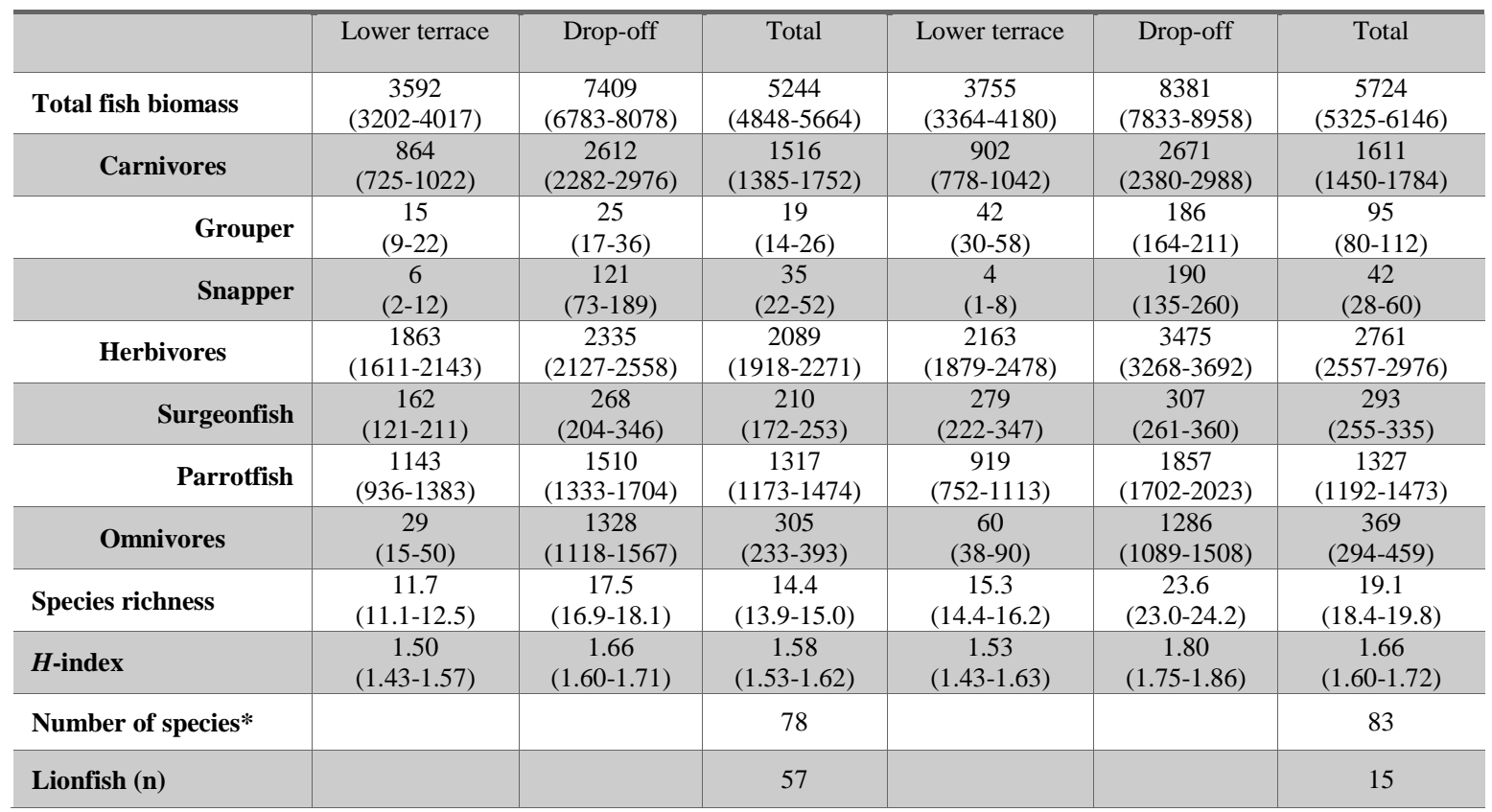

Figure 6.5. Status of the total fish community and the most relevant fish guilds in 2017. Colours represent the status of the total biomass of all fish $(\boldsymbol{A})$, major herbivorous fish families (B) and commercially important fish $(\boldsymbol{C})$ according to the 2018 AGRRA limits at each study site (dots). The number of sites represented by each category are given in brackets. The main fish groups are represented in their respective map. Mean biomass (Mean) across the leeward reef of Bonaire and in the no-fishing zones (NF) is given at the top-right. Fish community diversity (i.e. Shannon-Wiener index) is represented by a continuous colour scale in $\boldsymbol{D}$, with lowest diversity given in black and highest diversity in dark purple. Here, the mean number of fish species is presented in brackets. 

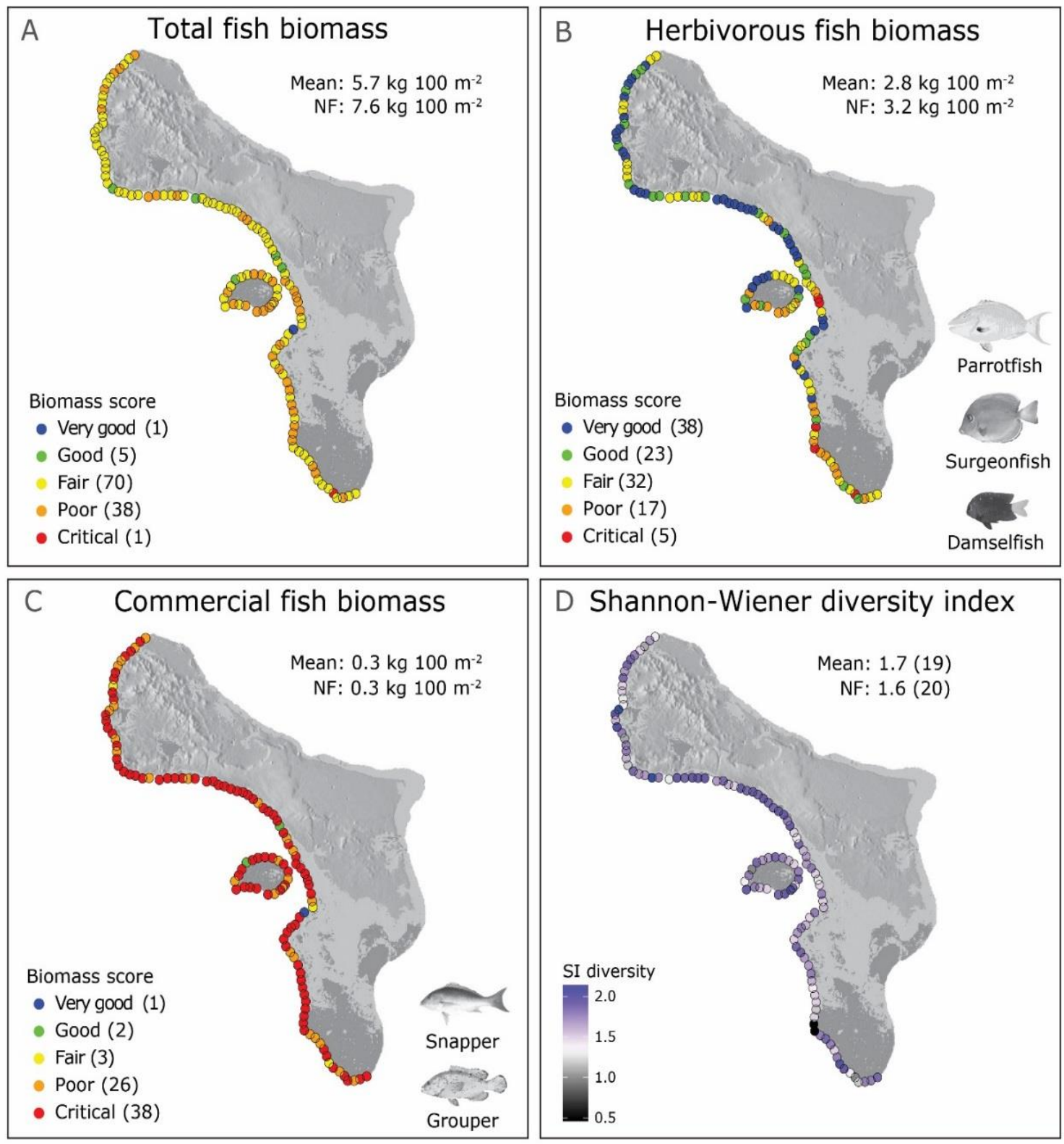
Table 6.6. Species specific contribution to total abundance and biomass in 2017. Total abundance and biomass (gram per $100 \mathrm{~m}^{2}$ ) is given for the ten most prominent fish species (common English names).

Lower terrace

Drop-off

\begin{tabular}{|l|c|c|l|c|c}
\hline \multicolumn{1}{|c|}{ Species } & Count & $\begin{array}{c}\text { Relative } \\
\text { \% }\end{array}$ & \multicolumn{1}{|c}{ Species } & Count & $\begin{array}{c}\text { Relative } \\
\text { \% }\end{array}$ \\
\hline Brown chromis & 27144 & 34.9 & Bicolor damselfish & 40365 & 38.0 \\
\hline Bicolor damselfish & 20316 & 26.1 & Brown chromis & 28423 & 26.8 \\
\hline Blue chromis & 8042 & 10.3 & Blue chromis & 7833 & 7.4 \\
\hline Creole wrasse & 2750 & 3.5 & Queen parrotfish & 2080 & 2.0 \\
\hline Three-spot damselfish & 2038 & 2.6 & Longfin damselfish & 1849 & 1.7 \\
\hline Yellow goatfish & 1881 & 2.4 & Princes parrotfish & 1725 & 1.6 \\
\hline Princes parrotfish & 1685 & 2.2 & Yellow goatfish & 1716 & 1.6 \\
\hline Bluehead wrasse & 1131 & 1.5 & Three-spot damselfish & 1691 & 1.6 \\
\hline Longfin damselfish & 1102 & 1.4 & Blue tang & 1644 & 1.5 \\
\hline Stoplight parrotfish & 945 & 1.2 & Stoplight parrotfish & 1485 & 1.4 \\
\hline \multicolumn{1}{|c|}{ Species } & Biomass & Relative & Species & Biomass & Relative \\
\hline Stoplight parrotfish & 506 & 16.6 & Stoplight parrotfish & 356 & 11.1 \\
\hline Schoolmaster snapper & 202 & 6.6 & Bicolor damselfish & 303 & 9.4 \\
\hline Brown chromis & 188 & 6.2 & Queen parrotfish & 236 & 7.3 \\
\hline Queen parrotfish & 145 & 4.8 & Brown chromis & 198 & 6.2 \\
\hline Black durgon & 137 & 4.5 & Smallmouth grunt & 181 & 5.6 \\
\hline Bicolor damselfish & 134 & 4.4 & Blue tang & 146 & 4.5 \\
\hline Princes parrotfish & 131 & 4.3 & Princes parrotfish & 132 & 4.1 \\
\hline Smallmouth grunt & 122 & 4.0 & Squirrelfish & 113 & 3.5 \\
\hline Yellow goatfish & 113 & 3.7 & Schoolmaster snapper & 108 & 3.4 \\
\hline Yellowtail snapper & 110 & 3.6 & Black durgon & 102 & 3.2 \\
\hline
\end{tabular}

\section{Fish community}

The Bicolored Damselfish was by far the most commonly observed species (39\% of all encountered fish) on the shallow reef of Bonaire in 2017, followed by the Brown and Blue Chromis (27\% and 7.4\%, respectively), the Queen Parrotfish (2\%), the Longfin Damselfish (1.7\%), the Princes Parrotfish (1.6\%) and the Yellow Goatfish (1.6\%). In 2014, the same species dominated the fish community on the leeward side of the island, albeit in a different order of abundance (Table 6.6). It is noteworthy that only half as many Bicolor Damselfish were spotted compared to 2017. In 2017 more groupers where encountered, and Graysby and Coneys were the most commonly spotted grouper species. While species such as the Rock Hind and the Tiger grouper were still seen in 2014, these were pretty much absent in 2017. An increase in abundance of the overharvested Graysby (small grouper) and Giant Barracuda was observed possibly suggesting some degree of recovery. Damselfish, Parrotfish and Wrasses were the dominant families. The Stoplight Parrotfish was the most dominant species in terms of biomass, followed by the Bicolored Damselfish, Queen parrotfish, Brown Chromis and Smallmouth Grunt. Again, the species that contribute most evidently to the total fish biomass were very similar among both years (Table 6.6), although it appears that the Schoolmaster Snappers were 
smaller in size in 2017. While it is apparent which species dominate the fish stocks on Bonaire's leeward reefs, caution should be exercised when comparing differences in biomass among years on a species level. Since biomass is based on size estimates by different surveyors, some degree of surveyor bias cannot fully be excluded. In either years, no sharks or rays were encountered within the surveys. Overall, substantially less and smaller invasive lionfish (Pterois volitans) were seen in 2017 compared to 2014.

\section{Discussion}

The elaborate assessment of the condition in which the shallow leeward reefs of Bonaire currently exist, evidently indicates signs of global and local negative human impact. This is certainly not unexpected and confirms previous recordings implying that the fringing reef around Bonaire has not escaped the ecological degradation as seen throughout the wider Caribbean following continuously exerted anthropogenic stress (Bak et al. 2005; Sommer et al. 2011; De Bakker et al. 2017). The conducted surveys, however, also clearly illustrate that variations in local conditions (both natural and anthropogenic) can have a significant effect on reef community configuration, resulting in considerable variability on a fine spatial scale. Historically, benthic reef community composition were predominantly shaped by variations in natural conditions such as exposition and geomophology (Van Duyl 1985). On present-day reef, however, the arrangement of reef assemblages appears to be much more an outcome of the severity of anthropogenic induced stress.

\section{Status and trends in benthic and fish communities on the shallow reef of Bonaire}

The degree of stress that is locally and globally exerted upon the reef system of Bonaire has, in combination with aspects of the local natural environment, resulted in the formation of benthic communities that show limited resemblance to configurations as described for the same reefs several decades ago (Scatterday 1974; Bak 1975; Van Duyl 1985; Bak and Nieuwland 1995; De Bakker et al. 2017). While a distinct zonation (Bak 1977) still exists across the reef, we propose a novel description of the reef communities within the lower terrace and drop-off zones. Where reef communities were historically described by their cover and assemblage of scleractinian corals they are presently characterized by the abundance of sand, rubble and algal turf covered dead coral substratum. Our findings provide discernible support for the assumption that turf communities, dominated by small filamentous algae, have become the most prominent biotic functional group on many coral reefs (McCook 2001; Gorgula and Connell 2004). Since herbivorous pressure has declined ubiquitously due the overharvesting of reef fish and due to the virtual absence of $D$. antillarum since the mass die-off in 1983, the abundance of algal turf is by large determined by the availability of substratum and terrestrial impact (i.e. sedimentation and eutrophication) (Vermeij et al. 2010; Smith et al. 2016). The relatively low abundance of $\mathrm{BCM}$ is somewhat surprising considering the high cover found at several reef sites of Curaçao and Bonaire in 2013 (De Bakker et al. 2017). Variability in the environmental conditions of the studied zones, reef flat (this study) versus the reef slope (De Bakker et al. 2017) may explain some of the observed variation. Shifts in their abundance are, however, more likely related to 
their specific opportunistic life history traits. They appear to be able to flourish rapidly when environmental conditions are favourable, but also largely recede again when conditions change.

Despite the overall prominent abundance of algal turfs, divergent compositions in other reef biota allowed classification of eight distinct shallow reef communities. The distribution of these communities along the leeward coast of Bonaire appears to reflect, at least in part, the intensity of local human impact (Figure 6.2). More diverse communities, where historically prevalent organisms (scleractinian corals, hydrocorals and gorgonians) still occur in moderate to high abundance, were encountered to the north and south of Kralendijk and away from direct urban impact. Contrastingly, the reefs in front of Kralendijk are generally in a poor to critical condition (Figure 6.2 \& Figure 6.3). This is especially evident on the lower terrace zone, which is impacted more directly by coastal activities than the drop-off zone. Here, solid substratum on which organisms could settle is hardly available and the majority of the bottom is currently covered by sand. Low cover of corals and high abundance of unfavourable taxa such as turf and BCMs currently characterize these reefs. In regions where human coastal activity is less pronounced, some configurations, like the macroalgal stands in the northernmost region of Bonaire, appear to still largely be driven by local hydrological and geomorphic features (Leigh et al. 1987; Renken et al. 2010; Roff et al. 2015). The formation of these reef communities along a gradient of human impact may display distinct reef configurations found on reefs with comparable geomorphological characterization throughout the wider Caribbean.

Although at present, corals are often no longer the dominant reef constituent, they could locally still reach considerable cover, most notably in the drop-off zone. On the reefs of Bonaire Orbicella spp. are still the dominant corals with regards to cover, however many coral communities have shifted to a dominance of more opportunistic corals such as P. astreoides, A. agaricites, Madracis spp. and (Pseudo)diploria spp. (Figure 6.3). Such a trend has previously been described on Caribbean reefs (Knowlton 2001; Green et al. 2008; De Bakker et al. 2016b) and may mark the prevalence of stress-tolerant coral species as the low-abundance contribution of scleractinian corals to current reef communities. The latter appears to be supported by the strong representation of Agariciidae and Poritidae among the observed juvenile coral settlers on Bonaire and other reefs in the Caribbean (Mumby et al. 2007; Arnold et al. 2010; Vermeij et al. 2011; this study). Coral recruits were observed at sites across the entire reef stretch, but overall at relatively low densities, especially compared to past abundances (Bak and Engel 1979; Vermeij et al. 2011). Although recruitment across the shallow reef of Bonaire appears erratic (Figure 6.4) successful settlement appeared to be positively linked to high coral cover and the presence of available substratum to settle on (e.g CCA). That coral recruitment is impeded by considerable algal turf cover and a limited adult source population is in compliance with previous findings (Vermeij and Sandin 2008; Arnold et al. 2010). As a methodological consideration, it should also be pointed out that identifying coral recruits based on size, as performed in study, can sometimes be misleading since size is not linearly related to the age of a colony (Vermeij et al. 2011). 
Reef fish communities, like their benthic counterpart, have clearly been impacted through anthropogenic pressure, marked for instance by the almost near absence of apex predators (Sandin et al. 2008a; this study). Variation in total biomass across the entire shallow reef was limited and ranged between poor and fair at almost all sites. The designation of no-fishing zones does appear to lead to slightly higher abundance and biomass of fish in general, including herbivorous fish. Overall, herbivorous fish can be considered relatively abundant on the shallow reef of Bonaire, especially compared to the wider Caribbean region (e.g. Jackson et al. 2014). The biomass of groupers and snappers, fish that are important for commercial fisheries in Bonaire (De Graaff et al. 2016), can at present, however, be considered critical across the shallow reef according to the Healthy Reef Initiative standards, regardless of benthic community composition or implemented conservation legislation. Abundance of the invasive lionfish $(P$. volitans) has declined substantially between 2014 and 2017. This may be an effect of considerable removal efforts on the island of Bonaire since the introduction the species in 2009 (e.g. De León et al. 2013).

\section{Towards stabilization of decline on the shallow reef of Bonaire}

Aforementioned findings clearly underline that anthropogenic pressure has strongly affected the shallow reef of Bonaire over the past decades. Yet, the absence of clear variation in various facets, including cover of corals, the composition of coral and fish assemblages and fish biomass between 2014 and 2017, tentatively insinuates that stretches of shallow reef may at present be adapting to the different levels of disturbance along the coast and display some degree of stabilization in ecological decline. Findings presented in De Bakker et al. (2016b), following coral cover between 1973 and 2013 in permanent quadrats (Karpata, Bonaire and Curaçao), suggest that the continuous decline in coral cover at $10 \mathrm{~m}$ depth has already become less obvious since 2009. Furthermore, by comparing our findings to dedicated surveys conducted by Sommer et al. (2011) on the leeward reefs of Bonaire in 2008 and 2009 (coral cover: $9.47 \pm 1.7,19.70 \pm$ 1.90 , at $5 \mathrm{~m}$ and $10 \mathrm{~m}$ depth respectively), it could be argued that a stabilizing trend on the shallow reef has indeed been occurring already for the past decade, in particular the drop-off zone. Several aspects of the fish assemblage, including an increase in biomass of groupers and snappers and a decline in the number of invasive lionfish, may even support postulations that hint to careful recovery (Sandin et al. 2008a; De Graaf et al. 2016; Steneck et al. 2013; this study).

Whether the apparent stabilization of decline on the shallow reef of Bonaire is indeed occurring and whether it exemplifies a positive effect of local management (including increasingly more sewage treatment since 2015, reduced fishing pressure, lionfish hunting), adaptation, an absence of large-scale exerted stress (i.e. region-wide mass bleaching) or perhaps a combination of these factors can hardly be ascertained based on solely on the data presented here. Yet, these questions are essential in terms of the current vulnerability and resilience of these reefs. It is worth noting that the last mass coral bleaching around Bonaire occurred in 2010 and since then the few passing tropical storms have only caused localized disturbance (Eakin et al. 2010). It thus remains uncertain how these reefs will respond if exposed to longer episodes of thermal stress or other major disturbances in the near future. Furthermore, it seems that several other aspects 
of reef communities, including the cover of fast growing macroalgae and BCMs are still less stable and less predictable (De Bakker et al. 2017 and this study). Hence, it remains to be elucidated whether the current configurations on the reef of Bonaire will indeed stabilize in their current composition and condition and if so, to what extent they are still able to supply specific ecosystem services.

In conclusion, the conducted surveys indicate that large parts of shallow reef on Bonaire are in a poor state with respect to historical baselines. More importantly, however, it appears that certain stretches are currently still in a considerably better condition (i.e. high coral cover, in particular Orbicella spp., low algal abundance, configurations with diverse benthos and fish taxa) than most reefs in the wider Caribbean region. While this has already been reported (Gardner et al. 2003; Newman et al. 2006; Jackson et al. 2014), it seems to confirm that when local conditions are favourable (i.e. limited human impact), coral reefs have the capacity to better cope with global stressors such as climate change. Investigating the conditions that currently support these good reefs should be the basis for adequate management. At present, it seems that the current management practices implemented on Bonaire already have positive localized impact, both on benthos and fish communities. The effects, however, remain largely restricted to the protected areas. Nonetheless, the continuing efforts of Bonaire to expand the sewage system will certainly aid in restoring reef reselience and should be applauded. Continued and extended implementation of adequate legislation is of paramount importance to prevent further degradation and stimulate stabilization and recovery on these reefs. We furthermore acknowledge that the focus of this study lies only on the shallow region of the reef. While this is the region that is most directly impacted by terrestrial disturbance, the status of the reef in its entirety should be studied since communities on the reef slope of Bonaire are shown to be affected by human activities as well (De Bakker et al. 2016b; De Bakker et al. 2017).

The observed indications for stabilization of ecological decline are promising, but most of the reefs along Bonaire are nowadays of insufficient quality to meet ecosystem services that were provided by these reefs in the past (fisheries, capacity to track sea level rise, highly attractive reefs for snorkel and dive tourism). Furthermore, while potential ecological stabilization seems supported by previous surveys, the time period covered in this study is to date still very short to elaborate too much on temporal trends. A longer study period is needed to elucidate whether this stabilizing trend will indeed exists and may continue or whether it has simply become difficult to distinguish a percentage decline over an already low cover over a short period. For this reason, we strongly encourage the continuation of this research and reef surveys on coral reefs worldwide. 


\section{Acknowledgement}

We would like extend our gratitude to Caren Eckrich for her support in the field. We also want to acknowledge the invaluable logistical assistance of the STINAPA Bonaire National Parks Foundation. The data presented here are the initial result of a multi-year program initiated in 2011 by dr. EH Meesters and IJM Van Beek with the aim to elaborately monitor the status of Bonaire coral reefs on an extensive spatial and temporal scale and guide the implementation of adequate management efforts. This study has been funded by the Ministry of Economic Affairs for the purposes of the Policy Supporting Research Theme 'Analyse fotomateriaal koraalrif/fase 1 ' (Project No. BO-11- 019.02-038). The Royal Netherlands Institute for Sea Research provided funding of expenses and in-kind support. 


\section{Appendix 6.A}

Percentage cover of the main benthic categories per transect. Site numbers correspond to the numbers given in Figure 6.2. At each site four transects were surveyed, two in each zone (marked A and B). The implemented conservation practice at a site is indicated in the column 'Area'. The overview table can be accessed through the following link.

https://doi.org/10.18174/472452

\section{Appendix 6.B}

Results of mantel test to define the optimal number of Ward clusters (k) based on Pearson's correlation. Optimal number of clusters is indicated by the red dot for the lower terrace (left panel) and the drop-off zone (right panel).
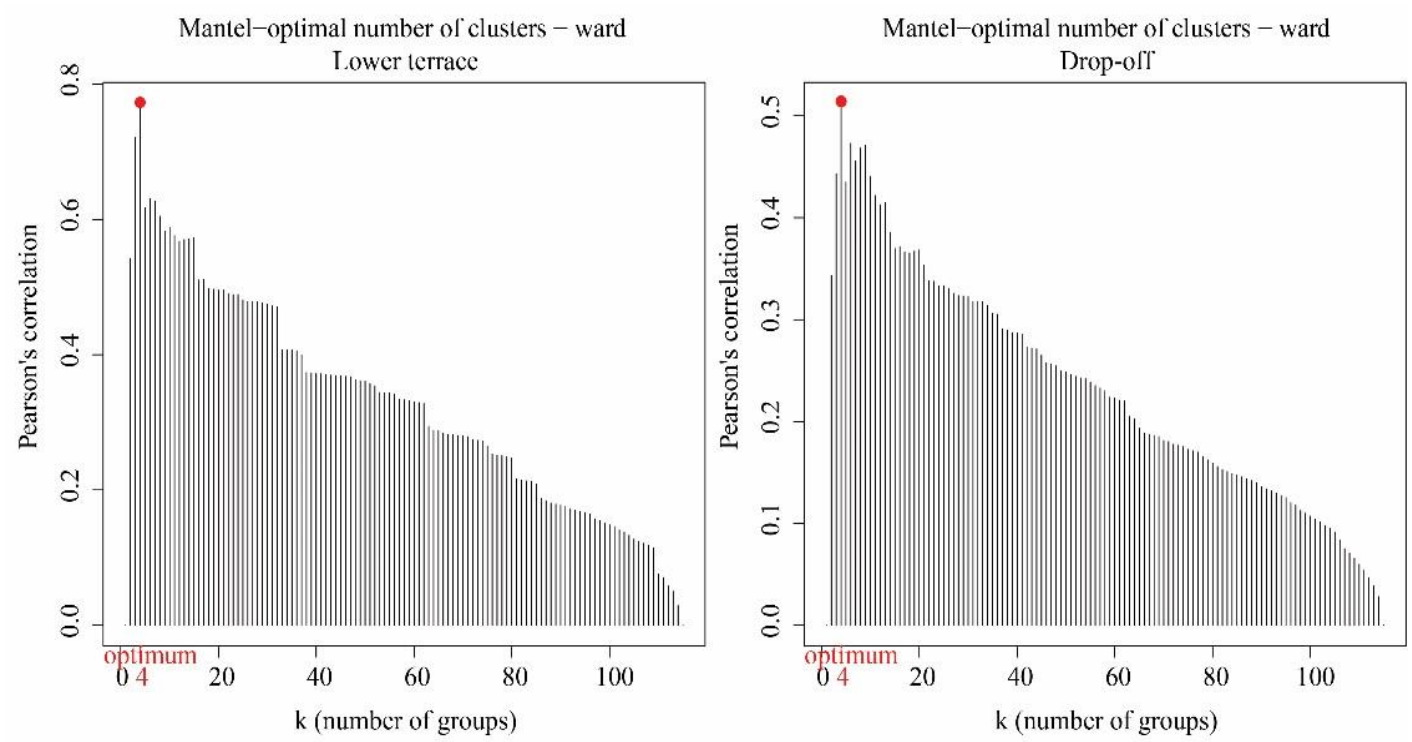

\section{Appendix 6.C}

Overview of the constants ( $a$ and $b$ ) used for the length to biomass conversion for the different fish species. Applied constants are conform the 2018 Atlantic and Gulf Rapid Reef Assessment (AGRRA) methodology. The overview table can be accessed through the following link.

https://doi.org/10.18174/472452

\section{Appendix 6.D}

Mean cover per benthic group in 2017. Dots indicate each of the 115 sites on the leeward side of Bonaire and around Klein Bonaire. Colour ranges indicate the mean percentage cover (two transects) for the nine most dominant benthic components. Colour scales vary for each individual panel. Left panels represent the lower terrace zone, right panels the drop-off zone. 


\section{Chapter VI}

Lower terrace
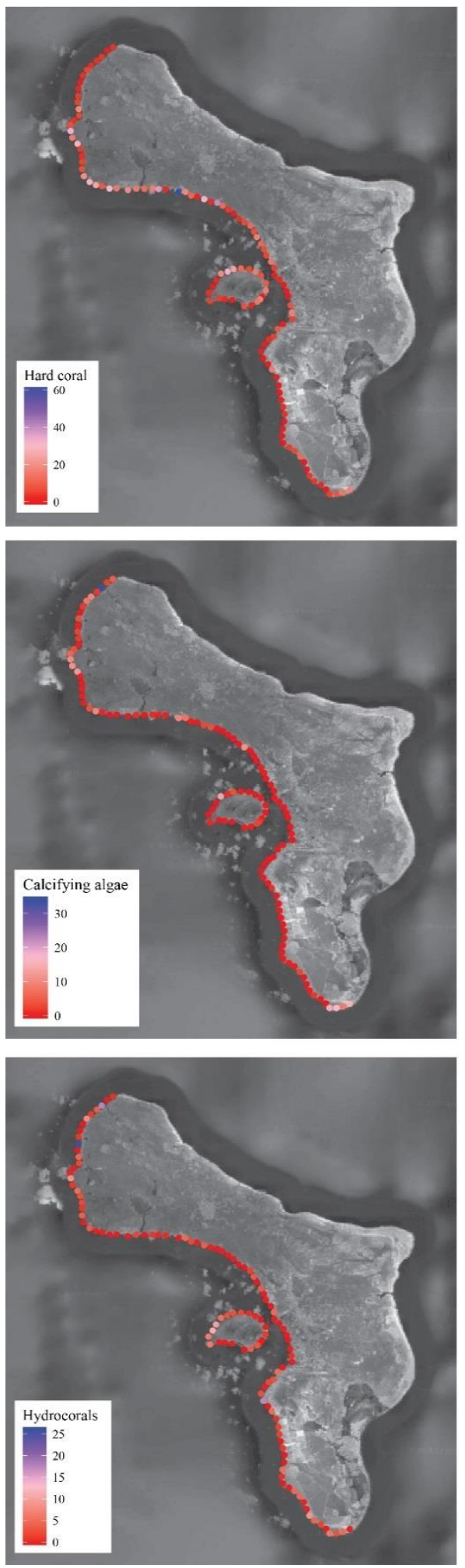
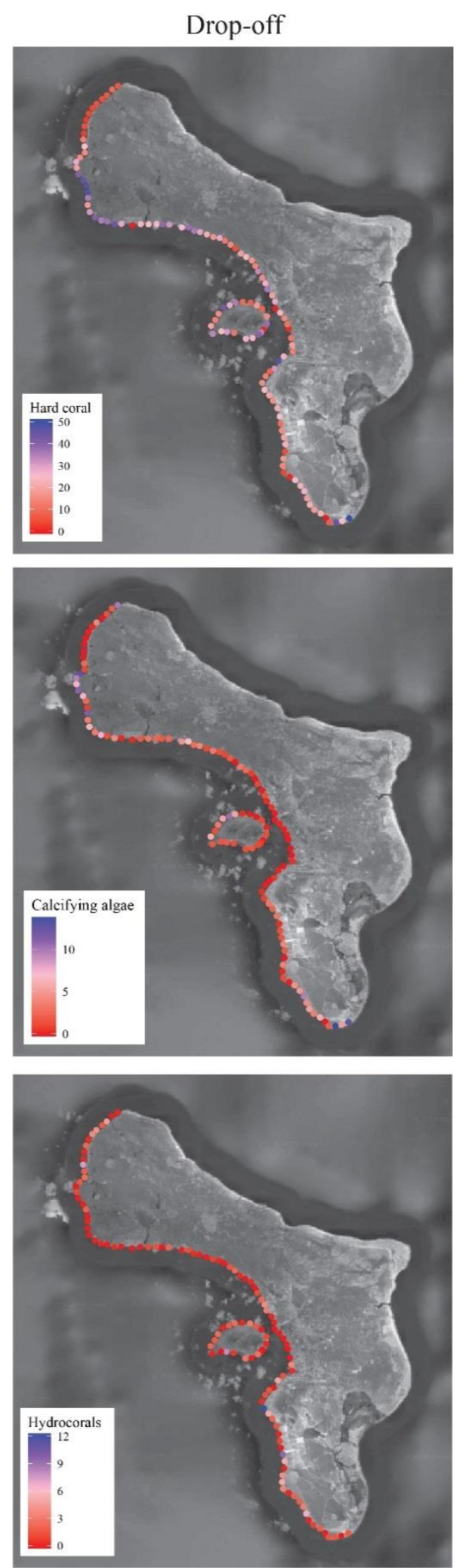
Towards stabilization of the decline on the shallow reefs of Bonaire

\section{Lower terrace}
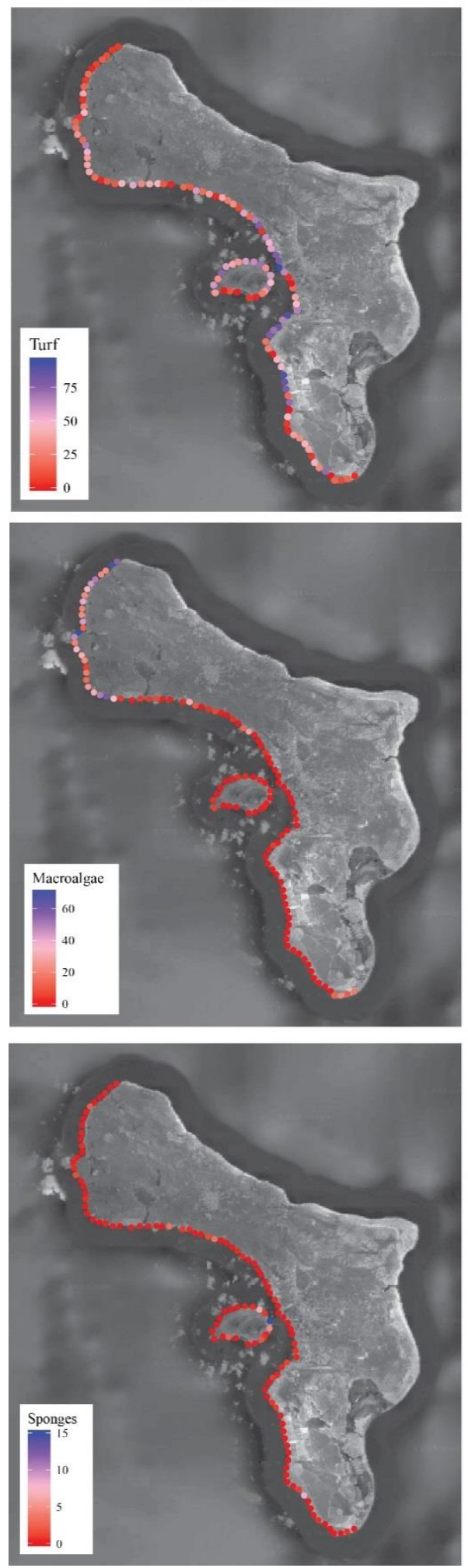

Drop-off
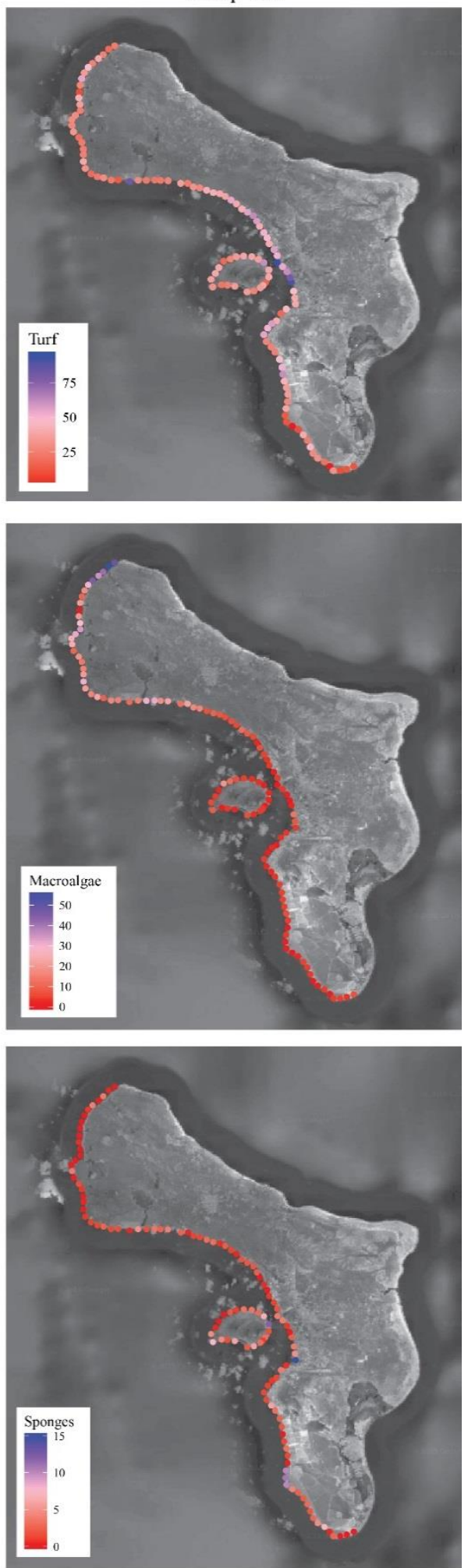


\section{Chapter VI}

Lower terrace
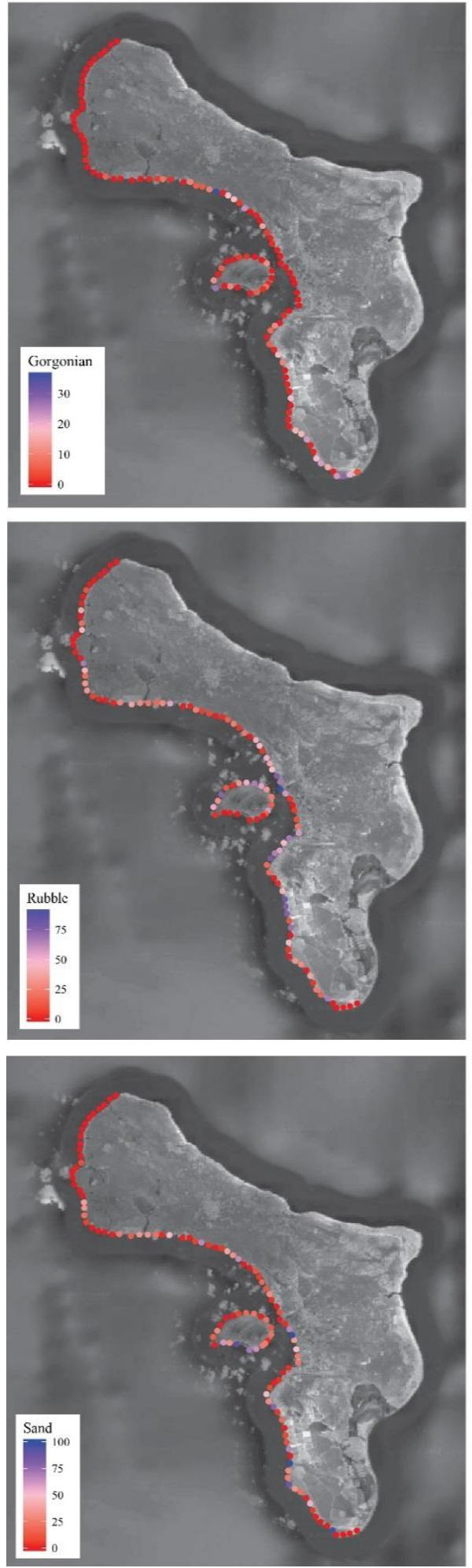

Drop-off
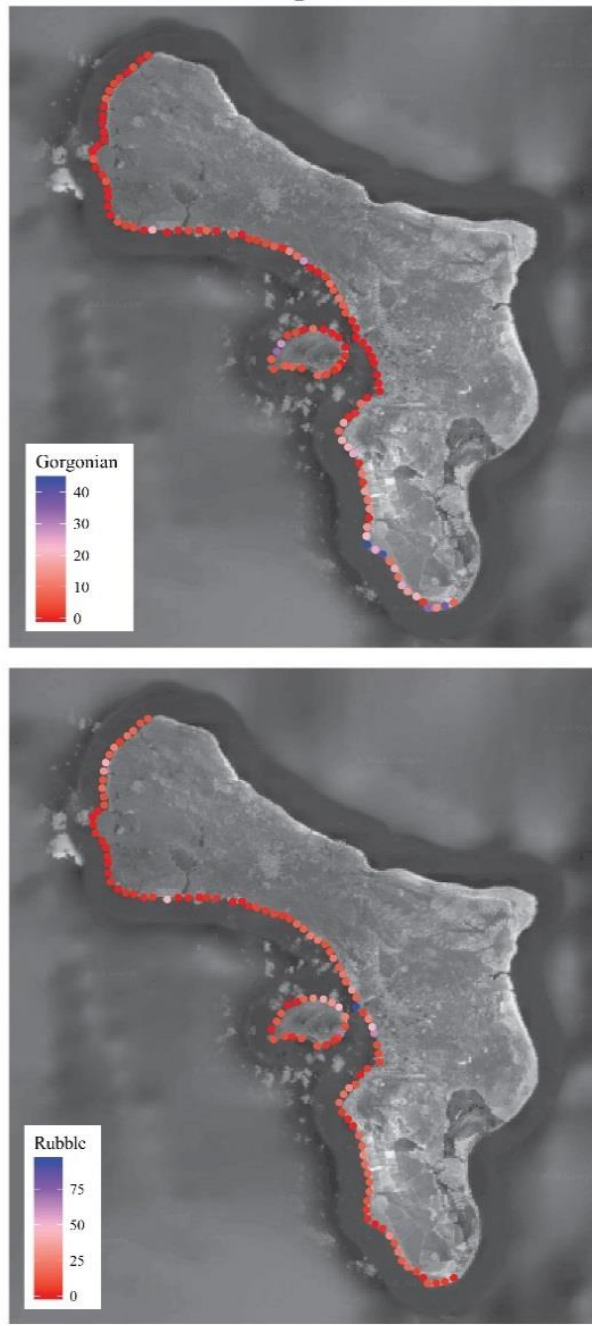

6

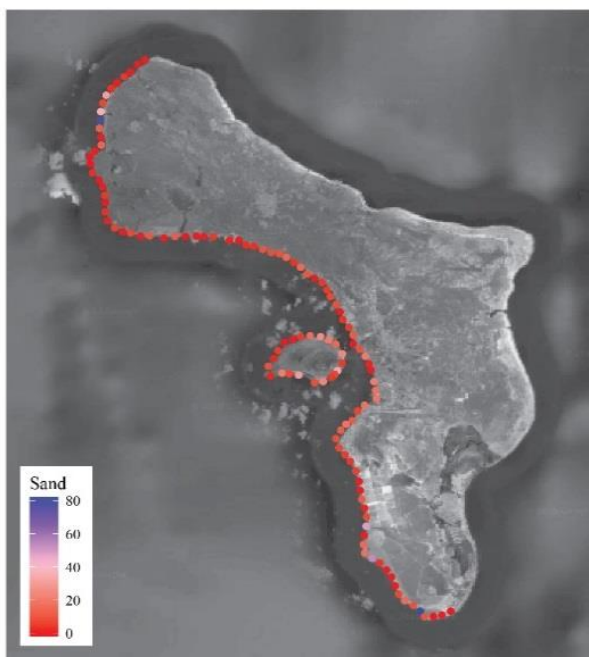




\section{Appendix 6.E}

Percentage cover per scleractinian coral species at each $10 \mathrm{~m}$ benthic transect. Site numbers correspond to the numbers given in Figure 6.2. At each site four transects were surveyed, two in each zone (marked A and B). The overview table can be accessed through the following link.

https://doi.org/10.18174/472452

\section{Appendix 6.F}

Overview of the A) Diadema antillarum and B) Trididemnum solidum encountered in the transects. (n): number of urchins counted within the $20 \mathrm{~m}^{-2}$ transect, mean test size (mm) is given per site standard deviation is given in brackets. Cover refers to the total area $(\mathrm{cm})$ covered by $T$. solidum underneath the $10 \mathrm{~m}$ benthic transect line.

\section{A) Diadema antillarum}

\begin{tabular}{|c|c|c|c|c|}
\hline Site & Zone & Area & $\mathbf{n}$ & $\begin{array}{c}\text { Test size } \\
\text { (mm) }\end{array}$ \\
\hline 3 & Lower-terrace & Marine Park & 1 & 30 \\
\hline 11 & Lower-terrace & Marine Park & 1 & 40 \\
\hline 18 & Lower-terrace & Marine Park & 1 & 20 \\
\hline 20 & Lower-terrace & Marine Park & 1 & 25 \\
\hline 26 & Lower-terrace & Marine Park & 1 & 20 \\
\hline 27 & Lower-terrace & Marine Park & 1 & 40 \\
\hline 34 & Lower-terrace & No Fishing & 1 & 70 \\
\hline 35 & Lower-terrace & No Fishing & 1 & 80 \\
\hline 40 & Lower-terrace & No Fishing & 1 & 20 \\
\hline 53 & Lower-terrace & Marine Park & 1 & 20 \\
\hline 57 & Lower-terrace & Marine Park & 1 & 30 \\
\hline 66 & Lower-terrace & Marine Park & 1 & 35 \\
\hline 93 & Lower-terrace & Marine Park & 1 & 35 \\
\hline 96 & Lower-terrace & Marine Park & 1 & 10 \\
\hline 108 & Lower-terrace & Marine Park & 3 & $33(8)$ \\
\hline 109 & Lower-terrace & Marine Park & 1 & 25 \\
\hline 113 & Lower-terrace & Marine Park & 1 & 40 \\
\hline 115 & Lower-terrace & Marine Park & 1 & 30 \\
\hline 6 & Drop-off & Marine Park & 2 & $18(11)$ \\
\hline 7 & Drop-off & Marine Park & 1 & 20 \\
\hline 10 & Drop-off & Marine Park & 1 & 15 \\
\hline 15 & Drop-off & Marine Park & 1 & 40 \\
\hline 18 & Drop-off & Marine Park & 2 & $18(11)$ \\
\hline 19 & Drop-off & Marine Park & 2 & $28(4)$ \\
\hline 22 & Drop-off & Marine Park & 1 & 40 \\
\hline 25 & Drop-off & Marine Park & 3 & $27(25)$ \\
\hline 26 & Drop-off & Marine Park & 6 & $18(4)$ \\
\hline 27 & Drop-off & Marine Park & 1 & 10 \\
\hline 28 & Drop-off & Marine Park & 1 & 20 \\
\hline 30 & Drop-off & Marine Park & 2 & $18(4)$ \\
\hline 31 & Drop-off & Marine Park & 2 & 30 \\
\hline 32 & Drop-off & Marine Park & 1 & 20 \\
\hline
\end{tabular}




\begin{tabular}{|c|c|c|c|c|}
\hline 34 & Drop-off & No Fishing & 1 & 30 \\
\hline 35 & Drop-off & No Fishing & 2 & $60(14)$ \\
\hline 36 & Drop-off & Marine Park & 1 & 40 \\
\hline 42 & Drop-off & No Fishing & 2 & $23(11)$ \\
\hline 46 & Drop-off & Marine Park & 3 & $18(10)$ \\
\hline 50 & Drop-off & Marine Park & 3 & $27(6)$ \\
\hline 51 & Drop-off & Marine Park & 2 & $30(7)$ \\
\hline 52 & Drop-off & Marine Park & 1 & 20 \\
\hline 55 & Drop-off & Marine Park & 1 & 10 \\
\hline 56 & Drop-off & Marine Park & 1 & 20 \\
\hline 57 & Drop-off & Marine Park & 1 & 20 \\
\hline 58 & Drop-off & Marine Park & 1 & 10 \\
\hline 59 & Drop-off & Marine Park & 2 & $15(7)$ \\
\hline 61 & Drop-off & No Diving & 3 & $18(8)$ \\
\hline 67 & Drop-off & Marine Park & 1 & 25 \\
\hline 68 & Drop-off & Marine Park & 1 & 20 \\
\hline 73 & Drop-off & Marine Park & 1 & 30 \\
\hline 78 & Drop-off & No Diving & 2 & $40(14)$ \\
\hline 90 & Drop-off & Marine Park & 1 & 30 \\
\hline 95 & Drop-off & Marine Park & 2 & $25(7)$ \\
\hline 97 & Drop-off & Marine Park & 1 & 50 \\
\hline 101 & Drop-off & Marine Park & 1 & 25 \\
\hline 104 & Drop-off & Marine Park & 1 & 25 \\
\hline 105 & Drop-off & Marine Park & 1 & 10 \\
\hline 106 & Drop-off & Marine Park & 1 & 10 \\
\hline 108 & Drop-off & Marine Park & 6 & $17(4)$ \\
\hline 109 & Drop-off & Marine Park & 1 & 50 \\
\hline 110 & Drop-off & Marine Park & 1 & 35 \\
\hline 111 & Drop-off & Marine Park & 6 & $18(8)$ \\
\hline 112 & Drop-off & Marine Park & 3 & $28(13)$ \\
\hline 114 & Drop-off & Marine Park & 1 & 30 \\
\hline
\end{tabular}


B) Trididemnum solidum

\begin{tabular}{c|c} 
Site & $\begin{array}{c}\text { Cover } \\
\text { (cm) }\end{array}$ \\
\hline $\mathbf{3 1}$ & 28 \\
\hline $\mathbf{5 1}$ & 25 \\
\hline $\mathbf{5 2}$ & 15 \\
\hline $\mathbf{5 3}$ & 37 \\
\hline $\mathbf{5 4}$ & 4 \\
\hline $\mathbf{5 5}$ & 2 \\
\hline $\mathbf{5 7}$ & 14 \\
\hline $\mathbf{5 8}$ & 31 \\
\hline $\mathbf{6 1}$ & 16 \\
\hline $\mathbf{6 2}$ & 2 \\
\hline $\mathbf{6 3}$ & 3 \\
\hline $\mathbf{6 4}$ & 16 \\
\hline $\mathbf{7 1}$ & 4 \\
\hline $\mathbf{7 2}$ & 7 \\
\hline $\mathbf{7 7}$ & 10 \\
\hline $\mathbf{8 0}$ & 11 \\
\hline $\mathbf{8 1}$ & 16 \\
\hline $\mathbf{8 7}$ & 48 \\
\hline $\mathbf{9 0}$ & 27 \\
\hline $\mathbf{9 3}$ & 17 \\
\hline $\mathbf{1 0 6}$ & 4 \\
\hline
\end{tabular}

\section{Appendix 6.G}

Abundance (per $100 \mathrm{~m}^{2}$ ) and biomass (gram per $100 \mathrm{~m}^{2}$ ) per fish species at each site. Site numbers correspond to the numbers given in Figure 6.2. At each site four transects were surveyed, two in each zone (marked A and B). The overview table can be accessed through the following link.

https://doi.org/10.18174/472452 
Chapter VI 


\section{CHAPTER VII \\ General discussion: is there hope for Caribbean coral reefs?}

Didier M de Bakker

There lies little novelty in the claim that coral reef ecosystems are experiencing large-scale ecological degradation due to increased anthropogenic impact. Yet, the implications that the changing marine environment has for reef ecosystem functioning and configurations of reef biota are complex and often erratic. The aim of this thesis was to contribute to our holistic understanding of how these ecosystems respond to the various facets of explosive human population growth.

\section{Trends in benthic community configurations}

During this project, I documented changes in benthic coral reef assemblages over recent decades. What the studied time-series and surveys immediately elucidate is that corals are indeed the most obvious casualty of the changing reef environment. Mean coral cover on the reef slopes (10 - $40 \mathrm{~m}$ depth) of Curaçao and Bonaire declined from an average of $32.6 \%$ in the early 1970 s to a mere $9.2 \%$ in 2013 (Chapter II). Similarly, mean coral cover across the leeward reef terrace of Bonaire was reduced to 12\% in 2017 (Chapter VI). Other calcifyers, including crustose coralline algae and hydrocorals are shown to respond correspondingly to the changed conditions on these reefs (Chapter II \& Chapter VI). Through human interference on global and local scales, these calcifying organisms have lost their competitive advantage over fast growing opportunistic organisms such as algal turfs and macroalgae (e.g. Hughes et al. 1999; Vermeij et al. 2010). One of the disturbing findings of this thesis is that the previously ascertained 'coral-algal phase shift' (Hughes 1994; Jackson et al. 2014) not only occurred on the shallow reef, but down to depths of at least $40 \mathrm{~m}$. The 40 -year time series provides compelling and troubling support to challenge (Bak et al. 2005; Bongaerts et al. 2010) the hypothesis postulating that upper mesophotic reefs largely escape anthropogenic impact and as such can serve as refugia to corals and other key organisms (Glynn 1996).

A major novelty presented in this thesis is the first quantitative description of the 'rise of benthic cyanobacterial mats (BCMs)' as a major constituent of coral reef biotic communities (Chapter II). At present, a wide array of literature exists focussing on the various aspects of the shift from coral to algae as the prominent functional biotic group within reef habitats (see Jackson et al. 2014). In part due to the conspicuousness of this development, relatively little attention has been paid to temporal trajectories of other reef biota, including sponges and benthic cyanobacteria. These bacteria have consistently been present in coral reef habitats (Charpy et al. 2012; Brocke et al. 2018; Ford et al. 2018), but not in densities as we are observing them nowadays on the reef slope (mean cover of $20 \%$ ). They managed to remain below the scientific radar since they are often neglected or lumped together with algae in benthic surveys. Consequently, our understanding of the impact of this new player is limited. BCMs, however, are known to display a variety of harmful characteristics that impair essential reef functions and affect other reef biota 
(Carlton and Richardson 1995; Nagle and Paul 1998; Ritson-Williams et al. 2005; Kuffner et al. 2006; Brocke et al. 2015a; De Bakker et al. 2016a).

Much like with fresh water blooms of planktonic cyanobacteria (Paerl and Huisman 2008; Huisman et al. 2018), reef dwelling cyanobacteria appear to proliferate under eutrophic, warm conditions (Brocke et al. 2015b; Den Haan 2015). Although the increased abundance of BCMs, as presented here, is confirmed on reefs throughout the Caribbean as well as the Pacific (Charpy et al. 2012; Ford et al. 2018), their local manifestation and prevalence is still highly dynamic (personal observation). The influence of physical disturbance (e.g. wave action, storms), seasonality, temperature anomalies and the increased availability of nutrients and organic matter on their metabolism should be further elucidated to understand and predict the impact that these efficient and adaptive blooming-like bacterial assemblages have on reef ecosystems. Variation in aforementioned factors, in particular hydrological disturbance in shallow reef zones and fluctuations in water temperature (e.g. due to seasonality), may for instance explain why relatively little BCMs are encountered in the elaborate survey reefs around Bonaire in 2014 and 2017 (Chapter VI).

Although BMCs may not be a dominant component on the reef terrace at this point, the shallowest reef zones have nonetheless undergone a significant transformation over the past decades (Chapter VI). Until the outbreak of the white band disease, extensive fields of Acropora cervicornis dominated this part of the reef (Bak 1977; Van Duyl 1985). Currently, however, it is characterized by Acropora rubble and old remnants of deceased coral colonies interspersed with sandy patches (Chapter VI). Algal turf covering the hard carbonate substrata is the most prominent biota across virtually the entire reef terrace. The distribution and abundance of other biota appears to be related to variable local natural conditions and the local severity of anthropogenic exerted stress. Relatively high coral and gorgonian cover was found in regions with marginal human interference and substantial macroalgal fields at exposed sites in the north. Sponges, on the other hand, were found wherever hard substratum was available, albeit accounting for relatively little cover (Chapter VI). Temporal analysis of their cover did show that sponges have increased in abundance across all studied depths since the 1970s (Chapter II). Considering their often 3-dimensional morphology, however, percent cover poorly reflects what their increased abundance means in terms of accumulated sponge biomass on the reef. Sponges have historically been an important component on Caribbean reefs, fulfilling multiple key functions within the ecosystem such as energy recycling, cementation and bioerosion (Diaz and Rützler 2001; De Goeij et al. 2013). We encourage investigation that elucidates the role sponges fulfil within the present-day reef habitat configurations and in possible feedback interactions with algae and BCMs (e.g. recycling of dissolved, particulate organic matter and nutrients). The still patchy distribution and unsteady prevalence of BCMs might indicate that we presently reside on the verge of entering a new alarming phase in the degradation of reef systems, a phase characterized by miscellaneous abiotic substrata (rubble, sand and remnants of corals) covered with BCMs, (turf)algae, sponges and a few resilient corals. (Figure 7.1). 


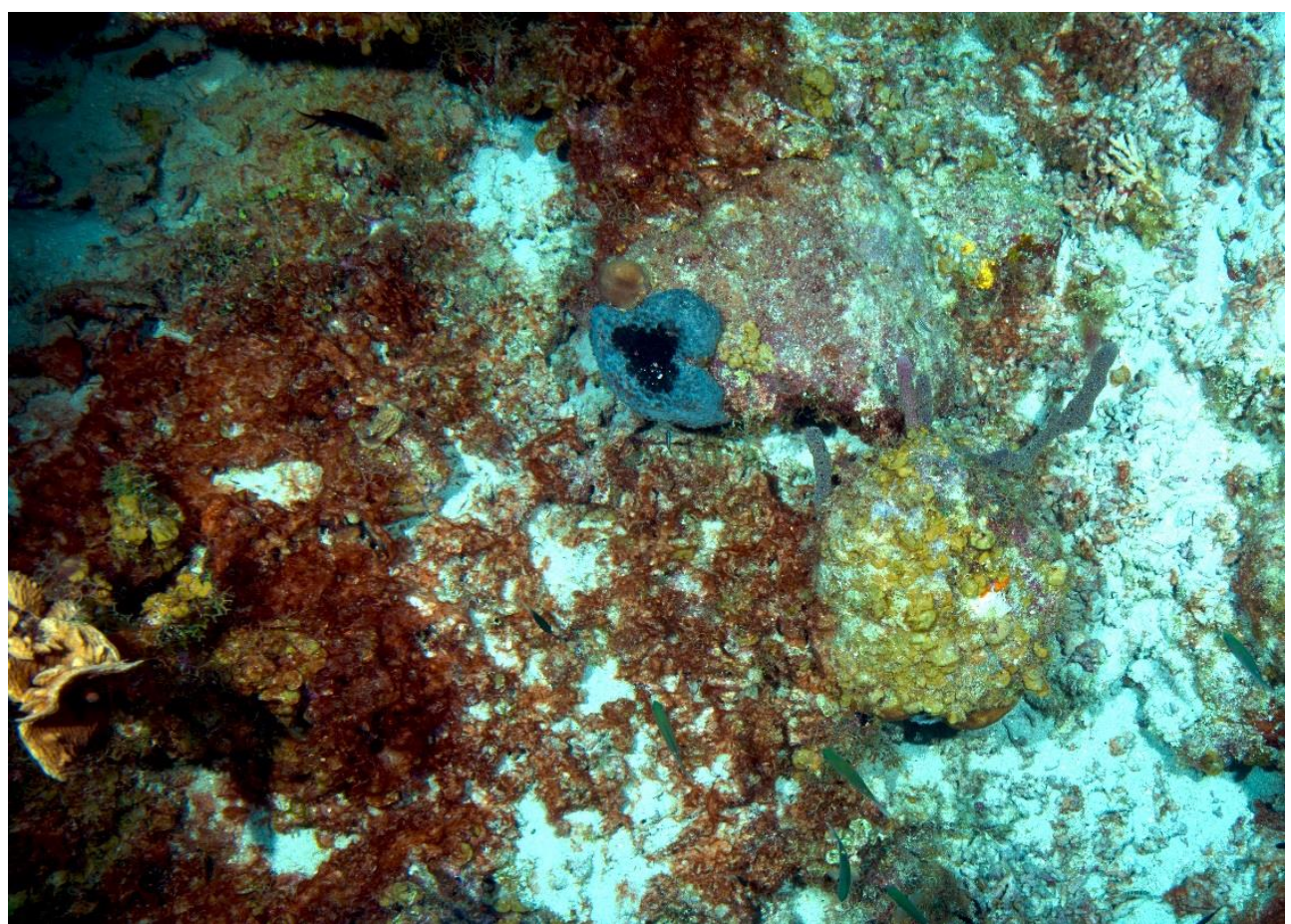

Figure 7.1. Will this be the future of Caribbean coral reefs? Detail of a permanent quadrat on Curaçao at $20 \mathrm{~m}$ depth, October 2016. Photo credits: Dr. Erik Meesters.

\section{Flattening of the reef}

A second major focus of this thesis has been the decline in net calcium carbonate $\left(\mathrm{CaCO}_{3}\right)$ production and consequential loss of structural complexity on coral reefs due to benthic community rearrangements (Chapter III to V). Alongside the ubiquitous decline in cover of bioaccreting organisms in recent decades, historical dominance of large reef-constructing corals (Orbicella spp., Acropora spp.) was interrupted as well (Pandolfi and Jackson 2006) and coral assemblages have often shifted to dominance of smaller opportunistic coral species (Chapter III). Most notably, the loss of the greater part of Acropora spp. colonies in the shallow reef means a substantial decline in carbonate production. Moreover, remaining carbonate structures are more rapidly eroded because of the relative success of benthic bioeroding organisms in the current reef environment (Glynn and Manzello 2015; Schönberg et al. 2017). The theory that these synergetic developments have resulted in a substantial decline in biogenic reef accretion on Caribbean reefs is widely supported (e.g. Alvarez-Filip et al. 2009; Alvarez-Filip et al. 2013; Perry et al. 2013; Januchowski-Hartley et al. 2017). Yet, this thesis presents the first quantitative confirmation that carbonate production by the coral assemblage has indeed substantially declined over time (Chapter III).

The data presented in Chapter $\mathbf{V}$ indicate that a large part of the leeward reef of Bonaire is indeed currently eroding away, ultimately halting net vertical accretion of the shallow terrace. The applied methodology as outlined by Perry et al (2012) and Perry et al. (2015) appears to 
yield reliable estimates for the maximum reef accretion potential. The methods, however, understandably include various assumptions and simplifications suggesting there is room for improvement. Chapter IV indicates that the capacity of excavating sponges to erode carbonate substrate varies significantly among the different species. Accordingly, we have implemented species-specific rates for sponge bioerosion to, more appropriately, estimate the contribution of excavating sponges to gross bioerosion. Despite the fact that some imperfections still exist in the applied methodology it is evident that many reef sites around Bonaire and Curaçao are struggling to maintain positive net reef accretion. That consequently the perseverance and future development of structurally complex reefs is negatively affected (Chapter $\mathbf{V}$ ) has extensive consequences for essential reef functions and for us humans as well. Many reef fish and invertebrates rely on architectural complexity for cover against predators (Beukers and Jones 1998). Fish stocks and species richness, including that of commercially important fish such as groupers and snappers, indeed appears to be diminished, most notably in areas without a structurally complex habitat (Newman et al. 2015; Chapter V \& Chapter VI). Perhaps even more concerning is the finding that the majority of the shallow reef sites around Bonaire will, under the current conditions, not be able to track even the most optimistic sea level rise scenarios (Chapter V). Because of this, coastlines will experience more erosion and inundation will become more frequent (Moberg and Rönnbäck 2003; Harris et al. 2018; Storlazzi et al. 2018). The notion that the reefs of Bonaire allegedly rank among the least degraded within the greater Caribbean region (Jackson et al. 2014), suggests that these troubling findings will in likelihood apply to many other islands in the tropical Atlantic.

Nonetheless, the findings presented in this thesis indicate that some of the shallow northern reefs of Bonaire appear to be well 'persevered'. They are still characterized by high coral cover of framework-building species (most notably Orbicella spp.), considerable architectural complexity and a substantial abundance of fish (Chapter V \& Chapter VI). Reefs towards the south (with exception of the southernmost point) and on the reef slope, however, are often heavily degraded. Spatial disparity in the degree of ecological degradation (Chapter $\mathbf{V} \&$ Chapter VI) on coral reefs is not a complete novelty. The fact, however, that this variation is found on such a small scale is highly relevant. It promptly touches on a sore point, namely: survey sample size and shifting baselines. Throughout the available literature, local findings are often tentatively extrapolated to larger spatial scales. I acknowledge that this happens within the scope of this thesis as well. This is virtually inherent to science. The coral reefs in the southern Caribbean are generally considered to be in a better ecological condition than elsewhere in the Caribbean (Jackson et al. 2014), while in fact large stretches of the reefs around Bonaire and Curaçao are approaching or already experiencing collapse of reef ecosystem functioning (Figure 7.1). Worryingly, these heavily degraded reefs are found, in particular, in front of densely populated and inundation sensitive areas. The potential spatial variability as presented in this thesis, thus, indicates that caution is advised when drawing large-scale conclusions based on just a few sites or transects. Transect placement can cause significant bias with regards to the condition of reefs on an island or within a wider geographical region. Although it seems that virtually all reef sites described in this thesis have experienced some degree of ecological 
degradation compared to pre-1980 conditions, some, albeit sporadically, near-pristine reef configurations were indeed still encountered (Figure 7.2).

\section{Modest hope for Caribbean coral reefs}

Parallel to the sketched gloomy confirmation that most present-day coral reef ecosystems are in a historically poor condition, the findings presented in this thesis also signify a humble message of hope for preservation and recovery. The survival of some relatively well-developed seemingly 'healthy' reefs around Curaçao and Bonaire is reassuring (Figure 7.2). Their 'survival' suggests that, under favourable local conditions, reefs are able to cope with the present-day global environment. Of course, this is hardly a guarantee that these reefs will endure, certainly not when climate change and local human impact continue to intensify. Nonetheless, it is conspicuous that relatively non-degraded reefs exist in areas with little to no human activity (Chapter III \& Chapter VI). Although this may seem evident, it has rarely been quantified on a scale as displayed in this thesis. This finding, in combination with the observation that some of the least degraded reefs were encountered in marine reserves, amplifies the need for appropriate and adequate local management of coral reefs. We should learn from the relatively good reefs of Curaçao and Bonaire for management of the wider Caribbean region. Not solely in the interest of preserving marine biodiversity, but also to ensure continuation of the goods and services provided by the reef to us.

Furthermore, the first outcome of the newly started time series presented in this thesis (Chapter VI) may indicate cautious signs of stabilization of the degradation of the shallow reef habitat (< $15 \mathrm{~m}$ depth). Granted that, on itself, the temporal robustness of time series is at present limited, by comparing the findings to dedicated surveys conducted by Sommer et al. (2011) in 2008 and the data presented in Chapter II and Chapter III it could be argued that such a stabilizing trend may have been occurring already for the past decade. It is difficult to pinpoint the exact reasons for this apparent stabilization, because it is likely to be the result of a combination of factors. During the period for the relative steadiness and even marginal recovery of several reef features, the reefs of Bonaire have not suffered large-scale disturbances such as mass bleaching or hurricanes. In addition, Bonaire has implemented appreciable management of coral reef resources, including the establishment of the National Marine Park (BNMP) in 1979, the designation of two fish reserves (no fishing allowed) and two dive reserves (no diving or snorkelling allowed) in 2008. Furthermore, the majority of the island has recently been connected to a communal sewer. It is not implausible that such implementations and the lack of large-scale disturbance, in combination with the selection of more resistant coral species (e.g. Porites astreoides, Agaricia agaricites and the various Madracis spp.) or genetic morphotypes, aid in slowly restoring some degree of reef resilience. A more sceptical view would be that any form of stabilization is the result of a reached minimum cover of r-selected opportunistic coral species that could still endure even under worsening condition. Following this analogy, coral cover may have declined to a point where percentage decline has become hardly noticeable. Whether this is the case or whether reefs will indeed start a hesitant recovery has to be confirmed. Similar trends, however, are seen throughout the greater Caribbean region (personal observation). As such, I strongly believe that improvement of local conditions can increase the 
capacity of coral reef ecosystems to cope with stressors that act on a global scale, including climate change. Both Curaçao and Bonaire at present still harbour reef sections, distant from high human impact, that are in a considerably good condition. As previously stated, it is unlikely that we will be able to restore reefs to pre-1980 configurations (Hughes et al. 2017), but it is not too late to preserve and restore essential ecological functions and trophic flows. What is shown evidently throughout this thesis is that resilient reefs can exist when negative local human impact is minimized. We as humans have the power to initiate change, but we need to act now and improve conditions on a global scale, but certainly on a local scale as well.

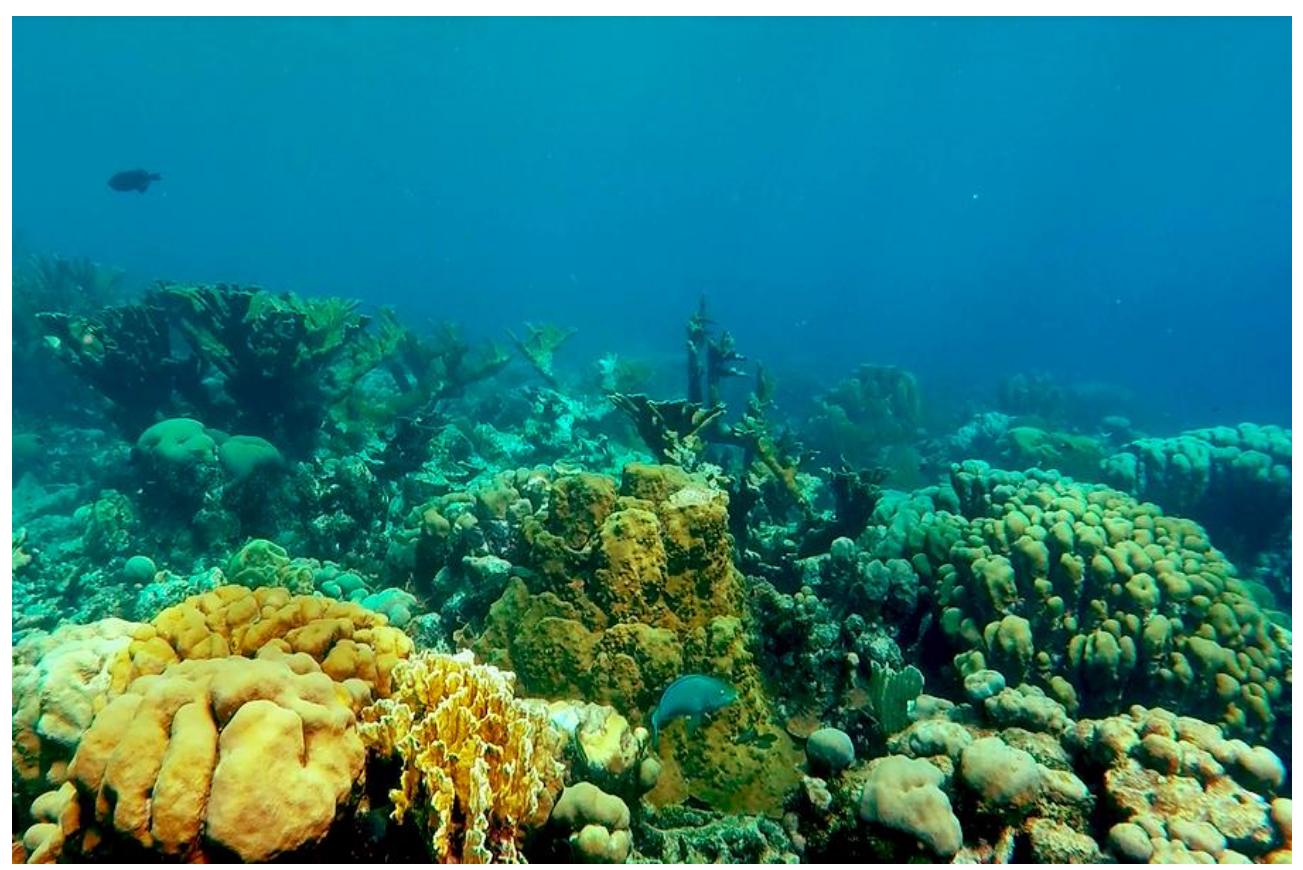

Figure 7.2. Or will we aim to make coral reefs look more like this? Reef in the northern part of Bonaire, October 2016. Credits: Didier de Bakker. 
Chapter VII 


\section{List of references}

Achlatis M, van der Zande RM, Schönberg CH, Fang JK, Hoegh-Guldberg O, Dove S (2017) Sponge bioerosion on changing reefs: ocean warming poses physiological constraints to the success of a photosymbiotic excavating sponge. Scientific Reports 7(1): 10705

Acker KL, Risk MJ (1985) Substrate destruction and sediment production by the boring sponge Cliona caribbaea on Grand Cayman Island. Journal of Sedimentary Research 55(5): 705-711

Alemu JB, Clement Y (2014) Mass coral bleaching in 2010 in the southern Caribbean. PLoS one 9(1): e83829

Alexander BE, Liebrand K, Osinga R, van der Geest HG, Admiraal W, Cleutjens JP, Schutte B, Verheyen F, Ribes M, van Loon E, de Goeij JM (2014) Cell turnover and detritus production in marine sponges from tropical and temperate benthic ecosystems. PLoS One 9(10): e109486

Alvarez-Filip L, Dulvy NK, Gill JA, Côté IM, Watkinson AR (2009) Flattening of Caribbean coral reefs: regionwide declines in architectural complexity. Proceedings of the Royal Society of London B: Biological Sciences 276(1669): 3019-3025

Alvarez-Filip L, Dulvy NK, Côté IM, Watkinson AR, Gill JA (2011a) Coral identity underpins architectural complexity on Caribbean reefs. Ecological Applications 21(6): 2223-2231

Alvarez-Filip L, Gill JA, Dulvy NK, Perry AL, Watkinson AR, Côté IM (2011b) Drivers of region-wide declines in architectural complexity on Caribbean reefs. Coral Reefs 30(4): 1051-1060

Alvarez-Filip L, Côté I M, Gill JA, Watkinson AR, Dulvy NK (2011c) Region-wide temporal and spatial variation in Caribbean reef architecture: is coral cover the whole story? Global Change Biology, 17(7): 24702477

Alvarez-Filip L, Carricart-Ganivet JP, Horta-Puga G, Iglesias-Prieto R (2013) Shifts in coral-assemblage composition do not ensure persistence of reef functionality. Scientific Reports 3: 3486

Anderberg MR (1973) Cluster analysis for applications. Academic Press, New York

Anderson MJ (2001) A new method for non-parametric multivariate analysis of variance. Austral ecology 26(1): $32-46$

Anderson MJ, Thompson AA (2004) Multivariate control charts for ecological and environmental monitoring. Ecological Applications 14(6): 1921-1935

Anderson R, Morrall C, Jossart J, Nimrod S, Bolda E, Musser K, Berg C, Balza R (2014) Marine protected area monitoring in the nearshore waters of Grenada, Eastern Caribbean: benthic cover and fish populations. Revista de Biologia Tropical 62: 273-286

Andersson AJ, Gledhill D (2013) Ocean acidification and coral reefs: effects on breakdown, dissolution, and net ecosystem calcification. Annual Review of Marine Science 5: 321-348

Arnold SN, Steneck RS, Mumby PJ (2010) Running the gauntlet: inhibitory effects of algal turfs on the processes of coral recruitment. Marine Ecology Progress Series 414: 91-105

Aronson RB, Precht WF (2001) White-band disease and the changing face of Caribbean coral reefs. In The ecology and etiology of newly emerging marine diseases. Springer, Dordrecht, pp25-38

Aronson RB, Precht WF, Toscano M, Koltes KH (2002a) The 1998 bleaching event and its aftermath on a coral reef in Belize. Marine Biology 141(3): 435-447

Aronson RB, Macintyre IG, Precht WF, Murdoch TJT, Wapnick CM (2002b) The expanding scale of species turnover events on coral reefs in Belize. Ecological Monographs 72(2): 233-249

Bak RPM (1975) Ecological aspects of the distribution of reef corals in the Netherlands Antilles. Bijdragen tot de Dierkunde 45(2): 181-190

Bak RPM (1976) The growth of coral colonies and the importance of crustose coralline algae and burrowing sponges in relation with carbonate accumulation. Netherlands Journal of Sea Research 10(3): 285-337

Bak RPM (1977) Coral Reefs and Their Zonation in Netherlands Antilles: Modern and Ancient Reefs. AAPG Studies in Geology 4: 3-16

Bak RPM, Engel MS (1979) Distribution, abundance and survival of juvenile hermatypic corals (Scleractinia) and the importance of life history strategies in the parent coral community. Marine Biology 54(4): 341-352

Bak RPM, Luckhurst BE (1980) Constancy and change in coral reef habitats along depth gradients at Curaçao. Oecologia 47(2): 145-155 
Bak RPM, Criens SR (1981) Survival after fragmentation of colonies of Madracis mirabilis, Acropora palmata and A. cervicornis (Scleractinia) and the subsequent impact of a coral disease. Proceedings of the 4th International Coral Symposium 2: 221-227

Bak RPM, Sybesma J, Van Duyl FC (1981) The Ecology of the tropical compound ascidian Trididemnum solidum. 11. Abundance, Growth and Survival. Marine Ecology Progress Series 6: 43-52

Bak RPM, Carpay MJE, De Ruyter Van Steveninck ED (1984) Densities of the sea urchin Diadema antillarum before and after mass mortalities on the coral reefs on Curaçao. Marine Ecology Progress Series. Oldendorf 17(1):105-108

Bak RPM, Nieuwland G (1995) Long-term change in coral communities along depth gradients over leeward reefs in the Netherlands Antilles. Bulletin of Marine Science 56(2): 609-619

Bak RPM, Lambrechts DYM, Joenje M, Nieuwland G, Van Veghel MLJ (1996) Long-term changes on coral reefs in booming populations of a competitive colonial ascidian. Marine Ecology Progress Series 133: $303-$ 306

Bak RPM, Meesters EH (1998) Coral population structure: the hidden information of colony size-frequency distributions. Marine Ecology Progress Series 162: 301-306

Bak RPM, Nieuwland G, Meesters EH (2005) Coral reef crisis in deep and shallow reefs: 30 years of constancy and change in reefs of Curaçao and Bonaire. Coral reefs 24(3): 475-479

Bellwood DR, Hoey AS, Choat JH (2003) Limited functional redundancy in high diversity systems: resilience and ecosystem function on coral reefs. Ecology letters 6(4): 281-285

Bellwood DR, Hughes TP, Folke C, Nyström M (2004) Confronting the coral reef crisis. Nature 429(6994): 827833

Bender D, Diaz-Pulido G, Dove S (2014) Warming and acidification promote cyanobacterial dominance in turf algal assemblages. Marine Ecology Progress Series 517: 271-284

Bender MA, Knutson TR, Tuleya RE, Sirutis JJ, Vecchi GA, Garner ST, Held IM (2010) Modeled impact of anthropogenic warming on the frequency of intense Atlantic hurricanes. Science 327(5964): 454-458

Beukers JS, Jones GP (1998) Habitat complexity modifies the impact of piscivores on a coral reef fish population. Oecologia 114(1): 50-59

Blanchon P, Iglesias-Prieto R, Jordan-Dahlgren E, Richards S (2010) Arrecifes de coral y cambio climático: vulnerabilidad de la zona costera del estado de Quintana Roo. In Vulnerabilidad de las zonas costeras mexicanas ante el cambio climático. Universidad Autónoma de Campeche, Mexico pp229-248

Bohnsack JA, Harper DE (1988) Length-weight relationships of selected marine reef fishes from the southeastern United States and the Caribbean. National Fish and Wildlife Service, Miami

Bongaerts P, Ridgway T, Sampayo E, Hoegh-Guldberg O (2010) Assessing the 'deep reef refugia'hypothesis: focus on Caribbean reefs. Coral reefs 29(2): 309-327

Borcard D, Gillet F, Legendre P (2011) Spatial analysis of ecological data. In Numerical ecology with R. Springer, New York, pp227-292

Bosscher H, Schlager W (1992) Computer simulation of reef growth. Sedimentology 39:503-512

Bray JR, Curtis JT (1957) An ordination of the upland forest communities of southern Wisconsin. Ecological monographs 27(4): 325-349

Breland JA, Byrne RH (1993) Spectrophotometric procedures for determination of sea water alkalinity using bromocresol green. Deep Sea Research Part I: Oceanographic Research Papers 40(3): 629-641

Bries JM, Debrot AO, Meyer DL (2004) Damage to the leeward reefs of Curaçao and Bonaire, Netherlands Antilles from a rare storm event: Hurricane Lenny, November 1999. Coral Reefs 23(2): 297-307

Brocke HJ, Polerecky L, De Beer D, Weber M, Claudet J, Nugues MM (2015a) Organic Matter Degradation Drives Benthic Cyanobacterial Mat Abundance on Caribbean Coral Reefs. PloS One 10(5): e0125445

Brocke HJ, Wenzhoefer F, De Beer D, Mueller B, Van Duyl FC, Nugues MM (2015b) High dissolved organic carbon release by benthic cyanobacterial mats in a Caribbean reef ecosystem. Scientific Reports 5: 8852

Brocke HJ, Piltz B, Herz N, Abed RM, Palinska KA, John U, den Haan J, de Beer D, Nugues MM (2018) Nitrogen fixation and diversity of benthic cyanobacterial mats on coral reefs in Curaçao. Coral Reefs 37(3): 861-874

Bruckner AW, Bruckner RJ (2006) The recent decline of Montastraea annularis (complex) coral populations in western Curaçao: a cause for concern? Revista de Biologia Tropical 54: 45-58

Bruno JF, Bertness MD (2001) Habitat modification and facilitation in benthic marine communities. In Marine community ecology, Sinauer. Sunderland, pp201-218 
Bruno JF, Petes LE, H.C. D, Hettinger A (2003) Nutrient enrichment can increase the severity of coral diseases. Ecology Letters 6(12): 1056-1061

Bruno JF, Sweatman H, Precht WF, Selig ER, Schutte VGW (2009) Assessing evidence of phase shifts from coral to macroalgal dominance on coral reefs. Ecology 90(6): 1478-1484

Budd AF, Johnson KG, Stemann TA, Jackson JBC (1996) Plio-Pleistocene turnover and extinctions in the Caribbean reef coral fauna. In Evolution and environment in tropical America. University of Chicago press, Chicago \& London pp168-204

Budd AF, Johnson K, Stemann T, Tompkins B (1999) Pliocene to Pleistocene reef coral assemblages in the Limón group of Costa Rica. Gremios de arrecifes de coral del Plioceno al Pleistoceno en el grupo de Limón de Costa Rica. Bulletins of American Paleontology 357: 119-158

Budd AF (2000) Diversity and extinction in the Cenozoic history of Caribbean reefs. Coral Reefs 19(1): 25-35

Budd AF, Fukami H, Smith ND, Knowlton N (2012) Taxonomic classification of the reef coral family Mussidae (Cnidaria: Anthozoa: Scleractinia). Zoological Journal of the Linnean Society 166(3): 465-529

Burkepile DE, Hay ME (2008) Herbivore species richness and feeding complementarity affect community structure and function on a coral reef. Proceedings of the National Academy of Sciences U.S.A 115: 1620116206

Buth L, Ras J (1992) Inventory of the land-based sources of marine pollution Netherlands Antilles. Department of Public Works of Curaçao and Environmental Service Curaçao; prepared for CEPPOL Program, IOC-UNEP $\mathrm{CAR} / \mathrm{RCU}$

Calcinai B, Azzini F, Bavestrello G, Gaggero L, Cerrano C (2007) Excavating rates and boring pattern of Cliona albimarginata (Porifera: Clionaidae) in different substrata. In Porifera research: biodiversity, innovation and sustainability Procedings of the 7th International Sponge Symposium pp255-263

Carballo JL, Bautista E, Nava H, Cruz-Barraza JA, Chávez JA (2013) Boring sponges, an increasing threat for coral reefs affected by bleaching events. Ecology and Evolution 3(4): 872-886

Carballo JL, Ovalle-Beltrán H, Yáñez B, Bautista-Guerrero E, Nava-Bravo H (2017) Assessment of the distribution of sponge chips in the sediment of East Pacific Ocean reefs. Marine Ecology 38(1): e12390

Carlton RG, Richardson LL (1995) Oxygen and sulfide dynamics in a horizontally migrating cyanobacterial mat: black band disease of corals. FEMS Microbiology Ecology 18(2): 155-162

Carpenter KE, Abrar M, Aeby G, Aronson RB, Banks S, Bruckner A, Chiriboga A, Cortés J, Delbeek JC, DeVantier L (2008) One-third of reef-building corals face elevated extinction risk from climate change and local impacts. Science 321: 560-563

Chambers JM, Hastie TJ (1992) Linear models. In Statistical Models in S. Wadsworth \& Brooks/Cole Pub, Pacific Grove, California, pp95-144

Charpy L, Casareto BE, Langlade M, Suzuki Y (2012) Cyanobacteria in coral reef ecosystems: a review. Journal of Marine Biology 2012: Article ID 259571

Chaves-Fonnegra A, Zea S (2007) Observations on reef coral undermining by the Caribbean excavating sponge Cliona delitrix (Demospongiae, Hadromerida). Porifera research: biodiversity, innovation and sustainability. Série Livros 28: 247-264

Chaves-Fonnegra A, Zea S (2011) Coral colonization by the encrusting excavating Caribbean sponge Cliona delitrix. Marine Ecology 32(2): 162-173

Chaves-Fonnegra A, Feldheim KA, Secord J, Lopez JV (2015) Population structure and dispersal of the coralexcavating sponge Cliona delitrix. Molecular ecology 24(7): 1447-1466

Chollett I, Mumby PJ (2012) Predicting the distribution of Montastraea reefs using wave exposure. Coral Reefs 31(2): 493-503.

Clarke KR, Warwick RM, Brown BE (1993) An index showing breakdown of seriation, related to disturbance, in a coral-reef assemblage. Marine Ecology Progress Series 102: 153-160

Clarke KR, Warwick RM (1994) An approach to statistical analysis and interpretation. Change in Marine Communities 2: 117-143

Clarke RK, Somerfield PJ, Airoldi L, Warwick RM (2006) Exploring interactions by second-stage community analyses. Journal of Experimental Marine Biology and Ecology 338(2): 179-192

Coates AG, Jackson JBC, Collins LS, Cronin TM, Dowsett HJ, Bybell LM, Jung P, Obando JA (1992) Closure of the Isthmus of Panama: the near-shore marine record of Costa Rica and western Panama. Geological Society of America Bulletin 104(7): 814-828 
Epilogue

Cobb WR (1969) Penetration of calcium carbonate substrates by the boring sponge, Cliona. American Zoologist 9(3): 783-790

Connell JH (1978) Diversity in tropical rain forests and coral reefs. Science 199(4335): 1302-1310

Connell SD, Foster MS, Airoldi L (2014) What are algal turfs? Towards a better description of turfs. Marine Ecology Progress Series 495: 299-307

Copper P (1988) Ecological succession in Phanerozoic reef ecosystems: is it real? Palaios 3: 136-151

Costanza R, d'Arge R, De Groot R, Farber S, Grasso M, Hannon B, Limburg K, Naeem S, O'neill RV, Paruelo J (1997) The value of the world's ecosystem services and natural capital. Nature 387(6630): 253-260

Cramer KL, O'Dea A, Clark TR, Zhao J-x, Norris RD (2017) Prehistorical and historical declines in Caribbean coral reef accretion rates driven by loss of parrotfish. Nature communications 8: 14160

Darling ES, Alvarez-Filip L, Oliver A, McClanahan TR, Côté IM (2012) Evaluating life-history strategies of reef corals from species traits. Ecology Letters 15(12): 1378-1386

De Bakker DM, Meesters EH, Van Bleijswijk JDL, Luttikhuizen PC, Breeuwer HJAJ, Becking LE (2016a) Population Genetic Structure, Abundance, and Health Status of Two Dominant Benthic Species in the Saba Bank National Park, Caribbean Netherlands: Montastraea cavernosa and Xestospongia muta. PloS One 11: e0155969

De Bakker DM, Meesters EH, Bak RPM, Nieuwland G, Van Duyl FC (2016b) Long-term shifts in coral communities on shallow to deep reef slopes of Curaçao and Bonaire: are there any winners? Frontiers in Marine Science 3: 247

De Bakker DM, Van Duyl FC, Bak RPM, Nugues MM, Nieuwland G, Meesters EH (2017) 40 Years of benthic community change on the Caribbean reefs of Curaçao and Bonaire: the rise of slimy cyanobacterial mats. Coral Reefs 36(2): 355-367

De Bakker DM, Webb AE, van den Bogaart LA, van Heuven SMAC, Meesters EH, van Duyl FC (2018) Quantification of chemical and mechanical bioerosion rates of six Caribbean excavating sponge species found on the coral reefs of Curaçao. PloS One 13(5): e0197824

De Goeij JM, Van Oevelen D, Vermeij MJA, Osinga R, Middelburg JJ, De Goeij AFPM, Admiraal W (2013) Surviving in a marine desert: the sponge loop retains resources within coral reefs. Science 342(6154): 108-110

De Graaf M, Houtepen E, Tichelaar E, Miller DCM, Brunel T, Nagelkerke LAJ (2016) Status and trends reef fish and coastal fisheries Bonaire (Caribbean Netherlands): report card 2014-2015. Wageningen University \& Research Rapport C087(16): 1-71

De León R, Vane K, Bertuol P, Chamberland VC, Simal F, Imms E, Vermeij, MJ (2013) Effectiveness of lionfish removal efforts in the southern Caribbean. Endangered Species Research, 22(2): 175-182

De Meyer K, Park BM, Box F (1998) Bonaire, Netherlands Antilles. CARICOMP_Caribbean coral reef, seagrass and mangrove sites Coastal Region and Small Island Papers 3: 141-149

De Ruyter Van Steveninck EDD, Bak RPM (1986) Changes in abundance of coral-reef bottom components related to mass mortality of the sea-urchin Diadema Antillarum. Marine Ecology Progress Series 34: 87-94

De'ath G (2002) Multivariate regression trees: a new technique for modeling species-environment relationships. Ecology 83(4): 1105-1117

De'ath G (2006) Mvpart: multivariate partitioning R package version 1.2-4. Rpart by TM Therneau and B. Atkinson. $R$ port of rpart by B. Ripley. Some routines from vegan by J. Oksanen

De'ath G, Fabricius KE, Sweatman H, Puotinen M (2012) The 27-year decline of coral cover on the Great Barrier Reef and its causes. Proceedings of the National Academy of Sciences USA 109: 17995-17999

Den Haan J (2015) Effects of nutrient enrichment on the primary producers of a degraded coral reef. Faculteit der Natuurwetenschappen, Wiskunde en Informatica PhD thesis, University of Amsterdam, pp38-58

Derraik JG (2002) The pollution of the marine environment by plastic debris: a review. Marine Pollution Bulletin 44(9): 842-852

Diaz MC, Rützler K (2001) Sponges: an essential component of Caribbean coral reefs. Bulletin of Marine Science 69(2): 535-546

Done TJ, DeVantier LM, Turak E, Fisk DA, Wakeford M, Van Woesik R (2010) Coral growth on three reefs: development of recovery benchmarks using a space for time approach. Coral Reefs 29(4): 815-833

Donner SD, Knutson TR, Oppenheimer M (2007) Model-based assessment of the role of human-induced climate change in the 2005 Caribbean coral bleaching event. Proceedings of the National Academy of Sciences 104(13): 5483-5488 
Eakin CM (1996) Where have all the carbonates gone? A model comparison of calcium carbonate budgets before and after the 1982-1983 El Nino at Uva Island in the eastern Pacific. Coral Reefs 15(2): 109-119

Eakin CM, Morgan JA, Heron SF, Smith TB, Liu G, Alvarez-Filip L, Baca B, Bartels E, Bastidas C, Bouchon C (2010) Caribbean corals in crisis: record thermal stress, bleaching, and mortality in 2005. PloS One 5: e13969

Edinger EN, Risk MJ (2000) Reef classification by coral morphology predicts coral reef conservation value. Biological Conservation 92(1): 1-13

Edmunds PJ, Elahi R (2007) The demographics of a 15-year decline in cover of the Caribbean reef coral Montastraea annularis. Ecological Monographs 77(1): 3-18

Edwards CB, Friedlander AM, Green AG, Hardt MJ, Sala E, Sweatman HP, Williams ID, Zgliczynski B, Sandin SA, Smith JE (2014) Global assessment of the status of coral reef herbivorous fishes: evidence for fishing effects. Procedings of the Royal Society B 281(1774): 20131835

Engel M, Brückner H, Messenzehl K, Frenzel P, May SM, Scheffers A, Scheffers S, Wennrich V, Kelletat D (2012) Shoreline changes and high-energy wave impacts at the leeward coast of Bonaire (Netherlands Antilles). Earth, Planets and Space 64(10): 9

Erez J, Reynaud S, Silverman J, Schneider K, Allemand D (2011) Coral calcification under ocean acidification and global change. In Coral reefs: An Ecosystem in Transition. Springer, New York, pp151-176

Estes JA, Palmisano JF (1974) Sea otters: their role in structuring nearshore communities. Science 185(4156): 10581060

Eyre BD, Cyronak T, Drupp P, De Carlo EH, Sachs JP, Andersson AJ (2018) Coral reefs will transition to net dissolving before end of century. Science 359(6378): 908-911.

Fabricius KE (2005) Effects of terrestrial runoff on the ecology of corals and coral reefs: review and synthesis. Marine pollution bulletin 50(2): 125-146

Fabricius KE, De'ath G, McCook L, Turak E, Williams DM (2005) Changes in algal, coral and fish assemblages along water quality gradients on the inshore Great Barrier Reef. Marine pollution bulletin 51(14): 384-398

Faith DP, Minchin PR, Belbin L (1987). Compositional dissimilarity as a robust measure of ecological distance. Vegetatio 69(1-3): 57-68

Fang JK, Schönberg CHL, Kline DI, Hoegh-Guldberg O, Dove S (2013a) Methods to quantify components of the excavating sponge Cliona orientalis Thiele, 1900. Marine Ecology 34(2): 193-206

Fang JK, Mello-Athayde MA, Schönberg CH, Kline DI, Hoegh-Guldberg O, Dove S (2013b) Sponge biomass and bioerosion rates increase under ocean warming and acidification. Global change biology 19(12): 35813591

Fang JK, Schönberg CHL, Mello-Athayde MA, Hoegh-Guldberg O, Dove S (2014) Effects of ocean warming and acidification on the energy budget of an excavating sponge. Global change biology 20(4): 1043-1054

Fang JK, Schönberg CHL, Hoegh-Guldberg O, Dove S (2016) Day-night ecophysiology of the photosymbiotic bioeroding sponge Cliona orientalis Thiele, 1900. Marine Biology 163(5): 1-12

Ferrario, F, Beck MW, Storlazzi CD, Micheli F, Shepard CC, Airoldi, L (2014) The effectiveness of coral reefs for coastal hazard risk reduction and adaptation. Nature communications 5: 3794

Flato G, Marotzke J, Abiodun B, Braconnot P, Chou SC, Collins WJ, Cox P, Driouech F, Emori S, Eyring V, Forest C, Glecker P, Guilyardi E, Jacob C, Kattsov V, Rummukainen M (2013) Evaluation of climate models. In: Climate change 2013: the physical science basis. Contribution of working group I to the fifth assessment report of the intergovernmental panel on climate change. University Press, Cambridge

Ford AK, Bejarano S, Nugues MM, Visser PM, Albert S, Ferse SC (2018) Reefs under siege - the rise, putative drivers, and consequences of benthic cyanobacterial mats. Frontiers in Marine Science 5: 18

Gardner TA, Côté IM, Gill JA, Grant A, Watkinson AR (2003) Long-term region-wide declines in Caribbean corals. Science 301(5635): 958-960

Gast GJ, Jonkers PJ, Van Duyl FC, Bak RPM (1999) Bacteria flagellates and nutrients in island fringing coral reef waters: influence of the ocean, the reef and eutrophication. Bulletin of Marine Science 65(2): 523-538

Gauch HG, Gauch Jr HG (1982) Multivariate analysis in community ecology. Cambridge University Press, Cambridge

Gischler E (1997) Cavity dwellers (coelobites) beneath coral rubble in the Florida reef tract. Bulletin of Marine Science 61(2): 467-484 
Gladfelter WB (1982) White-band disease in Acropora palmata: implications for the structure and growth of shallow reefs. Bulletin of Marine Science 32(2): 639-643

Glynn PW (1996) Coral reef bleaching: facts, hypotheses and implications. Global Change Biology 2(6): 495-509

Glynn PW, Morales GEL (1997) Coral reefs of Huatulco, West Mexico: reef development in upwelling Gulf of Tehuantepec. Revista de Biología Tropical 45(3): 1033-1047

Glynn PW, Manzello DP (2015) Bioerosion and coral reef growth: a dynamic balance. In Coral reefs in the Anthropocene. Springer, New York, pp67-97

Goreau TF (1959a) The ecology of Jamaican coral reefs I. Species composition and zonation. Ecology 40(1): 6790

Goreau TF (1959b) The physiology of skeleton formation in corals. I. A method for measuring the rate of calcium deposition by corals under different conditions. The Biological Bulletin 116(1): 59-75

Goreau TF, Goreau NI (1959) The physiology of skeleton formation in corals. II. Calcium deposition by hermatypic corals under various conditions in the reef. The Biological Bulletin 117(2): 239-250

Gorgula SK, Connell SD (2004) Expansive covers of turf-forming algae on human-dominated coast: the relative effects of increasing nutrient and sediment loads. Marine Biology 145(3): 613-619

Govers LL, Lamers LPM, Bouma TJ, De Brouwer JHF, Van Katwijk MM (2014) Eutrophication threatens Caribbean seagrasses-An example from Curaçao and Bonaire. Marine Pollution Bulletin 89(1-2): 481-486

Graham NAJ, Nash KL (2012) The importance of structural complexity in coral reef ecosystems. Coral Reefs 32(2): 315-326

Granados C, Camargo C, Zea S, Sánchez J (2008) Phylogenetic relationships among zooxanthellae (Symbiodinium) associated to excavating sponges (Cliona spp.) reveal an unexpected lineage in the Caribbean. Molecular Phylogenetics and Evolution 49(2): 554-560

Green DH, Edmunds PJ, Carpenter RC (2008) Increasing relative abundance of Porites astreoides on Caribbean reefs mediated by an overall decline in coral cover. Marine Ecology Progress Series 359: 1-10

Grigg RW, Polovina JJ, Atkinson MJ (1984) Model of a coral reef ecosystem. Coral Reefs 3(1): 23-27

Grottoli AG, Warner ME, Levas SJ, Aschaffenburg MD, Schoepf V, McGinley M, Baumann J, Matsui Y (2014) The cumulative impact of annual coral bleaching can turn some coral species winners into losers. Global Change Biology 20(12): 3823-3833

Guzmán JM, Rodríguez J, Martínez J, Contreras JM, González D (2006) The demography of Latin America and the Caribbean since 1950. Population 61(5): 519-620

Haas AF, El-Zibdah M, Wild C (2010) Seasonal monitoring of coral-algae interactions in fringing reefs of the Gulf of Aqaba, Northern Red Sea. Coral Reefs 29(1): 93-103

Haas AF, Fairoz MFM, Kelly LW, Nelson CE, Dinsdale EA, Edwards RA, Giles S, Hatay M, Hisakawa N, Knowles B (2016) Global microbialization of coral reefs. Nature Microbiology 1(6): 16042

Hallock P (2005) Global change and modern coral reefs: new opportunities to understand shallow-water carbonate depositional processes. Sedimentary Geology 175(1-4): 19-33

Harmelin-Vivien M., Harmelin JG, Leboulleux V (1995) Microhabitat requirements for settlement of juvenile sparid fishes on Mediterranean rocky shores. In Space Partition within Aquatic Ecosystems. Springer Dordrecht, pp.309-320

Harris DL, Rovere A, Casella E, Power H, Canavesio R, Collin A, Pomeroy A, Webster JM, Parravicini V (2018) Coral reef structural complexity provides important coastal protection from waves under rising sea levels. Science advances 4(2): eaao4350

Harvell CD, Kim K, Burkholder JM, Colwell RR, Epstein PR, Grimes DJ, Hofmann E, Lipp E, Osterhaus A, Overstreet RM (1999) Emerging marine diseases--climate links and anthropogenic factors. Science 285(5433): 1505-1510

Hawkins JP, Roberts CM, Van'T Hof T, De Meyer K, Tratalos J, Aldam C (1999) Effects of recreational scuba diving on Caribbean coral and fish communities. Conservation Biology 13(4): 888-897

Hearn CJ (1999) Wave-breaking hydrodynamics within coral reef systems and the effect of changing relative sea level. Journal of Geophysical Research: Oceans 104(12): 30007-30019

Hill J, Wilkinson CLIVE (2004) Methods for ecological monitoring of coral reefs. Australian Institute of Marine Science, Townsville 117

Hill M (1996) Symbiotic zooxanthellae enhance boring and growth rates of the tropical sponge Anthosigmella varians forma varians. Marine Biology 125(4): 649-654 
Epilogue

Hoegh-Guldberg O (1999) Climate change, coral bleaching and the future of the world's coral reefs. Marine and Freshwater Research 50(8): 839-866

Hoegh-Guldberg O, Mumby PJ, Hooten AJ, Steneck RS, Greenfield P, Gomez E, Harvell CD, Sale PF, Edwards AJ, Caldeira K (2007) Coral reefs under rapid climate change and ocean acidification. Science 318(5857): 1737-1742

Hoegh-Guldberg O, Bruno JF (2010) The impact of climate change on the world's marine ecosystems. Science 328(5985): 1523-1528

Hoeke RK, McInnes KL, Kruger JC, McNaught RJ, Hunter JR, Smithers SG (2013) Widespread inundation of Pacific islands triggered by distant-source wind-waves. Global and Planetary Change 108: 128-138.

Hoeksema BW, Reimer JD, Vonk R (2017) Biodiversity of Caribbean coral reefs (with a focus on the Dutch Caribbean). Marine Biodiversity 47: 1-10

Holmes G, Ortiz J-C, Schönberg CHL (2009) Bioerosion rates of the sponge Cliona orientalis Thiele, 1900: spatial variation over short distances. Facies 55(2): 203-211

Hopkinson CS, Lugo AE, Alber M, Covich AP, Van Bloem SJ (2008) Forecasting effects of sea-level rise and windstorms on coastal and inland ecosystems. Frontiers in Ecology and the Environment 6(5): 255-263

Hudson J (1977) Long-term bioerosion rates on a Florida reef: a new method. Proceedings of the 3rd International Coral Reef Symposium 2: 491-497

Hughes TP (1994) Catastrophes, phase shifts, and large-scale degradation of a Caribbean coral reef. Science 265(5178): 1547-1551

Hughes TP, Szmant A, Steneck RS, Carpenter RC, Miller SL (1999) Algal blooms on coral reefs: what are the causes? Limnology and Oceanography 44(6): 1583-1586

Hughes TP, Tanner JE (2000) Recruitment failure, life histories, and long-term decline of Caribbean corals. Ecology 81(8): 2250-2263

Hughes TP, Baird AH, Bellwood DR, Card M, Connolly SR, Folke C, Grosberg R, Hoegh-Guldberg O, Jackson JBC, Kleypas J, Lough JM, Marshall P, Nystöm M, Palumbi SR, Pandolfi JM, Rosen B, Roughgarden J (2003) Climate change, human impacts, and the resilience of coral reefs. Science 301(5635): 929-933

Hughes TP, Rodrigues MJ, Bellwood DR, Ceccarelli D, Hoegh-Guldberg O, McCook L, Moltschaniwskyj N, Pratchett MS, Steneck RS, Willis B (2007) Phase shifts, herbivory, and the resilience of coral reefs to climate change. Current Biology 17(4): 360-365

Hughes TP, Barnes ML, Bellwood DR, Cinner JE, Cumming GS, Jackson JBC, Kleypas J, Van De Leemput IA, Lough JM, Morrison TH, Palumbi SR, Van Nes EH, Scheffer M (2017) Coral reefs in the Anthropocene. Nature 546(7656): 82-90

Huisman J, Codd GA, Paerl HW, Ibelings BW, Verspagen JM, Visser PM (2018) Cyanobacterial blooms. Nature Reviews Microbiology 16(8): 471-483

Hutchings PA (1986) Biological destruction of coral reefs. Coral reefs 4(4): 239-252

Hydes D, Aoyama M, Aminot A, Bakker K, Becker S, Coverly S, Daniel A, Dickson A, Grosso O, Kerouel R (2010) Determination of dissolved nutrients (N, P, Si) in seawater with high precision and intercomparability using gas-segmented continuous flow analysers. In The GOSHIP repeat hydrography manual: a collection of expert report and guidelines, IOCCP report number 14, ICPO publication series number 134, UNESCO-IOC, Paris, France.

Jackson JBC, Jung P, Coates AG, Collins LS (1993) Diversity and extinction of tropical American mollusks and emergence of the Isthmus of Panama. Science 260(5114): 1624-1626

Jackson JBC (1997) Reefs since Columbus. Coral reefs 16(1): 23-32

Jackson JBC (2001) What was natural in the coastal oceans? Procedings of the Royal Society B 98(10): 5411-5418

Jackson JBC, Kirby MX, Berger WH, Bjorndal KA, Botsford LW, Bourque BJ, Bradbury RH, Cooke R, Erlandson J, Estes JA (2001) Historical overfishing and the recent collapse of coastal ecosystems. Science 293(5530): 629-637

Jackson JBC, Donovan M, Cramer K, Lam V (eds) (2014) Status and trends of Caribbean coral reefs: 1970-2012. Global Coral Reef Monitoring Network, International Union for the Conservation of Nature Global Marine and Polar Program, Washington, DC

Jacques T, Pilson M (1980) Experimental ecology of the temperate scleractinian coral Astrangia danae I. Partition of respiration, photosynthesis and calcification between host and symbionts. Marine Biology 60(2-3): 167-178 
Januchowski-Hartley FA, Graham NA, Wilson SK, Jennings S, Perry CT (2017) Drivers and predictions of coral reef carbonate budget trajectories. Proceedings of the Royal Society of London B: Biological Sciences 284(1847): 20162533

Johnson AE, Jackson JB (2015) Fisher and diver perceptions of coral reef degradation and implications for sustainable management. Global Ecology and Conservation 3: 890-899

Kendall ME (1948) Rank correlation methods 4th ed. Griffin, London

Kennedy EV, Perry CT, Halloran PR, Iglesias-Prieto R, Schönberg CHL, Wisshak M, Form AU, CarricartGanivet JP, Fine M, Eakin CM (2013) Avoiding coral reef functional collapse requires local and global action. Current Biology 23(10): 912-918

Kim YJ, Gu C (2004) Smoothing spline Gaussian regression: more scalable computation via efficient approximation. Journal of the Royal Statistical Society: Series B (Statistical Methodology) 66(2): 337-356

Kleypas JA, Buddemeier RW, Gattuso J-P (2001) The future of coral reefs in an age of global change. International Journal of Earth Sciences 90:426-437

Knowlton N (2001) The future of coral reefs. Procedings of the National Academy of Sciences 98(10): 5419-5425

Knowlton N, Jackson JBC (2008) Shifting baselines, local impacts, and global change on coral reefs. PLoS Biology 6(2): e54

Kohler KE, Gill SM (2006) Coral Point Count with Excel extensions (CPCe): A Visual Basic program for the determination of coral and substrate coverage using random point count methodology. Computers and Geosciences 32(9): 1259-1269

Komsta L, Novomestky F (2015) Moments, cumulants, skewness, kurtosis and related tests. $R$ package version 0.14

Koop K, Booth D, Broadbent A, Brodie J, Bucher D, Capone D, Coll J, Dennison W, Erdmann M, Harrison P (2001) ENCORE: the effect of nutrient enrichment on coral reefs. Synthesis of results and conclusions. Marine Pollution Bulletin 42(2): 91-120

Kramer PA (2003) Synthesis of coral reef health indicators for the western Atlantic: results of the AGRRA program (1997-2000). Atoll Research Bulletin 496: 1-57

Kruskal JB (1964) Multidimensional scaling by optimizing goodness of fit to a nonmetric hypothesis. Psychometrika 29(1): 1-27

Kuffner IB, Paul VJ (2001) Effects of nitrate, phosphate and iron on the growth of macroalgae and benthic cyanobacteria from Cocos Lagoon, Guam. Marine Ecology Progress Series 222: 63-72

Kuffner IB, Andersson AJ, Jokiel PL, Rodgers KS, Mackenzie FT (2008) Decreased abundance of crustose coralline algae due to ocean acidification. Nature Geoscience 1(2): 114-117

Kuffner IB, Walters LJ, Becerro MA, Paul VJ, Ritson-Williams R, Beach KS (2006) Inhibition of coral recruitment by macroalgae and cyanobacteria. Marine Ecology Progress Series 323: 107-117

Lamb JB, True JD, Piromvaragorn S, Willis BL (2014) Scuba diving damage and intensity of tourist activities increases coral disease prevalence. Biological Conservation 178: 88-96

Lapointe BE, Mallin MA (2011) Nutrient enrichment and eutrophication on fringing coral reefs of Bonaire and Curaçao, Netherlands Antilles. Report to the United Nations Environment Programme for the NACRI Coral Reef Monitoring Program, Harbor Branch Oceanographic Institute, Ft Pierce, Florida

Larkum AWD, Kennedy IR, Muller WJ (1988) Nitrogen fixation on a coral reef. Marine Biology 98(1): 143-155

Legendre P, Oden NL, Sokal RR, Vaudor A, Kim J (1990) Approximate analysis of variance of spatially autocorrelated regional data. Journal of Classification 7 (1): 53-75

Legendre P, Legendre L (2012) Numerical ecology: Third English edition. Developments in environmental modelling 20. Elsevier, Amsterdam, the Netherlands.

Leigh EG, Paine RT, Quinn JF, Suchanek TH (1987) Wave energy and intertidal productivity. Proceedings of the National Academy of Sciences 84(5): 1314-1318

Lewis SM (1986) The role of herbivorous fishes in the organization of a Caribbean reef community. Ecological monographs 56(3): 183-200

Littler MM, Littler DS (1998) An undescribed fungal pathogen of reef-forming crustose corraline algae discovered in American Samoa. Coral Reefs 17(2): 144-144

Liu X, Byrne RH, Lindemuth M, Easley R, Mathis JT (2015) An automated procedure for laboratory and shipboard spectrophotometric measurements of seawater alkalinity: Continuously monitored single-step acid additions. Marine Chemistry 174: 141-146 
Loh TL, McMurray SE, Henkel TP, Vicente J, Pawlik JR (2015) Indirect effects of overfishing on Caribbean reefs: sponges overgrow reef-building corals. PeerJ 3: e901

Luckhurst BE, Luckhurst K (1978) Analysis of the influence of substrate variables on coral reef fish communities. Marine Biology 49(4): 317-323

Lugo AE, Rogers CS, Nixon SW (2000) Hurricanes, coral reefs and rainforests: resistance, ruin and recovery in the Caribbean. AMBIO: A Journal of the Human Environment 29(2): 106-114

MacArthur RH, MacArthur JW (1961) On bird species diversity. Ecology 42(3): 594-598

MacGeachy JK, Stearn CW (1976) Boring by macro-organisms in the coral Montastrea annularis on Barbados Reefs. International Review of Hydrobiology 61(6): 715-745

Maechler M (2018) Finding Groups in Data": Cluster Analysis Extended Rousseeuw et. Documentation for software package The Comprehensive R Archive Network (CRAN): Wien

Mardia KV (1970) Measures of multivariate skewness and kurtosis with applications. Biometrika 57(3): 519-530

Marlow J, Schönberg CHL, Davy SK, Haris A, Jompa J, Bell JJ (2018) Bioeroding sponge assemblages: the importance of substrate availability and sediment. Journal of the Marine Biological Association of the United Kingdom 1-16

McAllister DE (1991) What is the status of the world's coral reef fishes. Sea Wind 5(1): 14-18

McClanahan TR, Graham NAJ, Darling ES (2014) Coral reefs in a crystal ball: predicting the future from the vulnerability of corals and reef fishes to multiple stressors. Current Opinion in Enviromental Sustainability 7: 59-64

McCook L (2001) Competition between corals and algal turfs along a gradient of terrestrial influence in the nearshore central Great Barrier Reef. Coral Reefs 19(4): 419-425

McCook L, Jompa J, Diaz-Pulido G (2001) Competition between corals and algae on coral reefs: a review of evidence and mechanisms. Coral Reefs 19(4): 400-417

McMurray SE, Finelli CM, Pawlik JR (2015) Population dynamics of giant barrel sponges on Florida coral reefs. Journal of Experimental Marine Biology and Ecology 473: 73-80

Meesters E, Knijn R, Willemsen P, Pennartz R, Roebers G, van Soest RV (1991) Sub-rubble communities of Curaçao and Bonaire coral reefs. Coral Reefs 10(4): 189-197

Meesters EH, Bos A, Gast GJ (1992) Effects of sedimentation and lesion position on coral tissue regeneration. Proceedings of the 7th International Coral Reef Symposium 2: 681-688

Meesters EH, Wesseling I, Bak RPM (1996) Partial mortality in three species of reef-building corals and the relation with colony morphology. Bulletin of Marine Science 58(3): 838-852

Meesters EH, Pauchli W, Bak RPM (1997a) Predicting regeneration of physical damage on a reef-building coral by regeneration capacity and lesion shape. Marine Ecology Progress Series 146: 91-99

Meesters EH, Wesseling I, Bak RPM (1997b) Coral colony tissue damage in six species of reef-building corals: partial mortality in relation with depth and surface area. Netherlands Journal of Sea Research 37(1-2): 131-144

Moberg F, Rönnbäck P (2003) Ecosystem services of the tropical seascape: interactions, substitutions and restoration. Ocean \& Coast Manage 46(1-2): 27-46

Monismith SG, Rogers JS, Koweek D, Dunbar RB (2015) Frictional wave dissipation on a remarkably rough reef. Geophysical Research Letters 42(10): 4063-4071

Mücher S, Suomalainen J, Stuiver J, Meesters EH (2017) Hyperspectral Coral Reef Classification of Bonaire (No. C062/17). Retrieved from https://library.wur.nl/WebQuery/wurpubs/fulltext/422722

Mueller B, de Goeij JM, Vermeij MJA, Mulders Y, van der Ent E, Ribes M, van Duyl FC (2014) Natural diet of coral-excavating sponges consists mainly of dissolved organic carbon (DOC). PloS One 9: e90152

Mueller B (2017) First documentation of encrusting specimen of Cliona delitrix on Curaçao: a cause for concern? Marine Biodiversity Records 10(1): 1-4

Mumby PJ, Dahlgren CP, Harborne AR, Kappel CV, Micheli F, Brumbaugh DR, Holmes KE, Mendes JM, Broad K, Sanchirico JN (2006) Fishing, trophic cascades, and the process of grazing on coral reefs. Science 311:98-101

Mumby PJ, Harborne AR, Williams J, Kappel CV, Brumbaugh DR, Micheli F, Holmes KE, Dahlgren CP, Paris CB, Blackwell PG (2007) Trophic cascade facilitates coral recruitment in a marine reserve. Proceedings of the National Academy of Sciences 104(20): 8362-8367 
Mumby PJ, Broad K, Brumbaugh DR, Dahlgren CP, Harborne AR, Hastings A, Holmes KE, Kappel CV, Micheli F, Sanchirico JN (2008) Coral reef habitats as surrogates of species, ecological functions, and ecosystem services. Conservation Biology 22(4): 941-951

Murdoch TJT (2007) A functional group approach for predicting the composition of hard coral assemblages in Florida and Bermuda. PhD thesis, University of South Alabama, Mobile

Murphy G, Perry C, Chin P, McCoy C (2016) New approaches to quantifying bioerosion by endolithic sponge populations: applications to the coral reefs of Grand Cayman. Coral Reefs 35(3): 1109-1121

Nagelkerken I, Bouma S, Van Den Akker S, Bak RPM (2000) Growth and survival of unattached Madracis mirabilis fragments transplanted to different reef sites, and the implication for reef rehabilitation. Bulletin of Marine Science 66(2): 497-505

Nagelkerken I, Vermonden K, Moraes OCC, Debrot AO, Nagelkerken WP (2005) Changes in coral reef communities and an associated reef fish species, Cephalopholis cruentata (Lacepede), after 30 years on Curaçao (Netherlands Antilles). Hydrobiologia 549(1): 145-154

Nagle DG, Paul VJ (1998) Chemical defense of a marine cyanobacterial bloom. Journal of Experimental Marine Biology and Ecology 225(1): 29-38

Nava H, Carballo JL (2008) Chemical and mechanical bioerosion of boring sponges from Mexican Pacific coral reefs. Journal of experimental Biology 211(17): 2827-2831

Neumann AC (1966) Observations on coastal erosion in Bermuda and measurements of the boring rate of the sponge, Cliona lampa. Limnology and Oceanography 11(1): 92-108

Newell ND (1971) An outline history of tropical organic reefs. American Museum Novitates 2465: 1-37

Newman MJ, Paredes GA, Sala E, Jackson JBC (2006) Structure of Caribbean coral reef communities across a large gradient of fish biomass. Ecology letters 9(11): 1216-1227

Newman SP, Meesters EH, Dryden CS, Williams SM, Sanchez C, Mumby PJ, Polunin NVC (2015) Reef flattening effects on total richness and species responses in the Caribbean. Journal of Animal Ecology 84(6): 1678-1689

Nugues MM, Roberts CM (2003a) Coral mortality and interaction with algae in relation to sedimentation. Coral reefs 22(4): 507-516

Nugues MM, Roberts CM (2003b) Partial mortality in massive reef corals as an indicator of sediment stress on coral reefs. Marine Pollution Bulletin 46(3): 314-323

Nugues MM, Bak RPM (2008) Long-term dynamics of the brown macroalga Lobophora variegata on deep reefs in Curaçao. Coral Reefs 27(2): 389-393

Nyström M (2006) Redundancy and response diversity of functional groups: implications for the resilience of coral reefs. AMBIO: A Journal of the Human Environment 35(1): 30-35

Nyström M, Folke C, Moberg F (2000) Coral reef disturbance and resilience in a human-dominated environment. Trends in Ecology \& Evolution 15(10): 413-417

O'Dea A, Shaffer ML, Doughty DR, Wake TA, Rodriguez FA (2014) Evidence of size-selective evolution in the fighting conch from prehistoric subsistence harvesting. Proceedings of the Royal Society of London B: Biological Sciences 281:20140159

Odum HT, Odum EP (1955) Trophic structure and productivity of a windward coral reef community on Eniwetok Atoll. Ecological Monographs 25(3): 291-320

Ogden JC (1977) Carbonate-sediment production by parrot fish and sea urchins on Caribbean reefs. In Reefs and related carbonates - ecology and sedimentology. Studies in Geology No. 4. American Association of Petroleum Geologists, Tulsa, pp281-288

Oksanen J, Blanchet FG, Kindt R, Legendre P, Minchin PR, O'Hara RB, Simpson GL, Solymos P, Stevens MHH, Wagner H (2013) vegan: Community Ecology Package. R package version 2.3-5.

Orr JC, Fabry VJ, Aumont O, Bopp L, Doney SC, Feely RA, Gnanadesikan A, Gruber N, Ishida A, Joos F (2005) Anthropogenic ocean acidification over the twenty-first century and its impact on calcifying organisms. Nature 437(7059): 681

Paddack, MJ, Reynolds, JD, Aguilar, C, Appeldoorn, RS, Beets, J, Burkett, EW, Chittaro, PM, Clarke, K, Esteves, R, Fonseca, AC, Forrester, GE, Friedlander, AM, García-Sais, J, González-Sansón, G, Jordan, LKB, McClellan, DB, Miller, MW, Molloy, PP, Mumby, PJ, Nagelkerken, I, Nemeth, M, NavasCamacho, R, Pitt, J, Polunin, NVC, Reyes-Nivia, MC, Robertson, DR, Rodríguez-Ramírez, A, Salas, E, Smith, SR, Spieler, RE, Steele, MA, Williams, ID, Wormald, CL, Watkinson, AR, Côté, IM (2009) Recent region-wide declines in Caribbean reef fish abundance. Current Biology 19(7): 590-595 
Paerl HW, Huisman J (2008) Blooms like it hot. Science 320(5872): 57-58

Pandolfi JM, Bradbury RH, Sala E, Hughes TP, Bjorndal KA, Cooke RG, McArdle D, McClenachan L, Newman MJ, Paredes G (2003) Global trajectories of the long-term decline of coral reef ecosystems. Science 301(5635): 955-958

Pandolfi JM, Jackson JBC (2006) Ecological persistence interrupted in Caribbean coral reefs. Ecology Letters 9(7): 818-826

Pang RK (1973) The ecology of some Jamaican excavating sponges. Bulletin of Marine Science 23(2): 227-243

Paul VJ, Thacker RW, Banks K, Golubic S (2005) Benthic cyanobacterial bloom impacts the reefs of South Florida (Broward County, USA). Coral Reefs 24:693-697

Paul VJ (2008) Global warming and cyanobacterial harmful algal blooms Cyanobacterial Harmful Algal Blooms: State of the Science and Research Needs. Adv Exp Med Biol, pp239-257

Pauly D (1995) Anecdotes and the shifting baseline syndrome of fisheries. Trends in Ecology \& Evolution 10(10): 430

Pawlik JR, Burkepile DE, Vega-Thurber RL (2016) A Vicious Circle? Altered Carbon and Nutrient Cycling May Explain the Low Resilience of Caribbean Coral Reefs. BioScience 66(6): 470-476

Pawlik JR, Steindler L, Henkel TP, Beer S, Ilan M (2007) Chemical warfare on coral reefs: Sponge metabolites differentially affect coral symbiosis in situ. Limnology and Oceanography 52(2): 907-911

Perry CT, Spencer T, Kench PS (2008) Carbonate budgets and reef production states: a geomorphic perspective on the ecological phase-shift concept. Coral Reefs 27(4): 853-866

Perry CT, Edinger EN, Kench PS, Murphy GN, Smithers SG, Steneck RS, Mumby PJ (2012) Estimating rates of biologically driven coral reef framework production and erosion: a new census-based carbonate budget methodology and applications to the reefs of Bonaire. Coral Reefs 31(3): 853-868

Perry CT, Murphy GN, Kench PS, Smithers SG, Edinger EN, Steneck RS, Mumby PJ (2013) Caribbean-wide decline in carbonate production threatens coral reef growth. Nature communications 4: 1402

Perry CT, Murphy GN, Kench PS, Edinger EN, Smithers SG, Steneck RS, Mumby PJ (2014) Changing dynamics of Caribbean reef carbonate budgets: emergence of reef bioeroders as critical controls on present and future reef growth potential. Proceeding of the Royal Society B 281(1796): 20142018.

Perry CT, Steneck RS, Murphy GN, Kench PS, Edinger EN, Smithers SG, Mumby PJ (2015a) Regional-scale dominance of non-framework building corals on Caribbean reefs affects carbonate production and future reef growth. Global Change Biology 21(3): 1153-1164

Perry CT, Murphy GN, Graham NA, Wilson SK, Januchowski-Hartley FA, East HK (2015b). Remote coral reefs can sustain high growth potential and may match future sea-level trends. Scientific Reports 5: 18289

Perry CT, Alvarez-Filip L, Graham NAJ, Mumby PJ, Wilson SK, Kench PS, Manzellp PD, Morgan KM, Slangen ABA, Thomson PD, Januchowski-Hartley F, Smithers SG, Steneck RS, Carlton Rm Edinger EN, Enochs IC, Estrada-Saldivar N, Haywood MDE, Kolodziej G, Perez-Cervantes E, Suchley A, Valentino L, Boenish R, Wilson M, Macdonald C (2018) Loss of coral reef growth capacity to track future increases in sea level. Nature 558(7710): 396-400

Pielou EC (1969) An introduction to mathematical ecology. Wiley-Interscience, New-York.

Pomponi SA (1980) Cytological mechanisms of calcium carbonate excavation by boring sponges. International review of Cytology 65: 301-319

Pratchett MS, Hoey AS, Wilson SK, Messmer V. Graham NA (2011) Changes in biodiversity and functioning of reef fish assemblages following coral bleaching and coral loss. Diversity, 3(3): 424-452

Quataert E, Storlazzi C, Rooijen A, Cheriton O, Dongeren A (2015) The influence of coral reefs and climate change on wave-driven flooding of tropical coastlines. Geophysical Research Letters 42(15): 6407-6415

R Core Team (2002) The R stats package. R Foundation for Statistical Computing, Vienna, Austria: Available from: http://www R-project org

R Core Team (2013) R: A language and environment for statistical computing. R Foundation for Statistical Computing, Vienna, Austria. 2013. ISBN 3-900051-07-0

Reaka-Kudla ML (1997) The global biodiversity of coral reefs: a comparison with rain forests. Biodiversity II: Understanding and protecting our biological resources 2:551

Renken H, Mumby PJ, Matsikis I, Edwards HJ (2010) Effects of physical environmental conditions on the patch dynamics of Dictyota pulchella and Lobophora variegata on Caribbean coral reefs. Marine Ecology Progress Series 403:63-74

Risk MJ (1972) Fish diversity on a coral reef in the Virgin Islands. Atoll Research Bulletin 153: 1-6 
Epilogue

Ritson-Williams R, Paul VJ, Bonito V (2005) Marine benthic cyanobacteria overgrow coral reef organisms. Coral Reefs 24:629-629

Rix L, Goeij JM, Oevelen D, Struck U, Al-Horani FA, Wild C, Naumann MS (2017) Differential recycling of coral and algal dissolved organic matter via the sponge loop. Functional Ecology 31(4): 778-789

Rix L, De Goeij JM, Mueller CE, Struck U, Middelburg JJ, Van Duyl FC, Al-Horani FA, Wild C, Naumann MS, Van Oevelen D (2016) Coral mucus fuels the sponge loop in warm-and cold-water coral reef ecosystems. Scientific Reports 6: 18715

Roberts DW, Roberts MDW (2016) Package 'labdsv'. Ordination and Multivariate analysis for ecology. R package version 1(1)

Roff G, Mumby PJ (2012) Global disparity in the resilience of coral reefs. Trends in ecology \& evolution 27(7): 404-413

Roff G, Chollett I, Doropoulos C, Golbuu Y, Steneck RS, Isechal AL, van Woesik R, Mumby PJ (2015) Exposure-driven macroalgal phase shift following catastrophic disturbance on coral reefs. Coral Reefs 34(3): 715-725

Rogers CS (1990) Responses of coral reefs and reef organisms to sedimentation. Marine Ecology Progress Series 62(1): 185-202

Rose CS, Risk MJ (1985) Increase in Cliona delitrix infestation of Montastrea cavernosa heads on an organically polluted portion of the Grand Cayman fringing reef. Marine Ecology 6(4): 345-363

Rützler K (1974) The burrowing sponges of Bermuda. Smithsonian Institution Press, Washington

Rützler K (1975) The role of burrowing sponges in bioerosion. Oecologia 19(3): 203-216

Rützler K (2002) Impact of crustose clionid sponges on Caribbean reef corals. Acta Geologica Hispanica 37(1): 61-72

Rützler K, Rieger G (1973) Sponge burrowing: fine structure of Cliona lampa penetrating calcareous substrata. Marine Biology 21(2): 144-162

Sammarco PW, Levinton JS, Ogden JC (1974) Grazing and control of coral-reef community structure by Diadema antillarum philippi (Echinodermata-Echinoidea) - preliminary study. Journal of Marine Research 32(1): 47-53

Sammarco PW (1982) Effects of grazing by Diadema antillarum Philippi (Echinodermata: Echinoidea) on algal diversity and community structure. Journal of Experimental Marine Biology and Ecology 65(1): 83-105

Sandin SA, Sampayo EM, Vermeij MJA (2008a) Coral reef fish and benthic community structure of Bonaire and Curaçao, Netherlands Antilles. Caribbean Journal of Science 44(2): 137-144

Sandin SA, Smith JE, DeMartini EE, Dinsdale EA, Donner SD, Friedlander AM, Konotchick T, Malay M, Maragos JE, Obura D (2008b) Baselines and degradation of coral reefs in the northern Line Islands. PloS One 3(2): e1548

Scatterday JW (1974) Reefs and associated coral assemblages off Bonaire, Netherlands Antilles, and their bearing on Pleistocene and recent reef models. Proceedings of the 2nd International Coral Reef Symposium 2: 85-106

Schönberg CHL (2002a) Sponges of the 'Cliona viridis complex'-a key for species identification. Proceedings of 9th international coral reef symposium 1: 295-299

Schönberg CHL (2002b) Substrate effects on the bioeroding demosponge Cliona orientalis. 1. Bioerosion rates. Marine Ecology 23(4): 313-326

Schönberg CHL (2006) Growth and erosion of the zooxanthellate Australian bioeroding sponge Cliona orientalis are enhanced in light. Proceedings of the 10th International Coral Reef Symposium 1: 168-174

Schönberg CHL (2008) A history of sponge erosion: from past myths and hypotheses to recent approaches. In Current developments in bioerosion. Springer, Springer, pp165-202

Schönberg CHL, Fang JK-H, Carballo JL (2017a) Bioeroding sponges and the future of coral reefs. In Climate Change, Ocean Acidification and Sponges. Springer, pp179-372

Schönberg CHL, Fang JK, Carreiro-Silva M, Tribollet A, Wisshak M (2017b) Bioerosion: the other ocean acidification problem. ICES Journal of Marine Science 74(4): 895-925

Scoffin TP (1980) Calcium carbonate budget of a fringing reef on the west coast of Barbados. Part II-Erosion, sediments and internal structure. Bulletin of Marine Science 30: 475-508

Shannon CE, Weaver (1949): The Mathematical Theory of Communication. University of Illinois Press, Urbana

Sheehan PM (1985) Reefs are not so different - they follow the evolutionary pattern of level-bottom communities. Geology 13(2): 46-49 
Sheehan PM (1996) A new look at ecologic evolutionary units (EEUs). Palaeogeography, Palaeoclimatology, Palaeoecology 127(1-4): 21-32

Sheppard CR, Spalding M, Bradshaw C, Wilson S (2002) Erosion vs. recovery of coral reefs after 1998 El Niño: Chagos reefs, Indian Ocean. AMBIO: A Journal of the Human Environment 31(1): 40-48

Sheppard, CR, Dixon DJ, Gourlay M, Sheppard A, Payet R (2005) Coral mortality increases wave energy reaching shores protected by reef flats: examples from the Seychelles. Estuarine, Coastal and Shelf Science 64(2-3): 223-234

Silbiger NJ, Nelson CE, Remple K, Sevilla JK, Quinlan ZA, Putnam HM, Fox D, Donahue MJ (2018) Nutrient pollution disrupts key ecosystem functions on coral reefs. Proceedings of the Royal Society B 285(1880): 20172718

Simpson EH (1949) Measurement of diversity. Nature 163: 688

Siung-Chang A (1997) A review of marine pollution issues in the Caribbean. Environmental Geochemistry and Health 19(2):45-55

Slangen ABA, Carson M, Katsman CA, Van de Wal RSW, Köhl A, Vermeersen LLA, Stammer D (2014) Projecting twenty-first century regional sea-level changes. Climatic Change 124(1-2): 317-332

Slijkerman DME, De León R, De Vries P (2014) A baseline water quality assessment of the coastal reefs of Bonaire, Southern Caribbean. Marine Pollution Bulletin 86(1-2): 523-529

Smith JE, Price NN, Nelson CE, Haas AF (2013) Coupled changes in oxygen concentration and pH caused by metabolism of benthic coral reef organisms. Marine Biology 160(9): 2437-2447

Smith JE, Brainard R, Carter A, Grillo S, Edwards C, Harris J, Lewis L, Obura D, Rohwer F, Sala E (2016) Re-evaluating the health of coral reef communities: baselines and evidence for human impacts across the central Pacific. Proceedings of Royal Society B 283(1822): 20151985

Smith S, Kinsey D (1976) Calcium carbonate production, coral reef growth, and sea level change. Science 194(4268): 937-939

Smith VH, Schindler DW (2009) Eutrophication science: where do we go from here? Trends in Ecology \& Evolution 24(4): 201-207

Sokal RR, Rohlf FJ (1962) The comparison of dendrograms by objective methods. JSTOR Taxon 11(2): 33-40

Sommer B, Harrison PL, Scheffers SR (2010) Aggressive colonial ascidian impacting deep coral reefs at Bonaire, Netherlands Antilles. Coral Reefs 29(1): 245-245

Sommer B, Harrison PL, Brooks L, Scheffers SR (2011) Coral community decline at Bonaire, southern Caribbean. Bulletin of Marine Science 87(3): 541-565

Spalding M, Grenfell A (1997) New estimates of global and regional coral reef areas. Coral Reefs 16(4): 225-230

Spalding M, Spalding MD, Ravilious C, Green EP (2001) World atlas of coral reefs. University of California Press, California

Stearn CW, Scoffin TP, Martindale W (1977) Calcium Carbonate Budget of a Fringing Reef on the West Coast of Barbados Part I - Zonation and Productivity. Bulletin of Marine Science 27(3): 479-510

Steneck RS, Arnold SN, Rasher DB (2013) Status and trends of Bonaire's reefs in 2013: Causes for optimism. University of Maine, School of Marine Sciences, 124

Stokes MD, Leichter JJ, Genovese SJ (2010) Long-term declines in coral cover at Bonaire, Netherlands Antilles. Atoll Research Bulletin 582: 1-23

Storlazzi CD, Gingerich SB, van Dongeren A, Cheriton OM, Swarzenski PW, Quataert E, Voss CI, Field DW, Annamalai H, Piniak GA, McCall R (2018) Most atolls will be uninhabitable by the mid-21st century because of sea-level rise exacerbating wave-driven flooding. Science Advances 4(4): eaap9741

Terry JP, Falkland AC (2010) Responses of atoll freshwater lenses to storm-surge overwash in the Northern Cook Islands. Hydrogeology Journal 18(3): 749-759

Thacker RW, Nagle DG, Paul VJ (1997) Effects of repeated exposures to marine cyanobacterial secondary metabolites on feeding by juvenile rabbitfish and parrotfish. Marine Ecology Progress Series 147: 21-29

Vacelet J, Boury-Esnault N (1995) Carnivorous sponges. Nature 373(6512): 333

Van Duyl FC, Bak RPM, Sybesma J (1981) The ecology of the tropical compound ascidian Trididemnum solidum. I. Reproductive strategy and larval behaviour. Marine Ecology Progress Series 6(1): 35-42

Van Duyl FC (1985) Atlas of the Living Reefs of Curaçao and Bonaire (Netherlands Antilles). Foundation for Scientific Research in Surinam and the Netherlands Antilles 117: Utrecht, The Netherlands

Van Woesik R, Sakai K, Ganase A, Loya Y (2011) Revisiting the winners and the losers a decade after coral bleaching. Marine Ecology Progress Series 434: 67-76 
Epilogue

Van Woesik R, Franklin EC, O'Leary J, McClanahan TR, Klaus JS, Budd AF (2012) Hosts of the PlioPleistocene past reflect modern-day coral vulnerability. Proceedings of the Royal Society B 279(1737): 2448 $-2456$

Van Soest RWM. Cliona delitrix Pang, 1973. In: Van Soest RWM, Boury-Esnault N, Hooper JNA, Rützler K, de Voogd NJ, Alvarez de Glasby B, Hajdu E, Pisera AB, Manconi R, Schönberg CHL, Klautau M, Picton B, Kelly M, Vacelet J, Dohrmann M, Díaz M-C, Cárdenas P, Carballo JL. World Porifera database; 2010. Accessed 12 Mar 2017

Vecsei A (2001) Fore-reef carbonate production: development of a regional census-based method and first estimates. Palaeogeography, Palaeoclimatology, Palaeoecology 175(1-4): 185-200

Vega-Thurber RL, Willner-Hall D, Rodriguez-Mueller B, Desnues C, Edwards RA, Angly F, Dinsdale E, Kelly L, Rohwer F (2009) Metagenomic analysis of stressed coral holobionts. Environental Microbiology 11(8): 21482163

Vega-Thurber RL, Burkepile DE, Fuchs C, Shantz AA, McMinds R, Zaneveld JR (2014) Chronic nutrient enrichment increases prevalence and severity of coral disease and bleaching. Global Change Biology 20(2): 544-554

Vermeij MJA, Sandin SA (2008) Density-dependent settlement and mortality structure the earliest life phases of a coral population. Ecology 89(7): 1994-2004

Vermeij MJA, Van Moorselaar I, Engelhard S, Hörnlein C, Vonk SM, Visser PM (2010) The effects of nutrient enrichment and herbivore abundance on the ability of turf algae to overgrow coral in the Caribbean. PLoS One 5(12): e14312

Vermeij MJA, Bakker J, van der Hal N, Bak RPM (2011) Juvenile coral abundance has decreased by more than $50 \%$ in only three decades on a small Caribbean island. Diversity 3(3): 296-307

Veron JEN (2014) Results of an update of the Corals of the World Information Base for the Listing Determination of 66 Coral Species under the Endangered Species Act. Report to the Western Pacific Regional Fishery Management Council, Honolulu

Vitousek S, Barnard PL, Fletcher CH, Frazer N, Erikson L, Storlazzi CD (2017) Doubling of coastal flooding frequency within decades due to sea-level rise. Scientific Reports 7(1): 1399

Ward-Paige CS, Risk MJ, Sherwood OA, Jaap WC (2005) Clionid sponge surveys on the Florida Reef Tract suggest land-based nutrient inputs. Marine Pollution Bulletin 51(5-7): 570-579

Ward Jr JH (1963) Hierarchical grouping to optimize an objective function. Journal of the American Statistical Association 58(301): 236-244

Webb AE, van Heuven SMAC, de Bakker DM, van Duyl FC, Reichart G-J, de Nooijer LJ (2017) Combined Effects of Experimental Acidification and Eutrophication on Reef Sponge Bioerosion Rates. Frontiers in Marine Science 4: 311

Weisz JB, Massaro AJ, Ramsby BD, Hill MS (2010) Zooxanthellar symbionts shape host sponge trophic status through translocation of carbon. The Biological Bulletin 219(3): 189-197

Wilkinson, C.R. (ed.) (2004) Status of Coral Reefs of the World: 2004. Volumes 1 and 2. Australian Institute of Marine Science, Townsville, Australia

Wilkinson CR, Souter DN (2008) Status of Caribbean coral reefs after bleaching and hurricanes in 2005. Global Coral Reef Monitoring Network, and Reef and Rainforest Research Centre, Townsville, Australia

Wilks DS (2011) Cluster analysis. In International geophysics 100: pp603-616, Academic press

Williams DMB, Roth CH, Reichelt R, Ridd P, Rayment GE, Larcombe P, Brodie J, Pearson R, Wilkinson C, Talbot F (2002) The current level of scientific understanding on impacts of terrestrial run-off on the Great Barrier Reef World Heritage Area. CRC Reef Research Centre, Townsville, Australia

Wisshak M, Schönberg CHL, Form AU, Freiwald A (2012) Ocean acidification accelerates reef bioerosion. PloS One 7(9): e45124

Wisshak M, Schönberg CHL, Form AU, Freiwald A (2013) Effects of ocean acidification and global warming on reef bioerosion - lessons from a Clionaid sponge. Aquatic Biology 19(2): 111-127

Wisshak M, Schönberg CHL, Form AU, Freiwald A (2014) Sponge bioerosion accelerated by ocean acidification across species and latitudes? Helgoland Marine Research 68(2): 253

Wood S (2012) mgcv: Mixed GAM Computation Vehicle with GCV/AIC/REML Smoothness Estimation. R package Version 1.7-17. URL https://cran r-project org/web/packages/mgcv/index html

Woodring W (1954) Caribbean land and sea through the ages. Geological Society of America Bulletin 65(8): 719732 
Wooldridge SA (2009) Water quality and coral bleaching thresholds: Formalising the linkage for the inshore reefs of the Great Barrier Reef, Australia. Marine Pollution Bulletin 58(5): 745-751

Yao W, Byrne RH (1998) Simplified seawater alkalinity analysis: use of linear array spectrometers. Deep Sea Research Part I: Oceanographic Research Papers 45(8): 1383-1392

Yates KK, Zawada DG, Smiley NA, Tiling-Range G (2017) Divergence of seafloor elevation and sea level rise in coral reef ecosystems. Biogeosciences 14(6): 1739

Zaneveld JR, Burkepile DE, Shantz AA, Pritchard CE, McMinds R, Payet JP, Welsh R, Correa AMS, Lemoine NP, Rosales S (2016) Overfishing and nutrient pollution interact with temperature to disrupt coral reefs down to microbial scales. Nature Communications 7: 11833

Zea S, Weil E (2003) Taxonomy of the Caribbean excavating sponge species complex Cliona caribaea-C. apricaC. langae (Porifera, Hadromerida, Clionaidae). Caribbean Journal of Science 39(3): 348-370

Zundelevich A, Lazar B, Ilan M (2007) Chemical versus mechanical bioerosion of coral reefs by boring spongeslessons from Pione cf. vastifica. Journal of experimental biology 210(1): 91-96

Zuur A, Ieno EN, Smith GM (2007) Analysing ecological data. Series: Statistics for Biology and Health. Springer Science \& Business Media, New York 


\section{Contributing authors}

Erik H Meesters - Wageningen Marine Research, Wageningen University \& Research, Den Helder, The Netherlands

Fleur C van Duyl - Department of Marine Microbiology and Biogeochemistry, NIOZ Royal Netherlands Institute for Sea Research \& Utrecht University, Texel, Netherlands

Alice $\boldsymbol{E} \boldsymbol{W} \boldsymbol{e b} \boldsymbol{b}$ - Department of Ocean Systems, NIOZ Royal Netherlands Institute for Sea Research \& Utrecht University, Texel, Netherlands

Rolf RPM Bak - Department of Ocean Systems, NIOZ Royal Netherlands Institute for Sea Research \& Utrecht University, Texel, Netherlands

Gerard Nieuwland - Department of Ocean Systems, NIOZ Royal Netherlands Institute for Sea Research \& Utrecht University, Texel, Netherlands

Maggy M Nugues - USR 3278 CRIOBE CNRS-EPHE, CBETM de l'Universite' de Perpignan, Perpignan, France

Steven MAC van Heuven - Department of Ocean Systems, NIOZ Royal Netherlands Institute for Sea Research \& Utrecht University, Texel, Netherlands

Lisanne A van den Bogaart - Marine Biology Research Group, Ghent University, Ghent, Belgium

Evert de Froe - Department of Ocean Systems, NIOZ Royal Netherlands Institute for Sea Research \& Utrecht University, Texel, Netherlands

Estefania Velilla - Department of Ecological Sciences, Vrije Universiteit Amsterdam, Amsterdam, Netherlands

Sophie M Nietzel - Wageningen Marine Research, Wageningen University \& Research, IJmuiden, The Netherlands

Fee Zanke - Wageningen Marine Research, Wageningen University \& Research, Den Helder, The Netherlands

Sil Piek - Rijksuniversiteit Groningen, Groningen, The Netherlands

Yun JH Scholten - Wageningen Marine Research, Wageningen University \& Research, Den Helder, The Netherlands

Sarah SJ Veillat - Wageningen Marine Research, Wageningen University \& Research, Den Helder, The Netherlands

Roger Meijs - Wageningen Marine Research, Wageningen University \& Research, Den Helder, The Netherlands 


\section{English Summary}

Tropical coral reefs are among the most species-rich and productive ecosystems on earth. They cover only $0.1-0.5 \%$ of the ocean floor, but provide a home to almost a third of all fish species and other marine biota. Just like with tropical rainforests, the terrestrial counterpart of these reefs, 3-dimensional complexity underlies this unique biodiversity. On coral reefs, such structural complexity is mainly provided by scleractinian corals. In the interest of growth and protection, these organisms deposit large amounts of limestone, which provides the rigid foundation for the reef. In this way, corals facilitate complex ecosystem functioning and offer shelter and substratum to an exceptional diversity of species. In addition, millions of people depend on these coral reefs, for goods and services such as nourishment (fishing), coastal protection, tourism and recreation. In spite of this, coral reefs are at present under substantial pressure as a consequence of increased human activities. Large-scale global processes such as climate change have a severe impact on these delicate systems, while at the same time a variety of stressors including overfishing, coastal development and pollution substantially reduce reef resilience on a local scale. In this thesis I describe various aspects of the ecological degradation that coral reefs in the Caribbean have undergone since the early 1970s, as a result of factors associated with continuous human population growth (e.g. pollution, overfishing, climate change). The focus of the presented work lies on the coral reefs surrounding Curaçao and Bonaire (southern Caribbean).

\section{Chapter I}

This chapter serves as an introduction explaining the importance of coral reefs and focusses on the main threats that these delicate ecosystems face today. Specifically, it emphases the history and current status of coral reefs in the wider Caribbean region. The main research topics of this thesis are presented, which revolve around the spatio-temporal dynamics of benthic sessile reef communities and the impact that reef degradation and shifts in community composition have on the carbonate budget of the studied reefs.

\section{Chapter II}

Chapter II describes changes in the composition of benthic sessile coral communities on the basis of a 40-year time series. In 1973, Prof. dr. Rolf Bak photographed 16 sections of coral reef on the reef slopes of Curaçao and Bonaire, each measuring $9 \mathrm{~m}^{2}$. Since then, these sections were photographed almost annually. To date, this is the longest running time series on coral reefs in the world, dating from before the mass-mortality of the branched Acropora corals and the Diadema antillarum sea urchins in the 1980s and the major bleaching events in 1998, 2005 and 2010. Quadrats were positioned at four locations along a depth gradient at 10, 20, 30 and 40 meters, respectively. Where the pre-1980 reef configurations are still characterized by exceptionally high cover of scleractinian corals we subsequently see a shift towards a dominance of fleshy macroalgae and algal turf assemblages. These fast-growing opportunistic organisms appear to thrive in the altered reef environment. Reduced herbivory and increased nutrient loads have rapidly provided these algae a competitive advantage over the already weakened corals and calcifying algae. The observed gradual decline in coral to an average cover of less than $10 \%$ 
inherently means a substantial loss of the fundament that underpins the striking biodiversity on these reefs. The rigidity provided by corals can by no means be accommodated by soft-bodied algae. Notably, this chapter describes the manifestation of a novel player that, since the early 2000s, is gaining a foothold on these reefs: benthic cyanobacterial mats. These thick red-brown bacterial assemblages are dominated by cyanobacteria and have become one of the prominent components on these reef. They are comparable to the toxic planktonic cyanobacteria that bloom in freshwater lakes during summertime. Similar to these assemblages, cyanobacterial mats on reefs are known to have a wide variety of negative effects on reef communities including the inhibition of larval settlement, acting as pathogens, smothering other organisms, and creating an anoxic environment. A striking conclusion of this chapter is that the observed path of reef degradation occurs at least down to $40 \mathrm{~m}$ depth. Considering the ongoing increase of anthropogenic impact on the coral reef environment, the rise of cyanobacterial mats in combination with algae and sponges is hypothesized to represent the next alarming step in the degradation of coral reefs.

\section{Chapter III}

In this chapter, shifts in the composition of coral species assemblages are described on the basis of the same time series as presented in the previous chapter. Whereas Chapter II described the trajectories of change of the various sessile organisms, this chapter focusses specifically on the different types of scleractinian corals. Although virtually all coral species declined in cover and abundance over the course of the studied period, a community shift was observed towards dominance of opportunistic species. While the historically prominent framework-building species of the Orbicella and Acropora genera have largely been diminished in the quadrats, species such as Madracis mirabilis, Porites astreoides, Pseudodiploria strigosa (formerly Diploria strigosa), and Agaricia lamarcki appear to possess life-history traits that allow them to better cope with the increasingly more hostile marine environment. Their persistence might make these opportunistic species modest 'winners' in the present-day coral assemblage. Yet, their overall cover declined over the past decades as well. A major concern is that these 'weedy' corals cannot equal the calcification rates of Orbicella and Acropora species, neither can they produce comparable architectural relief. Indeed, the gross calcium carbonate production of these reefs decline, on average, by $67 \%$ over the studied time period.

\section{Chapter IV}

On a healthy reef there is a subtle balance between calcification and erosion, which results in the deposition of calcium carbonate and ultimately net reef accretion. Current global and local disturbance, however, is affecting this balance at increasingly more sites, often pushing it towards net destruction of the limestone structures that form the foundation of these reefs. In addition to a general decline in the abundance of carbonate producing organisms (corals, calcifying algae) (Chapter II and Chapter III), we also see that various bioeroding organisms appear to thrive under the current reef conditions. On many reefs in the Caribbean, excavating sponges are the dominant organisms to bore into the coral skeleton. They do this by chemically dissolving limestone in combination with the mechanical removal of limestone fragments (chips). These sponges are found to increase in both abundance and eroding capacity as a result 
of pollution, ocean acidification and surface water heating. Nonetheless, to date relatively little is known about these organisms. In this chapter various methods, including a flow-through incubation method are presented to quantify erosion rates of the six most prominent bioeroding sponges existing on the reefs of Curaçao. Markedly, the flow-through incubation methodology prevented the accumulation of waste and the depletion of oxygen, resulting in more accurate estimates for chemical erosion. Considerable interspecific variation was observed in the capacity of the various sponges to mechanically remove $\mathrm{CaCO}_{3}$ chips and chemically dissolve coral substrate. This observed interspecific variability could evidently be related to specific lifehistory traits such as the adopted boring strategy (i.e. gallery-forming, cavity-forming or network-working) and presence or absence of symbiotic zooxanthellae. Similarly, a distinct diurnal pattern was observed, where species that have high zooxanthellae densities have higher erosion rates at daytime. Such a pattern was absent in species with a low abundance or complete absence of zooxanthellae.

\section{Chapter V}

Chapter $\mathbf{V}$ describes the effect that decreasing calcification and increasing erosion have on the net production of calcium carbonate on the reefs of Bonaire. Net production and maximum reef accretion potential were determined on the basis of the ReefBudget approach for 115 locations on the shallow reef on the leeward side of Bonaire and Klein Bonaire. The balance is drawn up between the gross biogenic calcification and the gross biogenic erosion, which ultimately yields an estimate for the net carbonate production. For each site, the ReefBudget method was applied to the terrace zone ( $\sim 5 \mathrm{~m}$ depth) and to the shallowest area of the reef slope ( $10 \mathrm{~m}$ depth). The striking results indicate that many shallow reef sites display marginal growth or even net loss of carbonate substrate. The maximum reef accretion potential of the vast majority of the studied reef sites will, in their current configurations, not match the most optimistic projections for of future sea level rise (IPCC 2018: RCP2.6 scenario). Alarmingly, reefs with the lowest vertical accretion potential are found in regions characterized by a low coastline (often $<1 \mathrm{~m}$ above sea level), and include the coastal areas with highest human activity and development around Kralendijk (capital). The current absence of well-developed, rapidly accreting shallow reefs that would otherwise aid in reducing wave energy at the shoreline makes these regions more susceptible to inundation. The risk of flooding will be aggravated especially by the increased frequency and intensity of tropical storms, hurricanes and extreme swell events.

\section{Chapter VI}

In Chapter VI, the new time series described in the previous chapter is used to address both the recent developments within the coral and fish community and the current status of the coral reefs and on the leeward side of Bonaire at remarkably high spatial resolution. A novel classification of present-day benthic reef habitats is proposed for reefs in the lower-terrace and drop-off zones. Four distinct benthic communities are classified within each zone, characterized by distinct configurations of both biotic and abiotic (e.g. sand, rubble) constituents. The majority of the eight newly described communities show little resemblance to pre-1980 configurations. Similarly, a thorough description of reef fish communities is provided for the two zones. Within fish communities, the focus lies on the current population status of herbivorous (parrotfish, 
surgeonfish and damselfish) and commercially important fish species (groupers and snappers). Their abundance and total biomass declined ubiquitously across the shallow reef of Bonaire. Both Chapters V and Chapter VI demonstrate that spatially a distinct gradient of ecological degradation exists on a fine spatial scale (the leeward fringing reef of Bonaire), from nearly pristine reefs in the north to reefs that are at the tipping point of full functional collapse (in front of Kralendijk). The data presented in these chapters further emphasize the negative anthropogenic impacts that locally threaten ecological functioning of coral reefs, but also hints towards stabilization of degradation and possible recovery when local conditions are favourable. The currently existing near-pristine reefs and the reefs to which the latter findings apply should be the ones we learn from to implement adequate management of local stressors.

\section{Chapter VII}

In the final chapter the overall gloomy findings of this thesis are discussed thoroughly. The chapter ends with a positive note, namely that the coral reefs are not lost yet. Mainly the findings described in Chapter V and Chapter VI give a modest reason for hope, at least for the shallower reef zones. The presented work shows that nowadays, under the harsh global environment, it is still possible to maintain reefs that exist in relatively good conditions. The latter appears to be strongly correlated to favourable local conditions (both natural and anthropogenic) (see also Chapter II). These reefs should be studied in detail to reveal the processes underlying their notable ecological success. Furthermore, it is tentatively hypothesized that adequate local management at least in part, facilitated an apparent stabilizing trend observed on the shallow coral reefs around Bonaire. The observation that some of the least degraded reefs are found in marine reserves, where entrance for humans is prohibited, suggests that proper local nature policies indeed aid in making reefs more resilient to the large-scale global disturbance. The main message presented here is that Caribbean coral reefs have been heavily impacted over the past decades but that these reefs and the essential services they provide are not yet lost. Nonetheless, we will have to act now and intervene, on a global and local scale, in order to maintain relatively healthy reefs and improve the condition of severely impacted reefs! 


\section{Nederlandstalige Samenvatting}

Tropische koraalriffen zijn een van de meest soortenrijke en productieve ecosystemen op aarde. Ze bedekken slechts $0.1-0.5 \%$ van de oceaanbodem, maar huisvesten wel bijna een derde van alle vissoorten en andere biota. Net als bij de tropische regenwouden, de terrestrische tegenhanger van deze riffen, ligt de 3-dimensionale complexiteit ten grondslag aan de unieke biodiversiteit. Op koraalriffen wordt deze structuur hoofdzakelijk geboden door koralen. In het belang van groei en bescherming zetten deze organismen grote hoeveelheden kalksteen af, wat fundament geeft aan het koraalrif. Hiermee faciliteren koralen complexe ecosysteem functies en bieden ze een huis aan een uitzonderlijke diversiteit aan soorten. Daarnaast zijn miljoenen mensen afhankelijk van deze koraalriffen, zoals voor voeding (visserij), kustbescherming, toerisme en recreatie. Desondanks staan koraalriffen onder druk door toenemende activiteiten van de mens. Grootschalige globale processen zoals klimaatsverandering hebben een enorme impact op deze systemen, en daarnaast verzwakken ook allerlei lokale stressoren het rif, zoals overbevissing, kustontwikkeling, vervuiling, erosie, en door de mens geïntroduceerde gebiedsvreemde soorten. In dit proefschrift beschrijf ik de ecologische afbraak die koraalriffen in het Caribisch gebied sinds begin jaren 70 van de vorige eeuw hebben ondergaan als gevolg van de impact van voortdurende bevolkingsgroei. De focus van het gepresenteerde werk ligt op de achteruitgang van de koraalriffen gelegen rond Curaçao en Bonaire.

\section{Hoofdstuk I}

Dit hoofdstuk dient als een inleiding waarin het belang van koraalriffen wordt uitgelegd en de bedreigingen voor deze ecosystemen worden geïntroduceerd. Specifiek wordt hier ingezoomd op de historie en de huidige status van de koraalriffen in het Caribisch gebied. De belangrijkste onderzoeksthema's van dit proefschrift worden hier ook gepresenteerd. Deze gaan over de spatio-temporele dynamiek van benthische rifgemeenschappen en de impact die rifafbraak en verschuivingen in gemeenschapssamenstelling hebben op het calcium carbonaatbudget van de bestudeerde riffen.

\section{Hoofdstuk II}

In Hoofdstuk II wordt de verandering in soortensamenstelling van koraalgemeenschappen beschreven aan de hand van een tijdreeks van 40 jaar. In 1973 heeft Prof. Dr. Rolf Bak 16 stukken rif van drie bij drie meter afgemeten op het rifhelling van Curaçao en Bonaire, die vervolgens bijna jaarlijks zijn gefotografeerd. Hiermee is dit de langstlopende tijdserie ter wereld van onderzoek op koraalriffen, daterend van vóór de massale sterfte van de vertakte Acropora koralen en Diadema zee-egels. Op vier locaties zijn kwadranten gepositioneerd langs een diepte gradiënt op respectievelijk 10, 20, 30 en 40 meter. Wat na analyse van de foto's direct opvalt is dat sinds eind jaren 70 gemiddeld $75 \%$ van het levende koraal is verdwenen. Hiervoor in de plaats zijn zachte macro- en turf algen gekomen, snelgroeiende opportunistische organismen die profiteren van de veranderende omstandigheden op het rif. Deze ontwikkeling, ook wel de koraal-algen verschuiving genoemd, heeft het aangezicht van deze riffen aanzienlijk veranderd. Algen kunnen geenszins de fundamentele rol van koralen overnemen, met een aanmerkelijk verlies van de essentiële systeemfuncties tot gevolg. In dit hoofdstuk wordt ook 
een nieuwe speler beschreven die sinds enkele jaren een steeds dominantere positie inneemt binnen de koraalgemeenschap, namelijk de cyanobacterie, die zich presenteert in dikke roodbruine matten op de bodem. Deze complexe gemeenschappen van bacteriën zijn in zekere zin te vergelijken met blauwalgen in zoetwater. Beiden hebben negatieve effecten op het ecosysteem. Cyanobacterie matten verhinderen het vestigen van koraal larven, het zijn ziektekiemen, ze overgroeien andere organismen en creëren 's nachts een zuurstofloze omgeving. Hoogstwaarschijnlijk zijn deze thermotolerante organismen nog efficiënter in het opnemen van nutriënten dan algen en profiteren ze dus van eutrofiëring. Gezien de almaar toenemende invloed van de mens is het niet ondenkbaar dat uitbreiding van deze cyanobacterie matten, in combinatie met algen en sponzen, de volgende alarmerende stap in de afbraak van koraalriffen kan betekenen.

\section{Hoofdstuk III}

In dit hoofdstuk worden, aan de hand van dezelfde tijdreeks als beschreven in het vorige hoofdstuk, verschuivingen binnen de samenstelling van koraalsoorten beschreven. Waar het in Hoofdstuk II voornamelijk ging over de diverse organismen, gaat het hier specifiek over de verschillende soorten kalkskeletvormende koralen. Hoewel nagenoeg alle soorten tijdens de bestudeerde periode gemiddeld in bedekking zijn afgenomen, wordt daarnaast een verschuiving waargenomen van grote rif-bouwende soorten naar kleinere, fenotypisch plastische soorten. Waar de historisch dominante koralen zoals Orbicella spp. en Acropora spp. op veel riffen bijna compleet zijn verdwenen, lijkt het erop dat soorten zoals Madracis mirabilis, Porites astreoides, Pseudodiploria strigosa (formerly Diploria strigosa), and Agaricia lamarcki karaktereigenschappen bezitten waardoor ze beter bestand zijn tegen de steeds vijandiger condities op het rif. De volharding van deze soorten maakt ze op dit moment 'gematigde winnaars' binnen de koraalgemeenschap, waarbij toch ook voor deze soorten geldt dat hun bedekking de laatste decennia is afgenomen. Tevens is de huidige overleving van deze soorten nauwelijks een garantie voor toekomstig voortbestaan wanneer de condities op het rif blijven verslechteren. Een grote zorg is dat deze 'onkruidachtige' koralen niet dezelfde calcificatie snelheid hebben als de Orbicella en Acropora soorten en zij kunnen evenmin de architecturale complexiteit van deze soorten evenaren. De bruto productie van calciumcarbonaat door koralen is over de bestudeerde periode gemiddeld al met $67 \%$ afgenomen.

\section{Hoofdstuk IV}

Op een gezond rif bestaat een balans tussen calcificatie en erosie met als gevolg kalkafzetting en uiteindelijk netto rifgroei. De huidige ontwikkelingen verstoren deze balans op steeds meer riffen en duwen dit evenwicht richting destructie van de kalkstructuren die het fundament vormen van het rif. Naast de afname van kalkproducerende organismen (koralen, calcificerende algen), zien we onder de huidige omstandigheden ook dat bioeroderende soorten beter gedijen. Op veel riffen in de Cariben zijn boorsponzen de dominante organismen die zich in het koraalskelet vestigen. Zij doen dit door kalk actief chemisch op te lossen en door brokjes koraal mechanisch te verwijderen. Deze sponzen nemen zowel in aantal als in erosie capaciteit toe als gevolg van vervuiling, verzuring en opwarming van het oppervlaktewater. Desondanks is nog relatief weinig bekend over deze organismen. In dit hoofdstuk wordt een doorstroom incubatie 
methode gepresenteerd waarmee de erosiesnelheden van zes algemene bioeroderende sponzen op de riffen van Curaçao is bepaald. Deze methode brengt aanzienlijke verschillen in boorsnelheden tussen de soorten aan het licht. De waargenomen variatie, ook tussen dag en nacht, is duidelijk te koppelen aan uiteenlopende levenseigenschappen, zoals de erosie strategie (kamervormend, tunnelvormend, of netwerkvormend) en de aanwezigheid of afwezigheid van endosymbiotische zooxanthellae.

\section{Hoofdstuk V}

In dit hoofdstuk wordt het effect beschreven van afnemende calcificatie en toenemende erosie op de nettoproductie van calciumcarbonaat op de riffen van Bonaire. De nettoproductie is bepaald aan de hand van de ReefBudget methode, voor 115 locaties op het rif aan de lijzijde van Bonaire. Hierbij wordt de balans opgemaakt tussen de bruto biogene calcificatie en de bruto biogene erosie hetgeen uiteindelijk een schatting oplevert voor de netto kalk productie. Per site is de ReefBudget methode toegepast op de terras zone ( $\sim \mathrm{m}$ diepte) en op de meest ondiepe zone van de rifhelling. De verontrustende conclusie van dit hoofdstuk is dat de meerderheid van de sites op dit moment een negatieve kalkproductie heeft. Met name in de terraszone, een zone die voorheen gedomineerd werd door bossen van snel calcificerende Acropora cervicornis koralen, vindt momenteel vrijwel geen rifgroei meer plaats. De riffen in deze ondiepe zone worden het meest direct blootgesteld aan de negatieve effecten van de kustontwikkeling. Het gevolg van deze ontwikkeling is dat het overgrote deel van de riffen rond Bonaire de verwachte zeespiegelstijging niet meer zal kunnen bijhouden en zal Bonaire zijn natuurlijke kustbescherming verliezen. Gezonde riffen met een complexe 3D-structuur breken de golven en verminderen de golfenergie waardoor de kans op overstroming en erosie van de kustlijn aanzienlijk wordt verminderd. De consequenties hiervan zijn al zichtbaar in de vorm van de regelmatige overstromingen van de laaggelegen kustgebieden, inclusief de hoofdstad Kralendijk. De data die in dit hoofdstuk wordt geïntroduceerd zijn de eerste resultaten van een tijdreeks die is gestart in 2014. Deze nieuwe serie is gestart om een ruimtelijke component toe te voegen aan de bestaande foto tijdreeks (Hoofdstuk II en III) die vooral temporele waarde heeft.

\section{Hoofdstuk VI}

In Hoofdstuk VI wordt de in het vorige hoofdstuk beschreven nieuwe tijdreeks gebruikt om zowel de recente ontwikkelingen binnen de koraalgemeenschap als de huidige status van de koraalriffen aan de lijzijde van Bonaire op grote ruimtelijke schaal te beschrijven. Binnen dit hoofdstuk wordt ook een beschrijving gegeven van de typische bentische en vis gemeenschappen die op het huidige rif ontwikkeld zijn. Koralen zijn op veel locaties niet meer de dominante bodembedekkers en andere biotische en abiotische groepen, zoals algen, cyanobacteriële matten, sponzen, zand en koraalpuin karakteriseren de verschillende hedendaagse gemeenschappen. Een van de voornaamste bevindingen binnen dit hoofdstuk is dat enorme variatie bestaat in de mate van rifdegradatie welke afhankelijk is van de lokale invloed van de mens. De riffen gelegen voor de dichtbevolkte hoofdstad Kralendijk worden hedendaags gekenmerkt door een vrijwel volledige functionele instorting van de koraalrif habitat. Anderzijds hebben in gebieden waar de menselijke invloed margináál is de koraalriffen 
nog veel weg van configuraties zoals gezien in de jaren 70 en daarvoor. Deze bevinding biedt hoop voor de toekomst van de koraalriffen, want dit betekent dat onder de huidige globale ontwikkelingen het wel degelijk mogelijk is om gezonde riffen te hebben, mits de lokale omstandigheden hiervoor bevorderlijk zijn. Daarnaast wijzen de bevindingen uit deze nieuwe tijdreeks erop dat de afbraak op delen van het rif de laatste jaren enigszins lijkt te zijn gestabiliseerd op 5 en $10 \mathrm{~m}$ diepte.

\section{Hoofdstuk VII}

In het laatste hoofdstuk wordt ingegaan op de belangrijkste conclusies van dit proefschrift. Daarnaast eindigt dit hoofdstuk met een positieve noot, namelijk dat de koraalriffen nog niet verloren zijn. Voornamelijk de bevindingen beschreven in Hoofdstuk V en VI geven reden tot hoop, in ieder geval voor de ondiepere riffen. Enkele van de minst gedegradeerde riffen zijn gevonden binnen reservaten waar entree voor de mens verboden is. Dat de afname van ondiepe koraalriffen rond Bonaire enigszins lijkt te stabiliseren, zou mede door lokaal ingrijpen gefaciliteerd kunnen zijn. Deze gevolgtrekking duidt erop dat lokaal natuurbeleid deze riffen weerbaarder kan maken tegen de globale invloed van processen zoals de klimaatverandering. De belangrijkste boodschap is wel dat we de koraalriffen en de door deze unieke ecosystemen geleverde diensten alleen kunnen behouden als we per direct zowel lokaal als globaal gaan ingrijpen! 


\section{Acknowledgement}

The acknowledgements that I wrote below sounded way better in my head. Not unlike Polar beers, only tasting good under the sun. Thank you, to all the inspiring places and wonderful people along the way.

First of all, I would like to thank my two supervisors for their confidence in taking me on as their $\mathrm{PhD}$ student and their continuous support over the past years. Fleur, you have never hesitated to share your immense knowledge on the Caribbean reef system, for which I am very grateful. This has not only improved my own understanding of these ecosystems significantly, but has also been extremely helpful in focussing my research objectives and designing relevant research questions. Erik, your ecological view in combination with your statistical expertise has greatly aided me throughout this project. I am specifically appreciative of the PQ-fieldtrips to Curaçao and Bonaire during which we have had the opportunity to discuss, in depth, all aspects of the reef that we encountered on our dives. I look back with great enjoyment to the meetings the three of us had at the NIOZ where we rapidly discussed the relevant issues in about 15 minutes, after which we continued talking about much more humorous subjects for hours.

Han, thank you for guiding me through the process of becoming a Doctor, from the first instant (my job interview) to the last (my public defence). You have allowed me to take the next step in my scientific career and for that I am grateful.

I am privileged to have had such an excellent reading committee. Lisa, already since 2013 you have been an important part of my scientific as well as social life, thank you for our inspiring discussions and your continuous support on so many levels. I sincerely hope that we continue to work together in the future. Mark, over the past years I have seen CARMABI grow under your influence. I have always enjoyed conducting fieldwork there and I am thankful for the way you have facilitated my work without any problems. Katja, when Fleur suggested you as a member of my committee I did not hesitate for a second. Thank you for reading and assessing this work. René, although we have never discussed work on a personal basis, I follow the developments around the algae park on Bonaire with great interest and applaud the initiative. I greatly appreciate that you have taken the time to evaluate my thesis.

I would like to extend a special thanks to Rolf and Gerard. Your work on the permanent quadrats and expertise have given me a jumpstart into my PhD project. I feel privileged that I have had the opportunity to work with both of you. In line with this, I also want to thank Maggy.

I owe both my paranimfen more than I could put down on paper. Olga, just like you chose to have your dad next to you during your defence, I choose to have you, my mother, by my side during this important moment. You have been there for me without exception throughout my entire PhD and I think no paranimf before you has taken the job ever so seriously. Lieve mama, bedankt. Alice, no one has been more closely involved in my $\mathrm{PhD}$ as you have. You are an amazing colleague, a slightly misplaced philosopher, an R genius, a mysterious sea wreck, an 
underrated rapper, Goby Girl superhero, the only real functional alcoholic, and, although you will never agree, an incredibly good scientist. But most of all, you have been an amazing and loyal friend. You have made all my fieldwork trips into unforgettable fun memories and I would not be where I am now if it was not for you. If it were up to me, every $\mathrm{PhD}$ would get a Webb sidekick to make their lives so much more enjoyable. Thank you for everything, I cannot think of anyone else I would want beside me on the $17^{\text {th }}$ of April. It's all good!

The work presented in this thesis would not have been possible without the help of the highly motivated students Joost, Lisanne, Evert, Estefania, Fee, Sophie, Sil, Yun and Sarah.

The majority of my PhD I spent at IMARES/WMR in Den Helder and the NIOZ on Texel. I am grateful for all the great times I have had here and would like to thank all my colleagues at both institutes. In particular, I want to thank Tristan, Andreas, Esmee, Inge, Wim, Irene, Clara, Lennart, Sharyn, Jort, Karel, Frank, Andy, Bob, Judith and Pieternella at the NIOZ and Oscar, Matthijs, Marin, Jimmy, Geert, Dolfi, Ingrid, Diana, Alma, Sophie, Jenny, Michaela, Mardik, Andre, Hans, Joop, Fokje, Steve, Marlies, Willem and Jakob at WMR for making me feel at home here and there, and for all the enjoyable moments and collaboration. Specifically, I would like to express my gratitude to mousquetaire Julie for being an unbelievably kind and wonderful friend and for completing the triangle, as well as Steven for teaching me so much about carbonate chemistry and the great times we had in the lab, on Statia, and in the container on the Pelagia, Tsunami!

I have lived my most memorable moments during my numerous fieldtrips, conferences and the sponge course. That is why I want to acknowledge everyone that I have met in all the wonderful places. Especially, Ben, Valerie, Jasper, Kelly, Kristen, Aschwin, Michelle, René, Friso, Nick, Jeroen, Thomas, Martijn, Robin, Mischa, Fee, Maarten, Bert, Ryan, Roy, Megan, Amanda, Super diver, Franck, Miriam, Tobia, Ayumi, Caren, Wijnand, Johan and Shirley for all the laughs, inspiring discussions, Polars and other intoxicating drinks and everything that makes me look back with nothing but a big smile. Of all these people that have become my friends over the years I need to give it up for my main man Lucas. Thank you for all the great times we had on Curaçao, de Pelagia and in Den Helder and... eine Frage hätte ich noch an dich!

Going through all the steps that precede this moment of finalization is impossible without the support of exceptional friends. That is why I dedicate this section to all my friends whom I have not yet showed my appreciation in these Acknowledgements. Erwin en Reineke, I mention you in this section although actually you are basically family to me. You have always been there, especially in my most difficult moments. I hope I can always count on your powers to rationalize everything! Mat, I think by now it is safe to say that you are my best friend. At the risk of sounding lame, thanks for everything man! Michel, as my oldest friend you have never let me down, you rule! Boris, how much we grew and in the process did away with all nonsense. Oscar, thanks for the random moments we could hang out and chill. Elze, thank you for your eternal optimism. Robin, my man in Nijmegen. Thank you for your dry humor. Rock on! Casey, my far-away friend, thanks for preparing me for the $\mathrm{PhD}$ process during our internship together. Jonathan, Laszlo and Ruben, although we do not see each other that often anymore, our 
friendship has meant a lot to me these years. I hope we still manage to go to London any time soon. Daan, I want to thank you and your family for taking me along on your hunting trips to Frieswijk, Limburg and Noord-Holland. The excitement surrounding these trips has given me extra motivation when I was completely done with work. Furthermore, I would like to thank all members of the Egypt trips, the Ajax gang and de Heeren van Gouda for taking my mind off work at those moments where I really needed it.

Hanna, there are not many that have supported me more during the first years of my $\mathrm{PhD}$ than you have. From the moment I heard I got the position you have stood by my side without hesitation. I am indescribably grateful to everything that you, Annemarie, Kees, Simon, Grosi, Grossvati, Marietta and Walter have given me.

Dans cette section, je tiens à remercier deux personnes très spéciales à mes yeux. Jean-Pierre et Marie Jeanne, merci pour tout ce que vous avez fait pour moi au cours de ces 20 dernières années. Vous m'avez traité comme un fils lors de chacune de mes visites a Rochetaillée. JeanPierre, vous m'avez appris tous les secrets des chasseurs renommés de Rochetaillée et pour cela je suis éternellement reconnaissant. Je suis si heureux que vous soyez présent pour ma soutenance de thèse.

Finally, I want to take this opportunity to thank my family. I could not wish for a more supporting, kind, funny and gezellige family than the De Bakkers. All of you have always been there for me, in the good times but also in the bad ones. To have such a wonderful family behind has made it possible to be where I am today.

Lieve opa, oma en Gerhard, het begin van dit proefschrift ligt in Zuid-Frankrijk, op een bergtop vlak aan de rand van Azillanet. Hier, op jullie ruige terrein ontstond mijn passie voor de biologie. Jullie hebben mij altijd gesteund en zijn altijd zo trots. Dat jullie er bij zijn tijdens mijn verdediging maakt mij intens gelukkig. Niemand kan zich fijnere grootouders wensen. Lieve Annie en Gerda, bedankt voor alle fijne en motiverende momenten. Ik ben blij dat jullie er op 17 april bij kunnen zijn.

Pieter, je bent al lang niet meer alleen mijn zwager, je bent een van mijn beste vrienden geworden. De afgelopen jaren hebben we zo veel geweldige momenten gehad en ik weet zeker dat deze momenten van ultiem relaxen essentieel zijn geweest tijdens mijn $\mathrm{PhD}$. Thanks Bro!

Ralf, we kennen elkaar nog niet zo goed, maar ik weet zeker dat dit nog gaat komen. Bedankt voor alle heerlijke maaltijden en gezellig momenten in het afgelopen jaar.

Lieve Madeleine, mijn kleine zusje. Ik kan niemand bedenken die zonder uitzondering zo ontzettend lief is geweest voor mij. Ik kan altijd bij je binnenlopen en rekenen op een gezellige avond met een goed biertje of een uitstekende wijn. Je hebt het niet altijd makkelijk gehad, maar ik ben ontzettend trots op hoe jij je in de laatste jaren hebt ontwikkeld, helemaal als alfa omringd door bèta's. Je kiest altijd je eigen weg en blijft dan ook resoluut achter je keus staan. Daar heb ik nog wel het een en ander van geleerd en kunnen toepassen tijdens mijn promotie. Bedankt dat je zo'n fijn zusje bent. 
Lieve Bernadette, een ding weet ik zeker, zonder jou was ik nooit zo ver gekomen. Vanaf de basisschool heb ik jouw pad kunnen volgen en nooit het wiel opnieuw hoeven uitvinden. Jij staat aan de basis van mijn carrière als mariene bioloog, mijn eerste momenten in het water waren altijd samen met jou. In het zwembad bij opa en oma, tijdens het wedstrijdzwemmen, bij mijn eerste duiken en op onze vakanties samen naar de Turks \& Caicos en Indonesië. Ik kan mij niet voorstellen hoe ik dit allemaal had moeten doorlopen zonder te kunnen rekenen op jouw ervaring en adviezen. In jouw acknowledgements noem je al dat wij twee handen op een buik zijn en niets is minder waar. Mijn lieve zus, bedankt voor alles wat je voor mij hebt gedaan en bedankt voor de geweldige cover van mijn thesis.

Lieve papa en mama, tijdens mijn gehele ontwikkeling heb ik op jullie kunnen rekenen en hebben jullie er altijd alles aangedaan om mij aan te sporen de volgende stap te zetten. Als Azillanet de plaats is waar mijn passie voor biologie ontstond, dan zijn As en Rochetaillée de plekken waar ik bioloog geworden ben. Mijn interesse in de biologie is een directe uitkomst van jullie liefde voor de natuur. Jullie hebben mij altijd gestimuleerd om te ontdekken en het willen ontdekken is de belangrijkste eigenschap van een onderzoeker. Jullie hebben een gezin gecreëerd waar alleen maar liefde in bestaat en dat is iets wat meer dan wat dan ook bij heeft gedragen aan wie ik nu ben. Alles wat ik hier opschrijf doet tekort aan jullie bijdrage in mijn leven. Ik wens iedereen zulke fantastische ouders toe. 


\section{Biography}

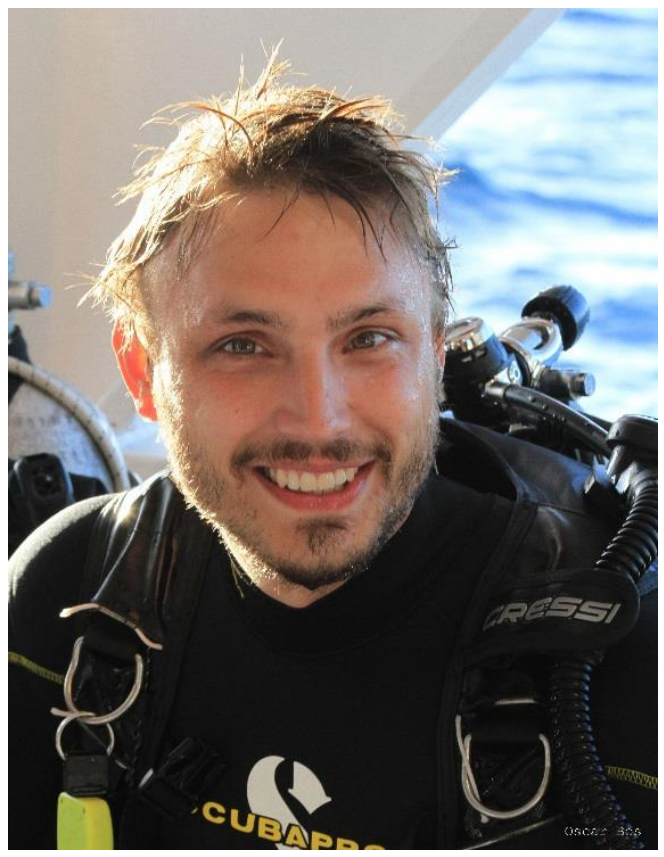

Didier was born on the $10^{\text {th }}$ of November 1988 in Amsterdam and named after his two grandfathers, Desiderius Marinus de Bakker. He is the son of Henri and Olga de Bakker and was the middle child between his two sisters Bernadette and Madeleine. Already at a young age he was fascinated by nature in all its aspects. It was not surprising that as soon as he graduated from the Sint Antonius College in Gouda in 2007, he started the bachelor General Biology at the University of Amsterdam. His passion for water led him to start scuba diving in order to, together with his big sister, experience the underwater world with his own eyes. In the last year of his Bachelor he chose to follow the aquatic track to, subsequently, enrol into the Master Limnology and Oceanography at the same university. During his two Master internships he studied the recycling of dissolved organic matter by sponges on the reefs of Curaçao under the supervision of Dr. Ir. Jasper de Goeij and the population genetic structure of corals and sponges in the Dutch Caribbean under the supervision of Dr. Lisa Becking. After finishing his Master in 2014 he got the opportunity to start a PhD at Wageningen University supervised by Dr. Erik Meesters (Wageningen Marine Research) and Dr. Fleur van Duyl (Royal Netherlands Institute for Sea Research). During his PhD he had the privilege to work on the longest running time series of photo quadrats (positioned on the reef slopes of Curaçao and Bonaire) on coral reefs in the world. This work, in combination with the work he conducted with his colleague $\mathrm{PhD}$ Alice Webb in the Dutch Caribbean forms the basis of this thesis. 


\section{Bibliography}

Bart MC, de Vet SJ, de Bakker DM, Alexander BE, van Oevelen D, van Loon EE, van Loon JJWA, de Goeij JM (2019) Spiculous skeleton formation in the freshwater sponge Ephydatia fluviatilis under hypergravity conditions. PeerJ 6: e6055

Van Heuven SM, Webb AE, De Bakker DM, Meesters EH, Van Duyl FC, Reichart G-J, De Nooijer LJ (2018) In-situ incubation of a coral patch for community-scale assessment of metabolic and chemical processes on a reef slope. PeerJ, 6: e5966

De Bakker DM, Webb AE, van den Bogaart LA, van Heuven SM, Meesters EH, van Duyl, FC (2018) Quantification of chemical and mechanical bioerosion rates of six

Caribbean excavating sponge species found on the coral reefs of Curaçao. PloS One, 13(5): e0197824

Kenny NJ, de Goeij, JM, De Bakker DM, Whalen CG, Berezikov E, Riesgo A (2018)

Towards the identification of ancestrally shared regenerative mechanisms across the Metazoa: A Transcriptomic case study in the Demosponge Halisarca caerulea. Marine genomics, 37: 135-147

Webb AE, van Heuven SM, De Bakker DM, van Duyl FC, Reichart G-J, de Nooijer LJ (2017) Combined effects of experimental acidification and eutrophication on reef sponge bioerosion rates. Frontiers in Marine Science, 4: 311

De Bakker DM, Van Duyl FC, Bak RPM, Nugues MM, Nieuwland G, Meesters EH (2017) 40 Years of benthic community change on the Caribbean reefs of Curaçao and Bonaire: the rise of slimy cyanobacterial mats. Coral Reefs, 36(2): 355-367

De Bakker DM, Meesters EH, Bak RPM, Nieuwland G, Van Duyl FC (2016) Long-term shifts in coral communities on shallow to deep reef slopes of Curaçao and Bonaire: are there any winners? Frontiers in Marine Science, 3: 247

De Bakker DM, Meesters EH, van Bleijswijk JD, Luttikhuizen PC, Breeuwer, HJ, Becking LE (2016) Population genetic structure, abundance, and health status of two dominant benthic species in the Saba Bank National Park, Caribbean Netherlands: Montastraea cavernosa and Xestospongia muta. PloS One, 11(5): e0155969

De Bakker DM, Wilkinson M, Jensen B (2015) Extreme variation in the atrial septation of caecilians (Amphibia: Gymnophiona). Journal of anatomy, 226(1): 1-12. 


\section{Photo specification}

Page 7: Close up of Meandrina meandrites coral

Page 8: Close up of Colpophyllia natans

Page 14: Small sponge (callyspongia plicifera) surrounded by cyanobacterial mats and Dictyota spp. macroalgae

Page 42: Diploria labyrinthiformis coral on an eroded reef

Page 66: Excavating sponge Siphonodictyon brevitubulatum (yellow ostia and oscula) in old coral head

Page 92: Close up of Acropora cervicornis branches at a coral restoration site on Bonaire Page 114: Brittle star on Aplysina archeri sponge

Page 148: Juvenile Diadema antillarum se

Page 156: Caribbean reef squid (Sepioteuthis sepioidea) 


\section{SENSE}

Netherlands Research School for the

Socio-Economic and Natural Sciences of the Environment

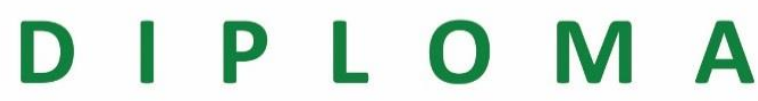

For specialised PhD training

The Netherlands Research School for the

Socio-Economic and Natural Sciences of the Environment

(SENSE) declares that

\section{Didier (Desiderius) Marinus de Bakker}

born on 10 November 1988 in Amsterdam, The Netherlands

has successfully fulfilled all requirements of the Educational Programme of SENSE.

Wageningen, 17 April 2019

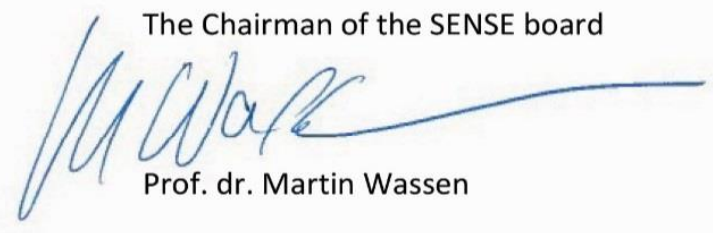

the SENSE Director of Education

The SENSE Research School has been accredited by the Royal Netherlands Academy of Arts and Sciences (KNAW)

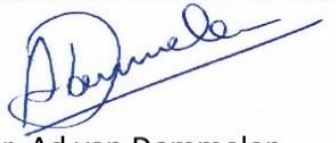

Dr. Ad van Dommelen

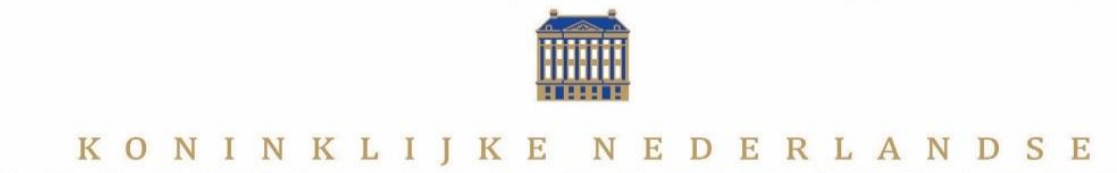

$\begin{array}{llllllllllllllllllllllll}\text { A } & K & A & D & E & M & I & E & V & A & N & \text { W } & E & T & E & N & S & C & H & A & P & P & E & N\end{array}$ 


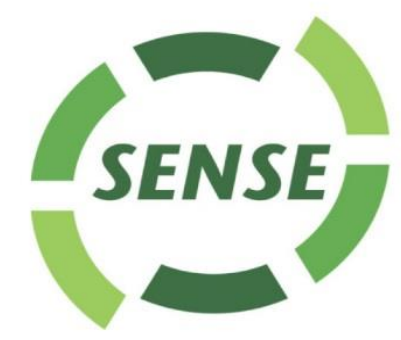

The SENSE Research School declares that Didier (Desiderius) Marinus de Bakker has successfully fulfilled all requirements of the Educational PhD Programme of SENSE with a work load of $34.3 \mathrm{EC}$, including the following activities:

\section{SENSE PhD Courses}

- Environmental research in context (2015)

- Basic statistics (2018)

- Research in context activity: 'Making on online Research video: A PhD in the Caribbean' (2019)

\section{Other PhD and Advanced MSc Courses}

- How to write a scientific paper, Royal NIOZ (2014)

- Giving a scientific talk, Royal NIOZ (2014)

- Acquiring skills in stereo-camera photography and 3D-modelling software, Wageningen Marine Research (2014-2015)

- Acquiring skills in marine carbon chemistry and optical alkalinity titration (photospectrometry), Royal NIOZ (2015-2018)

- NIOZ writing week Royal NIOZ $(2017,2018)$

\section{External training at a foreign research institute}

- Introduction into R for statistical analyses, Highlands Statistics UK (2015)

- Sponge Biodiversity Course, Mote Marine Laboratory, Florida, USA (2015)

- Nature Master Class Online (NMO), Nature, USA (2018-2019)

\section{Management and Didactic Skills Training}

- Supervising MSc student with thesis entitled 'Excavating sponge community composition and habitat preferences on the Caribbean reefs of Curaçao' (2015)

- Supervising MSc student with thesis entitled 'Variations in bioerosion rates of different Caribbean Excavating sponge species' (2017)

- Co-supervision of 11 other BSc and MSc students (2014-2018)

\section{Oral Presentations}

- 40 years of benthic community change on the coral reefs of Curaçao and Bonaire. International Coral Reef Symposium, 19th -24th June 2016, Honolulu, Hawaii

- A new approach to estimate bioerosion rates of six excavating sponge species. World Sponge Conference, 25 - 30 June, 2017, Galway, Ireland

SENSE Coordinator PhD Education

Dr. Peter Vermeulen

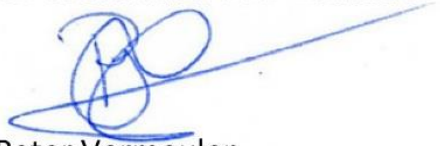


The research described in this thesis was financially supported by Wageningen University, Wageningen Marine Research (WMR), The Royal Netherlands Institute for Sea Research (NIOZ) and with additional funding from the Ministry of Economic Affairs.

Financial support for the publication of this thesis was kindly provided by:
* Hippocampus Medicus, medische (duik)keuringen
* Kantoor van Mil Belastingadviseurs te Nieuwegein, de specialist voor medici
* Dive Post Zoetermeer, the place where diving starts!
* Subtronic, Professionelle Unterwasser Lichttechnik

Cover design and lay-out: B.S. de Bakker \& D.M. de Bakker

Printed by IPSKAMP printing theses and more

C2019 by DM de Bakker

All rights reserved. No parts of this thesis may be reproduced, stored in a retrieval system, or transmitted in any form or by any means without permission of the author or copyright holding journal. 


\section{Propositions}

1. Benthic cyanobacterial mats will become an increasingly more abundant player on coral reefs as coastal water quality continues to deteriorate.

(this thesis)

2. Restoring Caribbean coral reefs to pre-1980 configurations is an illusion as long as humans are walking this earth.

(this thesis)

3. Wind turbines and solar panels spread across the vast plains of the Sahara Desert should already be our main source of renewable energy. (Li et al. 2018, Science)

4. The current system in which scientists have to fight for the same research funds hampers scientific progress.

5. The public 'hunt' on the hunter instigated by so-called nature activists completely misses its target, namely nature conservation.

6. In a society and time without real problems, irrelevant issues become mainstream.

7. The decline in the quality of music since the early 1970s parallels the ecological decline of coral reefs over the same period.

Propositions belonging to the thesis, entitled

\section{0 years of change on the coral reefs of Curaçao and Bonaire}

Didier M. de Bakker

Wageningen, April $17^{\text {th }} 2019$ 


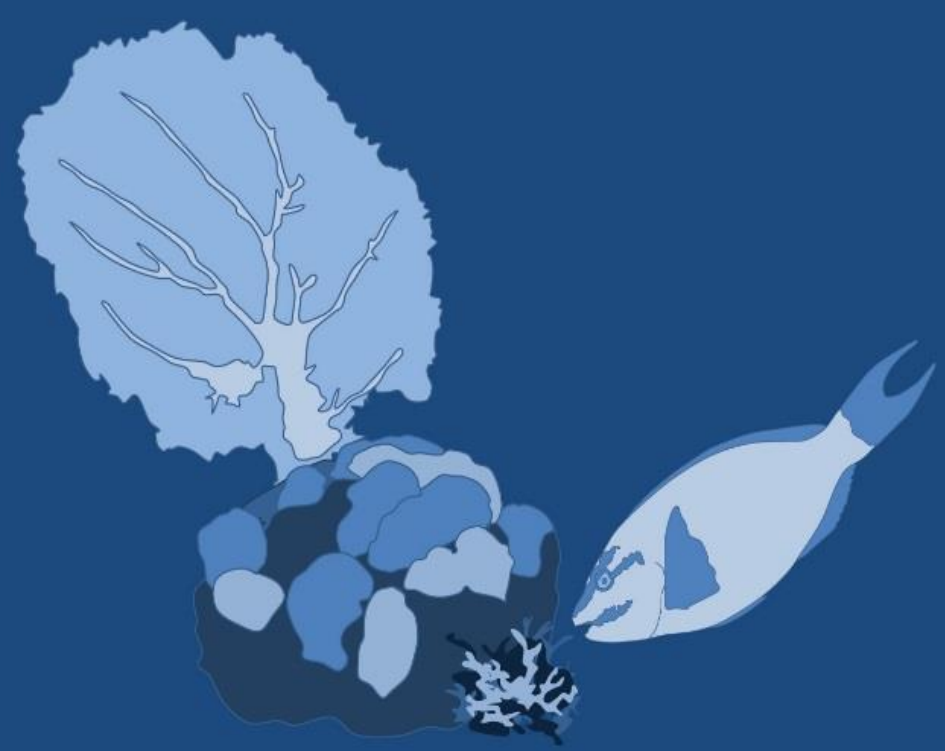

